POLYTECHNIC UNIVERSITY OF VALENCIA

DEPARTMENT OF CHEMISTRY

INSTITUTE OF CHEMICAL TECHNOLOGY (UPV-CSIC)
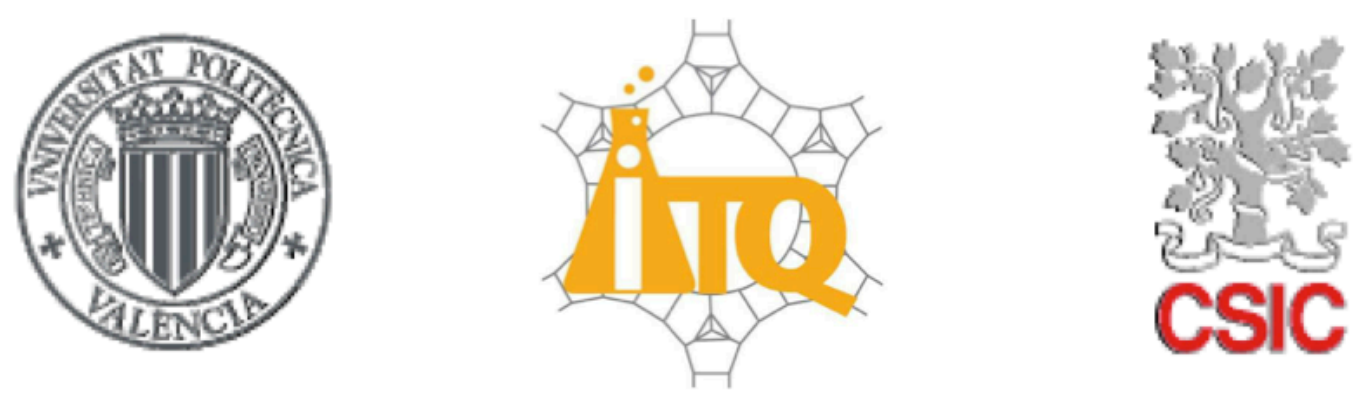

PHOTOCATALYST BASED ON TITANIUM OR IRON SEMICONDUCTORS FOR THE GENERATION OF HYDROGEN FROM WATER UPON SOLAR IRRADIATION

\author{
PhD. Thesis \\ MARCO SERRA \\ SUPERVISOR:
}

Prof. Hermenegildo García Gómez

Dr. Pedro Atienzar Corvillo

Valencia, February 2015 



\section{PHOTOCATALYSTS BASED ON TITANIA OR IRON SEMICONDUCTORS \\ FOR THE GENERATION OF HYDROGEN FROM WATER UPON SOLAR IRRADIATION}

Table of Contents

1 Introduction

1.1 Introduction

1.2 Hydrogen as energy vector 7

1.3 Photocatalysts for hydrogen generation _ _ _ 9

1.4 Modified $\mathrm{TiO}_{2} \ldots 13$

$1.5 \mathrm{Au}$ NPs supported on $\mathrm{TiO}_{2}$ as visible light photocatalyst for hydrogen generation 15

1.6 Heterojunction photocatalysts 18

1.7 Framework phosphate as photocatalysts _ 21

1.8 References _ 25

2 Objectives _ 27

2.1 Objectives _ 29

3 Influence of the irradiation wavelength on the photocatalytic activity $\mathrm{Au}-\mathrm{Pt}$ nanoalloy supported on $\mathrm{TiO}_{2}$ for hydrogen generation from water 31

3.1 Introduction _ 33

3.2 Results and discussion 34

3.2.1 Sample preparation and characterization _ 34

3.2.2 Photocatalytic activity for the $\mathrm{H}_{2}$ generation under visible light irradiation 39

3.2.3 Photocatalytic activity of $\mathrm{Au}-\mathrm{Pt} / \mathrm{TiO}_{2}$ under UV light or simulated sunlight 44

3.2.4 Role of Pt in $\mathrm{Au}-\mathrm{Pt} / \mathrm{TiO}_{2} \ldots 47$

3.3 Conclusions _ 47

3.4 References _ 48 
4 Photocatalytic hydrogen generation from water methanol mixutres using "black" anatase obtained by annealing of titanate nanotubes $\ldots$

4.1 Introduction 53

4.2 Results and discussion $\ldots \ldots$

4.2.1 Hydrogen production 60

4.3 Conclusions

4.4 References 65

5 Synergy of the combination of titanate nanotubes with titania nanoparticles for the photocatalytic hydrogen generation from water methanol mixture using simulated sunlight

5.1 Introduction 69

5.2 Results and discussion

5.3 Conclusions _.... 79

5.4 References _ 80

6 Visible light photocatalytic activity for hydrogen production from water methanol mixtures of open-framework V-doped mixed valence titanium phosphate _ _ 83

6.1 Introduction 85

6.2 Results and discussion 86

6.2.1 Photocatalytic hydrogen generation 95

6.2.2 Characterization of $\left(\mathrm{Ti}^{3+} / \mathrm{Ti}^{4+}\right) \mathrm{P}$ as photocatalyst

6.3 Conclusions

6.4 References 108 
7 Doped framework iron phosphate as novel photocatalyst for hydrogen production from water methanol mixtures

7.1 Introduction

7.2 Results and discussion

7.3 Conclusions

8 Materials and method

8.1 Emission spectra of solar simulator

8.2 Emission spectra of visible light LED

8.3 Influence of the irradiation wavelength on the photocatalytic activity of Au-Pt nanoalloys supported on $\mathrm{TiO}_{2}$ for hydrogen generation from water

8.3.1 Samples preparation 136

8.3.2 Samples characterization $\quad 136$

8.3.3 Photocatalytic tests for hydrogen production $\quad 136$

8.4 Photocatalytic hydrogen generation form water-methanol mixtures using "black" anatase obtained by annealing of titanate nanotubes

8.4.1 Samples preparation 137

8.4.2 Photocatalytic tests for hydrogen production _ 137

8.5 Synergy of the combination of titanate nanotubes with titania nanoparticles for the photocatalytic hydrogen generation from water methanol mixtures using simulated sunlight

8.5.1 Samples preparation 138

8.5.2 Photocatalytic tests for hydrogen production 139

8.6 Visible light photocatalytic activity for hydrogen production from water-methanol mixtures of open-framework V-doped mixed-valence titanium phosphate _.... 139

8.6.1 Material Preparation 139

8.6.2 Photocatalytic tests for hydrogen production 140

8.6.3 Photocurrent measurements and determination of flat band potential of the conduction band $\quad 140$

8.6.4 Transient absorption spectroscopy _ 141 
8.7 Doped framework iron phosphate as novel photocatalyst for hydrogen production from water methanol mixtures $\quad 144$

8.7.1 Material Preparation _ 144

9.7.2 Photocatalytic tests for hydrogen production _ _ 144

8.7.3 Photocurrent measurements and determination of flat band potential of the conduction band 144

8.7.4 Transient absorption spectroscopy 145

8.8 References 146

9 Conclusions 147

9.1 Conclusions _ 149

10 Appendix 151

10.1 Abstract 153

10.2 Publications _ 156 
Chapter 1

Introduction 


\section{Chapter 1 - Introduction.}

\subsection{Introduction.}

Modern societies are based on massive energy consumption. A survey of 2010 on the contribution of different energy resources to the total energy consumption in the USA has shown that about of $80 \%$ of the consumed energy derives, in a way or another, from fossil fuels and coke.

Due to the limited resources of fossil fuels as well as the uneven geographical distribution of oil and natural gas in the planet, there is an increasing concern for developing alternative primary energy resources. Different predictions have forecast that the current capacity for oil and natural gas extraction should decline in the forthcoming years, due to the logical depletion of the natural resources. In addition, due to the emergence of third world countries as world players in economy, predictions also for see an increase in the per capita consumption of energy in the next decade. This increase in energy needs could never been covered based on fossil fuels. All the predictions with regard to the availability of fossil fuels have fortunately became inaccurate due to better exploitation of the oil fields and the new technology to exploit bituminous sands, it is clear that at the current consumption rate of oil and natural gas, these energy resources should become increasingly depleted in a few decades.

Another aspect to be considered besides limited resources is the impact that the combustion of fossil fuels is playing on the Earth climate and Environment pollution. The generation of $\mathrm{CO}_{2}$ and other greenhouse gases has increased steadily the average temperature of the Earth. Reports of the International Panel on Climate Change (IPCC) have predicted that continuing the current scheme of energy consumption cannot be sustained and that the planet is facing to a gradual temperature increase and global warming that should leave according to the model to dramatic change with regard to sea level, intensity of rain and other weather conditions. 
Figure 1.1. Electricity generation by fuel for the period 1990-2040 (trillion kilowatt-hours) issued by U.S. Energy Information Administration in April 2014.

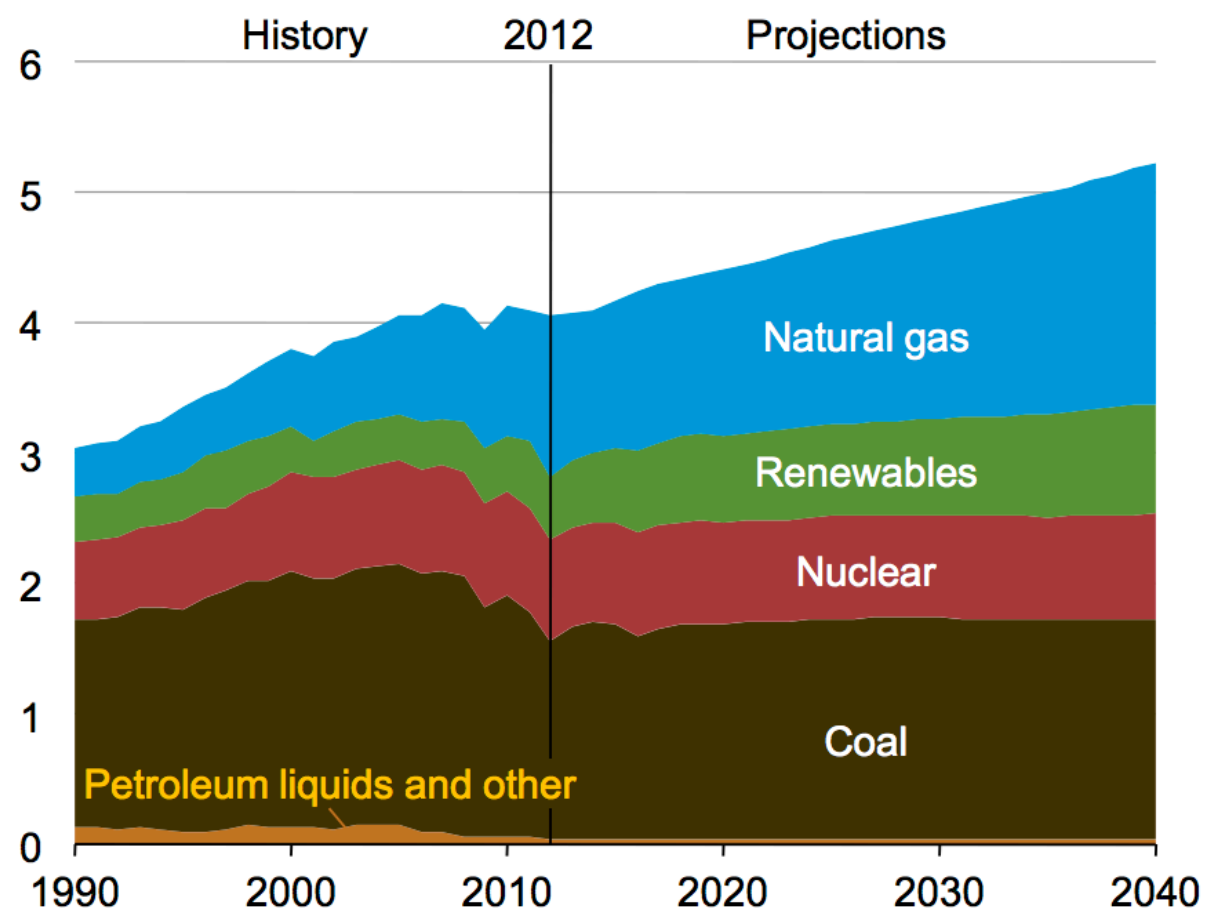

In addition, combustion of fossil fuels has also created severe pollution problems due to the presence in the gases after the combustion of $\mathrm{SO}_{\mathrm{x}}, \mathrm{NO}_{\mathrm{x}}$ and unburned hydrocarbons. Sulphur and nitrogen oxides are responsible for the acid rain that has caused the deforestation of wide wild woods. In addition, the pollutants from the combustion of fossil fuels cause problems in the health of exposed animal and human beings, particularly asthma and other respiratory problems as well as circulatory dysfunctions. In order to decrease the percentage of $\mathrm{SO}_{\mathrm{X}}$ emitted to the atmosphere, and considering that the origin of sulphur is its presence in the fossil fuels, legal regulations in the developed countries about the quality of fuels have been increasingly rigorous on the sulphur content allowed in the fuels. In EU and most Western Countries, fuels for automotive transportation should have the sulphur content of about a few ppms or even in the ppb scale in the USA. To achieve this sulphur concentration, oil refining has implemented increasingly efficient desulfurization processes such a hydrodesulfurization, alkylation of sulphur compounds, softening of fuel stream and more recently oxidative desulfurization treatments. 
The reduction in the content of $\mathrm{NO}_{\mathrm{x}}$ is, however, much more difficult to be achieved since nitrogen element is not present in the fuel, but in the air. The recent EU directive about the quality of air has limited the tolerable $\mathrm{NO}_{\mathrm{x}}$ concentration in urban air to about $20 \mathrm{ppm}$. However, in rash hours under circumstances of heavy traffic, this tolerable $\mathrm{NO}_{\mathrm{x}}$ concentration is overcome by even one order of magnitude.

In addition, the chronic and continuous exposure to NOx, even at lower concentration is highly risky for human health. In this case, however, $\mathrm{NO}_{\mathrm{x}}$ is originated from the reaction of $\mathrm{N}_{2}$ and $\mathrm{O}_{2}$ inside the motor engine, due to the high temperature reached under these conditions and the thermodynamic equilibrium of $\mathrm{N}_{2}$ and $\mathrm{O}_{2}$ mixture.

The alternately to avoid $\mathrm{NO}_{\mathrm{x}}$ in the air include to reduce the temperature of the combustion below $1000{ }^{\circ} \mathrm{C}$ to avoid reaching the thermodynamic equilibrium between $\mathrm{N}_{2}$ and $\mathrm{O}_{2}$ and their oxides, to introduce in the engine exclusive oxygen, rather than air, to perform the combustion or to treat the exhaust gases with catalysts to effect the complete oxidation of $\mathrm{NO}_{\mathrm{x}}$ to nitrate.

Considering the above two aspects, limited resources and climate pollution effect, it is evident that it is of considerable importance to change urgently primary energy resources from fossil to alternative resources that should be abundant and without producing $\mathrm{CO}_{2}$ or other greenhouse gases. Besides geothermal sources of various nature including wind, sea tides and waves, hydraulic energy and so on, Sun is a primary almost inextinguishable primary energy source. It has been calculated that just a few per cents of the sunlight energy should be sufficient to provide the energy required for the Humankind in the future. It is clear that Sun as primary energy resource is behind the life origin in the planet and also has been involved in the formation of fossil fuels.

There are many different possibilities to convert sunlight into other derived energy sources, including solar furnaces that concentrate light beams to a local point in where the temperature is above $1000{ }^{\circ} \mathrm{C}$ and other heating devices (that can be used to heat liquid into mechanical devices that are able to produce electric energy).

Other alternative to use solar light is to convert this energy into electricity. The main two problems of solar light as primary energy resource are the low energy 
density per surface area and the dependence of the light intensity with solar light fluctuations including seasons, day and night cycles and weather conditions. For these reasons, it would be for many applications extremely difficult to use directly sunlight to supply energy. It would be much more convenient to "accumulate" and store the energy of the sun into a secondary energy system. In this regard the conversion of solar light into electricity has to been coupled with an additional storage system that should provide electricity on demand. One classical approach has been the use of electrical accumulator (battery) or the use of supercapacitors. It has been proposed that lithium batteries should be an ideal system to accumulate photovoltaic electricity provided by solar panels for automotive purposes. For transportation one parameter that is of considerable importance is the energy density of the accumulator device, meaning the energy per unit of weight. Since Li is the lightest non gaseous element and, in principle, 3 grams of this element can accumulate up to 96500 Coulombs, lithium ion battery is the lightest possible electrical accumulator. However, one or the main drawbacks of this approach based on the transformation of sunlight into electricity by means of solar panels and lithium batteries is the low relative abundance of lithium in the Earth. In fact, there are only a few geographical location including Argentina (El Salar del Hombre Muerto), USA (Yutah) and Afghanistan are the three main places where lithium can be obtained.

An alternative possibility to store sunlight would be to use sunlight for the production of a chemical compound that could be use later on demand to provide the energy. Since Earth atmosphere contains oxygen as component, most of the chemical compounds that have been proposed in this approach are reduced chemicals.

This has led to a new concept that has been denoted as "Solar Fuels" meaning that the proposal is to obtain chemicals that subsequently can be used as fuels for transportation, but that are obtained by means of solar light conversion into energy. Solar fuels are based on the transformation, typically photocatalytic, by reduction of abundant precursors. Particularly important is the generation of hydrogen from water and photocatalytic $\mathrm{CO}_{2}$ reduction by water. 


\subsection{Hydrogen as energy vector.}

The US department of energy considers that hydrogen is the ideal fuel for transportation, since it could be obtained from water and when reacted with oxygen under ambient conditions using fuels cells, the only by-product of the reaction would be water without generation of $\mathrm{SO}_{\mathrm{x}}$ or $\mathrm{NO}_{\mathrm{x}}$. However, the department of energy has identified different gaps in fundamental science and technology that makes at the presents impossible the implementation of hydrogen as fuels [1]. In addition of pinpointing the gaps in science and technology to implement hydrogen, the US department of energy has also set a serious increasing of milestones to be achieved in order to overcame the current limitations. Specifically the Department of Energy has detected a lack in technology for generation of hydrogen from water that directly o indirectly uses sunlight as primary energy. Other important technology gap refer to hydrogen storage at room temperature and atmospheric pressure and room temperature and hydrogen oxidation using fuels cells that do not contain noble metals. Specifically for the purpose of the present Thesis, the point that would be subject of study would be the development of more efficient sunlight photocatalysts for hydrogen generation from water. In this area, and considering the current high consumption of hydrogen at industrial scale, the target would be to develop an economically viable process for hydrogen generation that could compete with other technologies based on fossil fuels transformation, for which a price of reference would be about $5 €$ per kg of hydrogen.

There are different alternative for hydrogen generation from water based on solar light. The more important are the thermal water splitting, the combinations of photovoltaic cell and water electrolysis and the use of a photocatalyst to convert directly photons into chemical energy as hydrogen. All these three approaches have currently important bottlenecks that severely limit their application at the current state.

Thermal water splitting requires for thermodynamic reasons, high temperatures of about $2000^{\circ} \mathrm{C}{ }^{[2]}$ and this high temperatures can only be achieved in solar furnaces in which many light beams are collected by mirrors and concentrate the sunlight into a tower in where the furnace is located. This technology is highly sensitive to weather conditions and particularly to the presence of clouds and seasonal 
variations. In addition it requires very large areas (solar orchids) in where mirrors having to change the orientation continuously during the day following the Sun trajectory ("heliostats") are located. The investment cost of this technology is the highest, due to the need of heliostats, the large surface area and the construction of a solar tower. The mayor limitations of this technology is the low efficiency of heat storage in melted ionic liquids and the need to handle a large weight of catalyst that effect the thermal water splitting with a low efficiency in terms of mol of hydrogen generated per kg of catalyst. [3] Typically catalyst for this thermal water splitting are not stoichiometric metal oxides such as a iron oxide or other transition metal and lanthanide oxide ${ }^{[4]}$, that are able to react with water gaining oxygen and releasing hydrogen that much higher temperatures evolve oxygen from the inorganic lattice. Scheme 1.1. illustrates the cycle operating in the thermal water splitting based on no stoichiometric metal oxide ${ }^{[4]}$.

Scheme 1.1. Elementary steps involved in the thermal water splitting catalysed by non stoichiometric oxide.

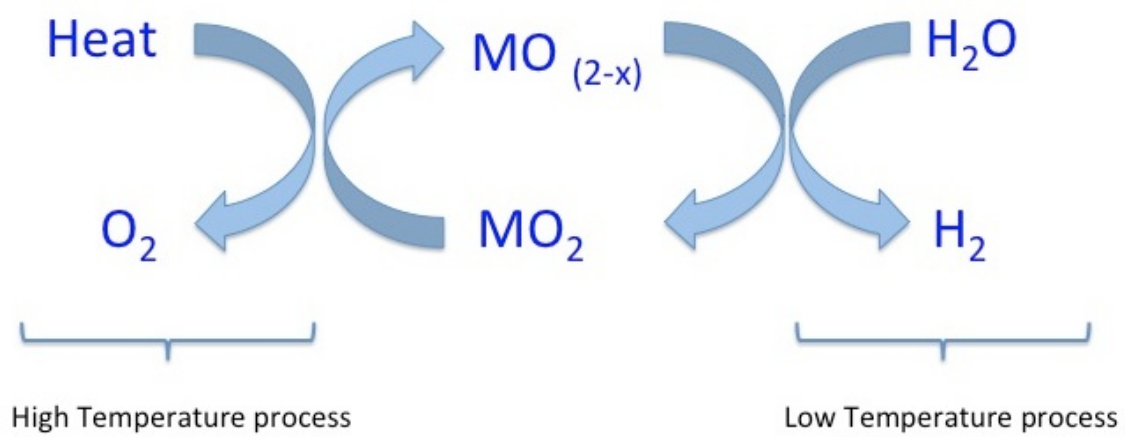

In the two-step process combining photovoltaics and water electrolysis, the main limitations are the efficiency of current photovoltaic cell and the high electrochemical overpotential needed for oxygen generation from water at neutral $\mathrm{pH}$.

Photovoltaic cells have to be combined in series in order to reach the voltage required to overcome this overpotential, but these electrical connections reduce notably the efficiency that can be achieved with individual cells. In addition, the nature of the electrode to produce the electrocatalysis is also another issue to be solved in order to reduce the costs, increase the lifetimes and increase the 
efficiency of the system. In addition the investment cost of photovoltaic cells combined with electrocatalyst is also much higher than desirable and requires adequate maintenance, particularly to avoid passivation or corrosion of electrodes.

\subsection{Photocatalysis for hydrogen generation.}

For all the above reasons, the use of photocatalysis based on the use of a powder dispersed in water that could directly generate hydrogen from sunlight is an appealing technology that may require much lower investments and can easily adapted to small plants to supply domestic requirements. The key point in photocatalysis is the developments of materials that efficiently transform the energy of the photons into chemical energy. In the case of semiconductors, upon photon absorption having energy higher than the band gap, promotion of one electron from the valence to the conduction band take place leading to a state of charge separation with the creation of conduction band electrons and valence band holes. Subsequently, electrons can migrate away from the site in where they where generated and eventually they can reach the surface of the semiconductor particles where they can interact with substrate adsorbed in the surface. Scheme 1.2. summarizes the difference steps, taking place upon photon absorption in a semiconductor. The two main limitations of a semiconductor as photocatalysts for solar fuels production are the low efficiency of charge separation due to the fast charge recombination of electrons and holes and the limitation derived from the band gap energy of the semiconductors. 
Scheme 1.2. Elemental steps of photocatalytic overall water splitting by semiconductors: (a) photon absorption (b) creation of a charge separated state (c) simultaneously reduction and oxidation of water to $\mathrm{H}_{2}$ and $\mathrm{O}_{2}$, respectively.

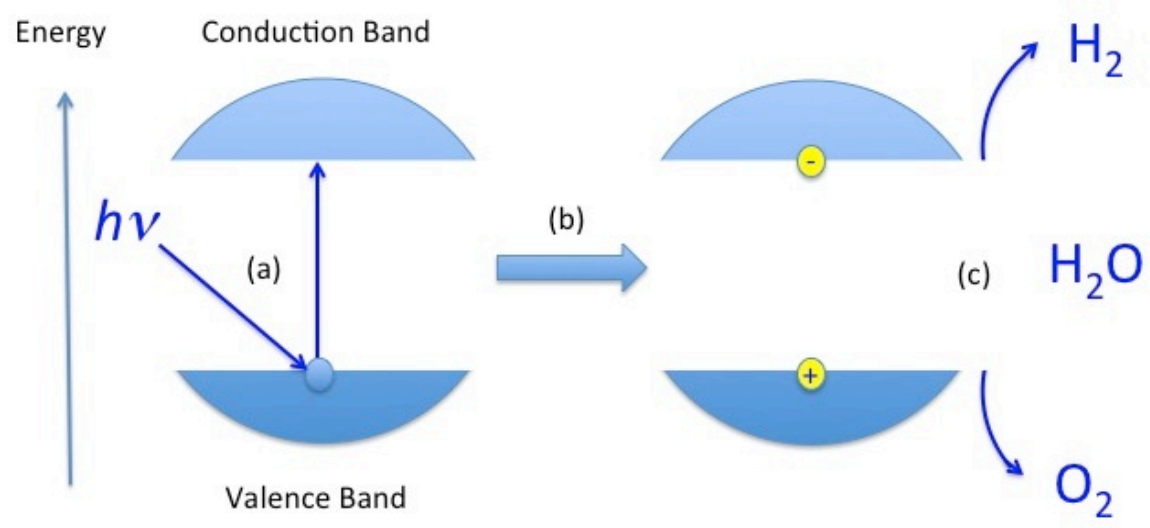

Since the pioneering studies of Fujushima and Honda ${ }^{[5]}$ (Scheme 1.3.) showing the photoelectrochemical water splitting by irradiation of a $\mathrm{TiO}_{2}$ electrode, titanium dioxide in different crystal phases and morphology has been the most widely studied photocatalyst for solar fuels production, including hydrogen generation from water.

Scheme 1.3. Representation of the mechanism involved in a photoelectrochemical cell.

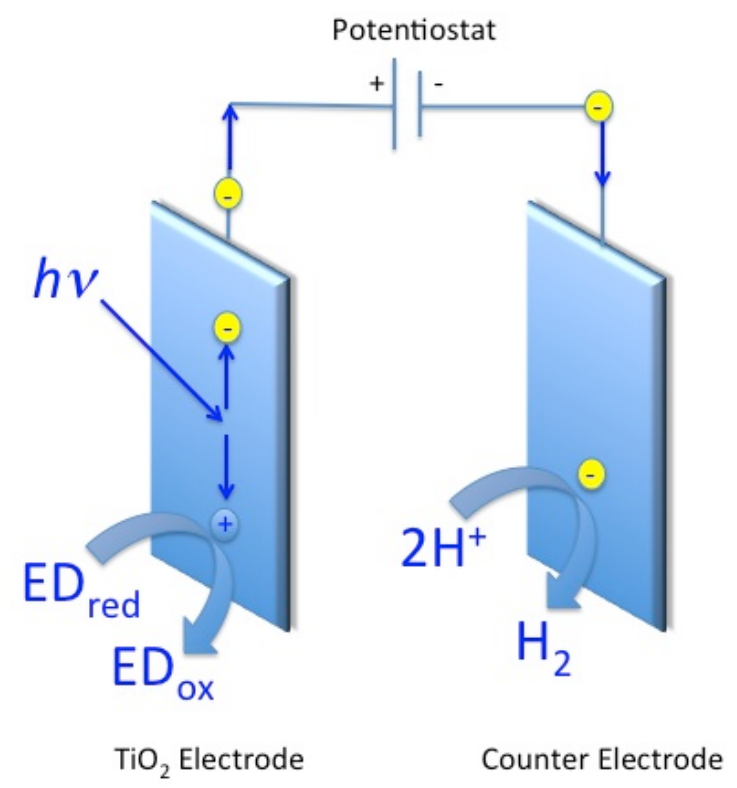


As shown in Scheme 1.4., overall water splitting is composed by two semireactions, corresponding to water oxidation and reduction. It is possible to decouple the two processes, by adding on purpose a sacrificial electron donor that makes the oxidation semireaction very favorable and fast, allowing to study the photocatalytic activity for hydrogen generation from water. Typical electron donors are tertiary amines and alcohols. These compounds as additives in the photocatalytic process make hole quenching very easy, the rate-determining step being hydrogen generation. In the present Thesis, the photocatalytic systems for hydrogen generation will be studied in the presence of an excess of methanol.

It had been found that the photocatalytic activity of $\mathrm{TiO}_{2}$ depends on the crystal phase and the crystallinity of the materials. In general, amorphous titania exhibits very low photocatalytic activity that has been attributed to the presence of defects in the structure that act as trapping sites of the charge carriers, favouring charge recombination process. Crystallinity in titania is generally achieved by annealing the solid at temperatures above $450{ }^{\circ} \mathrm{C}$, typically below $500{ }^{\circ} \mathrm{C}$. Of the different crystal phases, anatase, with a band gap of about $3.2 \mathrm{eV}$, is the phase that exhibits the highest photocatalytic efficiency. In contrast, brookite is almost inactive and there are contradictory reports on the photocatalytic activity of rutile that generally is considered to be much less efficient that anatase. ${ }^{[6]}$ Since anatase is the predominant crystal phase at temperature below of $600{ }^{\circ} \mathrm{C}$, care has to be taken to avoid phase transformation when submitting titania to thermal treatments. 
Scheme 1.4. A) Elemental step involved in water oxidation in presence of electron acceptor (a) photon absortion (b) creation of a charge separation state (c) Oxidation of $\mathrm{OH}^{-}$accompanied by the reduction of electron acceptor

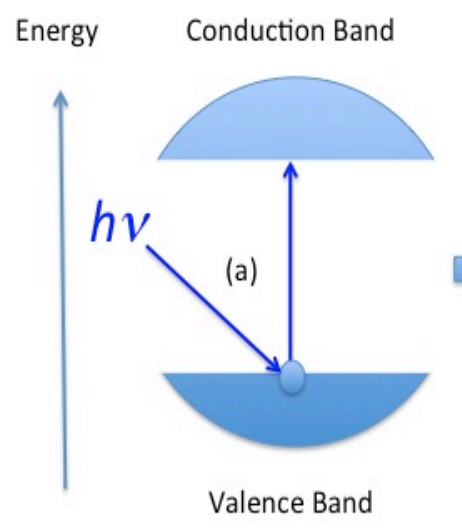

(b)

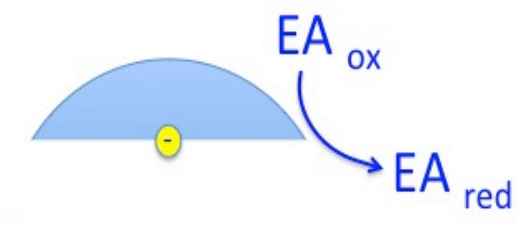

(c)

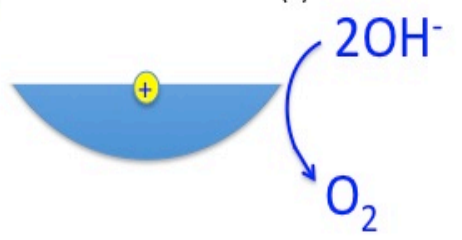

Scheme 1.4. B) Elemental step involved in water reduction in presence of electron donor (a) photon absorption (b) creation of a charge separated state (c) reduction of $\mathrm{H}^{+}$accompanied by the oxidation of electron donor.

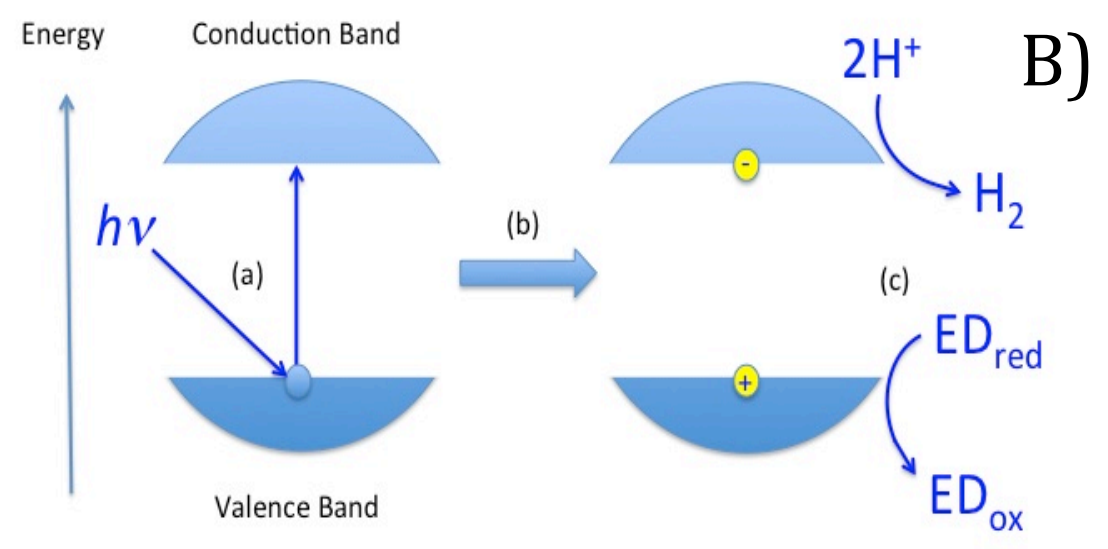


It has been determined that under appropriate conditions and UV light irradiation in the presence of platinum nanoparticles deposited on titania as cocatalyst, $\mathrm{TiO}_{2}$ can exhibit high efficiency for hydrogen generation that can be close to $40 \%$. In contrast under visible light irradiation, the efficiency of $\mathrm{TiO}_{2}$ for the same process in negligible. This lack of photocatalytic activity is due to the band gap energy of $\mathrm{TiO}_{2}$ anatase and the onset of light absorption that is about $300 \mathrm{~nm}$. This means that only photons of wavelength shorter of $380 \mathrm{~nm}$ can produce photoexcitation of titania. Since for solar fuels production excitation has to be performed by solar light reaching the Earth crust and this solar light contains only about $4 \%$ of the energy in the UV range and about $42 \%$ in the visible range, it is clear that the efficiency of $\mathrm{TiO}_{2}$ anatase for hydrogen production with solar light is very low, there being a need to increase the photoresponse toward the visible regions in order to enhance the efficiency of hydrogen generation. Figure 1.2. present the solar light spectrum measured at the Earth surface.

Figure 1.2. Solar irradiance spectrum above atmosphere and at the Earth surface.

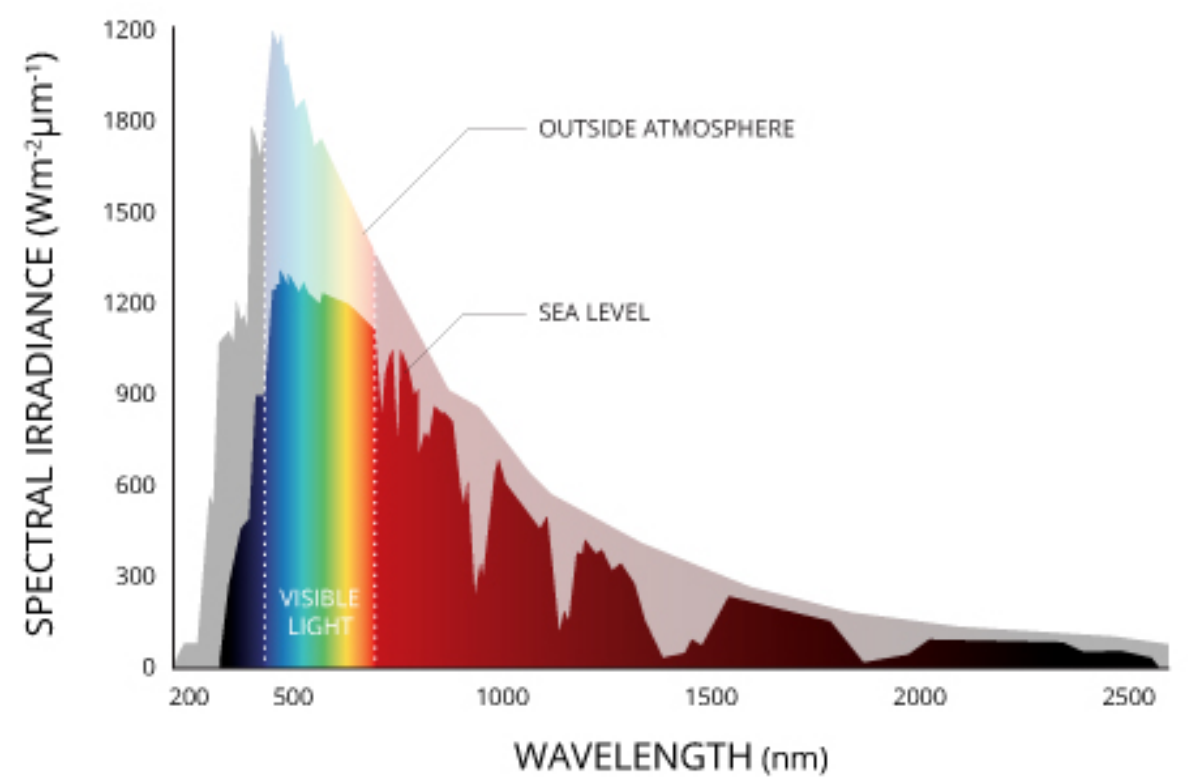

\subsection{Modified $\mathrm{TiO}_{2}$}

There are several possible modifications that had been reported to increase the photocatalytic activity of $\mathrm{TiO}_{2}$. As commented earlier, the presence of platinum nanoparticles deposited on the surface of $\mathrm{TiO}_{2}$, acting a cocatalyst in hydrogen generation is one of these modifications. It had been found that the efficiency for 
hydrogen generation of $\mathrm{TiO}_{2}$ can increase almost two orders of magnitude when a small percentage, about $1 \mathrm{wt} \%$, of platinum nanoparticles is anchored on the surface of the semiconductors (see Scheme 1.5.). It is generally assumed that upon charge separation by irradiation of semiconductors, electrons in the conduction band of $\mathrm{TiO}_{2}$ nanoparticles can migrate to the platinum nanoparticles that will act as a buffer of electrons and act as catalytic centre for hydrogen generations.

Scheme 1.5. Photocatalytic hydrogen generation using titania funcionalizated with platinum nanoparticles acting as cocatalyst. (a) photon absorption (b) creation of a charge separated state (c) migration of electron to platinum nanoparticles (d) reduction of $\mathrm{H}^{+}$mediated by platinum nanoparticle accompanied by the oxidation of electron donor.

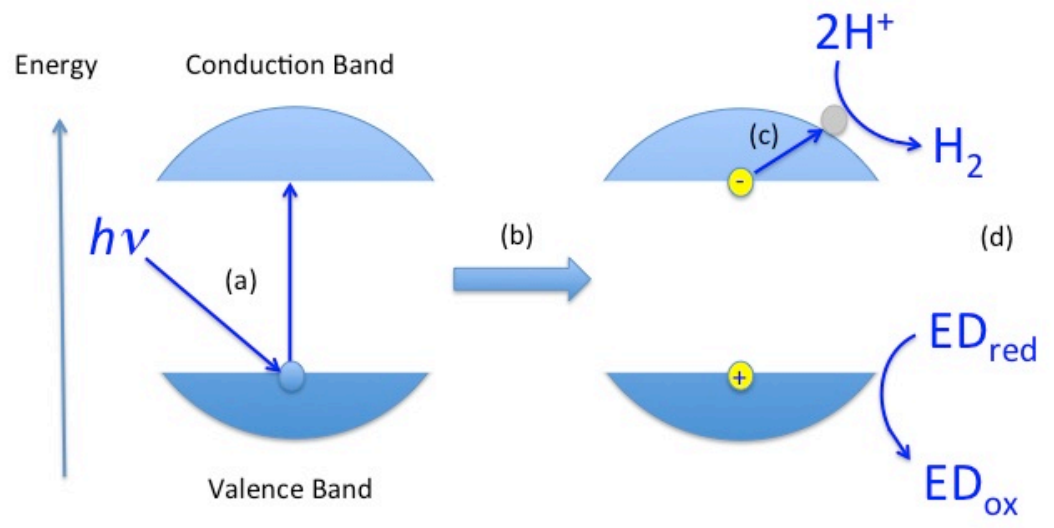

It is well known in catalysis that Pt and other noble metals have a large activity in many different reactions involving hydrogen, particularly hydrogenations of multiple bonds. One of the key points for increasing the efficiency of the hydrogen generation is the fact that electrons from the conduction band cross the surface toward the metal nanoparticle domains. This interfacial electron transfer increases significantly the efficiency of the photocatalytic process by reducing charge recombination in the semiconductor.

The concept of cocatalyst is being increasing applied in the current design of photocatalytic system. Typically noble metals are used to increase the rate of reduction promoted by conduction band electrons. On the other hand, $\mathrm{RuO}_{2}, \mathrm{Co}_{2} \mathrm{O}_{3}$ and other metal oxides have found to act as cocatalysts of the management of holes to effect the oxidation of substrates. 


\subsection{Au NPs supported on $\mathrm{TiO}_{2}$ as visible light photocatalyst for hydrogen generation.}

In this regard, our group has recently reported that gold nanoparticles supported on titania P25 (Au/P25) is one extremely efficient photocatalyst for hydrogen generation from water methanol mixtures. ${ }^{[7]}$ In the past Kamat and co-workers [8, 9] reported the photochemical response of mixtures of Au NPs and titania and found that laser exposure of these mixtures should lead to extremely high local temperatures as deduced by the size increase of Au NPs after the photochemical study. This "thermalization" of the energy of the photons was deduced by the fact that the temperature to melt $\mathrm{Au} \mathrm{NP}$ is about $600{ }^{\circ} \mathrm{C}[10]$. One of the physical proprieties of bulk metals that change dramatically in the nanoscale domain is the melting point. The melting point of bulk gold is reported to be $1064^{\circ} \mathrm{C}$, but upon reducing the particle size a dramatic decrease in this value takes place. However, in those experiments of Kamat, the $\mathrm{Au} / \mathrm{TiO}_{2}$ samples contain about 50 wt.\% of $\mathrm{Au}$ as nanoparticles of average size of about $50 \mathrm{~nm}$ [8, 9]. The increase in particle size of $\mathrm{Au}$ NPs upon irradiation with visible light at the surface plasmon band on gold was considered to derive from the melting of Au NPs and the agglomeration of the initial particles. It has to be, however, commented that although the $\mathrm{Au} / \mathrm{TiO}_{2}$ photocatalyst reported by us for $\mathrm{H}_{2}$ generation contain the same chemical constituents, the behaviour is completely difference due to the differences in the particle size and in the percentage of the components involved. Specifically, the $\mathrm{Au} / \mathrm{TiO}_{2}$ sample that exhibits high photocatalytic activity contain gold nanoparticle in weight percentage below 2 wt \%. In addition the particle size of gold in the efficient $\mathrm{Au} / \mathrm{P} 25$ photocatalysts is about $5 \mathrm{~nm}$ average. The preparation method followed to obtain the sample of $\mathrm{Au} / \mathrm{P} 25$ was initially reported by Haruta [11] and is denoted as "deposition-precipitation method". One of the characteristics of this deposition-precipitation method is strong metal support interaction that is reflected on the remarkable stability of Au NP particle size under (photo)catalytic reaction or under thermal annealing. With respect to the Kamat's study, ${ }^{[8,9]}$ the differences are notable and consist in low Au content, small Au NP particle size and strong interaction of $\mathrm{Au}$ with titania. As consequence, the photochemical behaviour of $\mathrm{Au} / \mathrm{P} 25$ is different to the one reported by Kamat ${ }^{[8,9]}$. As indicated in Scheme 1.6., the presence of $\mathrm{Au} / \mathrm{P} 25$, besides acting as cocatalyst of the 
photochemical reduction has also other role, particularly to introduce visible light photoresponse of the materials. This visible light photoresponse is proposed to derive from the presence of a visible absorption band due to the presence of $\mathrm{Au}$ NPs.

This visible absorption band is generally known as surface plasmon band and according to theory, it corresponds to the collective oscillation of free electrons confined in a cage of nanometric dimension corresponding to the Au NPs. The $\lambda_{\max }$ of the surface plasmon band depends of several factors including the morphology of $\mathrm{Au}$ NPs, the average dimension, and the extinction coefficient of the solid on which they are anchored. The position of the surface plasmon band can also depend of the charge density of Au NPs.

Scheme 1.6. Mechanistic proposal to explain the visible light response of $\mathrm{Au} / \mathrm{TiO}_{2}$ for the photocatalytic hydrogen generation from water. (a) photon absorption on plasmon band (b) creation of a charge separated state (c) reduction of $\mathrm{H}^{+}$to hydrogen accompanied by the oxidation of electron donor on gold nanoparticles.

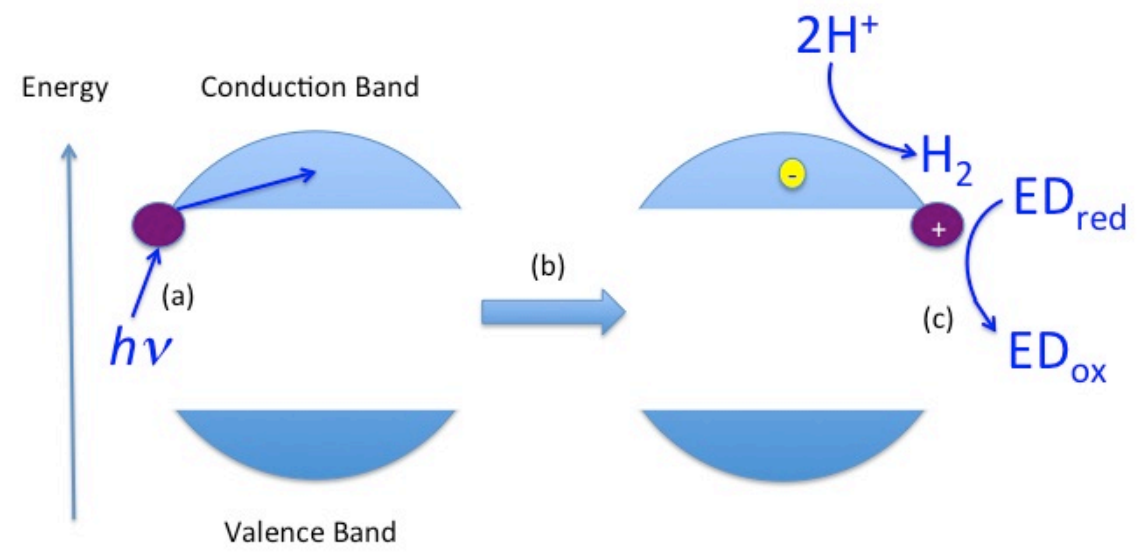

In particular, in the case Au/P25 the surface plasmon band is observed at $\lambda_{\max } 560$ $\mathrm{nm}$ with increasing intensity depending on the gold loading. Figure 1.3. presents diffuse reflectance UV-Vis optical spectrum of two samples of Au/P25. 
Figure 1.3. Diffuse reflectance of three samples of Au/P25 (M1, M2, M3) containing different loading of gold $(0.25,0.5$ and $1 \mathrm{wt} \%$ respectively). Inset $\mathrm{A})$ shows the magnification of the spectra in the characteristic region of gold plasmon band.

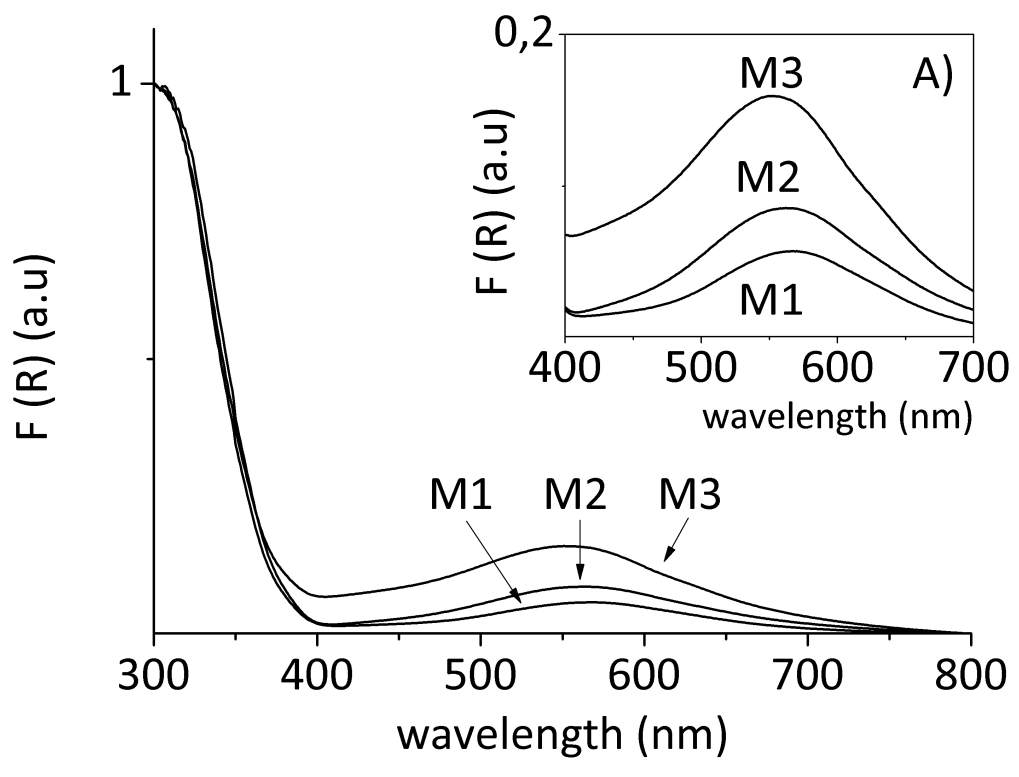

Photoexcitation of Au/P25 as photocatalyst in water methanol mixture with visible light result in the generation of hydrogen with a quantum efficiency at $550 \mathrm{~nm}$ estimated in about 5\%, that is among the highest ever reported and is particularly attractive considering the availability of P25 and the low content of gold. In this context, one of the chapters of the present Thesis would be aimed at determining the influence that Au alloyed with Pt can have on the photocatalytic activity.

One of the current topics in metal nanoparticles is the development of reliable procedures for the preparation of binary or ternary alloys of metal nanoparticles. These alloys can have random distribution of individual nanoparticles supported on a solid or can be real alloys in which a single nanoparticle contains both metals. In addition one possibility is to have core-shell structure of the nanoparticles, each of these situations can be characterized by TEM microscopy of the samples and could in principle lead to different results. The rationale is that real alloy nanoparticles should have electron density different from that of the individual components as consequence of the different electronegativity of the metals and this change in the electronic density could be reflected on changes on the 
photocatalytic activity. In this regard, chapter number 3 of this Thesis will describe the behaviour of Au-Pt alloy nanoparticles as sensitizer and cocatalyst of P25, particularly in comparison with control samples of the individual components. In a simplistic way it can be considered that the presence of Au would introduce visible light photoresponse as consequence of the presence of Au surface plasmon band and a certain catalytic activity. On the other hand, for UV irradiation had been determined that platinum is a better catalytic site for hydrogen evolution than gold. In this way, it could be predicted that it could be possible to observe an optimal percentage of platinum and gold in the alloys for reaching the highest activity in hydrogen generation. Also, it could be anticipated that the influence of alloy composition is different for UV than for visible light excitation of the system. This type of studies is of interest in the continuous search of more efficiently solar photocatalysts.

\subsection{Heterojunction photocatalysts.}

Related to the mechanism of operation of cocatalysts, it also been found that "heterojunction" assemblies can favour the efficiency of a photocatalyst by reducing charge recombination. The term heterojunction refers to the situation in where an intimate contact between two different semiconductors allows electron transfer from the semiconductor having the higher conduction band energy to the semiconductor with conduction band energy lower. The concept of heterojunction derives from the operation of electrostatic fields and Schottky barriers at the interface of the two semiconductors that should favour electron transfer in one direction and disfavour the electron transfer in the opposite direction. One very well known example of advantageous heterojunctions in terms of increasing the catalytic efficiency is commercial P25 titania that is known to be formed by a combination of $80 \%$ anatase and $20 \%$ rutile.

The main difference between the concept of heterojunction and metal nanoparticles as cocatalyst is the conducting or semiconductor nature of the components of the system. In the case of heterojunction the two components should be semiconductors. In the chapter number 5 of the present Thesis we also explore the potential of a system based on the concept of heterojunction between two types of titanium-containing semiconductors for photocatalytic hydrogen 
generation. While most of the examples, except P25, illustrating the concept of heterojunction refer to different components, it would be also important to show that two different semiconductors of the same transition metal can also build a heterojunction, exhibiting enhancement of the photocatalytic activity. In this regard, one of the favourite heterojunctions is the one based on quantum dots, and particularly CdS, deposited on titania. ${ }^{[12]}$ In the present Thesis, we would like to use heterojunction with two different titanium-containing solids, in particular titanate in combination with titanium dioxide. The strategy based on heterojunction also allowed implementing cocatalysts to further increase the photocatalytic activity and for this reason we would prepare a system of titanate and titanium dioxide heterojunction containing noble metal nanoparticles. The heterojunction between titanate and titanium dioxide nanoparticles derive from the different band energies and band gaps between the two materials. Titanates are layered materials that can be exfoliated at the level of single titanium atoms thickness. These layers present metal vacancies that also influence the position of the bands and the band gap energy.

In fact one of the well established methodology to control the photocatalytic efficiency of titanium materials is by modifying the morphology of the particles. Morphology and size of titanium particles are known to play a key role on the photocatalytic activity. In this context it had been found that small $\mathrm{TiO}_{2}$ clusters undergo a blue shift in the position of the absorption band and an increase in the band gap energy as consequence of the confinement and the quantum size effect. It has been found that this nanometric $\mathrm{TiO}_{2}$ clusters of a few $\mathrm{Ti}$ atoms can exhibit extremely high photocatalytic efficiency for certain processes including photocatalytic $\mathrm{CO}_{2}$ reduction [13], but only when they are excited with photons of the appropriate wavelength. The blue shift of the absorption band required for the excitation of this cluster photons with wavelength below $350 \mathrm{~nm}$ that are absent in solar light. As consequence, even though the photocatalytic efficiency of these $\mathrm{TiO}_{2}$ clusters can be high, they require excitation with artificial UV light, making the process uninteresting from the point of view of solar fuel production.

Also by transient absorption spectroscopy, it was found that needles and nanotubes of $\mathrm{TiO}_{2}$ exhibit a large charge mobility in the direction of the long axis [15]. This charge mobility in preferential directions has led to the conclusion that in 
crystals of $\mathrm{TiO}_{2}$ exposing different facets, electrons and holes could be located differently [15].

Another examples of remarkable influence of the morphology of the particles on the photocatalytic activity are the recent procedures of $\mathrm{TiO}_{2}$ preparation with aqueous solution of hydrochloric [15] or hydrofluoric ${ }^{[16]}$ acid that produce particles with specific truncated prism morphology and high photocatalytic activity. Of interest in the present case, is the fact that $\mathrm{small}^{\mathrm{TiO}_{2}}$ nanoparticles of $50 \mathrm{~nm}$ or below are among the most efficient titania photocatalyst. For this reason any preparation procedure rendering small $\mathrm{TiO}_{2}$ nanoparticles with high crystallinity could be of large interest in photocatalysis if this results in an enhanced photocatalytic efficiency.

A recent strategy that has been disclosed recently as a way to increase the photocatalytic efficiency of $\mathrm{TiO}_{2}$ has been amorphization of a few external layers of $\mathrm{TiO}_{2}$ nanoparticles [18]. This surface and subsurface modification of the particles can be achieved by thermal treatment under hydrogen atmosphere. It has been found that this thermal annealing under reducing conditions do not influence the crystallinity of the $\mathrm{TiO}_{2}$ nanoparticles, but, however, produces a remarkable change in the visual appearance of titania samples that become black ("black titania").[18] Conventional characterization techniques show that hydrogen annealing does not affect most of the physical properties of $\mathrm{TiO}_{2}$ such a infrared and Raman spectra, BET surface area, particles size and other properties that do not change with respect conventional $\mathrm{TiO}_{2}$ samples. With respect to UV-Vis absorption spectroscopy, hydrogen annealing introduces a broad absorption without any relative maxima growing in intensity toward short wavelengths that has been attributed to the influence of the surface amorphous layer. 
Scheme 1.7. Schematic representation of the process used to prepare black titania

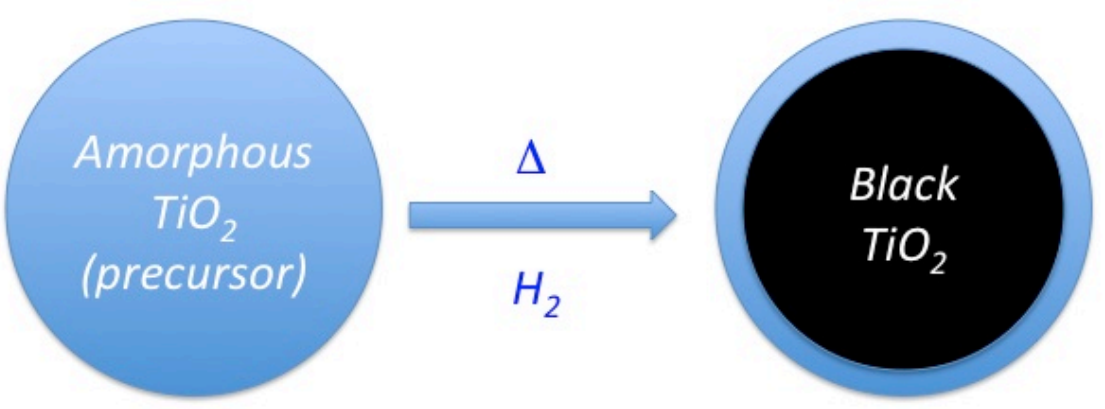

A remarkable effect of hydrogen annealing is an enhancement of the photocatalytic activity of black titania for $\mathrm{H}_{2}$ generation with visible light upon addition of platinum nanoparticles as cocatalyst to the material. In the present thesis we have also developed the concept of black titania in a different way to the one that has been reported. Specifically in chapter 4 we will study the influence that the hydrothermal treatment and the $\mathrm{pH}$ of the solution have in the UV-Vis absorption spectra as another procedure to obtain of $\mathrm{TiO}_{2}$. Also the influence of hydrogen annealing on the phase transformation from titanate to $\mathrm{TiO}_{2}$ nanoparticles will be the subject of study in this chapter. The rationale behind it is that hydrogen annealing has higher influence on the surface proprieties when starting from titanate layer due to the high accessibly of the sites and in the process of transformation of this titanate sheet into titania nanoparticles.

\subsection{Framework Phosphates as photocatalysts.}

In the previous comments about photocatalysis, titanium dioxide has been highlighted as the most widely used and efficient photocatalyst. We have already commented that it would be important to find alternative efficient photocatalysts that could exhibit similar or even higher photocatalytic activity than titania.

Alternative photocatalysts should try to overcome the mayor limitations of titania in terms of visible light photoresponse and low efficiency of charge separation. In literature there are some other photocatalysts that had been evaluated for hydrogen production and in general preparation of solar fuels. [19] The last include 
other metal oxides, metal sulphides, mixed oxides with perovskite or layered structured and many other materials such as nitrides, oxynitrades and other combination of metals with elements of the group V such a arsenide phosphide and so on. In this context, it has been recently reported by Nocera's group [20] that cobalt phosphate is highly abundant and efficient, not noble metal containing catalyst for electrochemical oxygen evolution. Framework phosphate materials can be easily prepared by precipitation of metal ion solutions or by solvothermal procedures, it is surprising to know that they have been almost completely ignored as photocatalysts. Metal phosphates in the form of open framework are materials in where the crystal structure is defined by metal-oxygen-phosphorus bonds and contain some organic amine in the structure. These open metal frameworks have the advantage of lower framework density, higher accessibility of the sites and easy and reliable preparation procedures. Particularly notable is the lack of information on the photocatalytic activity of titanium phosphate, particularly in the field of $\mathrm{H}_{2}$ generation. Considering the photocatalytic activity of titanium dioxide, titanium perovskite and other titanium containing solids, it is in a certain way surprising that up to now the possibility of developing framework titanium phosphate as photocatalyst has been completely ignored.

In addition, in a precedent it is has been reported the preparation of mixed valence $\mathrm{Ti}^{3+} / \mathrm{Ti}^{4+}$ open-framework phosphate. [21] Coming back to the case of titanium dioxide, it has been reported that $\mathrm{Ti}^{3+}$ could act as a metal doping centre in $\mathrm{TiO}_{2}$, introducing visible absorption band in the optical absorption spectrum in the material and also visible light photoresponse.[15] $\left(\mathrm{Ti}^{3+}\right) \mathrm{TiO}_{2}$ exhibits as consequence of the presence of $\mathrm{Ti}^{3+}$ higher photocatalytic activity, particularly under visible light illumination, for hydrogen generation from water. ${ }^{[15]}$ It could be anticipated that a similar behaviour could be expected for mixed valence $\mathrm{Ti}^{3+} / \mathrm{Ti}^{4+}$ phosphate, but no experimental data in this regard has been reported so far and, therefore, is would be of much interest to determine if is the case or not. Besides mixed valence $\mathrm{Ti}^{3+} / \mathrm{Ti}^{4+}$, open-framework phosphates also offer the possibility of prepare other mixed metal materials in where two or more transition metals are present in a random distribution in the solid. One possibility that is worth to study is the case in where one of the metals in the binary composition is in much lower proportion than the other, since this case would represent doping of a metal 
phosphate with a different metal. As in the case of $\mathrm{TiO}_{2}$, this situation can also introduce some empty orbitals below the conduction band level that could, on one hand, shorten the band gap energy of the material and, on the other hand, can also decrease electron-hole recombination, that is main energy waste deactivation pathway in photocatalysis.

According to these ideas, it is of general interest to show whether or not metal phosphates behave as semiconductors. In particular of the vast number of possibilities, we have chosen as the most relevant to determine the semiconductor activity, the case of titanium phosphate and iron phosphate. Accordingly, chapters 6 and 7 with deal with the photocatalytic activity of mixed valence $\mathrm{Ti}^{3+} / \mathrm{Ti}^{4+}$ and doped iron phosphate, respectively.

The reasons for this selection are because titania and hematite $\left(0 \mathrm{TiO}_{2}\right.$ and $\mathrm{Fe}_{2} \mathrm{O}_{3}$ ) are two materials that have attracted considerable attention as photocatalysts for hydrogen generation from water. While in the case of $\mathrm{TiO}_{2}$ the efficiency of $\mathrm{H}_{2}$ generation in the presence of sacrificial agent is very high upon UV irradiation, in the case of hematite, the visible light photoresponse for this process can be very high ${ }^{[22]}$ but requires a bias voltage to increase the conduction band potential over the value required for water reduction.

To establish the semiconductor properties of metal phosphates it is necessary to prove that the material exhibits simultaneously two properties [23]. One of them is the generation of charge separate state upon absorption of photons with energy larger of the band gap energy. The second propriety of a material to be considered semiconductor is mobility of at least one of the charge carriers, either electrons or holes, depending on the $\mathrm{n}$ or $\mathrm{p}$ nature of the semiconductor. In the present Thesis we will address this issue obtaining experimental evidence in support of the semiconducting behaviour of metal phosphates, besides to determine to ability to generate hydrogen from water in the presence of sacrificial electron donors. The generation of charge separate states in a material can be firmly established by transient absorption spectroscopic techniques. In time resolved spectroscopy, the sample is excited with a short laser pulse of the adequate wavelength and after excitation is monitored for the change in its absorption spectra and the temporal profile of the transient signal. The nature of the transient and its assignment to electron and/or holes can be based on its behaviour in the presence of quenchers 
that can give or accept electrons from the transient. Typical quenchers are molecular oxygen or nitrogen oxide as acceptors of conduction band electrons and methanol or triethylamine as acceptor of positive holes. If a transient species corresponds to the state of charge separation, its decay should be altered by the presence of these quenchers.

Charge mobility and the density of charge carriers in semiconductor can be determined by impedance spectroscopy as well as determination of the current generated upon irradiation in electrodes submitted to external bias. In impedance spectroscopy one conductive electrode is coated by the materials and the resulting electrodes of the solid under study is immersed in an electrolyte solution and submitted to an alternate voltage, while the frequency is scanned from low values $(\mathrm{Hz})$ to high values $(\mathrm{MHz})$. In the high frequency range the response of the material to polarization is related to the number of carrier and the distance they can travel. From the Nyquist plot obtained for the impedance spectroscopy measurements and the fitting the electrode behaviour to a model electric circuit, the values of the energy of the conduction band can be determined.

In the photocurrent measurements a transparent conductive electrode coated with the material under study is polarized to a certain voltage and submitted to irradiation, monitoring the dark and the current under illumination. The constant polarization voltage in each measurement is scanned from the negative to positive voltages of direct current. The change in the polarity to positive to negative in the dark and illuminated currents take place a certain bias voltage that corresponds to the voltage of the flat band potential of the conduction band and the change to positive to negative or viceversa indicate the $\mathrm{n}$ or $\mathrm{p}$ nature of the semiconductor.

In summary the general objective of this thesis is to study the photocatalytic response for hydrogen generation of a series of materials trying to increase the efficiency for this process by applying different methodologies. These methodologies include heterojunction between different titanium semiconductors, binary metal nanoparticles as cocatalyst, amorphization of external surface to introduce visible light photoresponse or the use of metal phosphate as photocatalysts. 


\subsection{References.}

[1] Dresselhaus, M., et al., Basic Research Needs for the Hydrogen Economy. 2003.

[2] A. Kogan, Int. J. Hydrogen Energy, 1998, 23, 89.

[3] Steinfeld, A., Solar Energy, 2005, 78, 603-615.

[4] Nakamura, T. Solar Energy 1977, 19, 467-475.

[5] A. Fujishima, K. Honda Nature, 1972, 238, 37 - 38.

[6] Q. Sun, Y. Xu, Phys. Chem. C, 2010, 114, 18911-18918.

[7] C. Gomes Silva, R. Juarez, T. Marino, R. Molinari, H. Garcia, J. Am. Chem. Soc. 2011, 133, 595.

[8] Jakob, M.; Levanon, H.; Kamat, P. V. Nano Lett. 2003, 3, 353-358.

[9] Kamat, K.; Maeda, K.; Lu, D. L.; Kako, Y.; Domen, K. Chem. Phys. Lett. 2009, 470, 90-94

[10] Kamat, P.V, Journal of Physical Chemistry B, 2002, 106, 7729-7744.

[11] G. R. Bamwenda, S. Tsubota, T. Kobayashi and M. Haruta, J. Photochem. Photobiol., A, 1994, 77, 59-67.

[12] Y. Lai, Z. Lina, D. Zhenga, L. Chi, R. Dua, C. Lin Electrochimica Acta, 2012, 79, 175-181.

[13] Mori, K.; Yamashita, H.; Anpo, M. P, RSC Adv. 2012, 2, 3165-3172.

[14] H. Xu, S. Ouyang, P. Li, T.Kako, J. Ye ACS Appl. Mater. Interfaces, 2013, 5, 1348-1354

[15] Zuo, F. Bozhilov, K. Dillon, R. J. Wang, L. Smith, P. Zhao, X. Bardeen, C. Feng, P., Angew. Chem., Int. Ed. 2002, 51, 6223-6226, S6223/1-S6223/9.

[16] M. V. Dozzi, E. Selli, Catalysts 2013, 3, 455-485

[17] A. Primo, M. Liebel, F. Quignard, Chem. Mater. 2009, 21, 621.

[18] Chen, X. Liu, L. Yu, P. Y. Mao, S. S., Science 2011, 331, 746-750.

[19] A. Kudo, Y. Miseki, Chem. Soc. Rev., 2009,38, 253-278.

[20] Kanan, M. W. Surendranath, Y. Nocera, D. G., Chem. Soc. Rev. 2009, 38, 109114.

[21] Ekambaram, S. Sevov, S. C., Angew. Chem., Int. Ed. 1999, 38, 372-375.

[22] U. Bjorksten, J. Moser, M. Gratzel, Chemistry of Materials 1994, 6, 858.

[23] Gomes Silva, C., A. Corma, H. Garcia, Journal of Materials Chemistry, 2010, $20,3141-3156$. 
Chapter 2

objectives. 


\section{Chapter 2 - Objectives.}

\subsection{Objectives.}

As commented at the end of previous section, this Thesis is focused on the increase in the efficiency of $\mathrm{H}_{2}$ generation from water in the presence of sacrificial agents by applying different methodologies. Two types of materials, either based on titanium oxide or on metal phosphates will be studied in the present Thesis for this process. Specifically the objectives of the present Thesis are the following:

1. Optimization of Au NPs as cocatalyst of $\mathrm{TiO}_{2}$ for hydrogen generation by incorporation in the metal nanoparticle of Pt. The objective is to form binary Au-Pt alloys in the form of NPs in order to increase efficiency in the material for hydrogen generation. In these samples of binary Au-Pt alloy nanoparticles supported on $\mathrm{TiO}_{2}$ sample it is also important to establish the influence of the irradiation wavelength on the photocatalytic activity.

2. Evaluation of the photocatalytic activity of titanium containing semiconductors either as nanotubes or nanoparticles that are obtained by hydrothermal synthesis under strong basic conditions and subsequent calcination under hydrogen atmosphere at various temperatures. The purpose is to establish the influence that the possible absorption band in the visible region as well as change in the morphology can play on the activity of these materials.

3. Preparation of heterojunctions using two different forms of titanium containing materials and evaluation of their photocatalytic activity both in the presence and absence of metal nanoparticles as cocatalysts. One of the semiconductors forming part of the heterojunction would be $\mathrm{TiO}_{2}$ nanoparticle, while the other component would be titanate nanotubes prepared by hydrothermal synthesis. The performance of this heterojunction as a function of the amount of one semiconductor with respect to other would be evaluated. 
4. Evaluation of the photocatalytic activity of un-doped and doped mixed valence open framer $\mathrm{Ti}^{3+} / \mathrm{Ti}^{4+}$ phosphate for hydrogen generation from water in the presence of sacrificial agents. Besides activity measurements, experimental evidences of behaviour of this type of phosphate as semiconductor will also be obtained by a combination of transient absorption spectroscopy and photoelectrochemical measurements.

5. Characterization of open-framework iron phosphate as semiconductor and evaluation of its photocatalytic activity for hydrogen generation from water methanol mixtures. Determination of the influence of doping with heteroatoms and preparation of mixed iron metal phosphate including other transition metal and the evaluation of their photocatalytic activity.

In the case that all these objectives are achieved, the present Thesis will represent and step forward in the field of solar fuel production and more specifically generation of hydrogen from aqueous solutions. Furthermore, novel photocatalyst different from conventional titanium oxide with considerable promise with respect to the possibility of doping will also be implemented. 
Chapter 3

Influence of the irradiation wavelength on the photocatalytic activity of Au-Pt nanoalloys supported on $\mathrm{TiO}_{2}$ for hydrogen generation from water. 
Chapter 3 - Influence of the irradiation wavelength on the photocatalytic activity of $\mathrm{Au}-\mathrm{Pt}$ nanoalloys supported on $\mathrm{TiO}_{2}$ for hydrogen generation from water.

\subsection{Introduction.}

In the context of renewable energy resources to fossil fuels, photocatalytic hydrogen generation by solar light is gaining increasing interest due to the possibility of using hydrogen as a clean transportation fuel.[1-7] For this purpose, modified $\mathrm{TiO}_{2}$ is the most widely used photocatalyst. ${ }^{[1,8-13]}$ The main problem to be overcome is the negligible visible light photoresponse of plain $\mathrm{TiO}_{2} \cdot{ }^{[1,5,8,14-24]}$ Among the various possibilities to enhance the visible light photocatalytic activity of $\mathrm{TiO}_{2}$, one that has attracted considerable interest in recent times is the use of gold nanoparticles (Au NPs) as a light harvester and a photo sensitizer for $\mathrm{TiO}_{2} \cdot[17,18,21]$

Compared to conventional metal doping, the use of $\mathrm{Au} / \mathrm{TiO}_{2}$ presents the advantage of reliable preparation procedures, high durability due to the noble metal character of gold and high visible light photocatalytic activity due to the broad absorption originating from the surface plasmon band of Au NPs expanding from 400 to $700 \mathrm{~nm}$. In this $\mathrm{Au} / \mathrm{TiO}_{2}$ photocatalyst, $\mathrm{Au}$ may play simultaneously several roles including light absorption and photosensitization of $\mathrm{TiO}_{2}$ as well as acting as a catalytic centre to yield the photoproducts.[17,18,21]

As we have already commented in the introduction, thus it is of interest to assess the relative photocatalytic activity of Au nanoalloys. In particular, in the present manuscript we are concerned with providing comparative data for the photocatalytic activity of $\mathrm{TiO}_{2} \mathrm{P} 25$ modified by $\mathrm{Au}-\mathrm{Pt}$ nanoalloys. The presence of $\mathrm{Pt}$, with higher catalytic activity than $\mathrm{Au}$ in thermal hydrogenation ${ }^{[25]}$ and oxidation, ${ }^{[26]}$ can lead to more efficient photocatalytic activity if the controlling efficiency limiting steps are related to the role of metal NPs as catalysts. On the other hand, the presence of Pt could have a detrimental effect on the photocatalytic activity due to the fact that Pt has no surface plasmon band absorption and the presence of this metal in the nanoalloy could have adverse effects on the light absorption and on the electron injection events of the photocatalytic cycle. 
In the present chapter we provide a comparison of the photocatalytic activity of $\mathrm{Au}-\mathrm{Pt}$ nanoalloys under visible, UV or simulated solar light irradiation, both in the presence and absence of methanol as a sacrificial electron donor.

For visible light irradiation we used purposely a $532 \mathrm{~nm}$ laser excitation (green monochromatic light) to ensure excitation near the $\mathrm{Au}$ surface plasmon band absorption maxima $(560 \mathrm{~nm})$. In recent related precedents, photocatalytic hydrogen production using $\mathrm{Au}-\mathrm{Pt} / \mathrm{TiO}_{2}$ photocatalysts has been studied using UV or solar light excitation and it was concluded that the combination of the $\mathrm{Au}-\mathrm{Pt}$ nanoalloy (atomic ratio $1: 1$ ) exhibits the highest photocatalytic activity compared to the samples containing each of the noble metal independently. ${ }^{[27,28]}$ This positive influence exerted by Pt is also in line with previous work using UV light that established the higher activity of $\mathrm{Pt}$ over $\mathrm{Au}$ for hydrogen generation.[29] Considering these precedents in which apparently the presence of Pt increases the activity of $\mathrm{Au}$ under UV light and on the other the fact that Pt has no surface plasmon band that can be used to photosensitize $\mathrm{TiO}_{2}$, it would be of interest to undertake a study of the photocatalytic activity of $\mathrm{Au}-\mathrm{Pt}$ alloys under visible light to clarify if, also under these conditions, the presence of $\mathrm{Pt}$ increases the photocatalytic activity.

\subsection{Results and discussion.}

\subsubsection{Sample preparation and characterization.}

This chapter first we will comment on the preparation and characterization of $\mathrm{Au}$ Pt alloy nanoparticles by analysis, microscopy and spectroscopy and then we will describe the photocatalytic activity of these material for hydrogen generation under visible light as well as UV light and simulated sunlight.

For the present study aimed at determining the relative photocatalytic activity of $\mathrm{Au}-\mathrm{Pt}$ nanoalloys for visible light hydrogen generation, we have prepared six samples, all of them at the same overall metal loading in which part of the Au has been replaced by an increasing proportion of Pt up to 50\%. The list of the samples prepared and the main analytical and spectroscopic data are summarized in Table 3.1. In these samples the $\mathrm{Au}-\mathrm{Pt}$ nanoalloys are deposited on commercially available P25 titania, which is a common reference material in the field and 
exhibits high photocatalytic activity for hydrogen generation under UV light irradiation.

In addition, all the samples were prepared by the well-known deposition precipitation method. This procedure consists of two steps, the first of which is the adsorption of gold species from $\mathrm{AuCl}_{4}{ }^{-}$aqueous solutions at controlled $\mathrm{pH}$ on the surface of hydroxylated titania (deposition). Subsequently, nucleation and generation of metal NPs is accomplished by Au reduction under various conditions. In our case the samples were treated by a hydrogen flow at $300{ }^{\circ} \mathrm{C}$ in order to effect the reduction of $\mathrm{Au}^{3+}$ and $\mathrm{Pt}^{4+}$ to the corresponding metal state. The overall loading (about $0.25 \mathrm{wt} \%$ ) was selected in the range where previously the highest visible light photocatalytic activity was measured.

Table 3.1. Au-Pt/ $\mathrm{TiO}_{2}$ samples tested in this study and their corresponding total metal loading and Au percentage

\begin{tabular}{|l|l|l|}
\hline Sample code & Total metal content $(\mathrm{a})$ & $\mathrm{Au}(\%)$ \\
\hline S1 & 0.247 & 100.0 \\
\hline S2 & 0.234 & 78.2 \\
\hline S3 & 0.229 & 72.2 \\
\hline S4 & 0.255 & 65.0 \\
\hline S5 & 0.257 & 50.0 \\
\hline S6 & 0.264 & 31.0 \\
\hline S7 & 0.233 & 0.0 \\
\hline
\end{tabular}

(a) Combined weight of $\mathrm{Au}$ and Pt metals divided by total sample weight in percentage.

Deposition of Au-Pt nanoparticles on P25 titania does not change the Raman spectra or XRD of the solid that correspond basically to that expected for P25 without reflecting the presence of a minor amount of noble metal. The percentage of $\mathrm{Au}$ and $\mathrm{Pt}$ on the solid was determined by ICP analysis using calibrated standards. Transmission electron microscopy images show the presence of small metal nanoparticles of about $1.5 \mathrm{~nm}$ diameter on the titania surface. No notable 
differences in the particle size distribution were observed for the various samples having different Pt percentage. As we will comment below, this is the average size corresponding to $\mathrm{Au}-\mathrm{Pt}$ particles, since XPS indicate the formation of $\mathrm{Au}-\mathrm{Pt}$ alloys. Figure 3.1. shows selected TEM micrographs of the samples to illustrate the morphology and size of the noble metal nanoparticles.

Figure 3.1. TEM images of S1 (left) and S4 (right) samples and their corresponding particle size histograms.
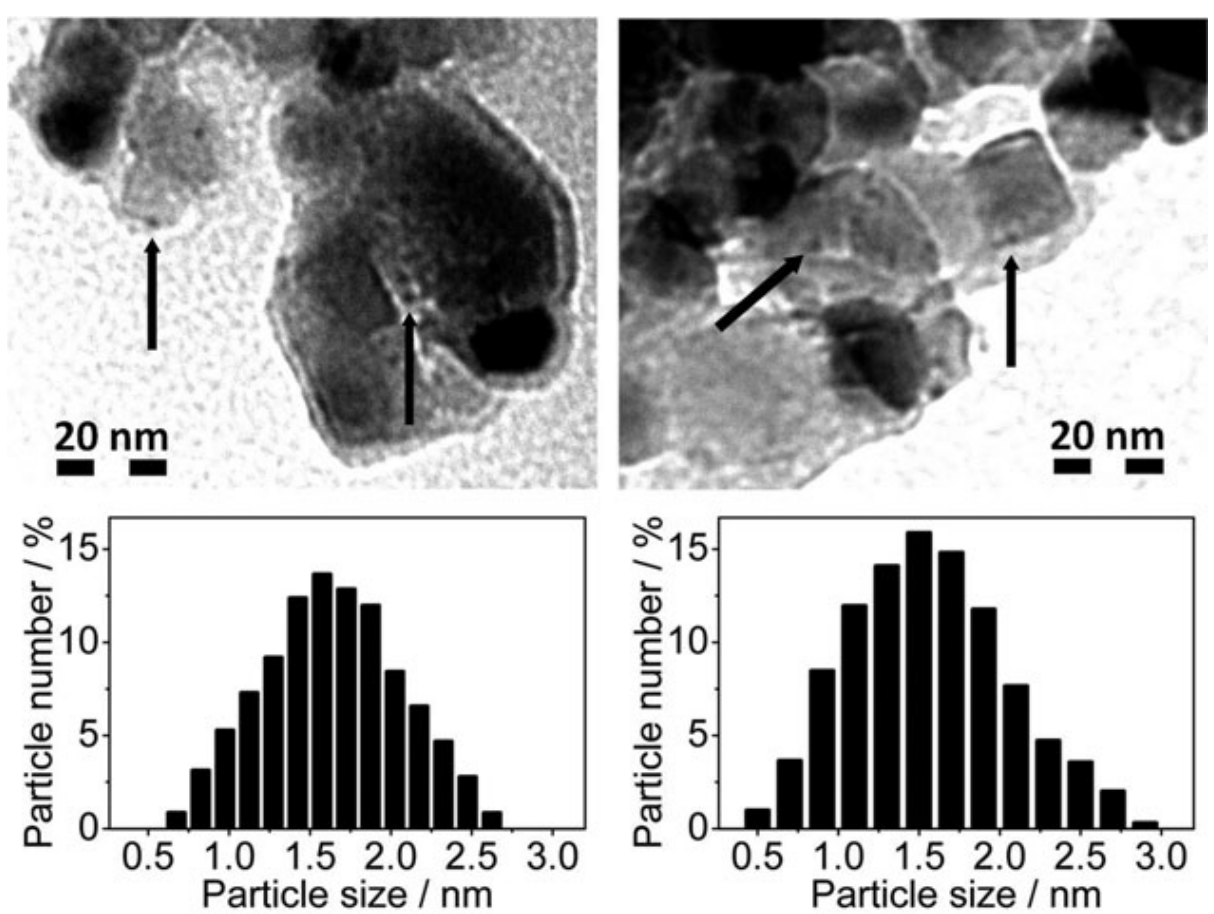

Metal alloys can be studied by EDS analysis that allows us to determine the spatial distribution of both metals on the sample. However, since $\mathrm{Pt}$ and $\mathrm{Au}$ are neighbours in the periodic table, this technique does not allow unfortunately to map out the distribution of both metals. In order to address if our samples contain independent $\mathrm{Pt}$ and $\mathrm{Au}$ nanoparticles or if real $\mathrm{Au}-\mathrm{Pt}$ nanoalloys are present we used XPS analysis by determining the binding energies of the components of experimental Au4f7/2 and Pt4f7/2 peaks. XPS probing a depth of about $30 \mathrm{~nm}$ of the compressed powdered photocatalyst wafer (thickness of about $800 \mathrm{~mm}$ ) really gives an indication of the average distribution of $\mathrm{Au}$ and $\mathrm{Pt}$ atoms in the sample considering that $\mathrm{TiO}_{2}$ particles are about $50 \mathrm{~nm}$ in diameter. 
The fact that we have observed significant shifts in the binding energy values for the Pt 4f7/2 (76.4 and $75.5 \mathrm{eV}$ for S5 and S6, respectively) as well as for the two components of $\mathrm{Au} 4 \mathrm{f} 7 / 2$ (83.9 and $88.4 \mathrm{eV}$ for S5 with respect to 84.7 and $88.8 \mathrm{eV}$ for S6) strongly suggests the formation of real alloys between $\mathrm{Au}$ and $\mathrm{Pt}$ at the nanometric scale. Thus, the XPS technique shows that the predominant distribution of $\mathrm{Au}$ and $\mathrm{Pt}$ forms nanoalloys. Figure 3.2. shows the experimental $\mathrm{Au}$ $4 \mathrm{f7} / 2$ peaks recorded for samples S5 and S6, together with the deconvolution components and the estimated binding energies.

All the samples exhibit the characteristic pink color indicating the presence of $\mathrm{Au}$ NPs. In this case, this surface plasmon band should arise from the $\mathrm{Au}-\mathrm{Pt}$ nanoalloys that are the predominant NPs or from a low proportion of individual $\mathrm{Au}$ NPs not detectable by XPS.

Figure 3.2. XPS Au 4f7/2 peak recorded for samples S5 and S6 together with its deconvolution in individual components based on the best fitting of the experimental data.

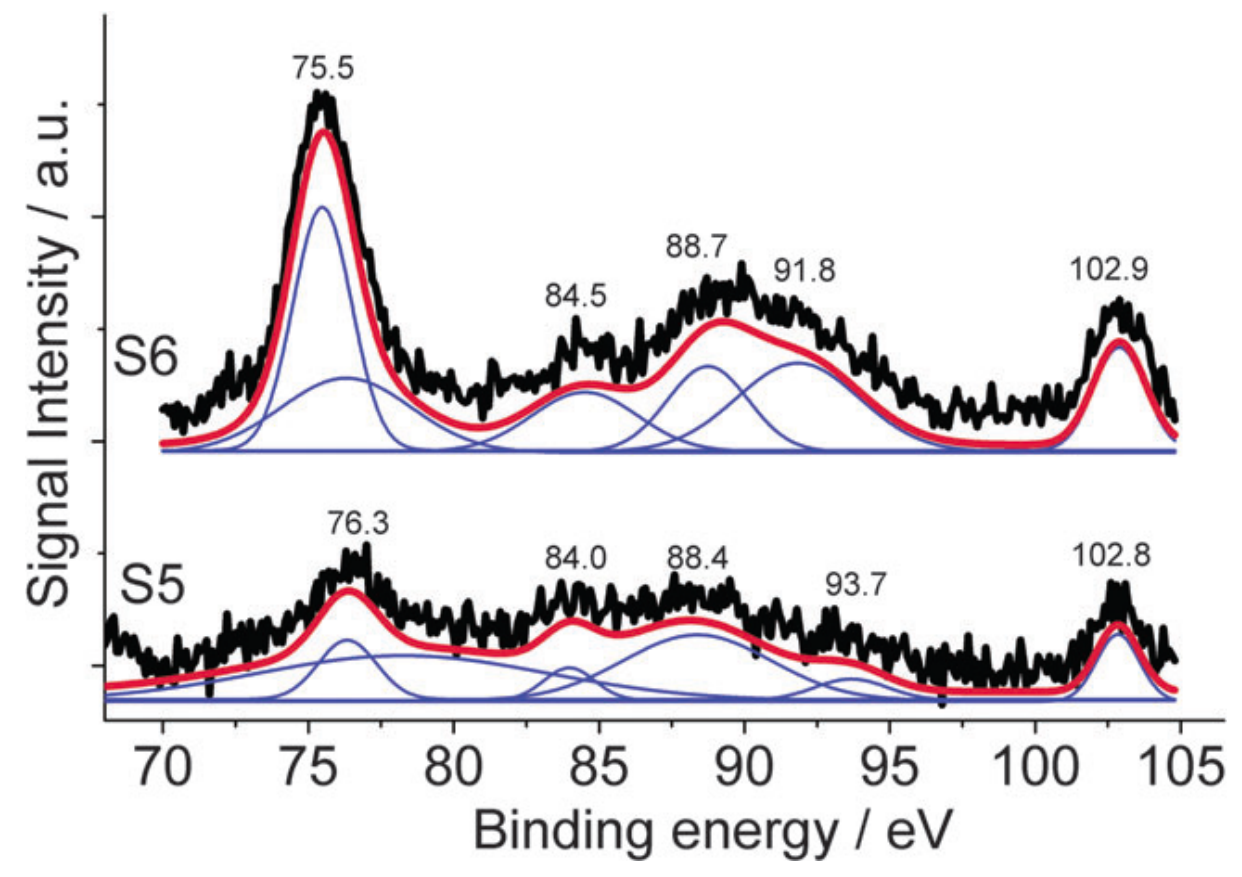

This color is reflected in UV-Visible optical spectroscopy by the presence of a weak absorption band centred at about $560 \mathrm{~nm}$ accompanied by the intense absorption due to P25 band-gap transition. 
The presence of increasing percentage of Pt is reflected in a gradual decrease in the surface plasmon band intensity but not in noticeable shifts in the $\lambda_{\max }$ position. Figure 3.3. presents the corresponding absorption optical spectra of some of the $\mathrm{Au}-\mathrm{Pt} / \mathrm{TiO}_{2}$ samples to illustrate the changes in the intensity and position of the bands depending on the Pt content.

Figure 3.3. Diffuse reflectance UV-Vis spectra (plotted as the Kubelka-Munk function of the reflectivity $\mathbf{R}$ ) for some selected samples. The inset shows a photograph of the $\mathrm{Au}-\mathrm{Pt} / \mathrm{TiO}_{2}$ samples ( $\mathrm{SX}, \mathrm{X}$ being the number indicated in the inset) used in this study.

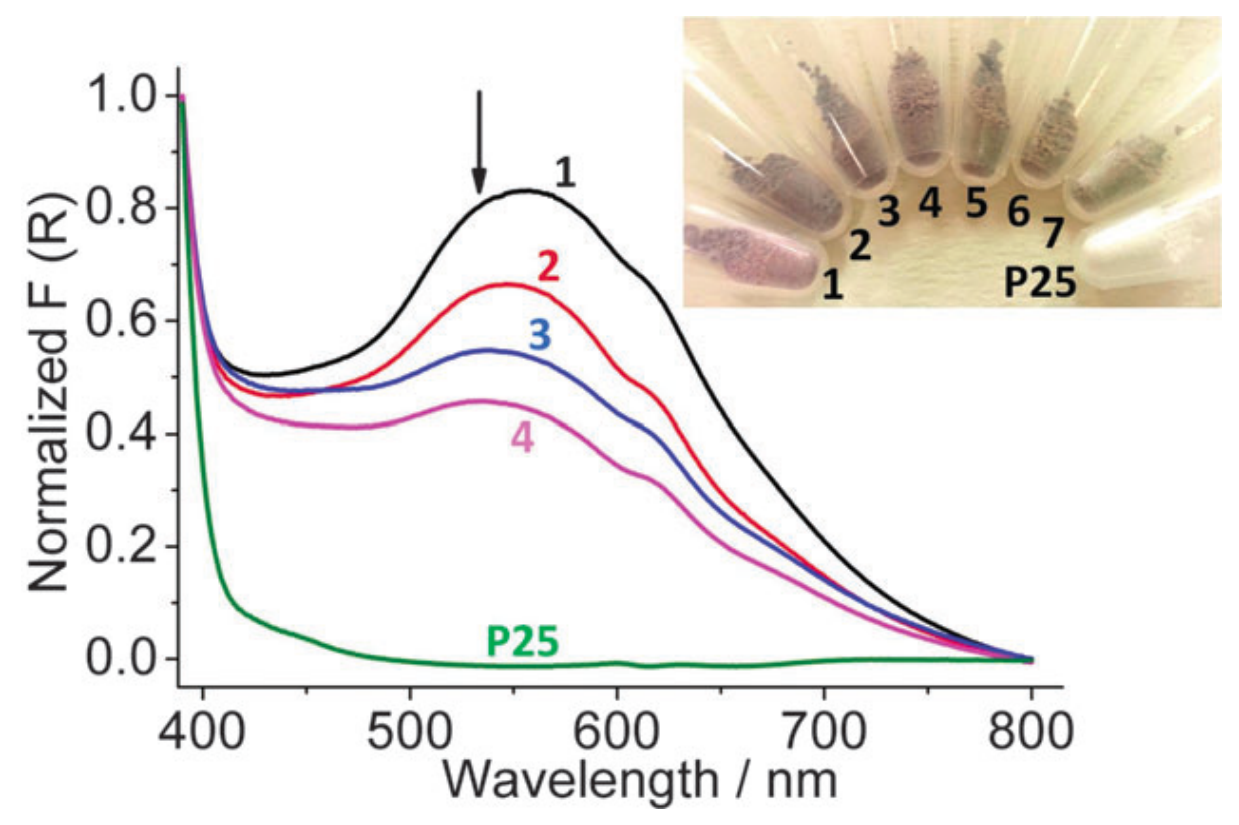

This figure also marks the $532 \mathrm{~nm}$ wavelength used for visible light excitation in the present study. Typically the $\lambda_{\max }$ of the surface plasmon band depends on the amount of other parameters and on the size of the nanoparticles. However, as we have commented when discussing Figure 3.1. presenting the histogram of particle size distribution, regardless of the Pt content, the size of the nanoparticles is very similar. Therefore, no difference in the position of the surface plasmon band should be expected. 


\subsubsection{Photocatalytic activity for hydrogen generation under visible light irradiation.}

To ensure that the photocatalytic hydrogen generation arises from visible light excitation and presumably from excitation of the $\mathrm{Au}-\mathrm{NP}$ surface plasmon band $\left(\lambda_{\max }\right.$ on $\left.\mathrm{TiO}_{2} 560 \mathrm{~nm}\right)$ all the experiments were carried out with the second harmonic output of a Nd-YAG laser emitting at $532 \mathrm{~nm}$ monochromatic light that is close to the maximum absorption for Au NPs. The experiments were carried out by suspending the corresponding photocatalyst in pure water or water containing $20 \%$ of methanol as the sacrificial electron donor under continuous stirring.

In preliminary experiments we performed the study of the influence of the amount of photocatalyst present in the system under laser irradiation on the hydrogen generation in order to optimize the evolution of hydrogen. The results are presented in Figure 3.4. As it could have been expected, the amount of hydrogen generated increases almost linearly with the amount of $\mathrm{Au} / \mathrm{TiO}_{2}$ photocatalyst up to a certain weight at which further increase even leads to a small decrease in the amount of hydrogen due to the fact that no more photons are absorbed for high weights of the photocatalyst and even light scattering and reflection are gaining importance in opaque dispersions. This optimal amount of photocatalyst was selected for further studies.

Also we initially studied the influence of the laser power on the amount of hydrogen evolved. The data for three different photocatalyst contents are presented in Figure 3.5. and show the same trend. No saturation of the hydrogen evolution reaching a plateau at the laser power increases is observed, indicating that, at the weight percentage of catalyst employed, the limiting factor is the number of photons entering into the system.

As it can be seen there, the curves of the amount of hydrogen vs. laser fluency are not linear but follow a quadratic dependence indicating that the process probably involves the simultaneous absorption of two photons under our irradiation conditions. 
Figure 3.4. Influence of the amount of S1 photocatalyst (plotted as weight percentage of S1 in the solution) on the hydrogen evolution after 1000 pulses upon $532 \mathrm{~nm}$ laser irradiation.

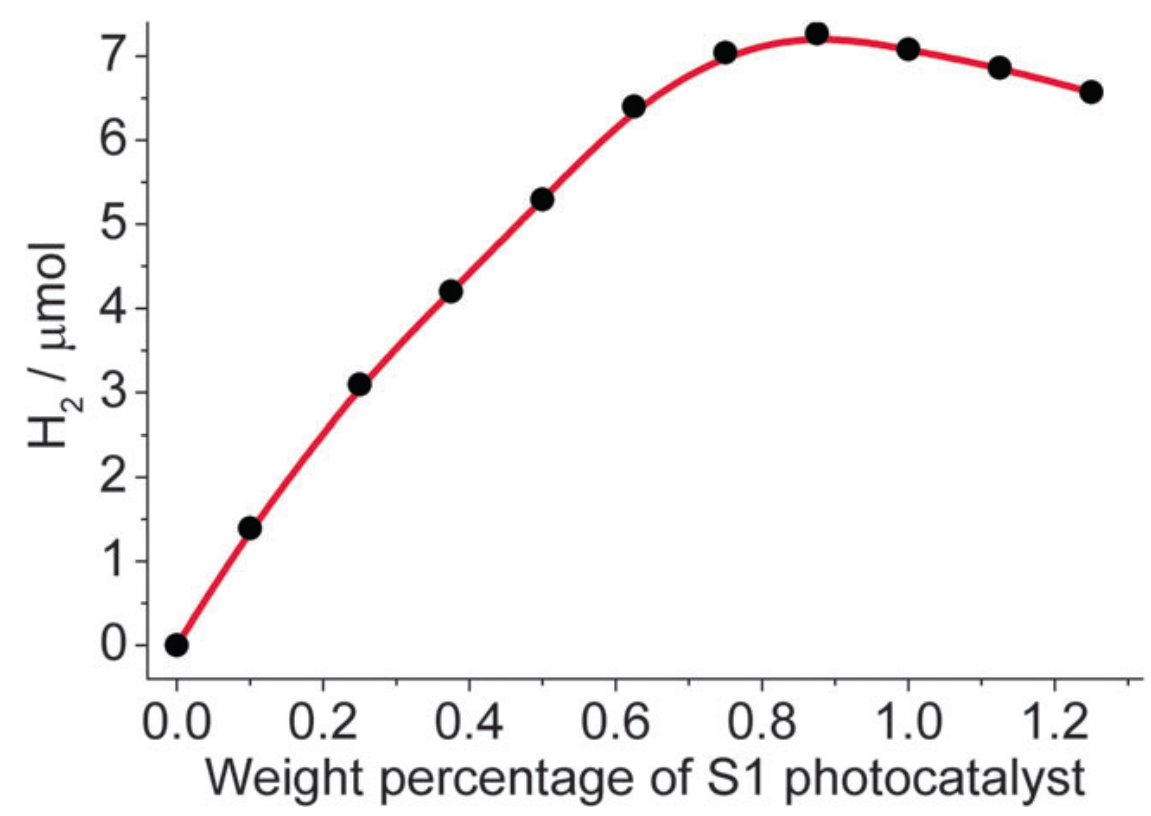

Figure 3.5. Influence of laser power on the hydrogen evolution upon $532 \mathrm{~nm}$ laser irradiation of 0.1 (black point), 0.25 (red point) or 0.5 (blue point) wt\% of S1 catalyst in a methanol-water solution (20:80). The continuous line corresponds to the best fitting of the experimental points to a quadratic equation.

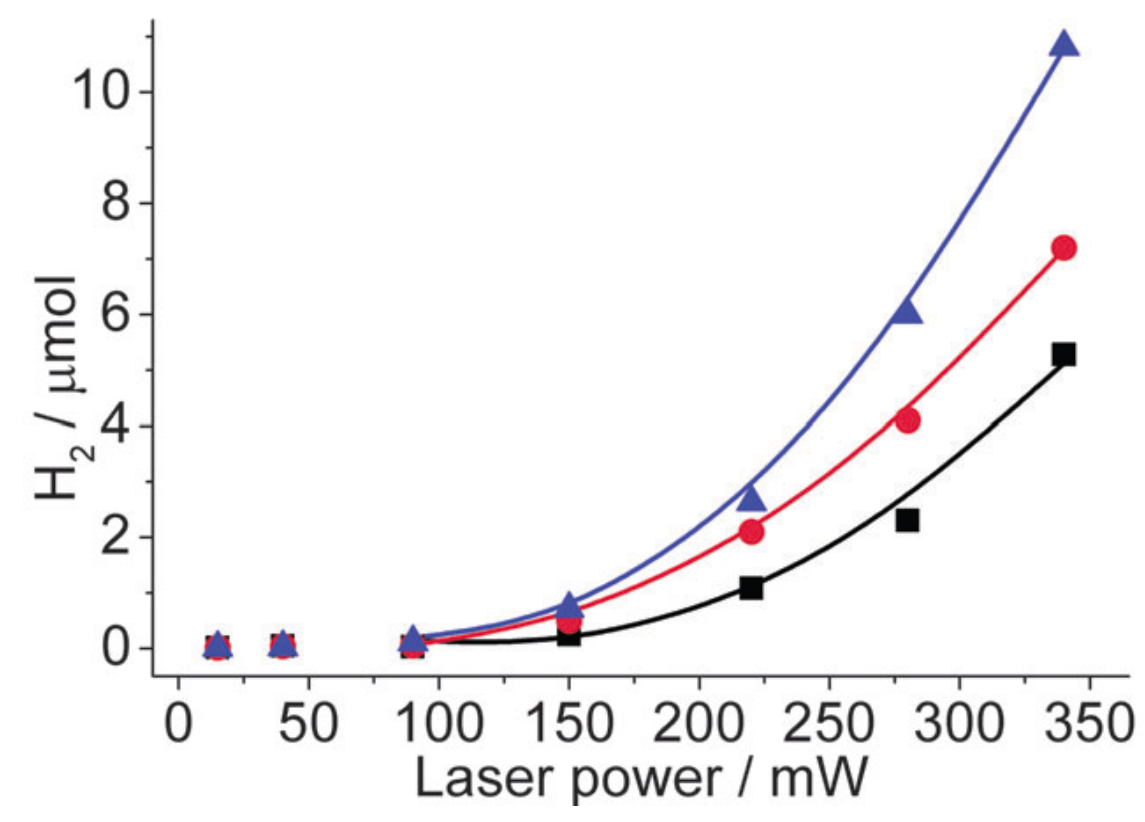


The possibility that the photocatalytic hydrogen generation under laser irradiation is a bi(multi)photonic process is not totally unreasonable considering that Au NPs may have a very large extinction coefficient (estimated to be $\sim 109 \mathrm{~mol}^{-1} \mathrm{~L} \mathrm{~cm}$ ) for $20 \mathrm{~nm}$ nanoparticles and the large photon fluency of the laser pulse. Although the exact power may vary from one experiment to other, the range of laser power was in the $300 \mathrm{~mW}$ region to ensure high ability for hydrogen generation. As it can be seen in Figure 3.5., under the experimental conditions used in this work $0.8 \mathrm{wt} \%$ and $300 \mathrm{~mW}$ ), the limiting parameter for hydrogen evolution is the light intensity, i.e. higher light fluencies will lead to higher $\mathrm{H}_{2}$ evolution. Contrasting results depending on the presence or absence of methanol were obtained. The results are shown in Figure 3.6. and 3.7. As expected in view of the well-established influence of methanol, the amount of hydrogen observed in the photolysis when this sacrificial agent was present was considerably larger than in its absence.

Preliminary blank controls using $\mathrm{TiO}_{2}$ subjected to the same experimental protocol as that followed for the preparation of the S1 sample show some hydrogen evolution (see Figure 3.6.) but was nine times lower than that measured for the $\mathrm{S} 1$ sample. This weak photocatalytic activity of $\mathrm{TiO}_{2}$ at $532 \mathrm{~nm}$ can be due to Raman laser scattering of the beam crossing the suspension that has a weak component at $415 \mathrm{~nm}$.

Importantly, no appreciable differences in the photocatalytic activity of the samples tested were observed in the presence of methanol.

Thus, the increasing percentage of Pt up to $50 \%$ in the nanoalloy does not play any influence on the efficiency. In contrast, in the absence of methanol, although the volume of hydrogen evolved was significantly lower than when methanol is present, remarkable differences were observed depending on the presence of Pt. Preliminary experiments using the parent $\mathrm{TiO}_{2}$ sample subjected to the same treatments as those followed to prepare S1 show that the hydrogen generation under these conditions was negligible compared to the S1 sample. 
Figure 3.6. Temporal hydrogen evolution upon $532 \mathrm{~nm}$ laser irradiation of $1 \mathrm{~mL}$ of a methanol aqueous solution (20 wt\%) containing $5 \mathrm{mg}$ of photocatalyst. The inset shows the efficiency of $\mathrm{H}_{2}$ generation in methanol-water for the photocatalysts tested for 1500 pulses. Colour and symbol code: S1 (black point), S2 (red point), S3 (green points), S4 (blue points), S5 (light blue points), S6 (violet points), S7 (orange points) and P25 (grey points).

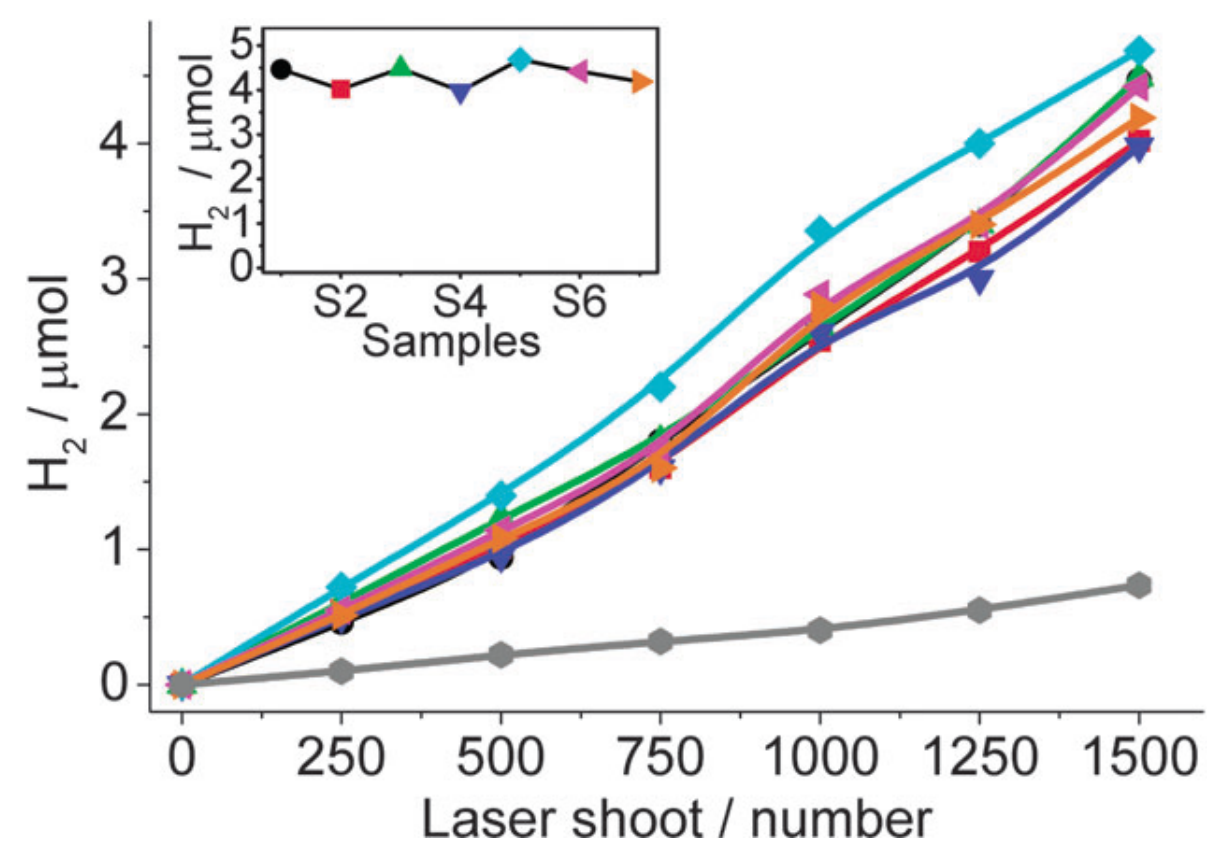

Concerning the influence of $\mathrm{Pt}$, the most efficient photocatalyst was the one in which no Pt was present $\left(\mathrm{Au} / \mathrm{TiO}_{2}\right)$, the presence of Pt producing a significant decrease in the photocatalytic activity (see Figure 3.7.). This negative effect is observed even at the lowest $\mathrm{Au}-\mathrm{Pt}$ atomic ratio tested. In fact, as inset in Figure 3.7. shows, the activity of all the samples containing some Pt was relatively constant. 
Figure 3.7. Temporal hydrogen evolution upon $532 \mathrm{~nm}$ laser irradiation of $1 \mathrm{~mL}$ of pure water containing $5 \mathrm{mg}$ of photocatalyst. Inset I shows the relative efficiency for $\mathrm{H}_{2}$ generation in water of the photocatalysts tested for 1500 pulses. Inset II shows, on the other hand, the oxygen evolution corresponding to S1 in the same experiment as the one in which $\mathrm{H}_{2}$ was measured. For Colour and symbol code see caption of Figure 7.6.

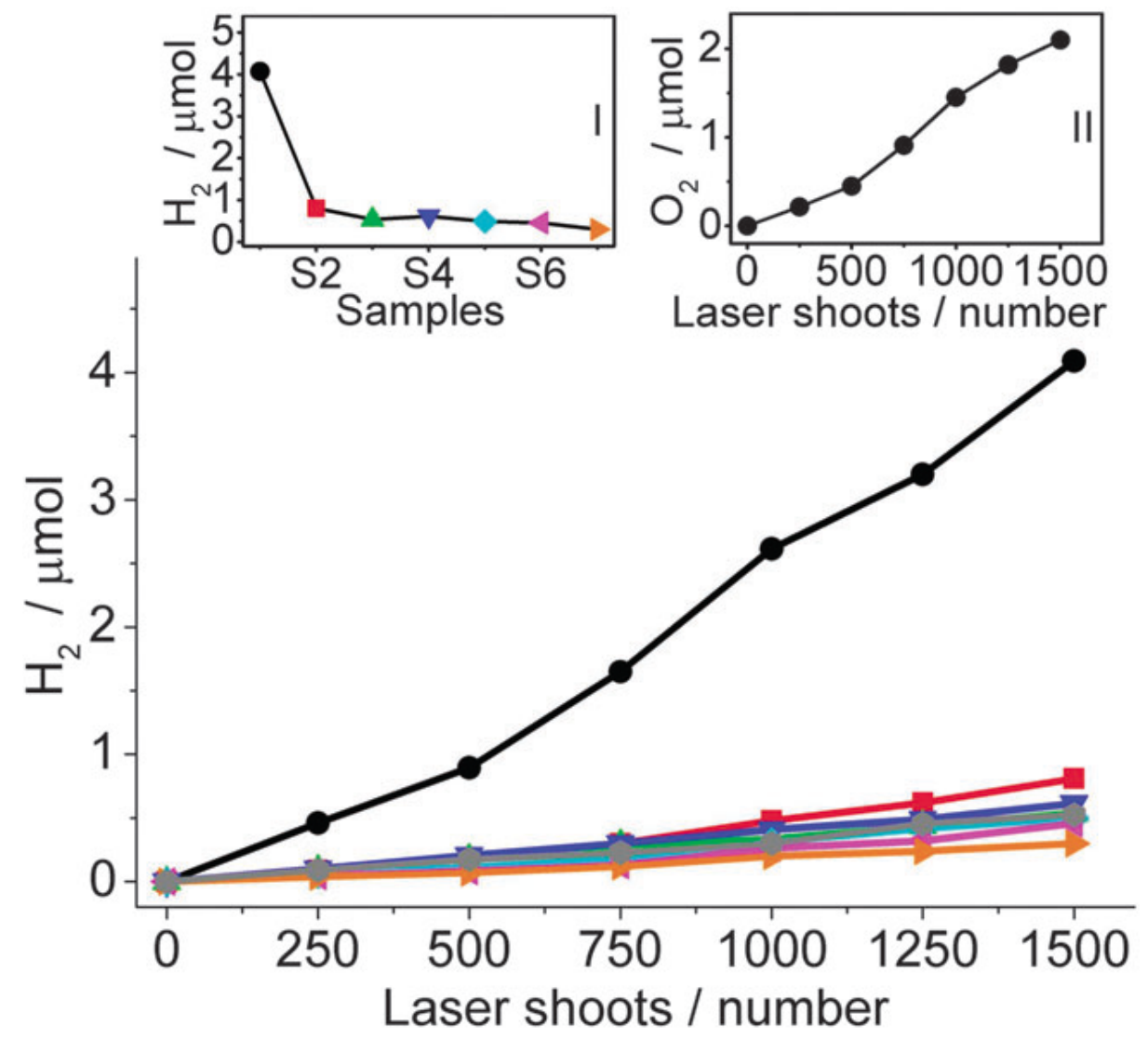

Considering the well known catalytic activity of Pt as a hydrogen evolving centre, these data seem to suggest that the rate determining step is not hydrogen evolution but other elementary processes taking place in the photocatalysis such as light absorption and electron injection of hot electrons from $\mathrm{Au}$ to the conduction band of the semiconductor or hole $\left(\mathrm{h}^{+}\right)$quenching. In this context, it is interesting to comment that in the photolysis of neat water, evolution of oxygen for the $\mathrm{Au} / \mathrm{TiO}_{2}$ photocatalyst was observed in the expected stoichiometrical amount (see inset II in Figure 3.7.).

Although experimental evidence of interfaced electron injection from excited $\mathrm{Au}$ NP to the conduction band of $\mathrm{TiO}_{2}$ is still missing, a mechanistic proposal based on charge separation with electrons located on the semiconductor and the positive 
hole on the Au NP has been suggested.[21] Scheme 3.1. illustrates the proposed mechanism for hydrogen generation upon visible light irradiation of the $\mathrm{Au} / \mathrm{TiO}_{2}$ photocatalyst. The key point is whether or not the oxidation potential of this $\mathrm{h}^{+}$site on $\mathrm{Au} \mathrm{NPs} \mathrm{is} \mathrm{still} \mathrm{sufficiently} \mathrm{high} \mathrm{to} \mathrm{effect} \mathrm{water} \mathrm{oxidation}\left(E_{\mathrm{ox}}^{\mathrm{o}}=1.26 \mathrm{~V}\right)$. Obviously, in the case that methanol is present, the formation of oxygen was not observed.

Scheme 3.1. Mechanistic proposal to explain the visible light response of $\mathrm{Au} / \mathrm{TiO}_{2}$ for the photocatalytic hydrogen generation from water. (a) photon absorption on plasmon band (b) creation of a charge separated state (c) reduction of $\mathrm{H}^{+}$to hydrogen accompanied by the oxidation of electron donor on gold nanoparticles.

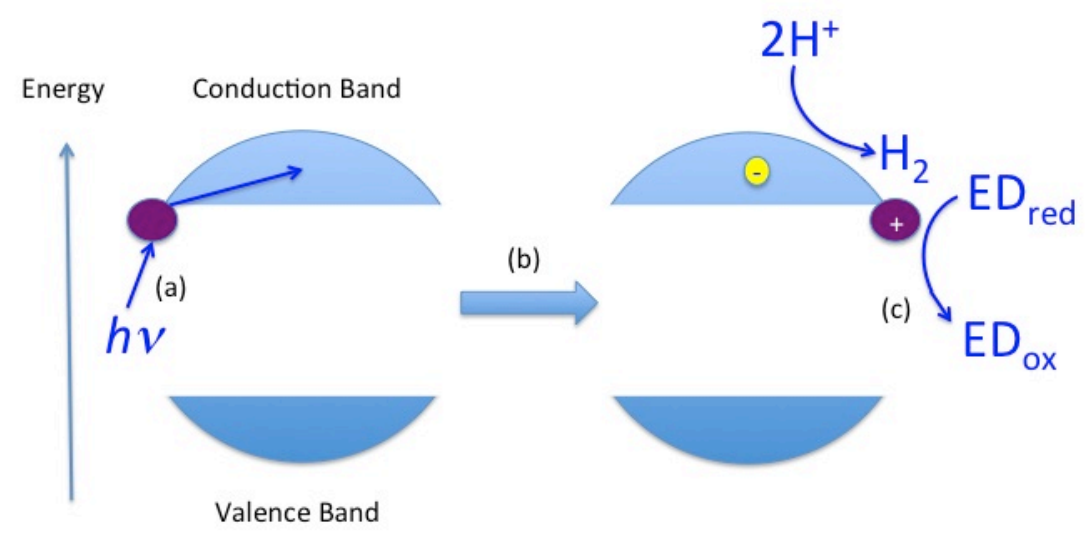

\subsubsection{Photocatalytic activity of $\mathrm{Au}-\mathrm{Pt} / \mathrm{TiO}_{2}$ under $\mathrm{UV}$ light or simulated sunlight.}

As commented earlier, the current challenge in photocatalytic hydrogen generation from solar light is to develop an efficient material under solar light illumination. While sunlight contains about $48 \%$ of energy in the visible region and only about $4 \%$ in the UV zone, it is frequently observed that the minor percentage of UV light is responsible for a large proportion of the photocatalytic activity, particularly for titania photocatalysts. Therefore, we were also interested in determining the relative activity of $\mathrm{Au}-\mathrm{Pt} / \mathrm{TiO}_{2}$ materials under $\mathrm{UV}$ light and simulated solar light. As we have already commented, precedents in the literature have already established that in the presence of UV radiations, Au-Pt nanoalloys are more efficient to enhance the photocatalytic activity of $\mathrm{TiO}_{2}$ than each of the noble metals independently. ${ }^{[27,28]}$ However, these studies were carried out at 
higher noble metal loadings ( 1 and $2 \mathrm{wt} \%$ compared to the $0.25 \mathrm{wt} \%$ reported here) and also for only one Au-Pt ratio (1:1). For this reason, our data have the interest to provide further information to establish the optimal proportion between $\mathrm{Pt}$ and $\mathrm{Au}$. In addition, the likely differences in the photocatalytic activities of the $\mathrm{Au}-\mathrm{Pt} / \mathrm{TiO}_{2}$ in the $\mathrm{UV}$ and in the visible range make difficult to predict the exact ranking of efficiencies under simulated solar light. In accordance with the previous report of Dal Santo and coworkers, ${ }^{[28]}$ under monochromatic $300 \mathrm{~nm}$ irradiation using methanol as the sacrificial electron donor, the less efficient sample was material S1 containing pure Au NPs. The efficiency under monochromatic UV irradiation increases with the presence and proportion of $\mathrm{Pt}$, the most efficient ones being those having the largest Pt content (photocatalysts S6 and S7). Figure 3.8 presents the hydrogen evolution vs. time under $300 \mathrm{~nm}$ irradiation. Worth noting is the fact that the ranking of activity under UV light is different to that previously measured under monochromatic visible light (see Figure 3.6.) and in general agreement with the precedents shows the higher photoactivity of Pt containing photocatalysts vs. those containing Au.

In view of this contrasting behavior depending on the irradiation wavelength, weither 532 or $300 \mathrm{~nm}$, it is of interest to determine the actual efficiency upon irradiation with polychromatic simulated sunlight. The results of hydrogen generation in a mixture of water-methanol (80:20 wt\%) using a solar simulator are shown in Figure 3.9.

Not surprisingly considering the different efficiency ranking between visible light (no influence of the proportion of Pt when methanol is present) and UV light (samples S6 and S7 having the highest Pt proportion are the most efficient), the results using simulated sun light are also different, the presence of a small proportion of Pt in the Au-Pt nanoalloys (samples S2 and S3) appearing now as the most efficient photocatalysts. 
Figure 3.8. Temporal hydrogen evolution upon monochromatic $300 \mathrm{~nm}$ irradiation. Colour and symbol code: S1 (black points), S2 (Red), S3 (green points), S4 (blue points), S5 (light blue points), S6 (violet points) and S7 (orange points).

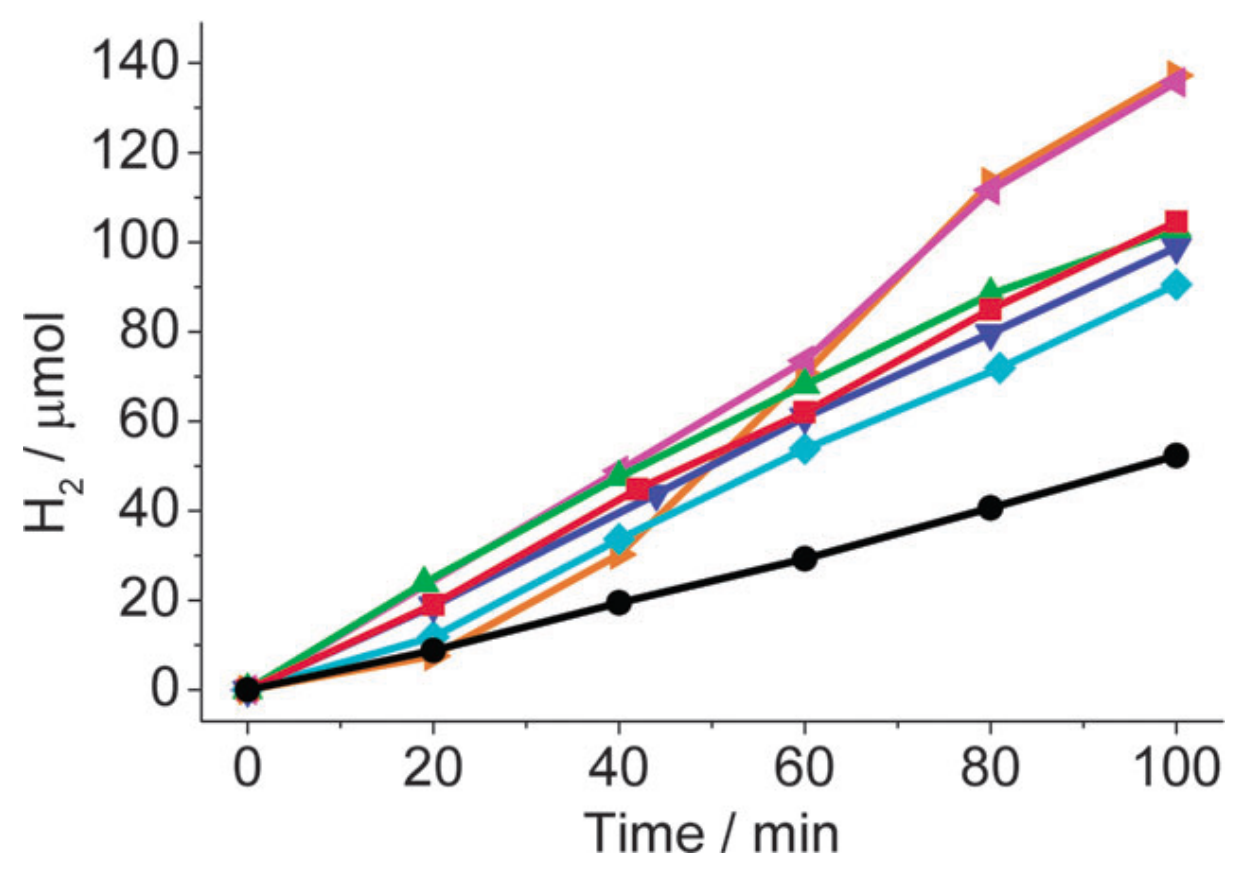

Figure 3.9. Temporal hydrogen evolution upon solar simulated irradiation. Color and symbol code: S1 (black points), S2 (red point), S3 (green poin), S4 (blue point), S5 (light blue point), S6 (violet point) and S7 (orange point).

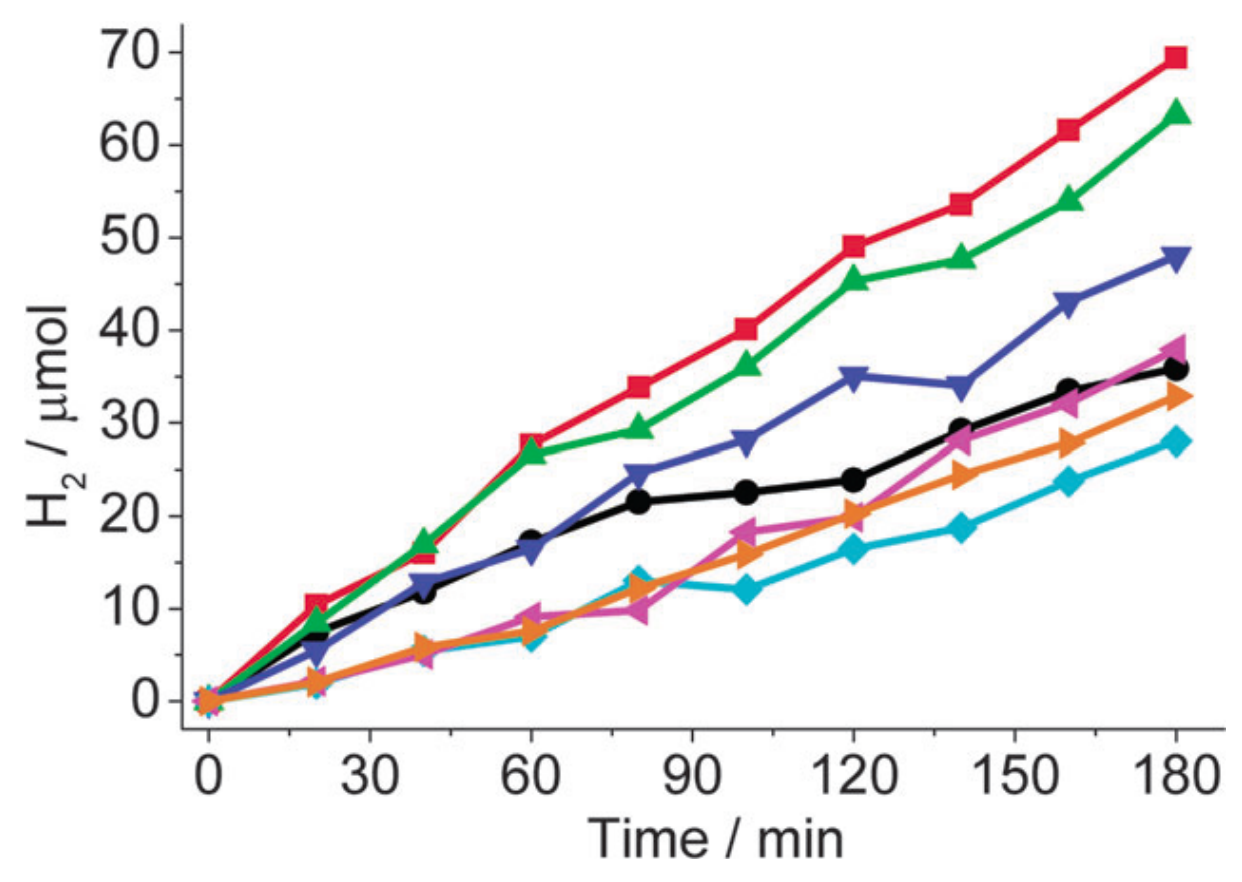




\subsubsection{Role of Pt in $\mathrm{Au}-\mathrm{Pt} / \mathrm{TiO}_{2}$.}

After having performed the previous experiments using visible, UV or polychromatic light as well as performing the experiments in the presence or absence of the sacrificial electron donor (methanol) the following general trends concerning the influence of Pt content on the nanoalloys can be deduced based on a rationalization that takes into account which is the light harvesting moiety and the influence of a fast hydrogen evolution.

When UV light is used for excitation, photons should be mainly absorbed by $\mathrm{TiO}_{2}$ and then the presence of a large percentage of $\mathrm{Pt}$ in the nanoalloy is beneficial because it accelerates hydrogen evolution (see Figure 3.8.). In contrast, when using exclusively visible light, photons should be absorbed by Au NPs and, in this case, the presence of Pt is detrimental when hydrogen evolution is not the controlling step (Figure 3.7.) or does not play any role when faster hydrogen evolution is promoted by some Pt in the cluster (Figure 3.6.). Under sunlight illumination that has a combination of UV and visible light, the situation is a balance between visible light absorption disfavoured by the presence of Pt and faster hydrogen evolution promoted by $\mathrm{Pt}$ reaching a compromise with an optimal Pt content in the nanoalloy (Figure 3.9.).

\subsection{Conclusions.}

By preparing a set of samples with the same overall metal loading (about 0.25 wt\%) but varying the proportion of $\mathrm{Pt}$, in the present chapter we have observed that in the absence of methanol as a sacrificial electron donor, a remarkable decrease in the photocatalytic activity at $532 \mathrm{~nm}$ is observed when Pt is present in the nanoparticles. In contrast, when methanol as a $\mathrm{h}^{+}$quencher is added to water, similar photocatalytic activity under $532 \mathrm{~nm}$ irradiation is observed for all the AuPt nanoalloys supported on P25. The order is different when the photocatalysts are excited at $300 \mathrm{~nm}$ in the presence of methanol, whereby the photocatalyst having the highest Pt content appears to be the most efficient one. When a solar simulator is used as an excitation source (continuous emission from 380 to 1000 $\mathrm{nm}$ ) then, an optimal percentage of Pt in the nanoalloy is observed. These results on one hand confirm the controversial visible light photocatalytic activity of $\mathrm{Au} / \mathrm{TiO}_{2}$ and on the other hand show the difference in the photocatalytic activity 
ranking depending on the excitation wavelength (monochromatic $532 \mathrm{~nm}$ or polychromatic UV-Vis irradiation). Controls comparing the photocatalytic activity of a $\mathrm{TiO}_{2}$ sample subjected to the same treatments used to prepare $\mathrm{Au} / \mathrm{TiO}_{2}$ show in fact some hydrogen evolution with the $532 \mathrm{~nm}$ laser but with considerably (at least nine times) lower efficiency than when $\mathrm{Au}$ was present. The reason for this contrasting behaviour appears to be direct excitation on the $\mathrm{TiO}_{2}$ semiconductor or photosensitization by the metal nanoparticles surface plasmon band. Further studies are still necessary to fully delineate the differences between UV and visible light irradiation in these systems and to understand and rationalize the origin of these differences. The results here show the potential of nanoalloys, allowing multiple metal combinations, to optimize the photocatalytic activity of $\mathrm{TiO}_{2}$.

\subsection{References.}

[1] G. Centi and S. Perathoner, ChemSusChem, 2010, 3, 195-208.

[2] G. W. Crabtree, M. S. Dresselhaus and M. V. Buchanan, Phys. Today, 2004, 57, $39-44$.

[3] S. Dunn, Int. J. Hydrogen Energy, 2002, 27, 235-264.

[4] D. Gust, T. A. Moore and A. L. Moore, Acc. Chem. Res., 2009, 42, 1890-1898.

[5] L. HammarstroÅNm and S. Hammes-Schiffer, Acc. Chem. Res., 2009, 42, 1859-1860.

[6] A. Haryanto, S. Fernando, N. Murali and S. Adhikari, Energy Fuels, 2005, 19, 2098-2106.

[7] N. S. Lewis and D. G. Nocera, Proc. Natl. Acad. Sci. U. S. A., 2006, 103, 1572915735.

[8] V. Balzani, A. Credi and M. Venturi, ChemSusChem, 2008, 1, 26-58.

[9] A. J. Bard and M. A. Fox, Acc. Chem. Res., 1995, 28, 141-145.

[10] C. C. Chen, P. X. Lei, H. W. Ji, W. H. Ma, J. C. Zhao, H. Hidaka and N. Serpone, Environ. Sci. Technol., 2004, 38, 329-337.

[11] X. Chen and S. S. Mao, Chem. Rev., 2007, 107, 2891-2959.

[12] A. Fujishima, X. Zhang and D. A. Tryk, Surf. Sci. Rep., 2008, 63, 515-582.

[13] K. Maeda and K. Domen, J. Phys. Chem. Lett., 2006, 1, 2655-2661.

[14] C. Burda, Y. B. Lou, X. B. Chen, A. C. S. Samia, J. Stout and J. L. Gole, Nano Lett., 2003, 3, 1049-1051. 
[15] J. M. Herrmann, Catal. Today, 1999, 53, 115-129.

[16] M. I. Litter and J. A. Navio, J. Photochem. Photobiol. A, 1996, 98, 171-181.

[17] A. Primo, A. Corma and H. Garcia, Phys. Chem. Chem. Phys., 2011, 13, 886910.

[18] A. Primo, T. Marino, A. Corma, R. Molinari and H. Garcia, J. Am. Chem. Soc., 2011, 133, 6930-6933.

[19] S. Sakthivel, M. Janczarek and H. Kisch, J. Phys. Chem. B, 2004, 108, 1938419387.

[20] N. Serpone, D. Lawless and R. Terzian, Solar Energy, 1992, 49, 221-234.

[21] C. G. Silva, R. Juarez, T. Marino, R. Molinari and H. Garcia, J. Am. Chem. Soc., 2011, 133, 595-602.

[22] H. J. Yan, J. H. Yang, G. J. Ma, G. P. Wu, X. Zong, Z. B. Lei, J. Y. Shi and C. Li, J. Catal., 2009, 266, 165-168.

[23] J. C. Yu, W. K. Ho, J. G. Yu, H. Yip, P. K. Wong and J. C. Zhao, Environ. Sci. Technol., 2005, 39, 1175-1179.

[24] X. Zong, H. J. Yan, G. P. Wu, G. J. Ma, F. Y. Wen, L. Wang and C. Li, J. Am. Chem. Soc., 2008, 130, 7176. Q7

[25] A. Corma, P. Serna, P. Concepcion and J. J. Calvino, J. Am. Chem. Soc., 2008, $130,8748-8753$.

[26] T. Mallat and A. Baiker, Chem. Rev., 2004, 104, 3037-3058.

[27] A. Gallo, M. Marelli, R. Psaro, V. Gombac, T. Montini, P. Fornasiero, R. Pievo and V. Dal Santo, Green Chem., 2012, 14, 330-333.

[28] A. Gallo, T. Montini, M. Marelli, A. Minguzzi, V. Gombac, R. Psaro, P. Fornasiero and V. Dal Santo, ChemSusChem, 2012, 5, 1800-1811.

[29] G. R. Bamwenda, S. Tsubota, T. Kobayashi and M. Haruta, J. Photochem. Photobiol., A, 1994, 77, 59-67. 
Chapter 4

Photocatalytic hydrogen generation from water-methanol mixtures using "black" anatase obtained by annealing of titanate nanotubes. 
Chapter 4 - Photocatalytic hydrogen generation from water-methanol mixtures using “black” anatase obtained by annealing of titanate nanotubes.

\subsection{Introduction.}

In the context of photocatalytic hydrogen generation from water-methanol mixtures there is a continuous interest in the development of more efficient photocatalysts.[1-8] $\mathrm{TiO}_{2}$-based materials have been the most widely used photocatalysts for this reaction. ${ }^{[1-3,7,8]}$ It had been found that certain parameters including the crystal phase, ${ }^{[9-11]}$ the presence of dopant elements, ${ }^{[12-17]}$ the morphology ${ }^{[18,19]}$ and structure ${ }^{[19]}$ of the material exert a strong influence of the resulting photocatalytic activity. Related to our work are several reports in where titanium dioxide had been submitted to annealing under hydrogen atmosphere to obtain a "black" $\mathrm{TiO}_{2}$ nanoparticles.[20] It was found that black titania nanoparticles (loaded with $0.6 \mathrm{wt} \% \mathrm{Pt}$ ) exhibit enhanced photocatalytic activity for hydrogen generation from water.[20] It has been proposed that the black $\mathrm{TiO}_{2}$ sample exhibits an enhanced photocatalytic activity due to the increased light absorption in the visible region. Characterization of black titania suggests that thermal treatment with hydrogen produces the appearance of a black solid due to the partial amorphization of the outermost layers of the $\mathrm{TiO}_{2}$ nanoparticle.[20] Formation of "black" $\mathrm{TiO}_{2}$ nanotubes displaying visible-light $(\lambda>450 \mathrm{~nm})$ photocatalytic activity for the oxidation of acetaldehyde has also been reported.[21] In this precedent, it was found that the black colour of titania nanotubes arises from chromium contamination from the stain steel reactor in which the thermal annealing was performed.[21] Titania nanotubes annealed at various temperatures and exhibiting visible light absorption have also been used as photocatalyst for the degradation of dyes. ${ }^{[22]}$ Continuing with this research aimed at developing more efficient titania photocatalysts by submitting $\mathrm{TiO}_{2}$ to hydrogen annealing, in the present manuscript we report that titania nanoparticles formed by thermal treatment of titania nanotubes under hydrogen exhibits an enhanced photocatalytic activity for hydrogen generation from water-methanol mixture that is even higher than the activity of an analogous sample of "black" titania obtained from the direct treatment of $\mathrm{TiO}_{2}$ nanoparticles by hydrogen. 


\subsection{Results and discussion.}

The titania samples under study were prepared starting from titanate nanotube obtained from $\mathrm{TiO}_{2}$ digestion under strong basic condition following reported procedures. ${ }^{[23]}$ Scheme 4.1 summarizes the preparation of the samples under study. Starting from anatase titania nanoparticles (20 nm, sample M0), the solid was submitted to hydrothermal digestion with highly concentrated $10 \mathrm{M} \mathrm{NaOH}$ aqueous solution in a Teflon-lined autoclave at $125{ }^{\circ} \mathrm{C}$ for 4 days. This crystallization time ensures high homogeneity of the sample and a better crystallinity. ${ }^{[21,23]}$ After the prolonged treatment under strong basic conditions, the solid was collected by filtration and washed with $\mathrm{HCl}$ at $\mathrm{pH} 1.5$ and finally washed with water until neutral $\mathrm{pH}$ and dried in an oven at $120^{\circ} \mathrm{C}$ for 2 days (sample M1). After this treatment, the resulting solid material M1 exhibits a light brown colour. In agreement with the precedents reported in the literature, ${ }^{[23]}$ these treatments lead to the formation of titanate nanotubes from $\mathrm{TiO}_{2}$ nanoparticles and we obtained a sample with the same morphology at the nanometric scale as reported by Tsai and Theng. ${ }^{[23]}$ We noticed, however, that the original report did not mention any colour in the resulting titanate that in reality are brown in appearance. Figure 4.1. shows a representative transmission electron microscopy image of the titanate nanotubes M1 obtained by strong basic hydrothermal treatment. As it can be seen in this Figure, a characteristic morphology of the crystals defined by a high aspect ratio, with length of several hundreds of nanometres and width about ten nanometres, was obtained for the light brown sample. 
Scheme 4.1. Preparation procedure of the samples under study

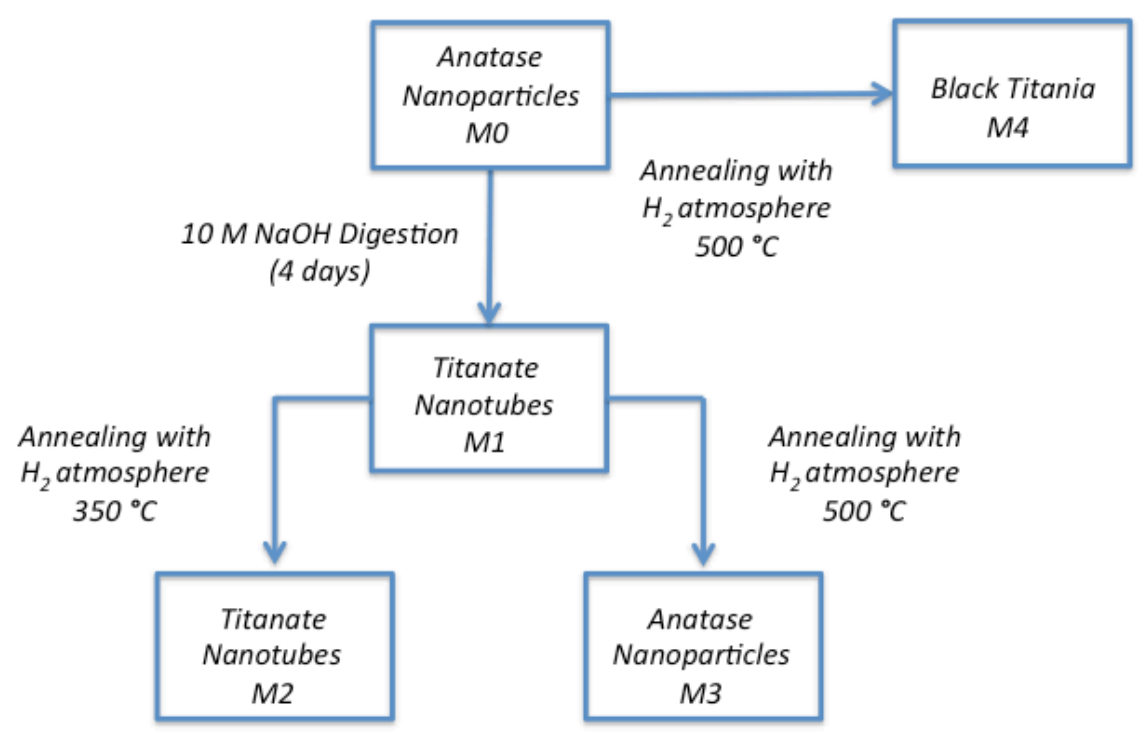

Figure 4.1. TEM images recorded for M1 (inset A), M2 (inset B), M4 (inset C). STEM images recorded for M3 (inset D and E).
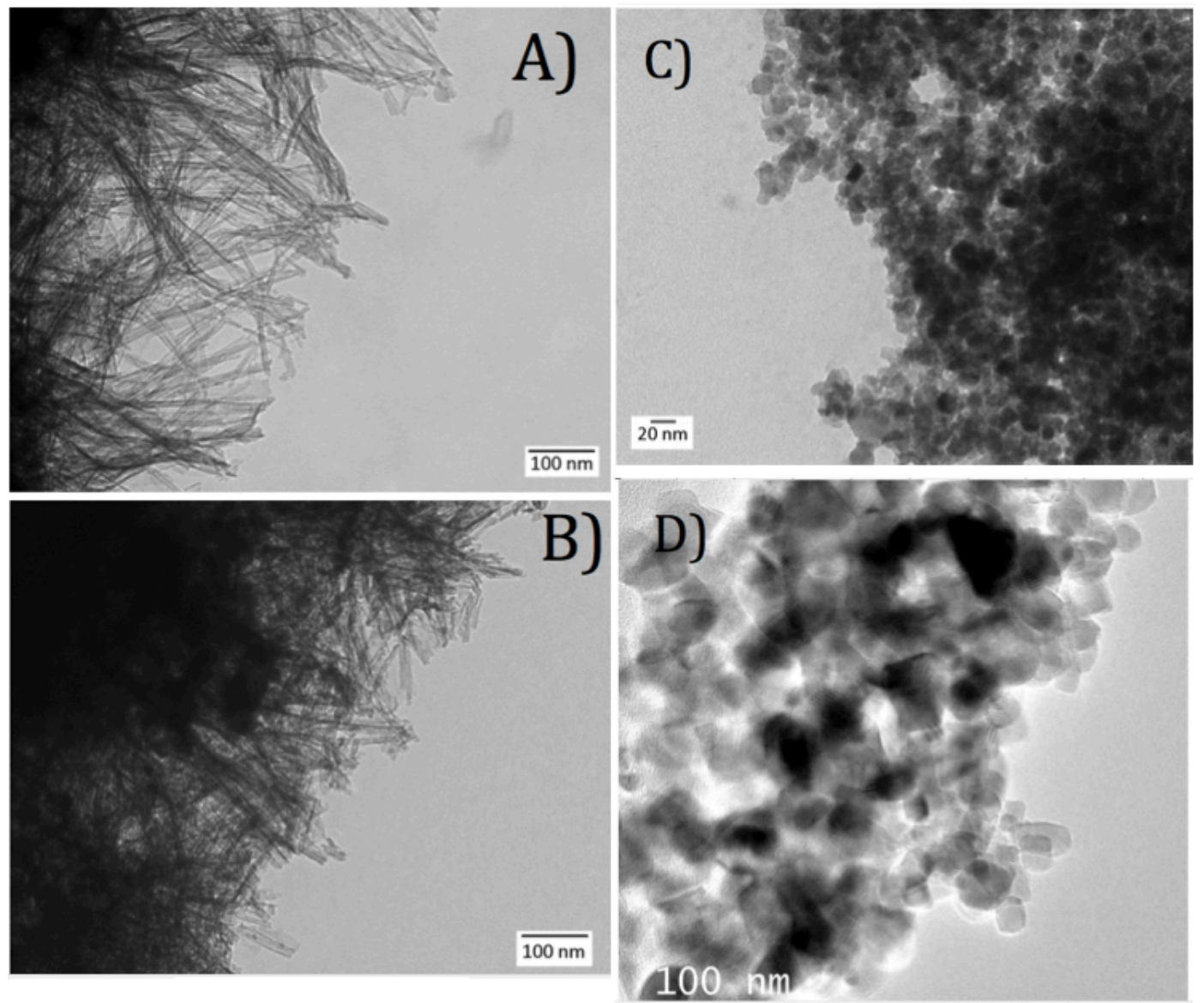
As indicated in Scheme 4.1. the purpose of our work was to submit this sample of titanate nanotubes to annealing under hydrogen at increasing temperatures in order to obtain "black" titanium materials, due to the amorphization of the external surface with creation of oxygen vacancies on the external surface. ${ }^{[24]}$ For this reason the sample after digestion with $\mathrm{NaOH}$ (M1) was annealed under hydrogen at $350(\mathrm{M} 2)$ or $500{ }^{\circ} \mathrm{C}(\mathrm{M} 3)$. As can be seen in Figure 4.1., this reductive annealing maintains (in the case of sample M2) or changes (in case of M3) the crystal phase and morphology of the samples. In any case, the intensity of the colour of the solid always increased after hydrogen annealing. The inset of Figure 4.2. shows a photograph of the resulting M1-3 samples. In accordance with the appearance of more (M3) o less intense (M1 and M2) colour after this treatment, Figure 4.2. shows the diffuse reflectance UV-Vis spectra of the M1-3 samples in where the presence of an absorption in the visible region can be observed. This broad continuous absorption expands all wavelength range decreasing in intensity toward the red, this explaining the brown/black visual appearance of the M1-M3 samples.

Figure 4.2. Diffuse reflectance UV-Vis absorption spectra [plotted as the KubelkaMunk function of the remittance [R]] of the set of titanium samples under study. The top inset shows a magnification of the $400-500 \mathrm{~nm}$ region. The bottom inset shows a photograph of the samples (left to right M0, M1, M2, M3 and M4).

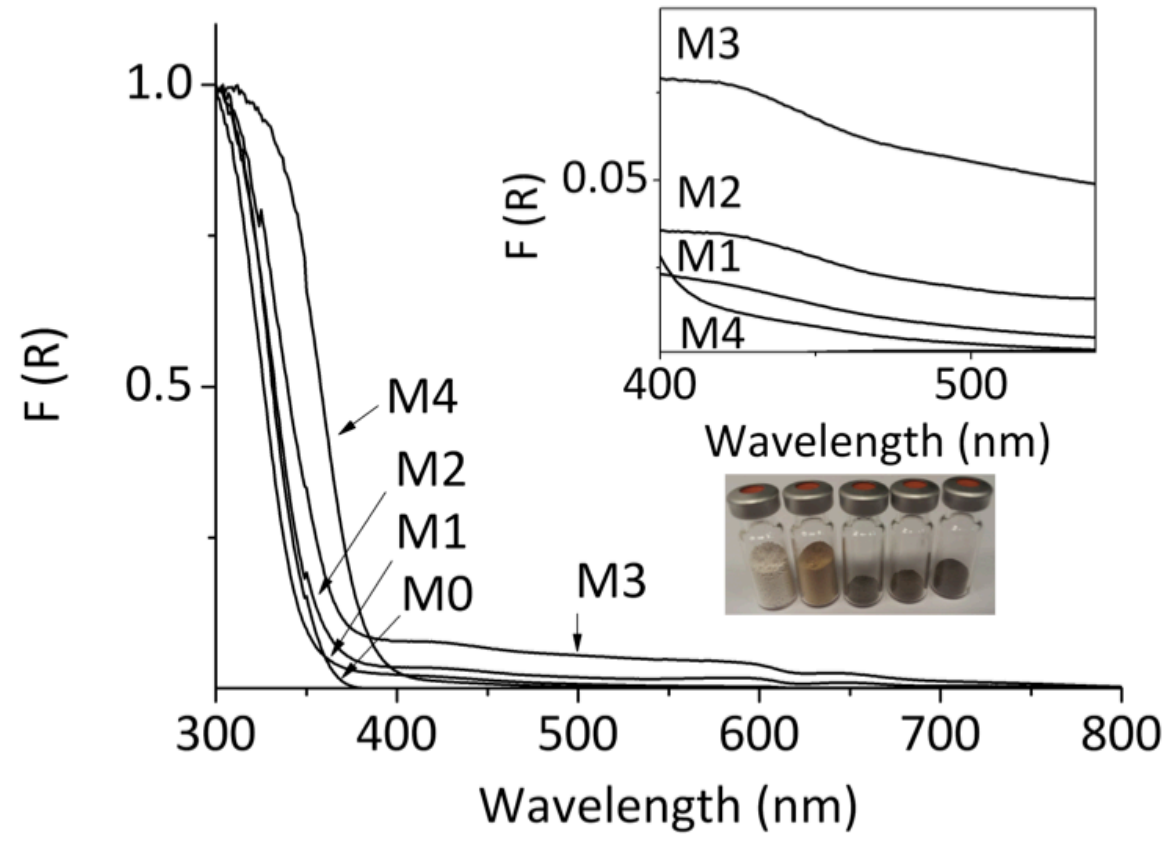


The crystal phase and crystallinity of the samples was addressed by XRD. Figure 4.4. shows the XRD patterns of the M1-3 samples in comparison with the XRD of the starting titania sample (M0). It can be seen that while the starting material M0 is highly crystalline, $\mathrm{NaOH}$ digestion decreases significantly the crystallinity of $\mathrm{M} 1$ leading to peaks in XRD of weak intensity, in agreement with the reports in the literature for titanate samples. ${ }^{[23]}$ The characteristic XRD of M1 did essentially not change for $\mathrm{M} 2$, but when the hydrogen annealing was carried out at $500{ }^{\circ} \mathrm{C}$, then, XRD shows that the crystallinity of M3 sample increases and is very similar to that of the parent $\mathrm{TiO}_{2}(\mathrm{M} 0)$ corresponding to the anatase phase. This increase in the crystallinity from M2 to M3 correlates well with the change in the morphology of the particles from M2 to M3 as revealed by TEM and indicates that the visual colour is independent of the crystal phase. For the sake of comparison our set of sample also includes an additional $\mathrm{TiO}_{2}$ material that was produced by hydrogen annealing of $\mathrm{M} 0$ without $\mathrm{NaOH}$ digestion (M4, see Scheme 4.1.). As commented in the introduction, it had been reported that "black" titania can be obtained by prolonged hydrogen treatment of $\mathrm{TiO}_{2}$ nanoparticles. ${ }^{[20]}$ We have been able to reproduce the preparation of "black" titania (M4). The TEM images, the diffuse reflectance UV-Vis spectra and XRD patterns of this M4 sample are also included in Figures 4.1., 4.2., and 4.4., respectively.

The valence state of titanium atoms in the samples was determined by XPS. Clear changes were observed in the position of the Ti $2 p$ and 01 s in XPS peaks for titanate samples M1 and M2 with respect to M3. Figure 4.3. shows the XPS peaks corresponding to Ti $2 \mathrm{p}$ and 0 1s for samples M1 and M3, where no components attributable to $\mathrm{Ti}^{3+}$ are observed.

In the case of the oxygen peaks for sample M3, it has been possible to make the deconvolution of the experimental peaks in two components, the mayor one at 530. eV (58\% of the signal) appears at the typical binding energy of anatase, while the minor one (42\%) at $532.8 \mathrm{eV}$ is coincident with the binding energy value for sample M1. 
Figure 4.3. XPS analysis of the samples M1 and M3 A) XPS analysis of Ti 2p for samples M1 and M3 B) XPS analysis of oxygen of samples M1 and M3
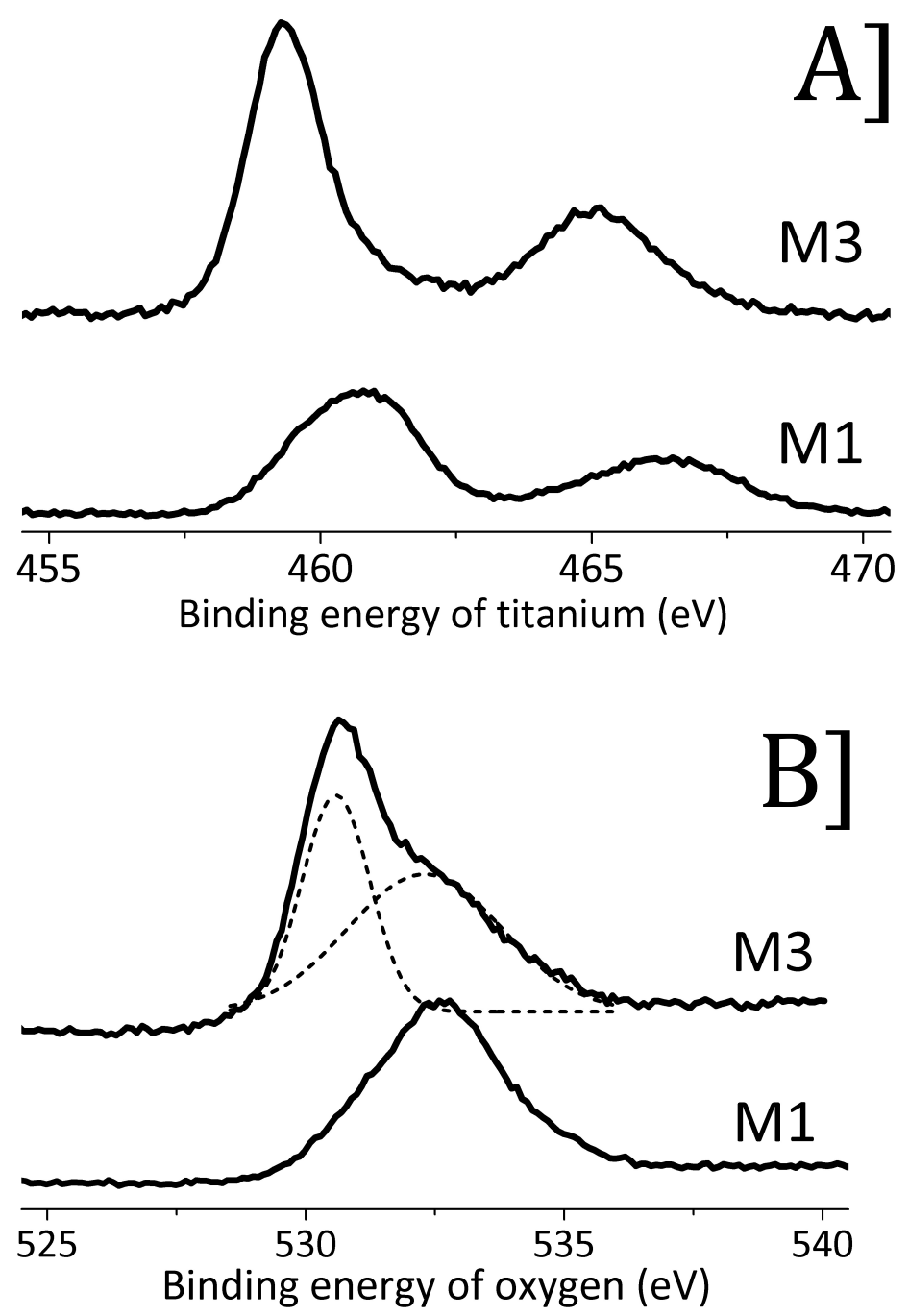

The chemical treatment and the crystal phase change taking place during annealing have also consequence in the BET surface area of the materials (see Table 4.1.). The change from $M 0$ to $M 1$ results in a remarkable increase of over six times in the surface area that basically remain when the $\mathrm{H}_{2}$ treatment is performed to $350{ }^{\circ} \mathrm{C}(\mathrm{M} 2)$ but undergoes a significant decrease when $\mathrm{M} 1$ is annealed at $500{ }^{\circ} \mathrm{C}$ in the preparation of M3.

As summary, Table 4.1. lists the titania samples under study in the present work, and the preparation conditions as well as colour, crystal phase and BET surface area. 
Table 4.1. Code, morphology, preparation procedure, relevant textural and photocatalytic data of the samples under study

\begin{tabular}{|l|l|l|l|l|l|}
\hline Sample & $\begin{array}{l}\text { Crystal } \\
\text { Phase }\end{array}$ & Preparation method & $\begin{array}{l}\text { BET Area } \\
{\left[\mathrm{m}^{2} / \mathrm{g}\right]}\end{array}$ & $\begin{array}{l}\text { H2 Production ( } \mu \text { mol) } \\
\text { Solar Simulator (a) Laser 532 } \mathrm{nm}^{(\mathrm{b})}\end{array}$ \\
\hline M0 & $\begin{array}{l}\text { Anatase } \\
\text { Particle }\end{array}$ & Commercial sample & 55.0 & 3.10 & 0.02 \\
\hline M1 & $\begin{array}{l}\text { Titanate } \\
\text { Nanotubes }\end{array}$ & NaOH digestion & 366.5 & 0.96 & 0.10 \\
\hline M2 & $\begin{array}{l}\text { Titanate } \\
\text { Nanotubes }\end{array}$ & $\begin{array}{l}\text { NaOH digestion/ } \mathrm{H}_{2} \\
\text { treatment at 350 }{ }^{\circ} \mathrm{C}\end{array}$ & 351.6 & 0.65 & 0.39 \\
\hline M3 & $\begin{array}{l}\text { Anatase } \\
\text { Particles }\end{array}$ & $\begin{array}{l}\text { NaOH digestion/ } \mathrm{H}_{2} \\
\text { treatment at } 500^{\circ} \mathrm{C}\end{array}$ & 166.1 & 3.87 & 0.54 \\
\hline M4 & $\begin{array}{l}\text { Anatase } \\
\text { Particles }\end{array}$ & $\begin{array}{l}\text { M0 treated with } \mathrm{H}_{2} \\
\text { at } 500^{\circ} \mathrm{C}\end{array}$ & 36.5 & 2.96 & 0.07 \\
\hline
\end{tabular}

(a) $\mu \mathrm{mol}$ at $3 \mathrm{~h}$ irradiation (b) $\mu \mathrm{mol}$ at 300 shots

Figure 4.4. XRD of the titanium-containing samples under study.

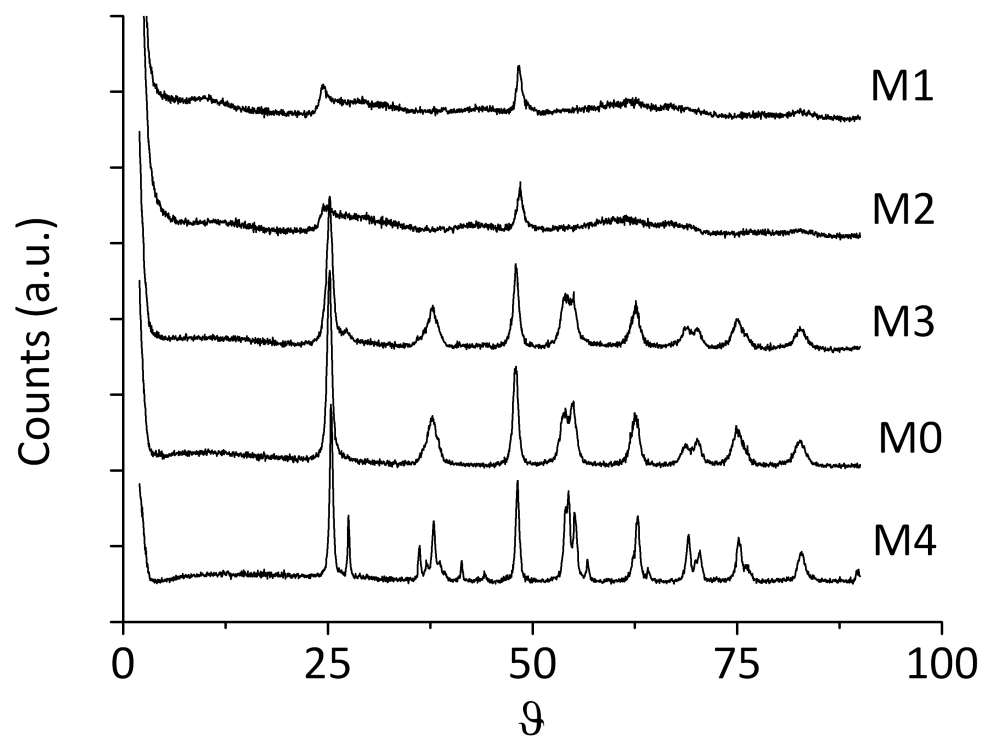




\subsubsection{Photocatalytic hydrogen production.}

Samples listed in Table 4.1 were tested with respect to their activity for hydrogen production from water-methanol [3:1] mixtures under monochromatic, either visible or UV light excitation at $532 \mathrm{~nm}$ and 355, and under polychromatic, lowerintensity irradiation using simulated sunlight. The results obtained are presented in Figures 4.5. - 4.7. for $532 \mathrm{~nm}, 355 \mathrm{~nm}$ or simulated solar light, respectively. Figure 4.8., on the other hand, provides the photoaction spectrum in comparison with the absorption spectrum for the most active sample M3.

Figure 4.5. Photocatalytic hydrogen production of M0-M4 samples under $532 \mathrm{~mm}$ irradiation. Reaction conditions: Water-methanol [3:1] mixture $2 \mathrm{ml}$, photocatalyst

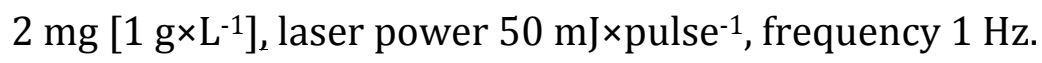

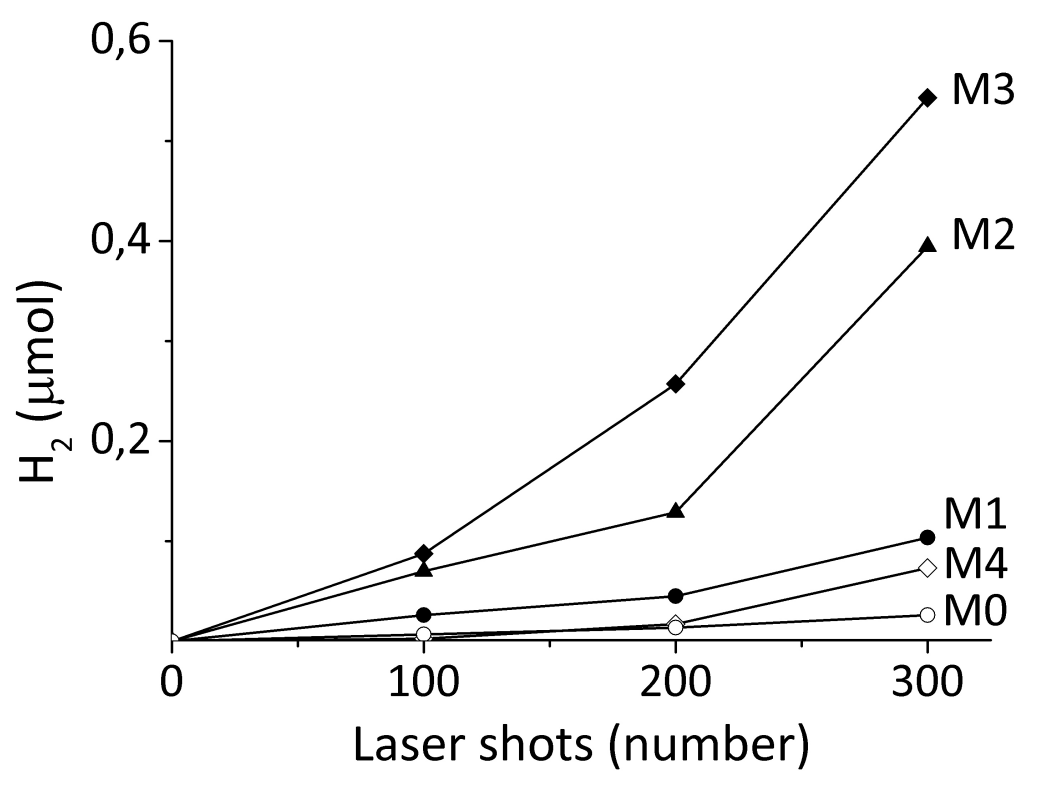


Figure 4.6. Photocatalytic hydrogen production of M0-M4 samples under $355 \mathrm{~mm}$ irradiation. Reaction conditions: Water-methanol [3:1] mixture $2 \mathrm{ml}$, photocatalyst $2 \mathrm{mg}\left[1{\left.\mathrm{~g} \times \mathrm{L}^{-1}\right] \text {, laser power } 50 \mathrm{~mJ} \times \text { pulse }}^{-1}\right.$, frequency $1 \mathrm{~Hz}$.

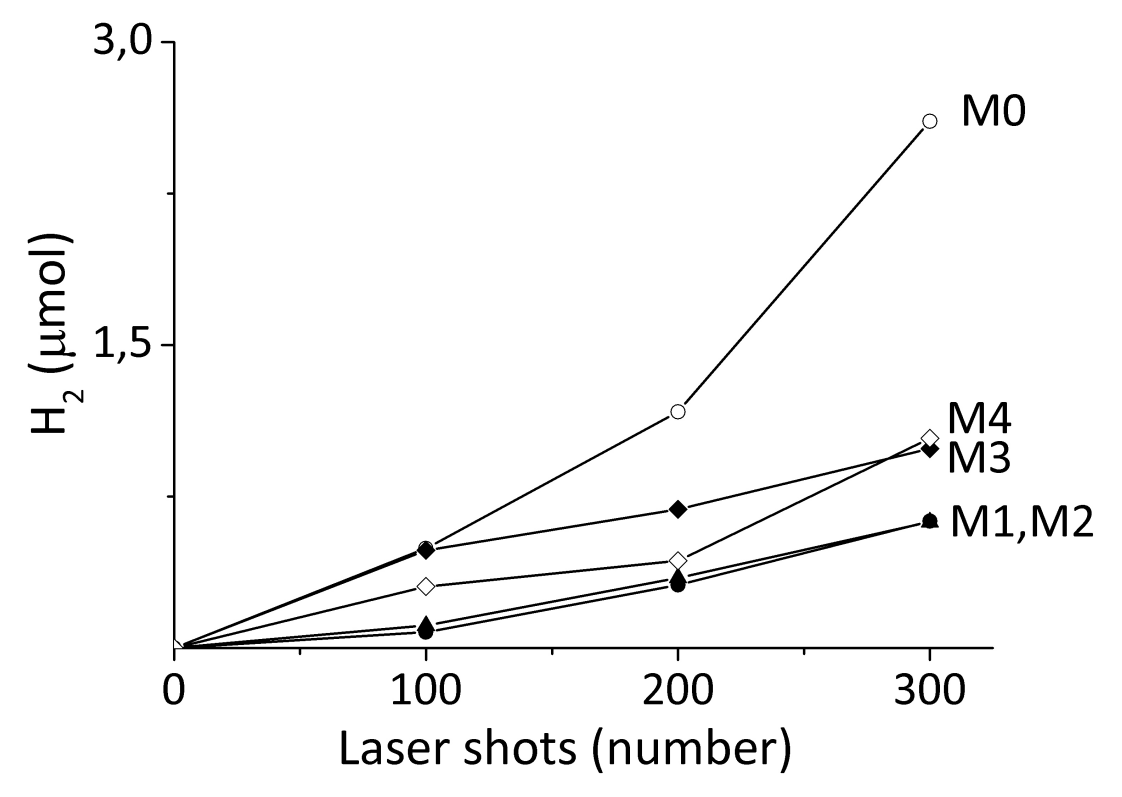

Figure 4.7. Photocatalytic hydrogen production from water-methanol [3:1] mixtures using sample M0-M4 as photocatalyst under simulated sunlight irradiation. Reaction condition: Water-methanol [3:1] mixtures $25 \mathrm{~mL}$, catalyst concentration $1 \mathrm{~g} \times \mathrm{L}^{-1}$.

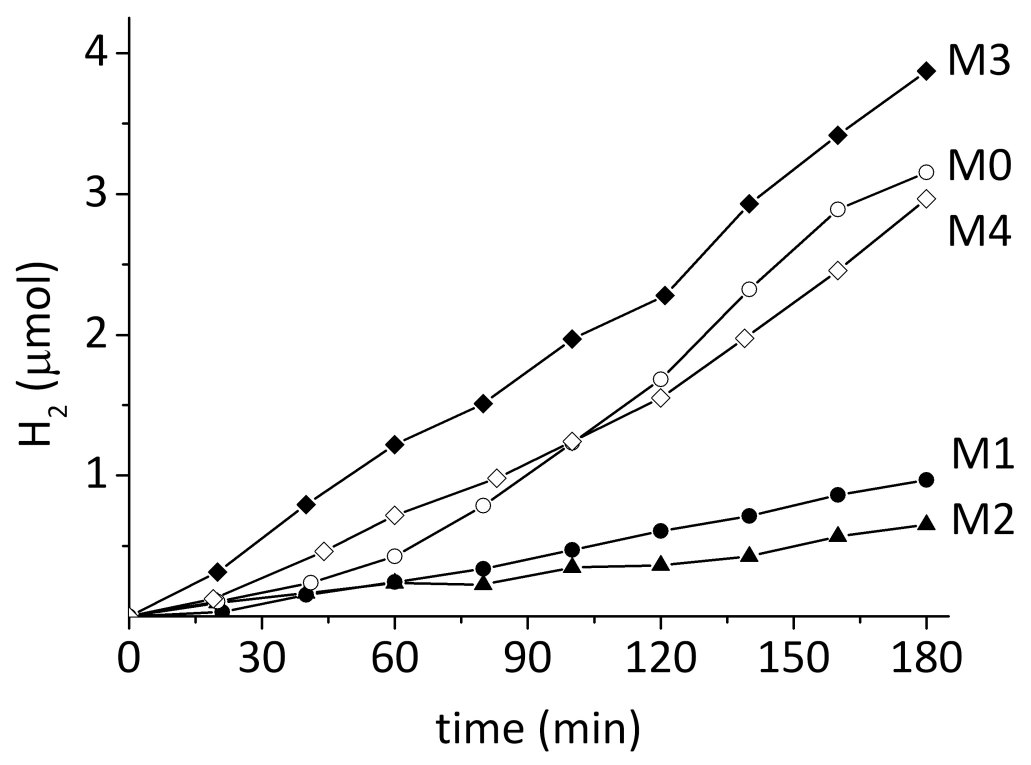


From the point of view of application for solar fuel production, the most important data is that provided in Figure 4.7. According to these data, upon solar light irradiation the photocatalytic activity decreases considerably with respect to anatase (M0) after $\mathrm{NaOH}$ digestion and formation of the titanate nanotubes (M1). The sample annealed at $500{ }^{\circ} \mathrm{C}(\mathrm{M} 3)$ results in the most efficient photocatalyst under simulated solar light. It should be emphasised that the photocatalytic activity of these titanium-containing samples has been measured in the absence of Pt as co-catalysts. Under these conditions, M3 is notably more active than the "black titania" sample M4.

In order to understand the origin of the better performance of M3 for hydrogen production under solar light irradiation, the information of hydrogen production upon 532 or $355 \mathrm{~nm}$ laser irradiation can be used. Solar light contains about $4 \%$ of energy in the UV and about $42 \%$ in the visible region. However, it could be that just the small percentage of UV light could be responsible for most of the photocatalytic activity, while, on the other hand, this wavelength range could only have a marginal contribution to the overall photoresponse in spite of visible light absorption of the samples.

To prepare efficient titanium photocatalyst, the target is to obtain materials with visible light photoresponse. Thus, the results of hydrogen production at $355 \mathrm{~nm}$ corresponds to UV irradiation where of titanium samples absorb, while $532 \mathrm{~nm}$ irradiation will report on the visible light photoresponse of the samples under study. In other words, photocatalytic data of hydrogen generation with $355 \mathrm{~nm}$ laser would report on the "intrinsic" photocatalytic activity upon irradiation with photons of energy higher than the band gap of the semiconductor. On the other hand, the photocatalytic data under $532 \mathrm{~nm}$ irradiation (green light) indicates the visible light photoresponse of the samples.

Comparison of the photoactivity of Figures 4.5. and 4.6. shows that the ranking of photocatalytic activity is totally different. Irradiation at $355 \mathrm{~nm}$ results in M0 as the most active sample with similar activity as M4, but higher than that of M3 or the titanate samples M1 and M2. This indicates that for UV wavelength the colour of the samples does not have any influence on the photocatalytic activity and white M0 is the most efficient. What is important in this UV range seems to be the crystallinity of the samples and the absence of defects in its structure. $\mathrm{NaOH}$ 
treatment or $\mathrm{H}_{2}$ annealing at low temperatures will produce defect in the structure and will decrease the UV photocatalytic activity. In contrast, the ranking with visible light can be rationalized as a function of absorbance in the visible. Accordingly, the most efficient sample is M3, while the photocatalytic activity of M0 is negligible (see Figure 4.5.).

Thus, it seems that the photocatalytic data under solar light irradiation is the result of the weighed contribution between crystallinity and visible light absorption. Accordingly M3 is the most efficient sample, because it combines both properties. M0 is the second most efficient samples due to its high UV photoresponse derived from its crystallinity. The photocatalytic activity data, particularly the poor performance of sample M1 and M2 indicated that the surface area does not play a prevalent role in the photocatalytic activity since these two materials have almost one order of magnitude higher area that M0 or M4. Also visible light absorption does not guarantee high solar light photocatalytic activity, if the sample is not highly crystalline.

In order to provide further support to the relative contribution of UV and visible light photoresponse in sample M3, the photoresponse spectrum for hydrogen generation was measured. Figure 4.8. provides a comparison of the photocatalytic activity of M3 for hydrogen generation and the absorption spectrum. It can be seen there that the two properties are parallel, indicating that for this M3 sample, the absorptivity determines the efficiency in the generation of hydrogen that is higher under UV, but extends clearly into the visible region. 
Figure 4.8. Photocatalytic hydrogen production from water-methanol (3:1) mixtures as a function of the irradiation wavelength (vertical bars) for sample M3. The continuous line corresponds to the absorption spectrum of this sample.

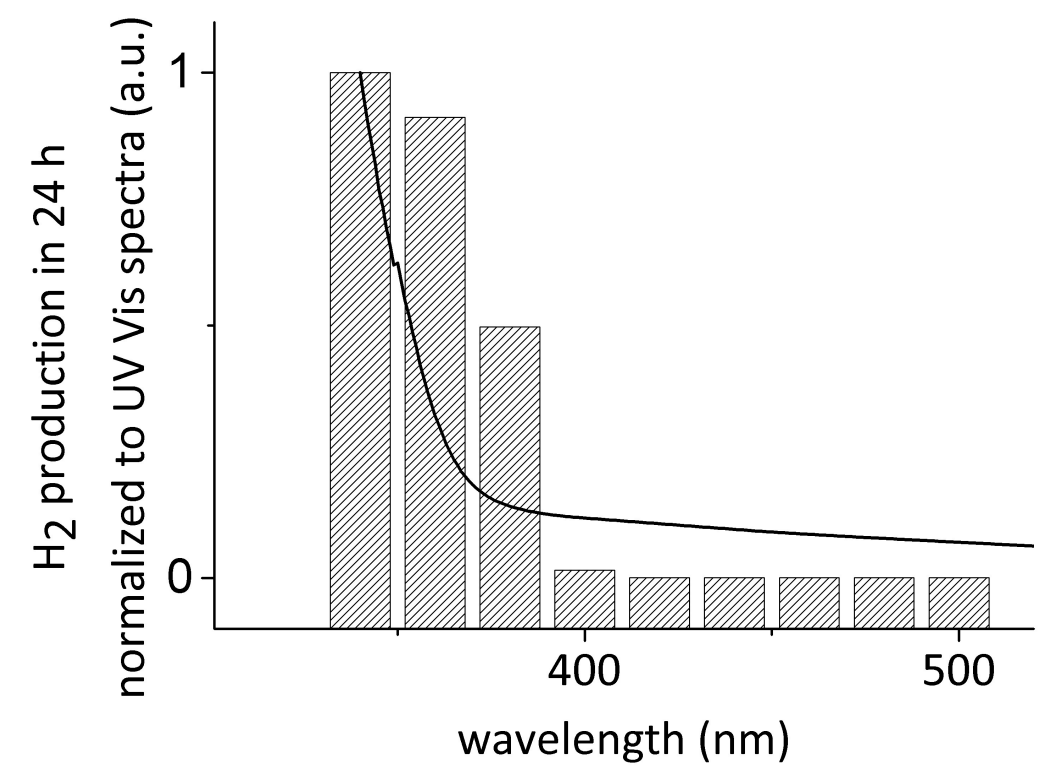

\subsection{Conclusions.}

In the present manuscript we have shown that crystal and morphological changes leading from particle to nanotubes due to $\mathrm{NaOH}$ digestion are accompanied by colour changes in titania and that hydrogen annealing increases the intensity of colour. High temperature treatment promotes the recovery of anatase crystal phase. In this way, samples with black appearance (M3-M4), but having different preparation procedure exhibit different photocatalytic activity. Also our study has emphasized that the ranking of photocatalytic activity depends remarkably on the excitation wavelength, particularly if the irradiation light corresponds to the visible region or contains some UV wavelengths and that solar light photocatalytic activity can be explained as the balanced contribution of the response in the UV and visible regions. 


\subsection{References.}

[1] Bard, A. J. Fox, M. A. Accounts of Chemical Research 1995, 28, 141-145.

[2] Bensaid, S. Centi, G. Garrone, E. Perathoner, S. Saracco, G. ChemSusChem 2012, 5, 500-521.

[3] Chen, X. Shen, S. Guo, L. Mao, S. S. Chemical Reviews 2010, 110, 6503-6570.

[4] Dunn, S. International Journal of Hydrogen Energy 2002, 27, 235-264.

[5] Esswein, A. J. Nocera, D. G. Chemical Reviews 2007, 107, 4022-4047.

[6] Navarro, R. M. Sanchez-Sanchez, M. C. Alvarez-Galvan, M. C. del Valle, F. Fierro, J. L. G. Energy \& Environmental Science 2009, 2, 35-54.

[7] Ni, M. Leung, M. K. H. Leung, D. Y. C. Sumathy, K. Renewable and Sustainable Energy Reviews 2007, 11, 401-425.

[8] Nowotny, J. Sorrell, C. C. Sheppard, L. R. Bak, T. International Journal of Hydrogen Energy 2005, 30, 521-544.

[9] Bakardjieva, S. Å ubrt, J. Å tengl, V. Dianez, M. J. Sayagues, M. J. Applied Catalysis B: Environmental 2005, 58, 193-202.

[10] Di Paola, A. MarcÃ, G. Palmisano, L. Schiavello, M. Uosaki, K. Ikeda, S. Ohtani, B. Journal of Physical Chemistry B 2002, 106, 637-645.

[11] Hurum, D. C. Agrios, A. G. Gray, K. A. Rajh, T. Thurnauer, M. C. Journal of Physical Chemistry B 2003, 107, 4545-4549.

[12] Ohno, T. Akiyoshi, M. Umebayashi, T. Asai, K. Mitsui, T. Matsumura, M. Applied Catalysis A: General 2004, 265, 115-121.

[13] DvoranovÃ $\tilde{i}_{i}$, D. BrezovÃ $\tilde{i}_{i}$, V. MazÃo${ }^{\circ}$, M. Malati, M. A. Applied Catalysis B: Environmental 2002, 37, 91-105.

[14] Irie, H. Watanabe, Y. Hashimoto, K. Chemistry Letters 2003, 32, 772-773.

[15] Xu, A. W. Gao, Y. Liu, H. Q. Journal of Catalysis 2002, 207, 151-157.

[16] Janisch, R. Gopal, P. Spaldin, N. A. Journal of Physics Condensed Matter 2005, 17, R657-R689.

[17] Asahi, R. Morikawa, T. Ohwaki, T. Aoki, K. Taga, Y. Science 2001, 293, 269271.

[18] Zhang, M. Jin, Z. Zhang, J. Guo, X. Yang, J. Li, W. Wang, X. Zhang, Z. Journal of Molecular Catalysis A: Chemical 2004, 217, 203-210.

[19] Aprile, C. Corma, A. Garcia, H. Physical Chemistry Chemical Physics 2008, 10, 769-783. 
[20] Chen, X. Liu, L. Yu, P. Y. Mao, S. S. Science [Washington, DC, U. S.] 2011, 331, 746-750.

[21] Danon, A. Bhattacharyya, K. Vijayan, B. K. Lu, J. Sauter, D. J. Gray, K. A. Stair, P. C. Weitz, E. ACS Catal. 2012, 2, 45-49.

[22] Toledo-Antonio, J. A. Cortes-Jacome, M. A. Orozco-Cerros, S. L. MontielPalacios, E. Suarez-Parra, R. Angeles-Chavez, C. Navarete, J. Lopez-Salinas, E. Appl. Catal., B 2010, 101, 747.

[23] Tsai, C.-C. Teng, H. Chem. Mater. 2006, 18, 367-373.

[24] Xiong, L.-B. Li, J.-L. Yang, B. Yu, Y. J. Nanomater. 2011, 831524, 13 pp.

[25] Nagaveni, K. Sivalingam, G. Hegde, M. S. Madras, G. Environmental Science and Technology 2004, 38, 1600-1604.

[26] Zhang, Q. Gao, L. Guo, J. Applied Catalysis B: Environmental 2000, 26, 207215. 
Chapter 5

Synergy of the combination of titanate nanotubes with titania nanoparticles for the photocatalytic hydrogen generation from water methanol mixture using simulated sunlight. 
Chapter 5 - Synergy of the combination of titanate nanotubes with titania nanoparticles for the photocatalytic hydrogen generation from water methanol mixture using simulated sunlight.

\subsection{Introduction.}

As commented in the introduction, photocatalysis applied to solar fuel production is a topic of much current interest and a long-term alternative to fossil fuels. ${ }^{[1-5]}$ Considering the current low efficiency of the photocatalytic activity in solar fuel production enhancement of the efficiency is a continuous task. A strategy to improve the efficiency of a photocatalytic system is to combine two semiconductor in intimate contact in such a way that photoexcitation of one of the components can lead to heterojunction electron transfer between the two component. ${ }^{[6-9]}$ The case that has been more extensively studied corresponds to the use of two different semiconductors such as quantum dots supported on titanium dioxide or transition metal oxide including $\mathrm{RuO}_{2}$ and $\mathrm{WO}_{3}$, on titania.[10] While the titanium dioxide in the anatase phase or the commercial Evonik P25 having a proportion of about $20 \% / 80 \%$ rutile/anatase is the most widely used photocatalyst, the possibility to enhance the photocatalytic activity of this material by adding a second different form of titanium dioxide remains to be explored. In the present chapter we will show the synergy in the photocatalytic activity derived from combining $\mathrm{TiO}_{2}$ nanoparticles with titanate nanotubes. The present case is also another example of optimization of the photocatalytic activity of $\mathrm{TiO}_{2}$, in this case by adding a different form of titanium oxide.

This synergy between titanate nanotubes and titania nanoparticles has been observed for two different types of commercial titanium dioxide nanoparticles, both in the absence and in the presence of platinum or gold as co-catalyst. The interest of this research is to show the possibility that the combination of various forms of titanium-containing materials offer to optimize the photocatalytic efficiency. This synergy seems to arise from the interfacial charge transfer between the two titanium semiconductors. We have demonstrated the applicability of this strategy based on exclusive use of titanium-containing materials for the hydrogen generation from water-methanol mixtures using simulated solar light that is a 
process of large interest in the context of conversion sunlight into chemical energy.[11-17]

\subsection{Result and discussion.}

It had been reported that digestion of $\mathrm{TiO}_{2}$ nanoparticles in strong alkali conditions leads to the formation of titanates appearing with morphology of nanotubes with long aspect-ratio.[18] The average length dimension is several hundred nanometres, with diameters lower than $10 \mathrm{~nm}$. We have been able to reproduce this remarkable morphological change from nanoparticles to nanotubes. Figure 5.1. shows representative TEM images of the resulting titanate nanotubes.

This change in morphology is accompanied by a change in the X-ray diffraction pattern, indicating that $\mathrm{NaOH}$ digestion has produced a phase transition from anatase to titanate structure as presented in Figure 5.2.

Figure 5.1. Diameter distribution and TEM image of the titanate nanotubes obtained in the present chapter.

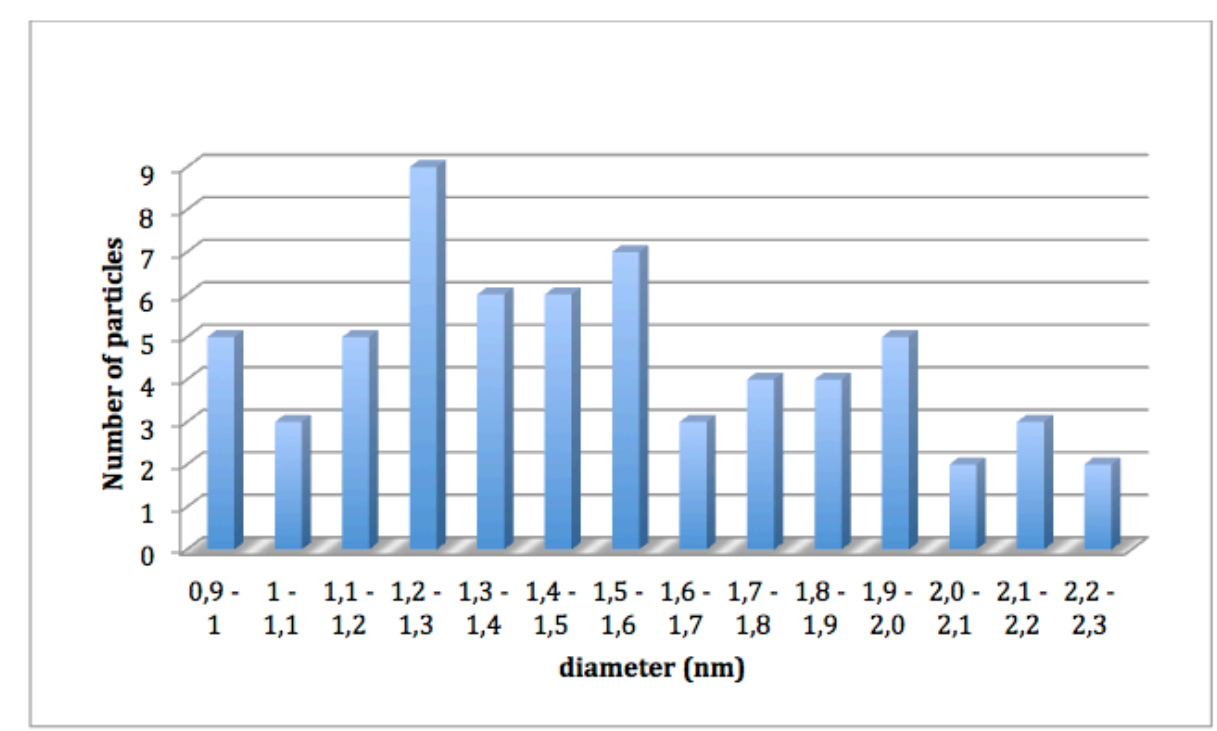




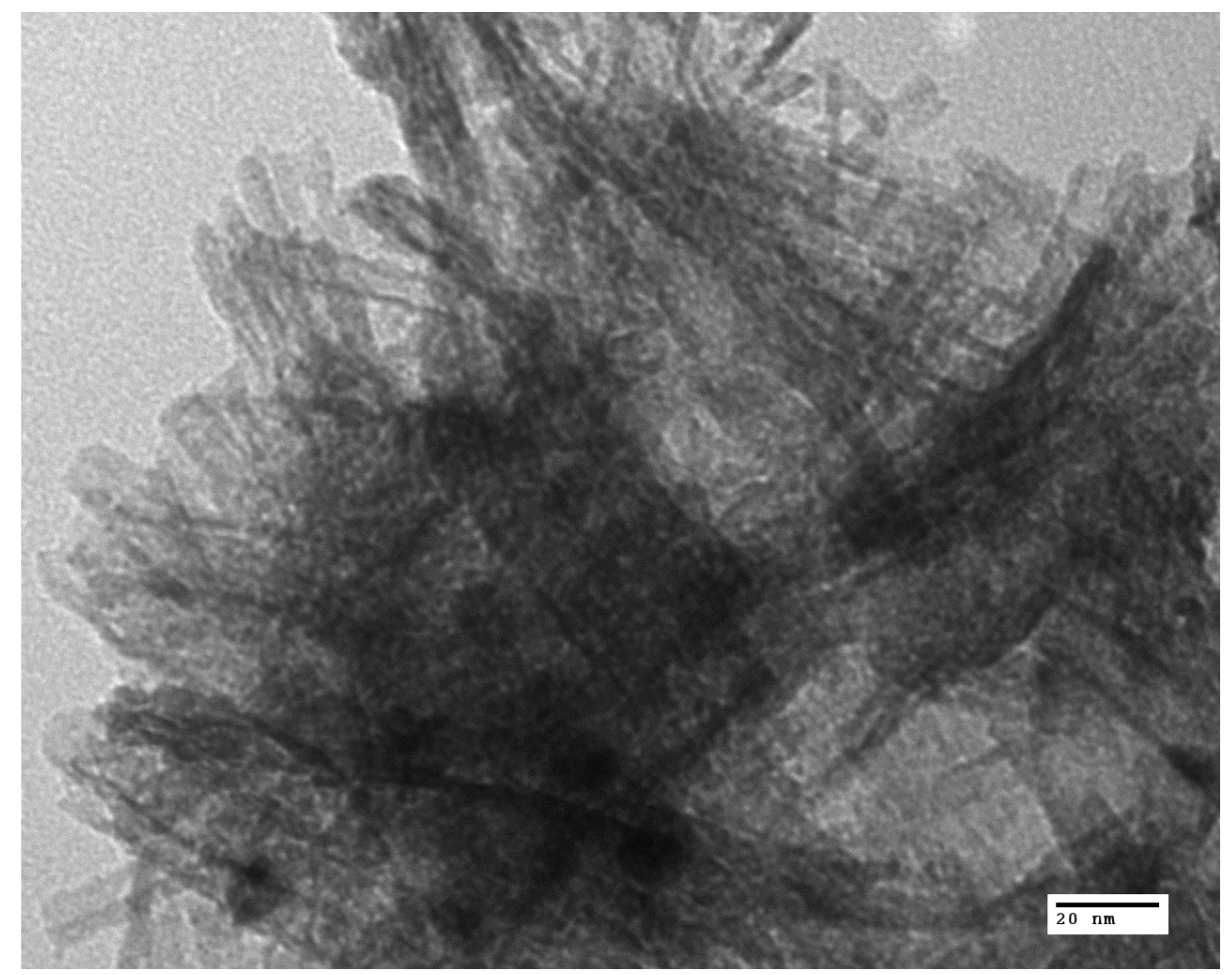

Figure 5.2. XRD of the titanium-containing samples. See table 5.1. for the sample code.

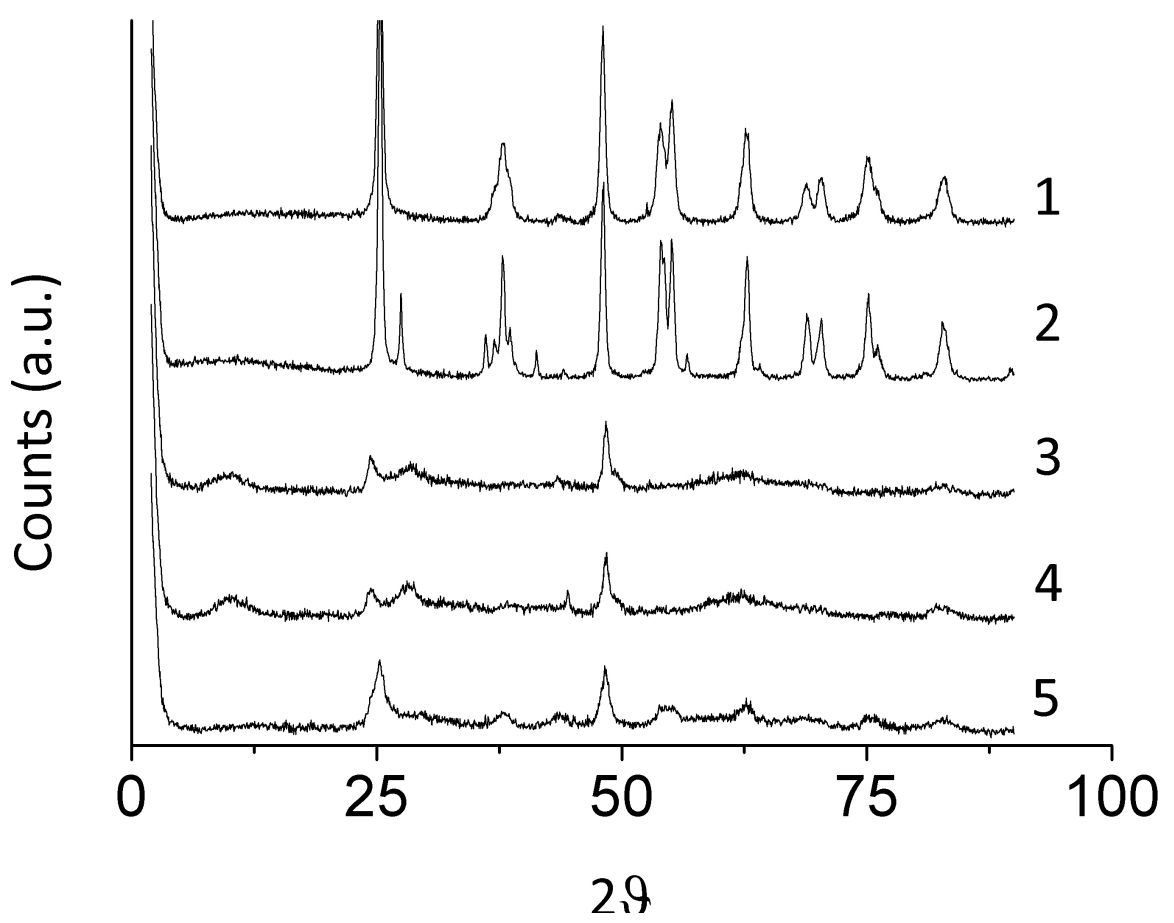


As shown in Figure 5.3., a remarkable feature of these titanate nanotubes, besides the long aspect ratio, is the appearance of a residual optical absorption spanning into the visible region with a decreasing absorptivity towards long wavelengths.

Figure 5.3. Diffuse reflectance UV-Vis absorption spectra (plotted as the KubelkaMunk function of the remittance (R)) of the set of titanium samples under study. Refer to table 5.1. for the sample code.

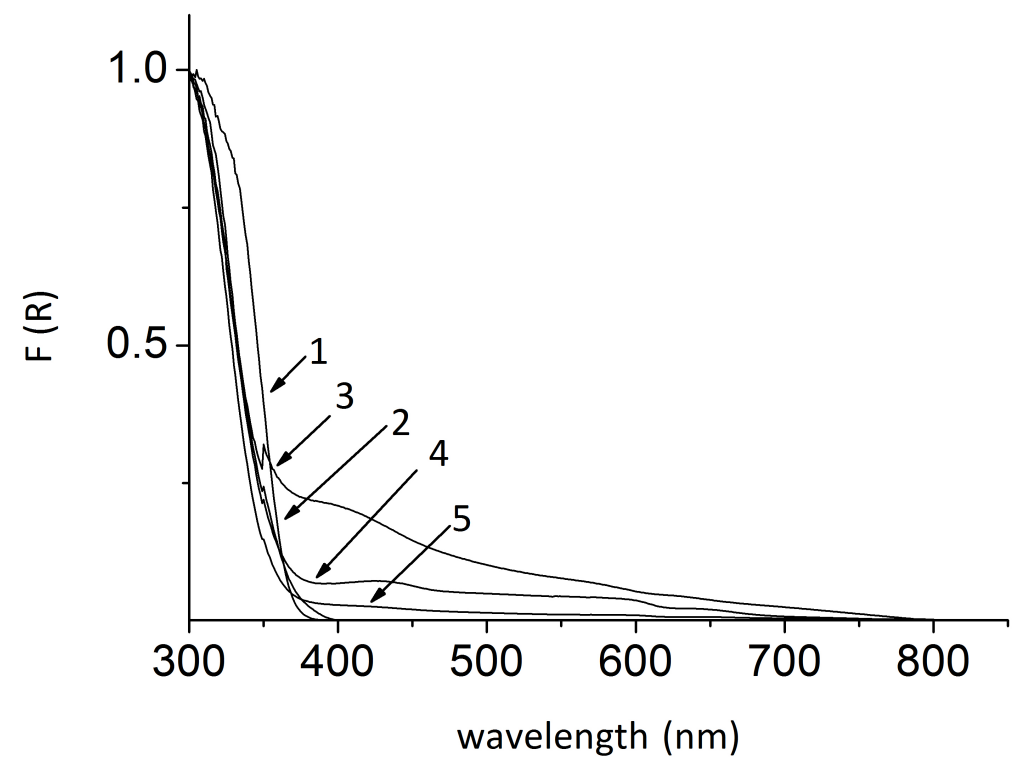

This optical absorption had not been mentioned in the original report of the preparation of these materials.[18]. By analogy with the reported black color for some titanium dioxide, ${ }^{[19]}$ we propose similarly that the brown colour of titanate nanotubes is due to the formation of a thin amorphous layer on the titanate nanotubes onto crystalline layers. This proposal is parallel to that recently suggested to rationalize that black colour observed for well crystalline titanium dioxide samples obtained by submitting small titanium dioxide nanoparticles to thermal treatment at $500{ }^{\circ} \mathrm{C}$ temperature under and hydrogen atmosphere. ${ }^{[19]}$ In the chapter 4 , we have shown that these titanate nanotubes with optical absorption in the visible region can exhibit activity for hydrogen generation from water methanol mixtures with visible light. In the present case we reasoned that the combination of these titanate nanotubes in a certain percentage with titanium 
dioxide nanoparticles could lead to an improved photocatalytic system due to the formation of bulk heterojunction, even for different titanium-containing particles. To test this hypothesis we selected two different commercial titanium dioxide nanoparticles that had been widely used as photocatalysts, both for dye degradation and hydrogen generation, namely Evonik P25 and Millenium PC500 $\mathrm{TiO}_{2}$. To each of these two nanoparticulate titania dioxide samples, increasing proportion of titanate nanotubes from 0 to $100 \%$ were added, and the resulting mixtures were evaluated for hydrogen production under ambient conditions using simulated solar light. Figure 5.4. shows the temporal profiles of hydrogen evolution for some selected mixtures, while Table 5.1. compiles all the data of hydrogen productivity as a function of the composition of the photocatalyst.

Figure 5.4. Photocatalytic hydrogen production from water-methanol (3:1) mixtures of selected samples as photocatalysts under simulated sunlight irradiation. Reaction conditions: Water-methanol (3:1) mixtures $25 \mathrm{~mL}$, catalyst concentration $1.0 \mathrm{~g} \times \mathrm{L}^{-1}$. Refer to Table 5.1. for sample codes and a complete set of hydrogen production values.

A) Samples containing titanate nanotubes and Millenium PC500 $\mathrm{TiO}_{2}$ particles. B) Samples containing titanate nanotubes and Evonik $\mathrm{P} 25 \mathrm{TiO}_{2}$ particles.

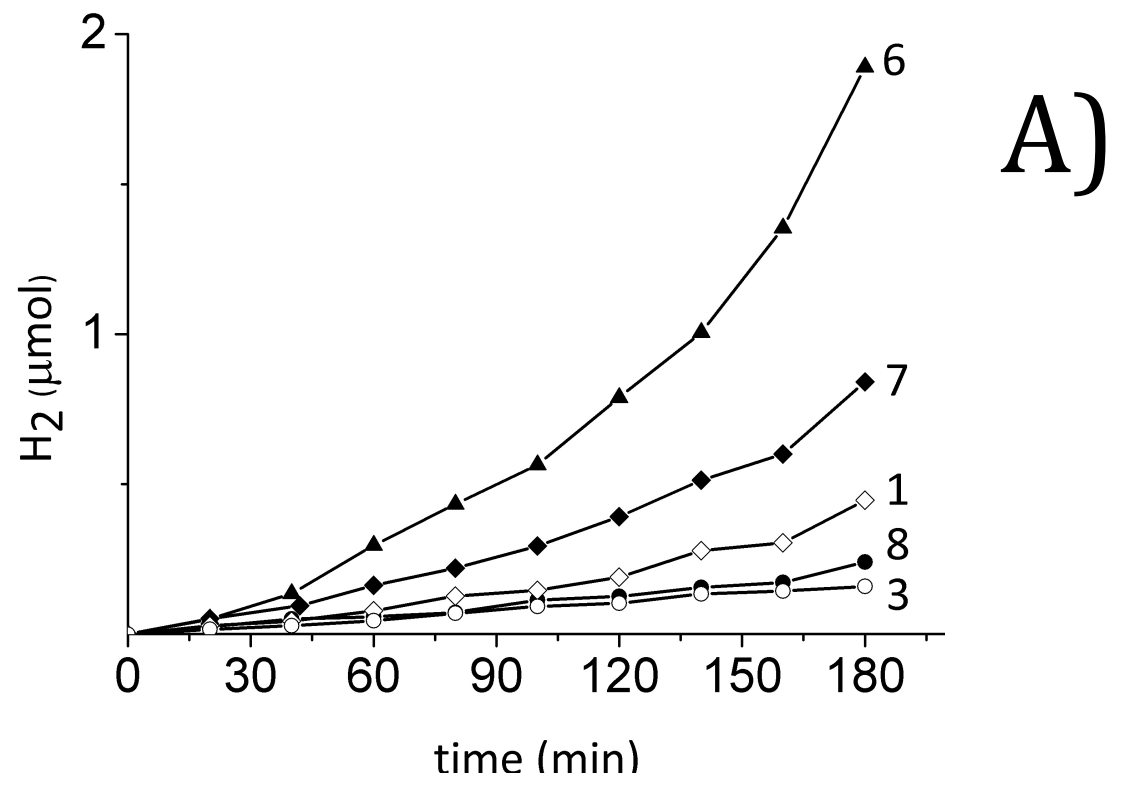




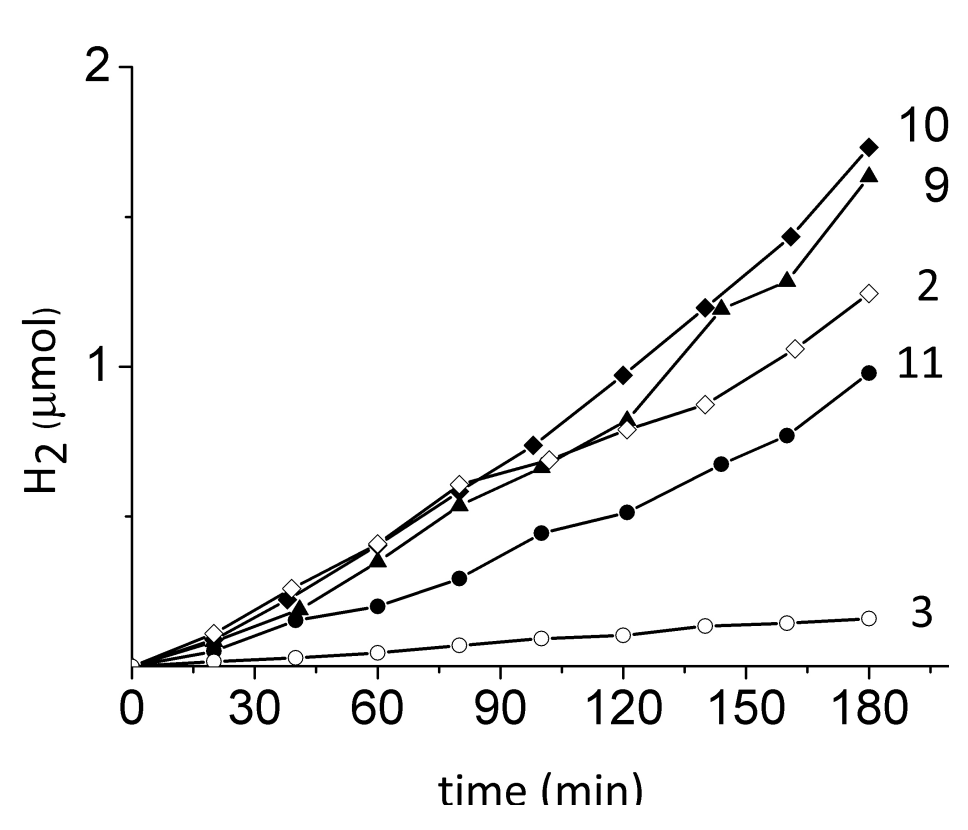

B)

The results indicate that pure Evonik P25 or Millennium PC500 $\mathrm{TiO}_{2}$ exhibit lower productivity for hydrogen generation than mixtures in where titanate nanotubes are present. The optimal efficiency corresponds to $12 \%$ or $6 \%$ titanate for Evonik P25 or Millennium $\mathrm{TiO}_{2}$, respectively. Interestingly larger proportion of titanate nanotubes is detrimental for the photocatalytic efficiency.

It should be noted that the results provided in Figure 5.4. and Table 5.1. (entry 111) corresponds to photocatalytic mixture in which platinum or another noble metal was not present. It well known in the state of the art that the presence of platinum or gold enhances considerably the photocatalytic efficiency of titania by acting as cocatalyst for the evolution of hydrogen gas and by providing a Schottky barrier reducing electron-hole recombination. ${ }^{[20,21]}$ In view of this, it is also of interest to check whether or not the synergy that has been observed between titanate nanotubes and titanium dioxide also takes place for samples containing some noble metal as cocatalyst. Furthermore, since the amount of noble metal present in the semiconductor should be minimized as much as possible, it is of interest to determine the relative efficiency in terms of the amount of noble metal present in the system.

In order to determine the influence of noble metals on the photocatalytic activity, we prepared titanate nanotubes containing platinum or gold nanoparticles that 
have been supported by deposition-precipitation method[22, 23] starting from aqueous solution of chloroplatinic or chloroauric acids at $\mathrm{pH} 10$. The platinum or gold content of titanate nanotubes determined by chemical analysis is about $0.3 \mathrm{wt} . \%$ and $0.5 \mathrm{wt} \%$, respectively. These samples containing platinum or gold nanoparticles supported on modified nanotubes were used to prepare mixtures with Evonik P25 and Millenium PC500 titania in proportions ranging from 6 to 100 wt.\%. The results of photocatalytic hydrogen generation of these mixtures containing some platinum or gold are also compiled in Table 5.1., while Figure 5.5. shows the temporal evolution of hydrogen under simulated solar light irradiation.

Figure 5.5. Photocatalytic hydrogen production from water-methanol (3:1) mixtures of selected samples as photocatalysts under simulated sunlight irradiation. Reaction condition: Water-methanol (3:1) mixtures $25 \mathrm{~mL}$, catalyst concentration $1,0 \mathrm{~g} \times \mathrm{L}^{-1}$. Refer to Table 5.1. for the sample codes and a complete set of hydrogen production values

A) Samples containing gold nanoparticles supported on titanate nanotubes and Millenium PC500 $\mathrm{TiO}_{2}$ particles. B) Samples containing platinum nanoparticles supported on titanate nanotubes and Millenium PC500 $\mathrm{TiO}_{2}$ particles. C) Samples containing platinum nanoparticles supported on titanate nanotubes and Evonik P25 $\mathrm{TiO}_{2}$ particles.

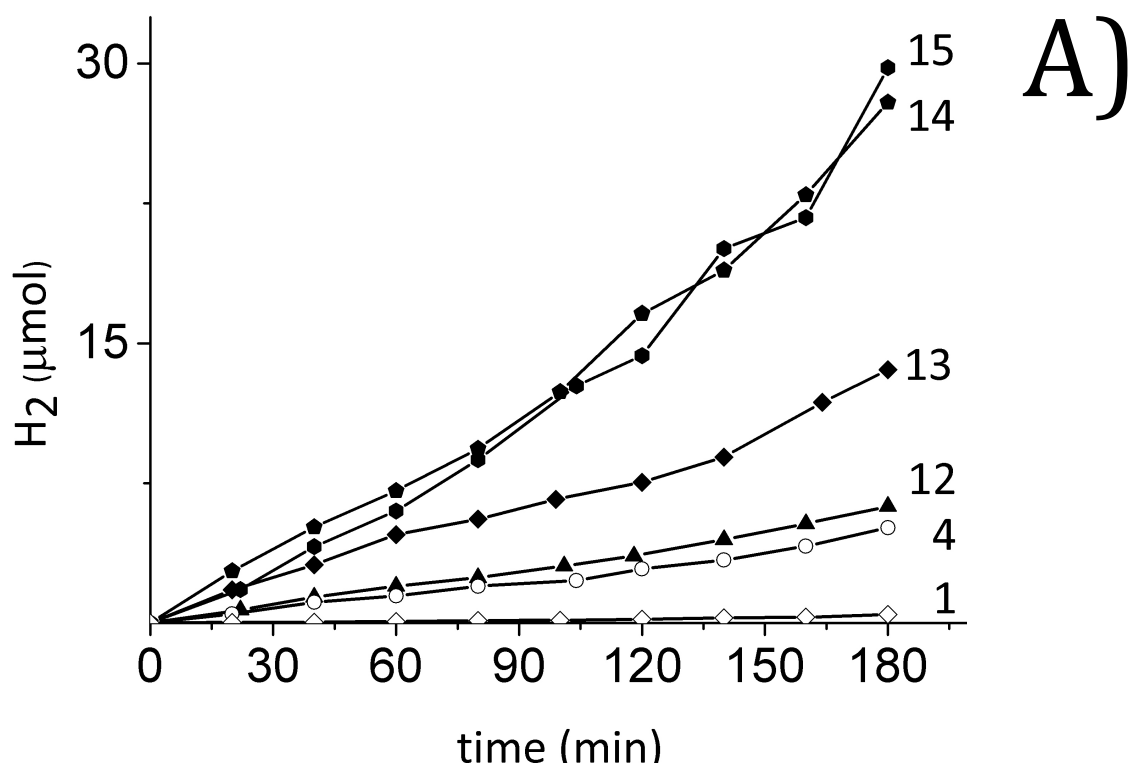



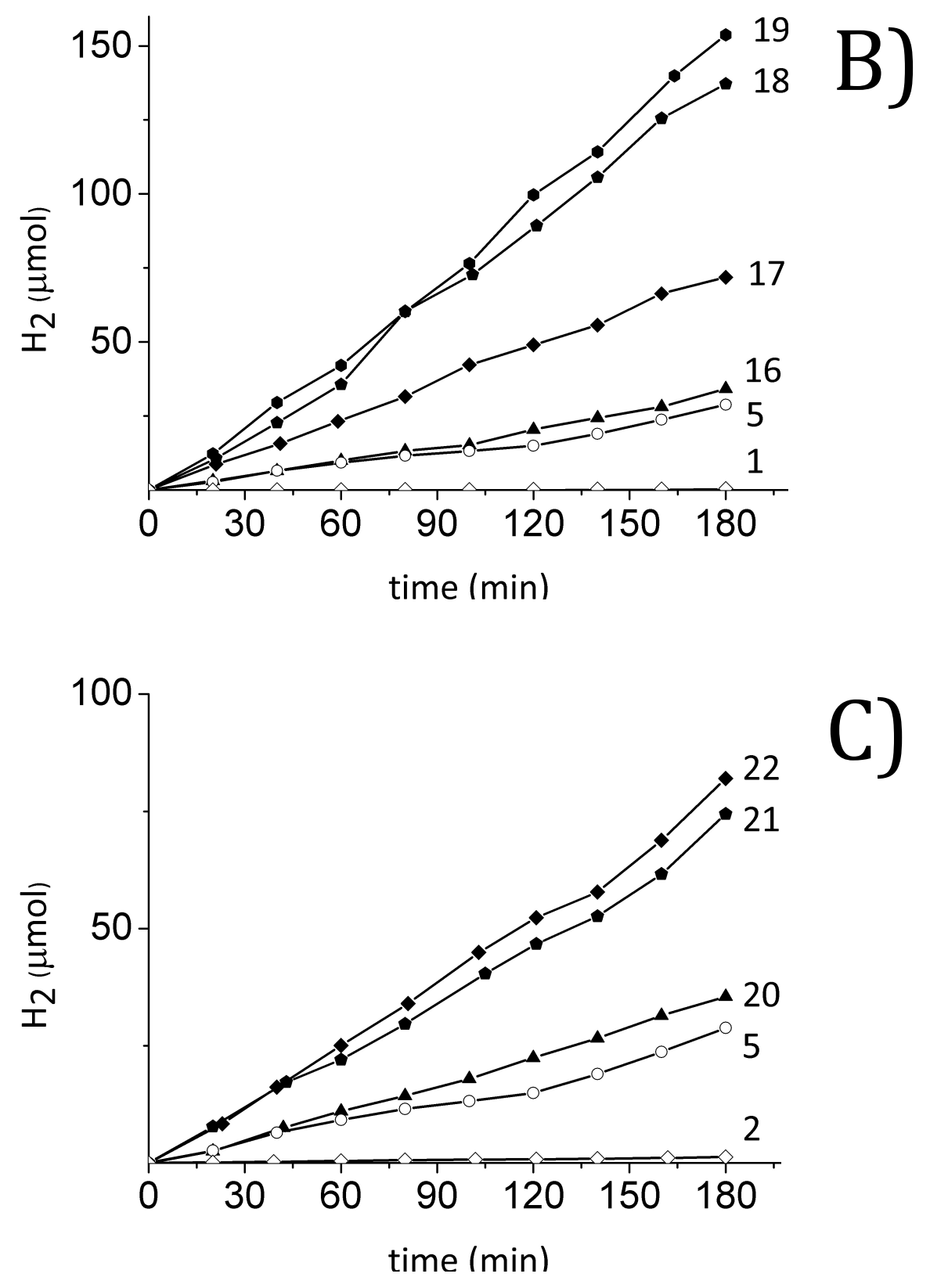
Table 5.1. Code, sample composition and photocatalytic data of the samples under study.

\begin{tabular}{|c|c|c|c|}
\hline Sample & Mix composition (\%wt) & $\begin{array}{l}\text { Metal } \\
\text { Loading } \\
\text { (\%wt) }\end{array}$ & $\begin{array}{l}\mathrm{H}_{2} \quad \text { production } \\
(\mu \mathrm{mol}) \text { (a) }\end{array}$ \\
\hline 1 & $100 \%$ Millenium PC $500 \mathrm{TiO}_{2}$ & - & 0.44 \\
\hline 2 & $100 \%$ Evonik P25 $\mathrm{TiO}_{2}$ & - & 1.2 \\
\hline 3 & $100 \%$ Titanate nanotubes & - & 0.15 \\
\hline 4 & $100 \% \mathrm{Au} \mathrm{NP} /$ Titanate nanotubes (0.5\%wt) & 0.5 & 5.10 \\
\hline 5 & $100 \%$ Pt NP/Titanate nanotubes $(0,3 \% w t)$ & 0.3 & 28.8 \\
\hline 6 & $6 \%$ of $3+94 \%$ of 1 & - & 1.89 \\
\hline 7 & $12 \%$ of $3+88 \%$ of 1 & - & 0.84 \\
\hline 8 & $25 \%$ of $3+75 \%$ of 1 & - & 0.24 \\
\hline 9 & $6 \%$ of $3+94 \%$ of 2 & - & 1.55 \\
\hline 10 & $12 \%$ of $3+88 \%$ of 2 & - & 1.73 \\
\hline 11 & $25 \%$ of $3+75 \%$ of 2 & - & 0.97 \\
\hline 12 & $6 \%$ of $4+94 \%$ of 1 & 0.03 & 6.25 \\
\hline 13 & $12 \%$ of $4+88 \%$ of 1 & 0.06 & 13.57 \\
\hline 14 & $25 \%$ of $4+75 \%$ of 1 & 0.12 & 27.92 \\
\hline 15 & $50 \%$ of $4+50 \%$ of 1 & 0.24 & 29.77 \\
\hline 16 & $6 \%$ of $5+94 \%$ of 1 & 0.018 & 34.19 \\
\hline 17 & $12 \%$ of $5+88 \%$ of 1 & 0.036 & 71.87 \\
\hline 18 & $25 \%$ of $5+75 \%$ of 1 & 0.072 & 137.18 \\
\hline 19 & $50 \%$ of $5+50 \%$ of 1 & 0.144 & 153.71 \\
\hline 20 & $6 \%$ of $5+94 \%$ of 2 & 0.03 & 35.46 \\
\hline 21 & $12 \%$ of $5-88 \%$ of 2 & 0.06 & 74.41 \\
\hline 22 & $25 \%$ of $5-75 \%$ of 2 & 0.12 & 81.99 \\
\hline
\end{tabular}

(a) hydrogen production measured at $3 \mathrm{~h}$ of solar simulator irradiation 
These photocatalytic data of noble metal-containing samples indicate again a synergy due to the combination of the two titanium semiconducting solids. The maximum efficiency with noble metals seems to occur at the highest percentage of platinum containing titanate nanotubes that is observed at $25 \%$, both for Evonik P25 and Millenium PC500 $\mathrm{TiO}_{2}$. This optimized efficiency probably reflects that in addition to the synergy between the two titanium solid, the platinum content is playing an important role as cocatalyst increasing the photocatalytic efficiency. We notice, however, that even in the case of Millenium $\mathrm{PC500}^{\mathrm{TiO}_{2}}$, the platinum content of the optimal photocatalytic mixtures that is present only in the titanate nanotubes is $0.03 \mathrm{wt} . \%$ that is significantly low compared to the typical amounts reported for titanium dioxide. For instance, when the photocatalytic efficiency of the titanate nanotubes-titanium dioxide mixtures containing platinum are compared with the best turnover numbers for platinum deposited either on titanate nanotubes or deposited on titanium dioxide, the benefits of the combination of the two different titanium forms can be demonstrated. Thus, the turnover number for platinum-Millenium $\mathrm{PC} 50 \mathrm{TiO}_{2}$ and platinum- Evonik P25 $\mathrm{TiO}_{2}$ are 961 and 1892 that are lower than the values of 1831 and 1993 corresponding to platinum for the titanate nanotube - Millenium PC500 nanotubes and platinum on titanate -nanotubes- Evonik P25 $\mathrm{TiO}_{2}$, respectively.

The synergy between titanate nanotubes and titania nanoparticles can be explained based on related precedents reporting enhanced photocatalytic efficiency of heterojunction of different semiconductors. In agreement with this hypothesis we propose that light should be preferentially absorbed by titanate nanotubes, since as shown in Figure 5.3. this component has absorption beyond $380 \mathrm{~nm}$ where anatase does not absorb light. After light absorption in titanate nanotubes, charge separation with the generation of electron and holes would take place. Since the mobility of electron in titania semiconductors is larger than holes, it can be that conduction band electrons migrate from the site in where have been generated to the surface of the titanate nanotubes and, then, to the anatase nanoparticle. Interfacial electron migration from the nanotubes to anatase nanoparticles would be the key process enhancing charge separation, minimizing recombination and extending the lifetime of the charge separated state, as it had been reported for other combinations of semiconductors. ${ }^{[24]}$ This mechanism does 
not exclude other possibilities taking place independently in each titanium phase, but would be unique for the combination of titanate nanotubes with titania nanoparticles, thus resulting in a synergy due to the junction of both phases. Scheme 5.1. illustrates the mechanistic proposal to rationalize the higher efficiency of the combination of titanate nanotubes with anatase nanoparticles.

Scheme 5.1. Proposal for the synergy found for the heterojunction of titanate nanotubes (long line) and titania nanoparticles (spheres) showing the charge separation through heterojunction.

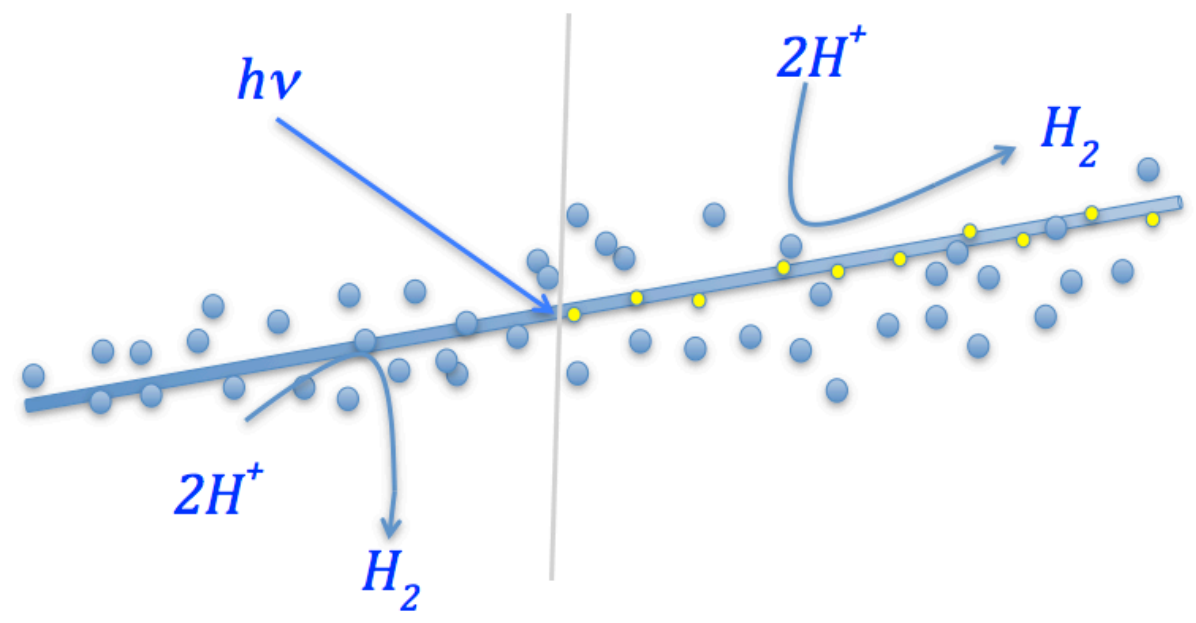

\subsection{Conclusions.}

In the present chapter we provide data showing that the combination of titanate nanotubes, exhibiting an absorption in the visible region, with anatase nanoparticles in the appropriate proportion increases the photocatalytic activity with respect to the activity of the individual components. This synergetic effect is observed both in the absence and in the presence of noble metals platinum and gold nanoparticles acting as co-catalysts for the hydrogen generation and is proposed to derive from the interfacial electron transfer between the titanate nanotubes and titania nanoparticles, our report complements those that have shown that the morphology of titanium base semiconductors plays a role on the photocatalytic efficiency, ${ }^{25}$ since in the present case with have observed that the combination of two different titanium-based semiconductor with different morphology benefits the photocatalytic activity of the system. 


\subsection{References.}

[1] Centi, G.; Perathoner, S., ChemSusChem 2010, 3, 195-208.

[2] Gust, D.; Moore, T. A.; Moore, A. L., Accounts of Chemical Research 2009, 42, 1890-1898.

[3] HammarstroÌ̀m, L.; Hammes-Schiffer, S., Accounts of Chemical Research 2009, 42, 1859-1860.

[4] Roy, S. C.; Varghese, O. K.; Paulose, M.; Grimes, C. A., Acs Nano 2010, 4, 12591278.

[5] Corma, A.; Garcia, H., J. Catal. 2014, 308, 168-175.

[6] Khan, G.; Choi, S. K.; Kim, S.; Lim, S. K.; Jang, J. S.; Park, H., Appl. Catal., B 2013, 142-143, 647-653.

[7] Marschall, R., Adv. Funct. Mater. 2013, Ahead of Print.

[8] Rawal, S. B.; Bera, S.; Lee, D.; Jang, D.-J.; Lee, W. I., Catal. Sci. Technol. 2013, 3, 1822-1830.

[9] Wu, L.; Xing, J.; Hou, Y.; Xiao, F. Y.; Li, Z.; Yang, H. G., Chem. - Eur. J. 2013, 19, 8393-8396.

[10] Sayama, K.; Yoshida, R.; Kusama, H.; Okabe, K.; Abe, Y.; Arakawa, H., Chem. Phys. Lett. 1997, 277, 387-391.

[11] Crabtree, G. W.; Dresselhaus, M. S.; Buchanan, M. V., Physics Today 2004, 57, $39-44$.

[12] Dunn, S., International Journal of Hydrogen Energy 2002, 27, 235-264.

[13] Esswein, A. J.; Nocera, D. G., Chemical Reviews 2007, 107, 4022-4047.

[14] Jensen, S. H.; Larsen, P. H.; Mogensen, M., International Journal of Hydrogen Energy 2007, 32, 3253-3257.

[15] Navarro, R. M.; Sanchez-Sanchez, M. C.; Alvarez-Galvan, M. C.; del Valle, F.; Fierro, J. L. G., Energy \& Environmental Science 2009, 2, 35-54.

[16] Ni, M.; Leung, M. K. H.; Leung, D. Y. C.; Sumathy, K., Renewable and Sustainable Energy Reviews 2007, 11, 401-425.

[17] Nowotny, J.; Sorrell, C. C.; Sheppard, L. R.; Bak, T., International Journal of Hydrogen Energy 2005, 30, 521-544.

[18] Tsai, C.-C.; Teng, H., Chem. Mater. 2006, 18, 367-373. 
[19] Chen, X.; Liu, L.; Yu, P. Y.; Mao, S. S., Science (Washington, DC, U. S.) 2011, 331, 746-750.

[20] Primo, A.; Marino, T.; Corma, A.; Molinari, R.; Garcia, H., Journal of the American Chemical Society 2011, 133, 6930-6933.

[21] Silva, C. G.; Juarez, R.; Marino, T.; Molinari, R.; Garcia, H., Journal of the American Chemical Society 2011, 133, 595-602.

[22] Bamwenda, G. R.; Tsubota, S.; Kobayashi, T.; Haruta, M., Journal of Photochemistry and Photobiology a-Chemistry 1994, 77, 59-67.

[23] Haruta, M., Catalysis Today 1997, 36,153-166.

[24] Serpone, N.; Emeline, A. V.; Horikoshi, S.; Kuznetsov, V. N.; Ryabchuk, V. K., Photochem. Photobiol. Sci. 2012, 11, 1121-1150.

[25] Aprile, C.; Corma, A.; Garcia, H., Physical Chemistry Chemical Physics 2008, 10, 769-783. 
Chapter 6 Visible light photocatalytic activity for hydrogen production from watermethanol mixtures of open-framework V-doped mixed-valence titanium phosphate. 
Chapter 6 - Visible light photocatalytic activity for hydrogen production from water-methanol mixtures of open-framework V-doped mixed-valence titanium phosphate.

\subsection{Introduction.}

As already commented in the introduction of this Thesis, titanium dioxide is the most widely used photocatalyst for pollutant degradation and also for hydrogen generation from water.[1] While $\mathrm{TiO}_{2}$ has many desirable advantages as photocatalyst including affordability, lack of toxicity and stability, we have discussed that main limitation is the lack of photocatalytic response under visible light irradiation. ${ }^{[2-6]}$ As result of this, the overall efficiency of the photocatalytic activity of $\mathrm{TiO}_{2}$ under sunlight irradiation is unsatisfactory and there is considerable room for improvement.[2, 7-14] Among the different strategies to expand the photocatalytic response of $\mathrm{TiO}_{2}$ toward the visible region including deposition of metal nanoparticles exhibiting surface plasmon band [15] and preparation of black titania, ${ }^{[16]}$ the most common approach has been $\mathrm{TiO}_{2}$ doping by metal or non metal elements. ${ }^{[3,17-20]}$ One possibility that has been recently studied has been self-doping of $\mathrm{TiO}_{2}$ by $\mathrm{Ti}^{3+}$ forming blue coloured solids [21] that had been reported to exhibit enhanced photocatalytic activity under visible light irradiation for hydrogen generation from water-methanol mixtures in the presence of Pt nanoparticles as co-catalyst.[21]

There is a continuous interest in the development of new photocatalytic materials that can exhibit comparable or higher activity than $\mathrm{TiO}_{2}$. In this context, in the present chapter, we would like to report the photocatalytic activity for hydrogen generation of open-framework titanium phosphate. Open-framework refers to a crystalline structure with an atomic mass density below the maximum for bulk titanium phosphate $\left(21 \mathrm{amu} / \mathrm{nm}^{3}\right.$ according to ref. $\left.{ }^{[22]}\right)$. One of the advantages of framework metal phosphates as photocatalysts would be the flexibility in their synthesis, their stability in water, the availability of the transition metal and phosphate sources and their easy preparation in multigram scale. In this context, the synthesis of mixed valence $\mathrm{Ti}^{3+} / \mathrm{Ti}^{4+}$ phosphate $\left[\left(\mathrm{Ti}^{3+} / \mathrm{Ti}^{4+}\right) \mathrm{P}\right]$ octahedraltetrahedral framework structure was already reported.[23] The presence of $\mathrm{Ti}^{3+}$ in the structure is responsible for the characteristic deep purple-blue colour of the 
solid that is a prerequisite for visible light photocatalytic activity. In the present chapter, we present characterization and photocatalytic data for hydrogen generation showing that mixed-valence $\mathrm{Ti}^{3+} / \mathrm{Ti}^{4+}$ phosphates behave as semiconductors. Herein, the flexibility in the preparation of open framework phosphates has been exploited for the synthesis of a series of V-doped titanium phosphates with enhanced photocatalytic activity. The state of charge separation upon irradiation has been detected and characterized by transient absorption spectroscopy and the flat band potential of the electron in the conduction band determined by photocurrent measurements against bias voltage. In spite of the high electrocatalytic activity for oxygen evolution of cobalt phosphate, ${ }^{[24]}$ it is surprising that there is only one precedent in the literature reporting the use of iron phosphate for the photocatalytic dye degradation in water.[25] The present results serve to demonstrate the unforeseen behaviour as semiconductor of titanium phosphate, its activity as photocatalyst and the versatility that this material offers for doping leading to the that enhancement of its photocatalytic activity.

\subsection{Results and discussion.}

Open-framework mixed valence $\left(\mathrm{Ti}^{3+} / \mathrm{Ti}^{4+}\right) \mathrm{P}$ was prepared by hydrothermal synthesis in a Teflon-lined autoclave at $170{ }^{\circ} \mathrm{C}$ using elemental titanium, 1,3diaminopropane and phosphoric acid reagents. The $\left(\mathrm{Ti}^{3+} / \mathrm{Ti}^{4+}\right) \mathrm{P}$ yield based on the amount of $\mathrm{Ti}$ metal was about 100\%, in good agreement with the reported synthesis.[23] Three materials (M1-3) were synthetized varying the time of the hydrothermal synthesis from 1, 2 or 4 days (M1, M2, M3 respectively). Addition during the synthesis of vanadium metal in the required amount together with titanium metal powder results in the formation of V-doped mixed valence phosphates (M4-M7). Table 6.1. summarizes the open-framework phosphate materials under study and their main analytical and physical data. For the sake of comparison we also include in the list a $\mathrm{Ti}^{3+}$ doped $\mathrm{TiO}_{2}$ that was prepared according to the literature [21] through hydrothermal synthesis at $220{ }^{\circ} \mathrm{C}$ for $12 \mathrm{~h}$ starting from $\mathrm{Ti}$ metal and hydrochloric acid in aqueous solution. The main characteristics of this doped $\mathrm{TiO}_{2}$ material (M8) are also included in Table 6.1. The success of the synthesis was confirmed by XRD. All the peaks appearing in the XRD 
can be indexed as corresponding to the open-framework $\left(\mathrm{Ti}^{3+} / \mathrm{Ti}^{4+}\right) \mathrm{P}$ synthetized previously by Sevov et al. [23] although we notice variations of the relative peak intensity. Figure 6.1. shows the crystal structure of mixed-valence $\left(\mathrm{Ti}^{3+} / \mathrm{Ti}^{4+}\right) \mathrm{P}$ and the XRD corresponding to the samples M1-M3 obtained at different times of hydrothermal synthesis, showing the same pattern in all cases, but differences in the relative intensity of the peaks. We also notice that the crystallinity of M3 obtained after four days of crystallization is significant lower than sample M1 and, particularly, M2. This could be due to a decrease in the average particle size of the crystals upon prolonged hydrothermal treatment or to a partial conversion of the crystals into amorphous materials.

Figure 6.1. A) Crystal structure of the mixed-valence $\left(\mathrm{Ti}^{3+} / \mathrm{Ti}^{4+}\right) \mathrm{P}$ viewed along the b-axis. Purple atoms correspond to titanium, green to phosphor, blue to oxygen, grey to carbon and light blue to nitrogen. Hydrogen atoms are omitted for clarity. 1,3-diamine appears occupying disordered positions along b-axis channels. B) XRD patterns of the $\left(\mathrm{Ti}^{3+} / \mathrm{Ti}^{4+}\right) \mathrm{P}$ samples $(\mathrm{M} 1-\mathrm{M} 3)$ and the $\left(\mathrm{Ti}^{3+}\right) \mathrm{TiO}_{2}$ sample (M8). C) XRD pattern of the V-doped $\left(\mathrm{Ti}^{3+} / \mathrm{Ti}^{4+}\right) \mathrm{P}$ samples. See Table 6.1. for codes.

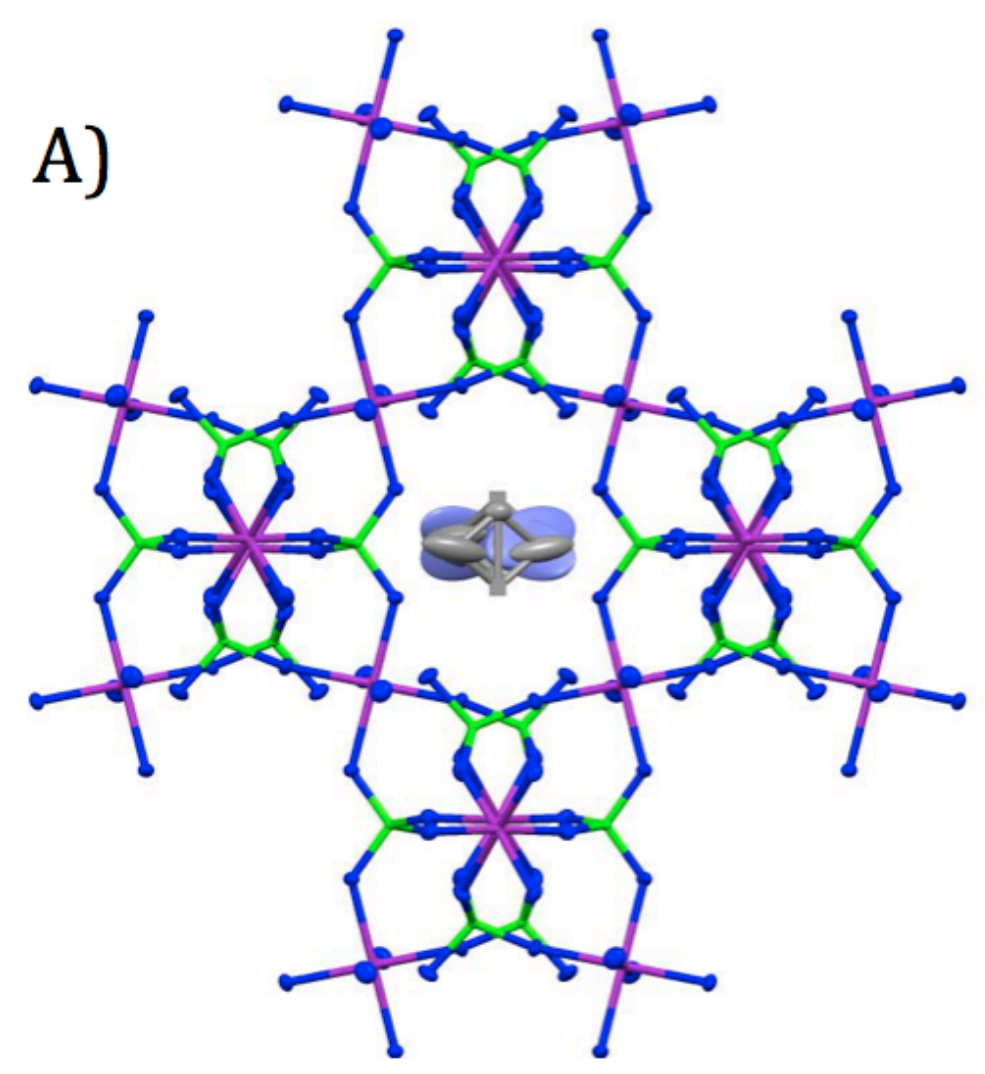



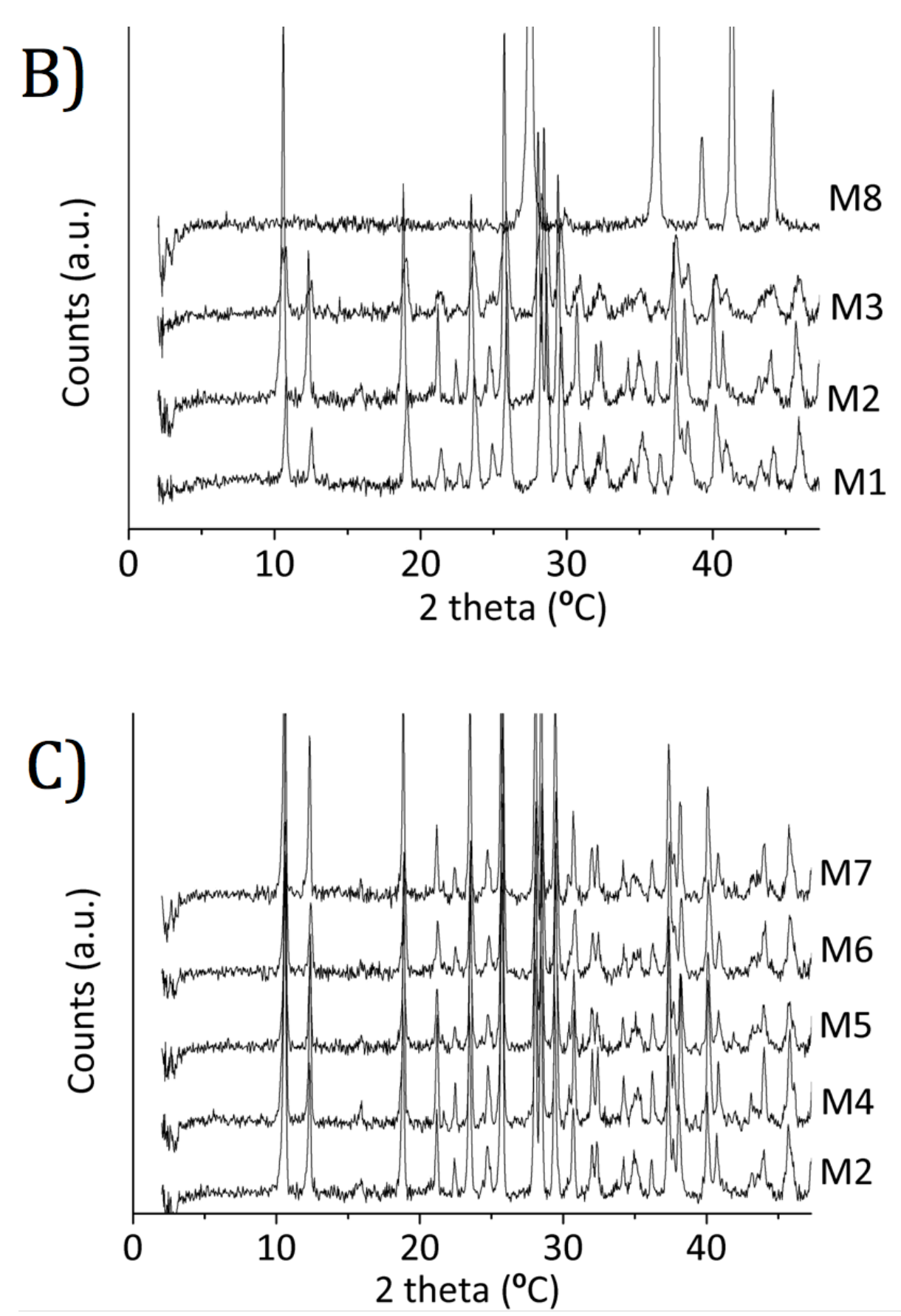

The crystal structure of M1-3 (see Figure 6.1A.) is constituted by $\mathrm{TiO}_{6}$ octahedra and $\mathrm{PO}_{4}$ tetrahedra sharing the edges. The lattice contains Ti-O-P, bonds but not PO-P or Ti-O-Ti bonds. There is an equimolar proportion between $\mathrm{Ti}^{3+}$ and $\mathrm{Ti}^{4+}$, the difference being that the octahedra around $\mathrm{Ti}^{3+}$ have two water molecules in trans position, while for $\mathrm{Ti}^{4+}$ the coordination sphere is occupied by six different phosphate units. The lattice defines channels along the b-axes in which 1,3propandiamines are located occupying disordered positions along the channel. 
In the case of $\mathrm{V}$-doped $\left(\mathrm{Ti}^{3+} / \mathrm{Ti}^{4+}\right) \mathrm{P}, \mathrm{XRD}$ shows again that the samples M4-M7 are highly crystalline, without gradual shift or variation of intensity of the diffraction peaks observed for samples in where the vanadium content increases from 0.25 to $2 \mathrm{wt} \%$. Thus, XRD indicates that the samples should have the same structure commented earlier for $\left(\mathrm{Ti}^{3+} / \mathrm{Ti}^{4+}\right) \mathrm{P}$, except that some positions in the lattice should now be occupied by vanadium ions.

Scanning electron microscopy (SEM) images show that M1-M8 are constituted by large crystals formed by aggregation of flakes having a very smooth surface. Figure 6.2. presents representative images of these materials. In some cases, well-defined prismatic crystallites of about $8 \mu \mathrm{m}$ size can be observed. The samples also contain many much smaller particles without any defined geometry that can derive from degradation of larger crystallites. Electron diffraction of selected areas shows that the material is highly crystalline with well developed diffraction points of high intensity corresponding to a cubic packing in the crystal (see inset Figure 6.2.).

The homogeneous $\mathrm{V}$ distribution in the sample was confirmed by elemental analysis combined with SEM. It was observed that vanadium was present at the corresponding atomic proportion determined by chemical analysis even in areas of $100 \times 100 \mathrm{~nm}^{2}$, both in large crystals as well as in the very small debris.

This homogeneous distribution and particularly the presence of vanadium in large crystals support the homogenous $\mathrm{V}$ distribution as result of the isomorphic substitution in the structure of titanium by vanadium ion. As an example Figure 6.3. shows the results of elemental analysis in selected area of a large crystal indicated in the SEM image as well as in some other selected points of the SEM image (inset Fig. 6.3.). 
Figure 6.2. A) SEM image recorded for M6. Inset (a) electron diffraction image obtained by STEM (b) EDX analysis of the entire area of the sample.
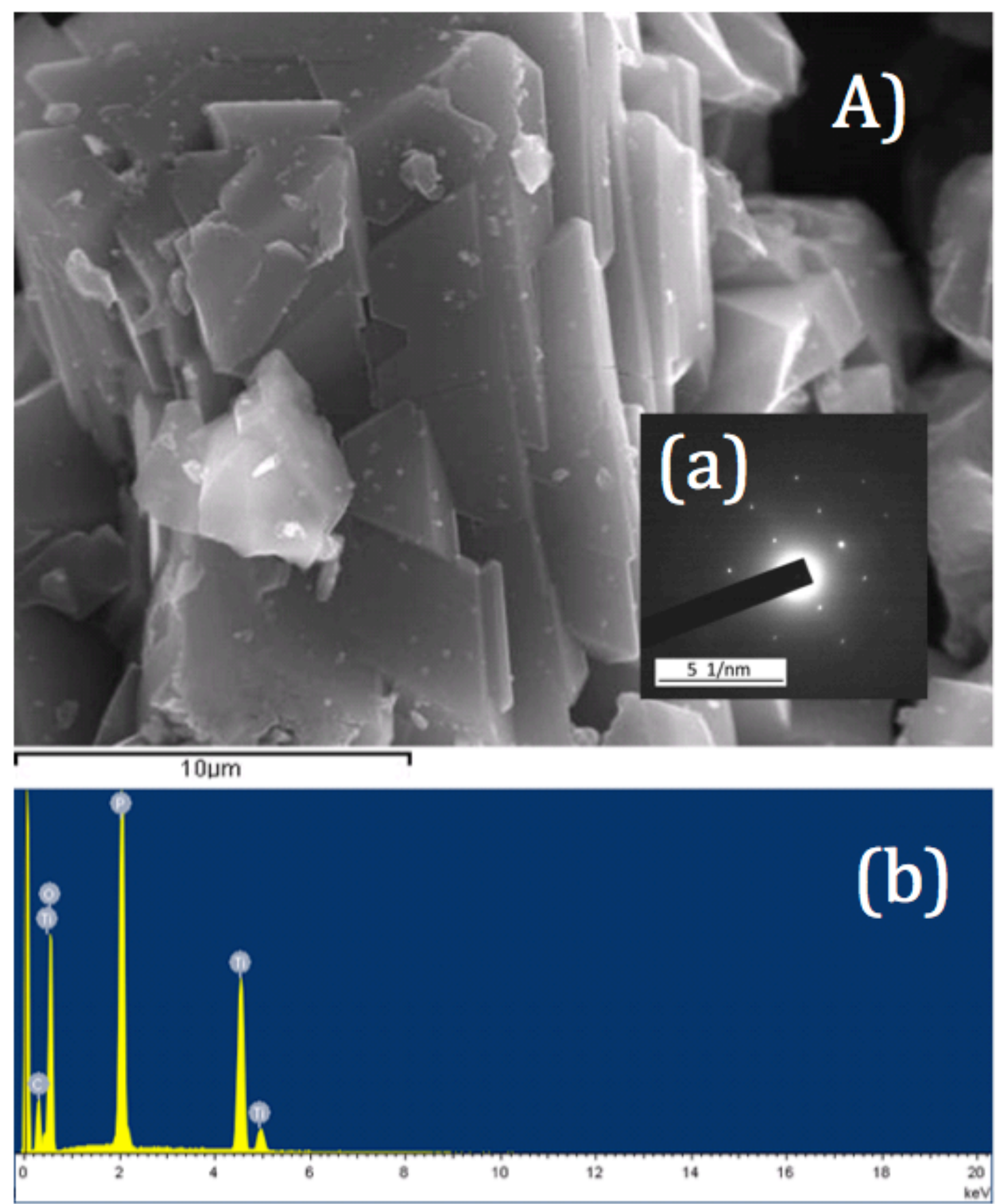
Figure 6.2. B) SEM. image recorded for M2. Inset (a) electron diffraction image obtained with a STEM (b) EDX analysis of the entire area of the sample.

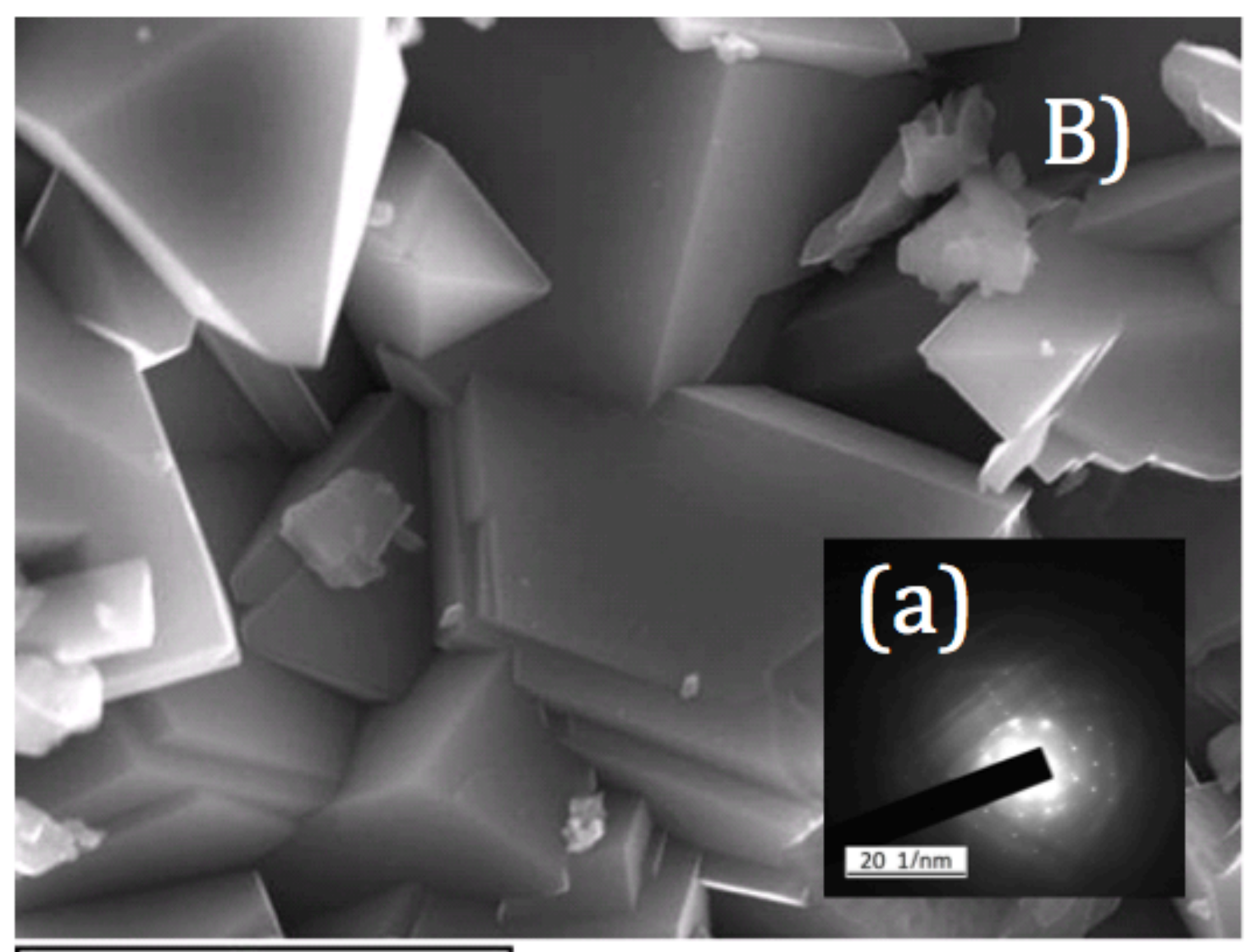

$10 u m$

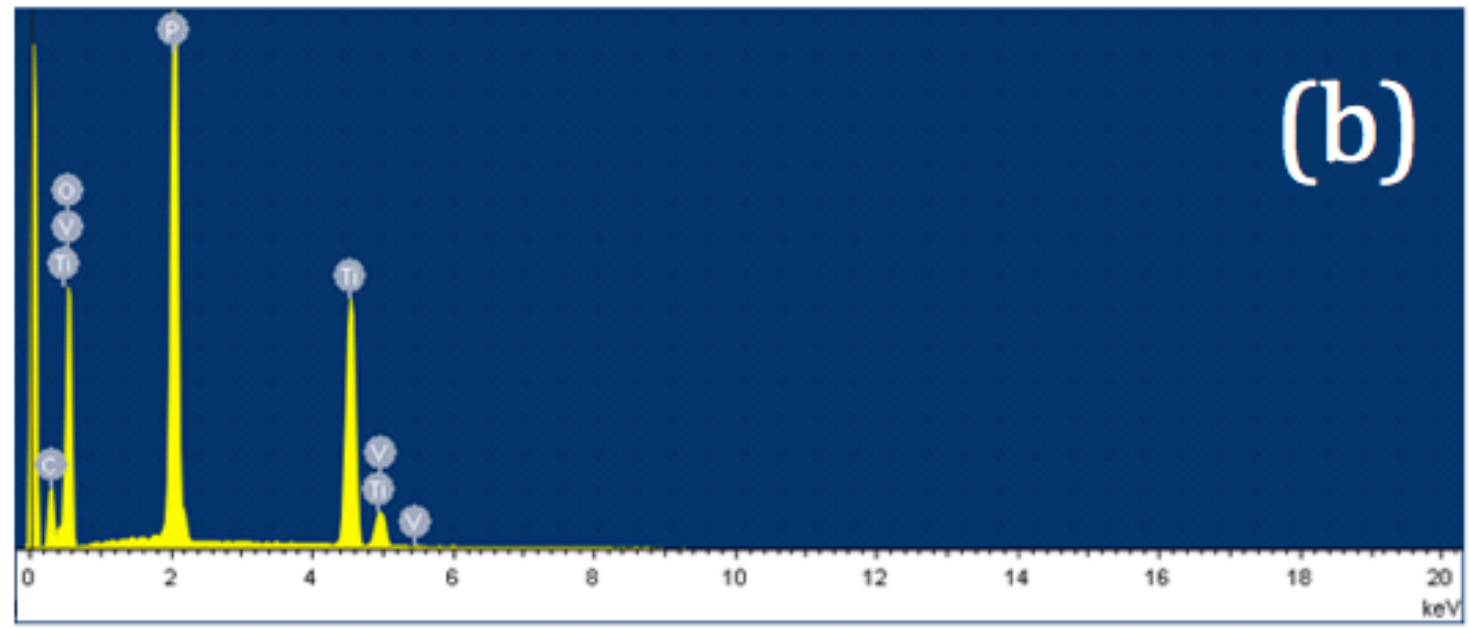


Figure 6.3. SEM image recorded for M6.

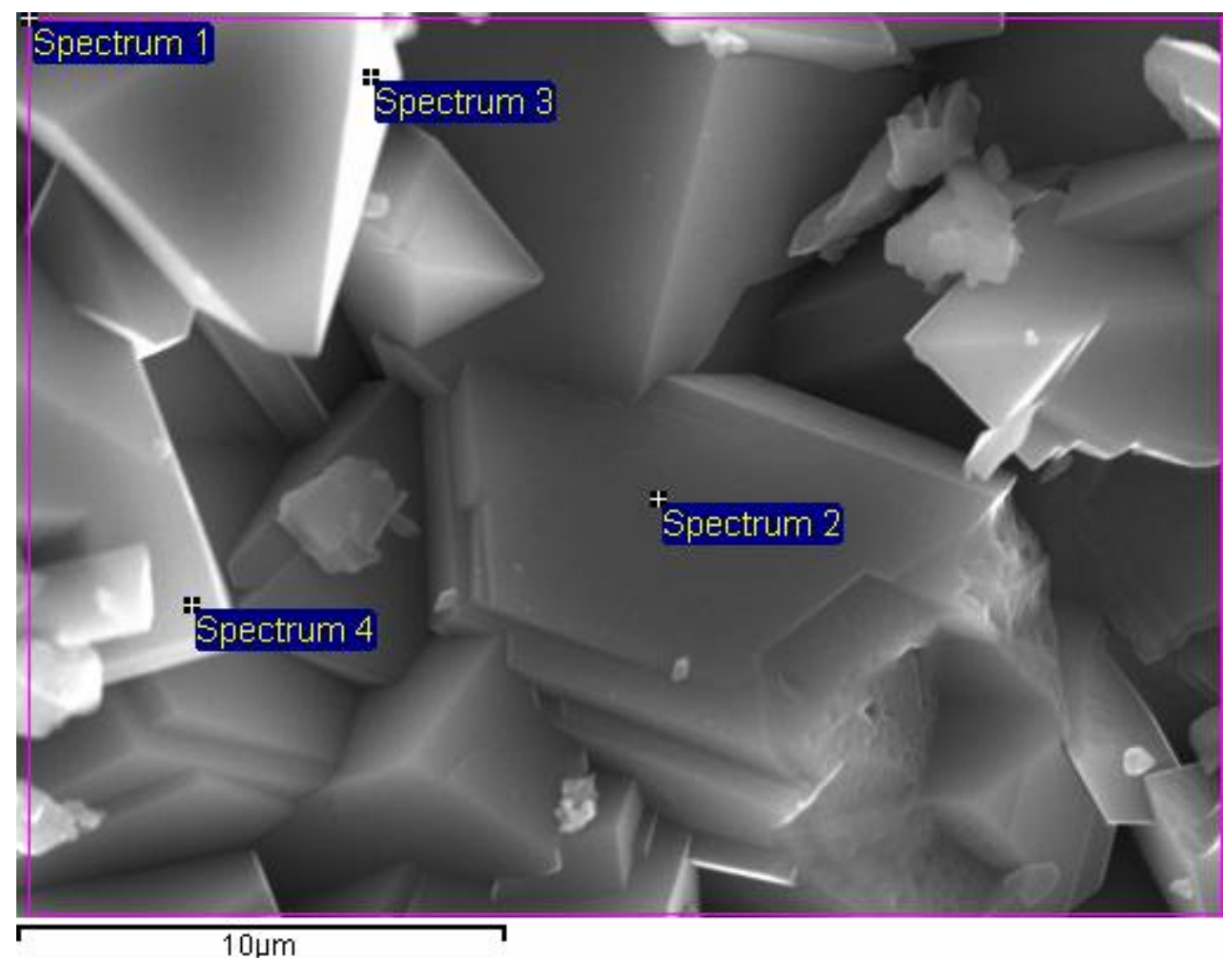

Spectrum1: EDX Analysis of the marked area of the sample.

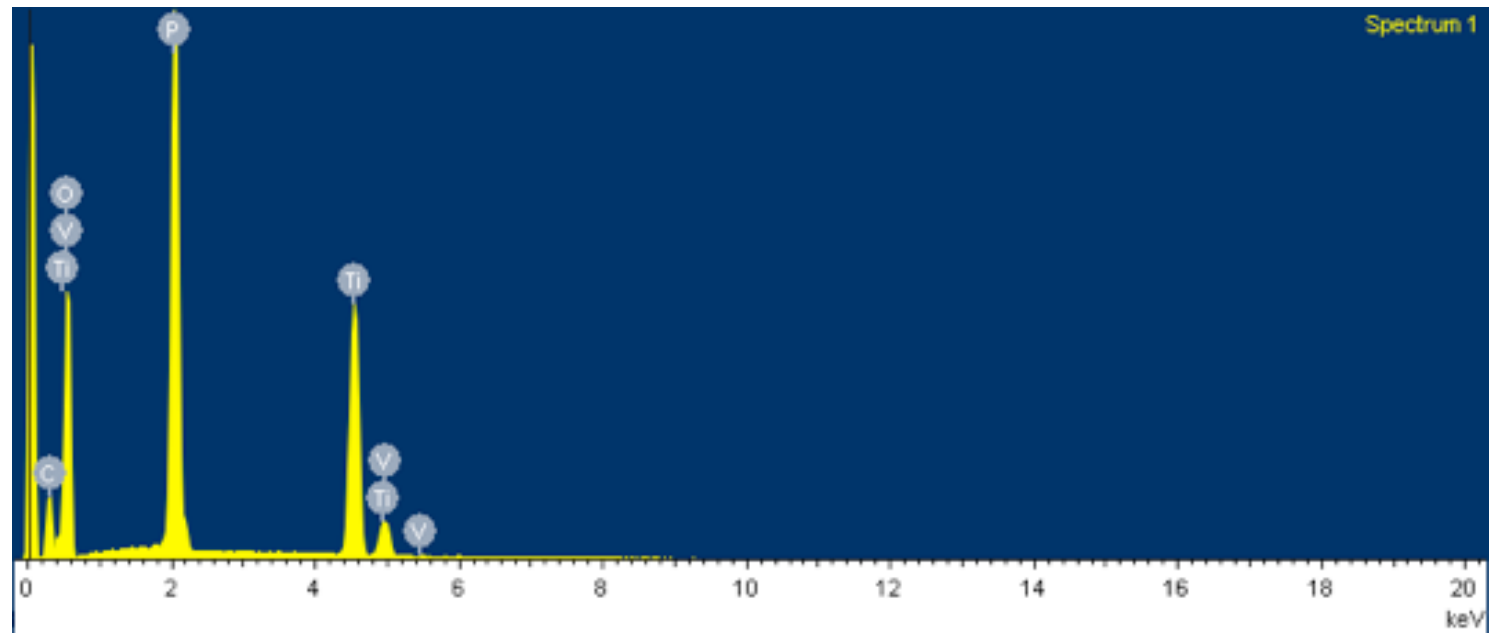


Spectrum2: EDX analysis of the cross-marked point (2) of the sample.

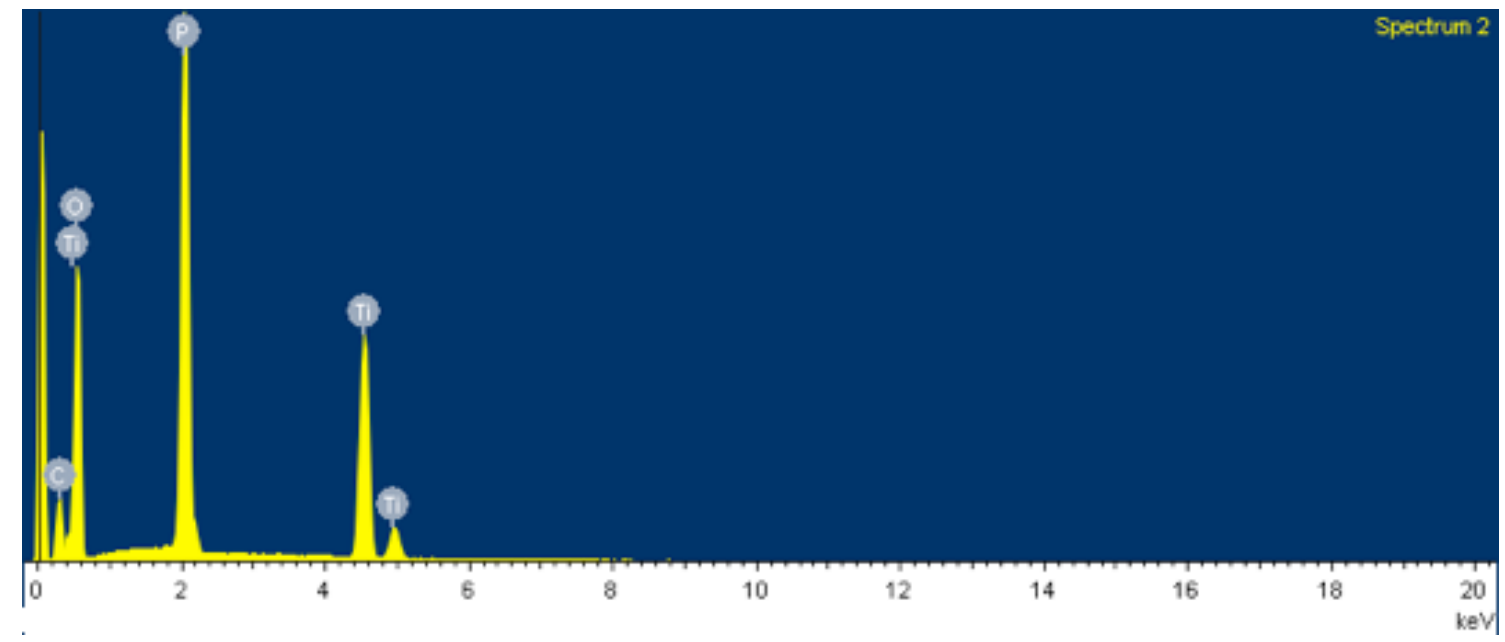

Spectrum3: EDX analysis of the cross-marked point (3) of the sample.

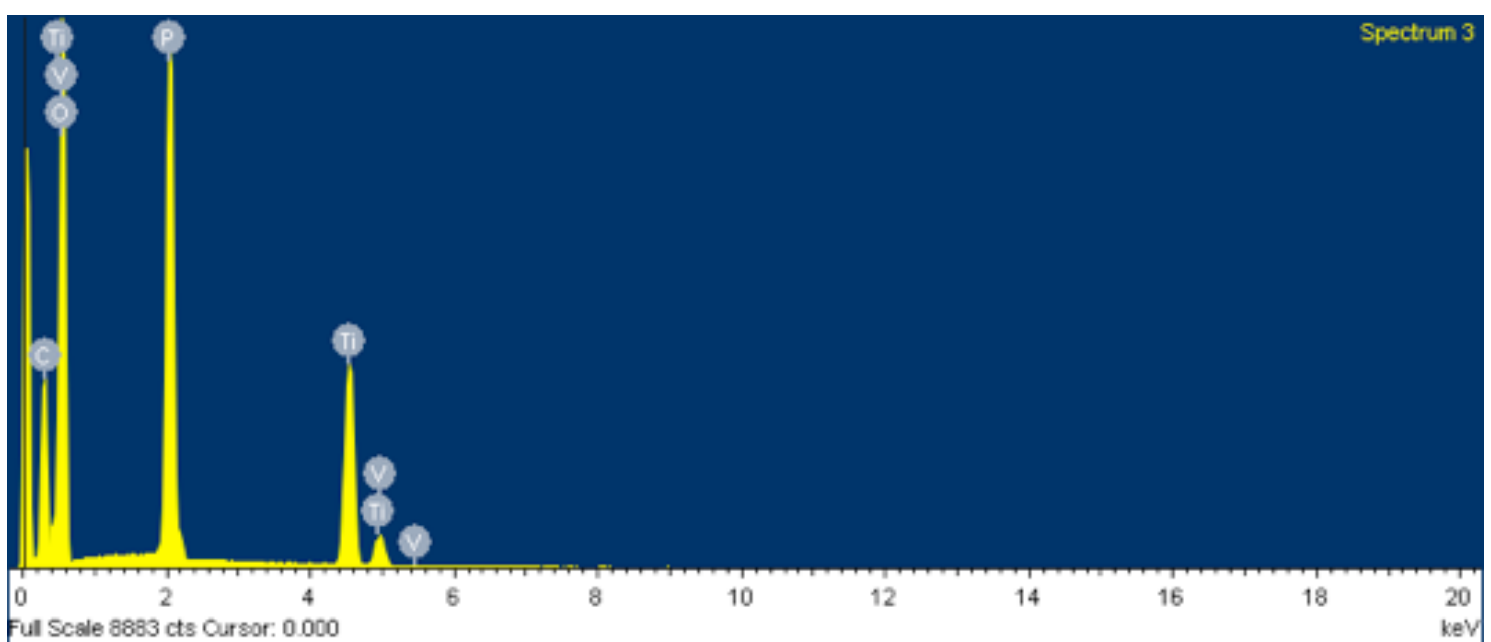

Spectrum3: EDX Analysis of the cross-marked point (4) of the sample.

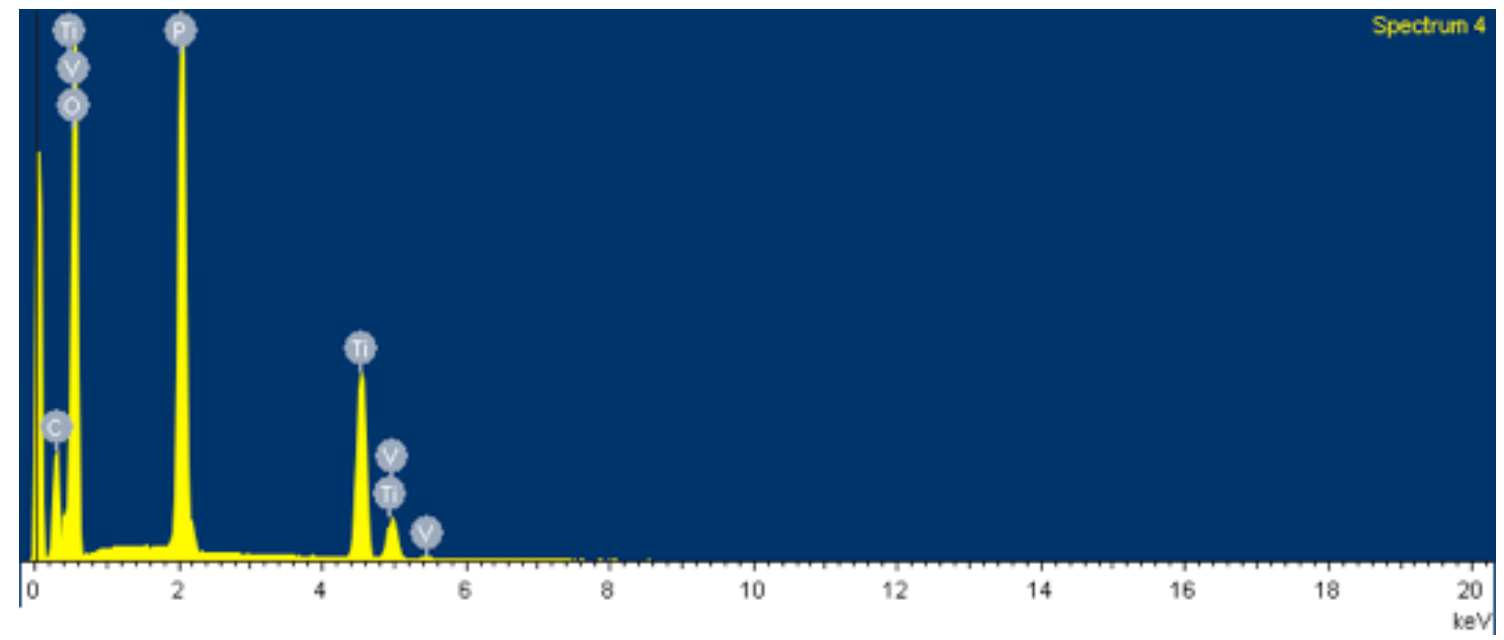

One of the interesting properties introduced by the presence of $\mathrm{Ti}^{3+}$ ions is a broad absorption band in the visible peaking at around $550 \mathrm{~nm}$. Figure 6.4. shows the 
diffuse reflectance $\mathrm{UV}$-Vis absorption spectra for $\left(\mathrm{Ti}^{3+} / \mathrm{Ti}^{4+}\right) \mathrm{P}$ containing various vanadium doping levels (samples M4-M7).

As can bee seen there, the general effect of vanadium doping is a decrease in the intensity of the visible band corresponding to $\mathrm{Ti}^{3+}$. This could suggest that vanadium is replacing preferentially this type of $\mathrm{Ti}^{3+}$ ions. Figure 6.4. also includes for the sake of comparison the absorption spectra of $\left(\mathrm{Ti}^{3+}\right) \mathrm{TiO}_{2}$ rutile (M8). According to the literature, ${ }^{[21]}$ the main absorption band corresponding to the direct band gap transition has an onset wavelength of about $410 \mathrm{~nm}$, considerably shifted with respect to the more intense band of M2 that absorbs below $330 \mathrm{~nm}$ deep in the UV region. The presence of some $\mathrm{Ti}^{3+}$ ions doping the $\mathrm{TiO}_{2}$ rutile is also reflected by a weak absorption band in the visible (see inset of Figure 6.4.) that is responsible for the blue appearance of M8, but comparatively is of much less intensity than the visible bands recorded for M2-M7 samples.

Figure 6.4. Diffuse reflectance UV-Vis absorption spectra (plotted as the KubelkaMunk function of the remittance (R)) of the V-doped $\left(\mathrm{Ti}^{3+} / \mathrm{Ti}^{4+}\right) \mathrm{P}(\mathrm{M} 2-\mathrm{M} 7)$ and $\left(\mathrm{Ti}^{3+}\right) \mathrm{TiO}_{2}(\mathrm{M} 8)$ samples. The dashed line corresponds to the emission spectrum of the visible-light LED employed as irradiation source plotted in arbitrary units (see chapter 8.2. for complete spectra). Inset (a) Magnification of the of the M8 spectra (b) Photo of the materials M5 (left) and M8 (right).

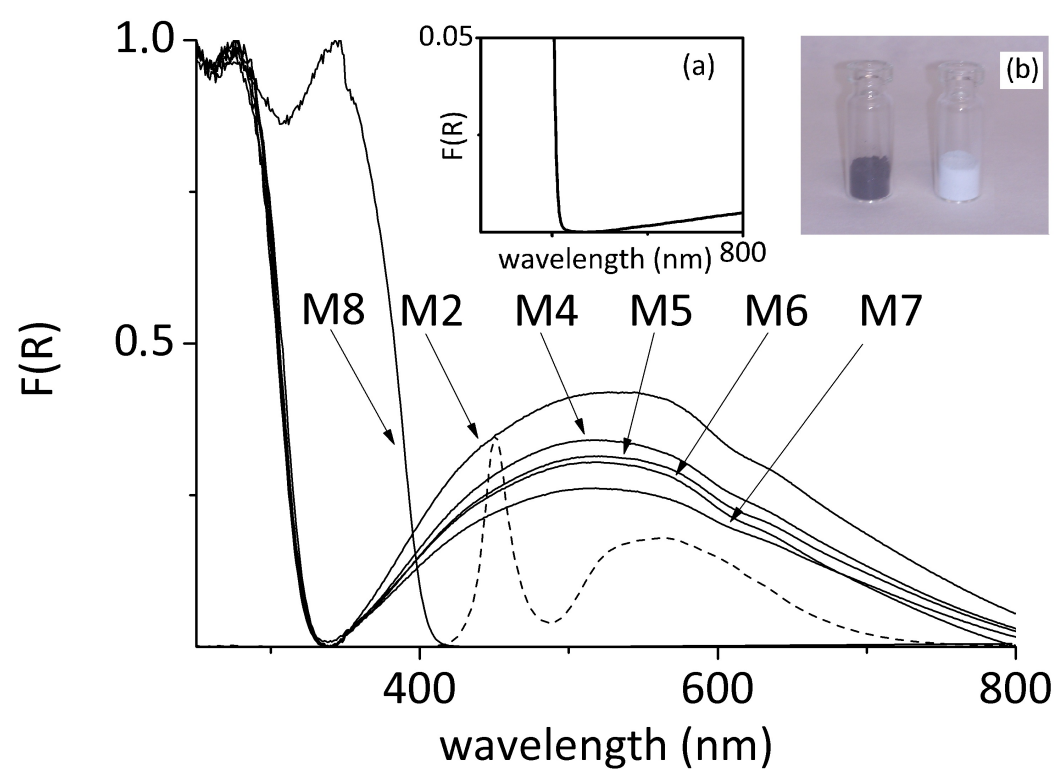




\subsubsection{Photocatalytic hydrogen generation}

As commented in the introduction, the present is aimed at showing the photocatalytic activity of mixed-valence $\left(\mathrm{Ti}^{3+} / \mathrm{Ti}^{4+}\right) \mathrm{P}$ and the influence of $\mathrm{V}$-doping. For this purpose we selected hydrogen generation from water-methanol mixtures as a test reaction to evaluate the photocatalytic efficiency of these materials, particularly in comparison with the activity of $\left(\mathrm{Ti}^{3+}\right) \mathrm{TiO}_{2}$ rutile that can be considered as a reference photocatalyst.

Preliminary tests show that irradiation of M1 with visible light in pure water did not show any hydrogen evolution. As expected in view of the literature data $\mathrm{TiO}_{2}$ is also devoid of any visible light photocatalytic activity for this reaction. Even the $\left(\mathrm{Ti}^{3+}\right) \mathrm{TiO}_{2}$ sample containing $\mathrm{Ti}^{3+}$ ions as dopant exhibits a negligible photocatalytic activity under these conditions. This lack of activity can be understood considering that the crystal phase of $\left(\mathrm{Ti}^{3+}\right) \mathrm{TiO}_{2}$ is rutile that has been frequently considered much less active than anatase or than the combination of anatase with a low proportion of rutile (case of P25). In contrast, samples M1-3 exhibit a significant photocatalytic activity for $\mathrm{H}_{2}$ generation under these conditions using visible-light from a LED as irradiation source. To put the catalytic activity in the context it has to be commented that samples M1-M3 do not contain platinum or any other noble metal that is known to act as co-catalyst increasing the activity for hydrogen generation. In spite of the increase of photocatalytic activity, the presence of noble metals constitutes a severe limitation for the wide application of a photocatalyst for hydrogen production due to their high cost. According to Figure 6.5. the most efficient photocatalyst both at 6 and $24 \mathrm{~h}$ with visible light irradiation is the one obtained after two days of crystallization (M2). It is worth commenting that crystallinity does not appear to be a key factor in the present case since sample M3 that is less crystalline that M1 or M2 exhibit also a notable catalytic activity, higher than of sample M1.

Based on this data we selected 2 days as the optimal crystallization time for the preparation of other $\left(\mathrm{Ti}^{3+} / \mathrm{Ti}^{4+}\right) \mathrm{P}$ samples and we performed a study of the influence of vanadium doping on the photocatalytic activity. As commented in the introduction, one of the purposes of the present chapter is to show the large flexibility that phosphates as materials offers with regard to the possibility to enhance the photocatalytic activity by doping. 
Figure 6.5. Photocatalytic hydrogen production from water-methanol mixtures using M1-M3 samples as photocatalysts under visible-light irradiation. Experimental conditions: $25 \mathrm{~mL} \mathrm{CH} \mathrm{CH}_{3} \mathrm{OH} / \mathrm{H}_{2} \mathrm{O}$ (1:1), $25 \mathrm{mg}$ of photocatalyst, reaction temperature $37-40{ }^{\circ} \mathrm{C}$. Inset (a) Hydrogen production at $24 \mathrm{~h}$ inset (b) Initial reaction rate calculated at $1 \mathrm{~h}$ irradiation.

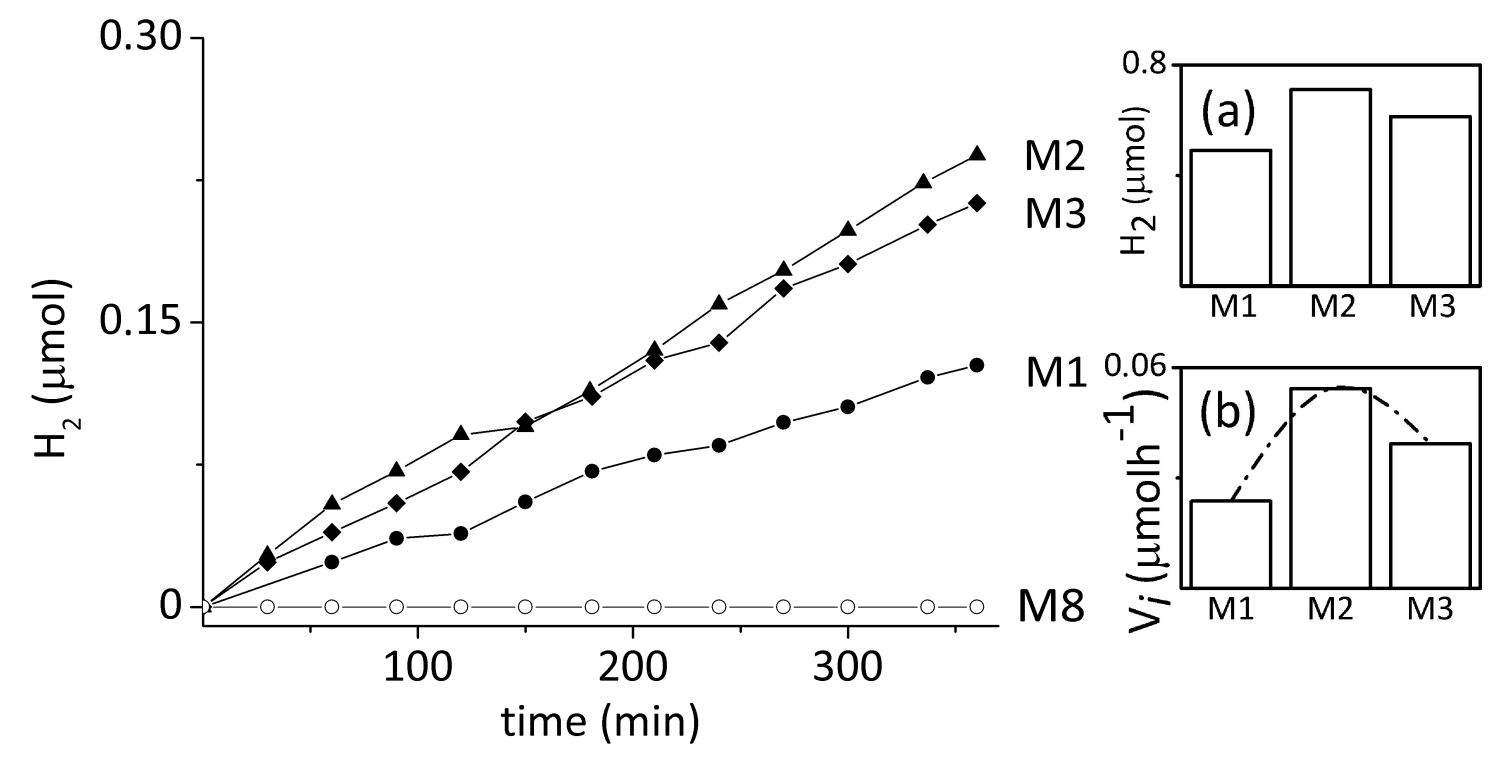

For this reason the visible light photocatalytic activity of V-doped samples M4-M7 was also tested under the same conditions. The results are listed in Table 6.1. and presented in Figure 6.6. As can be seen in this Figure, the photocatalytic activity of the undoped samples M2 increases, up to a factor of 5, due to the presence of vanadium as dopant element. If the initial reaction rate for visible-light hydrogen generation is plotted versus $\mathrm{V}$ concentration a bell shape curve is obtained, indicating that there is an optimal $\mathrm{V}$ content of about $0.5 \mathrm{wt} \%$. In precedents in the literature studying the influence of doping on titanium dioxide and other semiconductors it has also been frequently observed that the presence of doping enhances the photocatalytic activity up to an optimal loading and then higher doping levels are detrimental for the photocatalytic activity.[17] This negative effect of increasing doping loadings has been attributed to an enhancement of charge recombination at the dopant elements for high dopant contents. 
Figure 6.6. Photocatalytic hydrogen production from water-methanol mixtures using M2, M4-M7 samples as photocatalysts under visible-light irradiation. Experimental conditions: $25 \mathrm{~mL} \mathrm{CH} \mathrm{CH}_{3} \mathrm{OH} / \mathrm{H}_{2} \mathrm{O}$ (1:1), $25 \mathrm{mg}$ of photocatalyst, reaction temperature $37-40{ }^{\circ} \mathrm{C}$. Inset (a) Hydrogen production at $24 \mathrm{~h}$ (b) Initial velocity calculated at $1 \mathrm{~h}$ irradiation.

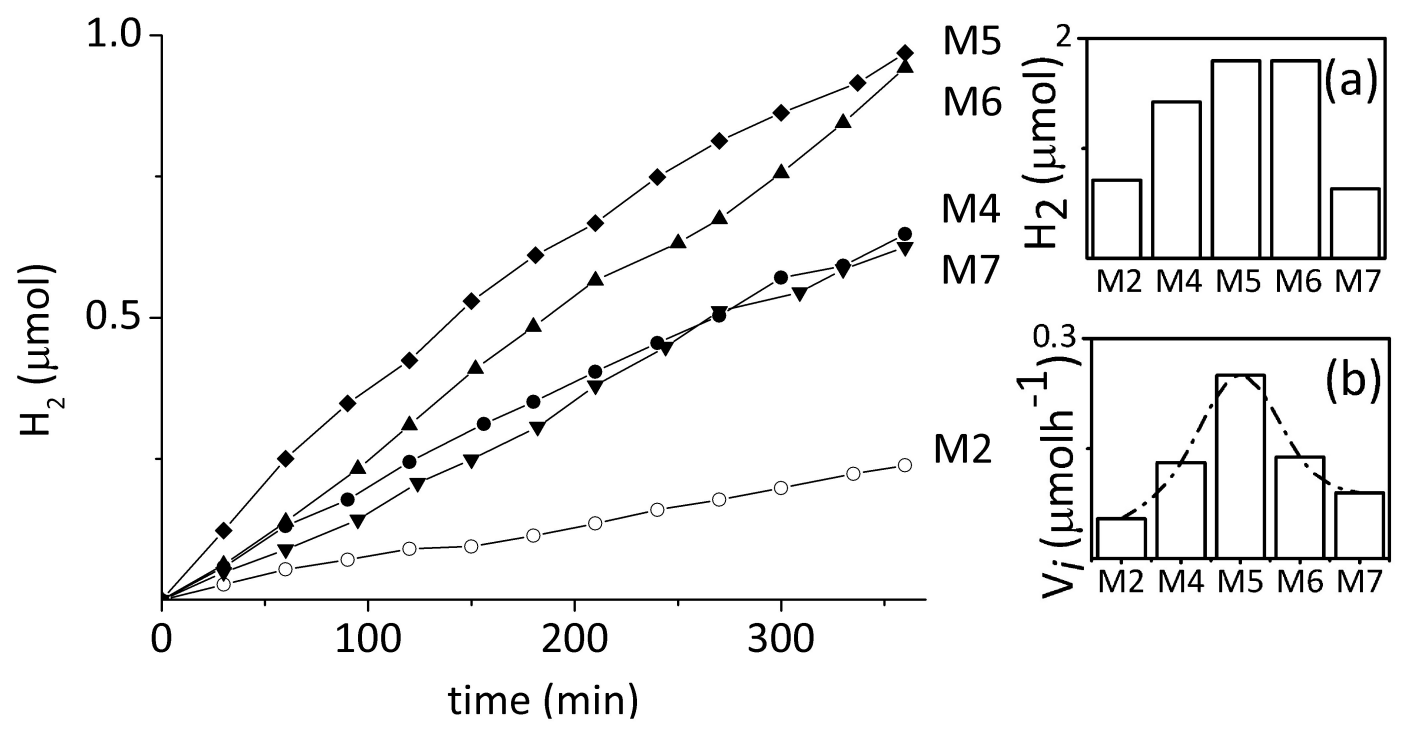

Table 6.1. Sample codes, composition and photocatalytic activity data for the mixed-valence $\left(\mathrm{Ti}^{3+} / \mathrm{Ti}^{4+}\right) \mathrm{P}$ samples under study ${ }^{(\mathrm{a})}$.

\begin{tabular}{|c|c|c|c|c|c|}
\hline Sample & $\begin{array}{l}\text { Metal } \\
\text { (wt } \% \\
\text { V }\end{array}$ & $\begin{array}{l}\text { Composition } \\
\text { Ti }\end{array}$ & $\begin{array}{l}\mathrm{H}_{2} \\
(\mu \mathrm{mc} \\
6 \mathrm{~h}\end{array}$ & $\begin{array}{l}\text { production } \\
24 \mathrm{~h}\end{array}$ & $\begin{array}{ll}\text { Initial } & \text { rate } \\
\left(\mu \mathrm{mol} / \mathrm{h} \cdot \mathrm{g}_{\mathrm{Ti}}\right) & \end{array}$ \\
\hline $\mathrm{M} 1^{(\mathrm{b})}$ & - & 100 & 0.12 & 0.49 & 3.83 \\
\hline M2 & - & 100 & 0.23 & 0.71 & 9.57 \\
\hline M3 & - & 100 & 0.21 & 0.61 & 5.73 \\
\hline $\mathrm{M} 4(\mathrm{c})$ & 0.24 & 99.75 & 0.64 & 1.42 & 24.90 \\
\hline M5 & 0.54 & 99.46 & 0.96 & 1.79 & 47.89 \\
\hline M6 & 0.96 & 99.04 & 0.94 & 1.79 & 24.90 \\
\hline M7 & 2.32 & 97.68 & 0.62 & 0.63 & 15.32 \\
\hline $\mathrm{M}^{(\mathrm{d})}$ & - & 100 & - & 0.02 & 0.055 \\
\hline M9(e) & 0.48 & 99.52 & 0.14 & 0.31 & 9.21 \\
\hline M10 & 2.1 & 97.9 & 0.14 & 0.2 & 8.98 \\
\hline
\end{tabular}


(a) Irradiation conditions: $25 \mathrm{ml} \mathrm{CH} 3 \mathrm{OH}_{3} \mathrm{H}_{2} \mathrm{O}$ (1:1) $25 \mathrm{mg}$ catalyst reaction temperature $37-40{ }^{\circ} \mathrm{C}$. (b) Empirical formula of $\left(\mathrm{Ti}^{3+} / \mathrm{Ti}^{4+}\right) \mathrm{P}$ compounds (M1-M7) is $\mathrm{Ti}_{(2-\mathrm{x})} \mathrm{V}_{\mathrm{x}}\left(\mathrm{PO}_{4}\right)\left(\mathrm{HPO}_{4}\right)_{2}(\mathrm{H} 2 \mathrm{O})_{2} \cdot\left(\mathrm{NH}_{2} \mathrm{CH}_{2} \mathrm{CH}_{2} \mathrm{CH}_{2} \mathrm{NH}_{2}\right)_{0.5}$.(c) The amount of $\mathrm{V}$ present in the material was determined by ICP (d) M8 corresponds to $\mathrm{Ti}^{3+}$-doped $\mathrm{TiO}_{2}$ rutile. (e) M9 and M10 samples consist in a mechanical mixture of M2 and $\mathrm{V}_{2} \mathrm{O}_{5}$.

As commented when describing the XRD patterns, crystallinity of doped samples and, particularly, M5 was based on the coincidence of the diffraction peaks with that of undoped samples. However, it could be possible that the low percentage of vanadium could be present, at least partially, as an independent or separated phase.

In order to rule out conclusively that the enhancement the photocatalytic activity could derive from such a mixture of different materials in different phases, we also evaluated the photocatalytic activity for $\mathrm{H}_{2}$ generation with visible light of two $\mathrm{V}_{2} \mathrm{O}_{5} /\left(\mathrm{Ti}^{3+} / \mathrm{Ti}^{4+}\right) \mathrm{P}$ mixtures (M9 and M10) and the result are also included in Table 6.1. and Figure 6.7. As can bee seen there, the photocatalytic activity and the initial rate of the $\mathrm{V}_{2} \mathrm{O}_{5} /\left(\mathrm{Ti}^{3+} / \mathrm{Ti}^{4+}\right) \mathrm{P}$ is very similar of that of sample $\mathrm{M} 2$, and much lower than the photocatalytic series of sample M4-M7. These results are not surprising and can be interpreted as if the photocatalytic activity of the mixture derives from $\mathrm{M} 2$ the predominant component and as if the small portion of $\mathrm{V}_{2} \mathrm{O}_{5}$ does not make any contribution to the photocatalytic hydrogen generation. This poor catalytic activity exhibited by the $\mathrm{V}_{2} \mathrm{O}_{5} /\left(\mathrm{Ti}^{3+} / \mathrm{Ti}^{4+}\right) \mathrm{P}$ mixtures that is similar to that of pure M2 speaks out against that the photocatalytic data shown in Figure 6.6. and Figure 6.7. could be due to the presence of $\mathrm{V}_{2} \mathrm{O}_{5}$ as independent phase and provide additional indirect support to the occurrence of doping in $\left(\mathrm{Ti}^{3+} / \mathrm{Ti}^{4+}\right) \mathrm{P}$ materials. 
Figure 6.7. Photocatalytic hydrogen production from water-methanol mixtures using M2, M9 and M10 samples as photocatalyst under visible-light irradiation. Experimental conditions: $25 \mathrm{~mL} \mathrm{CH}_{3} \mathrm{OH} / \mathrm{H}_{2} \mathrm{O}$ (1:1), $25 \mathrm{mg}$ of photocatalyst, reaction temperature $37-40^{\circ} \mathrm{C}$.

Inset (a) Hydrogen production at $24 \mathrm{~h}$ inset (b) Initial reaction rate calculated at $1 \mathrm{~h}$ irradiation.

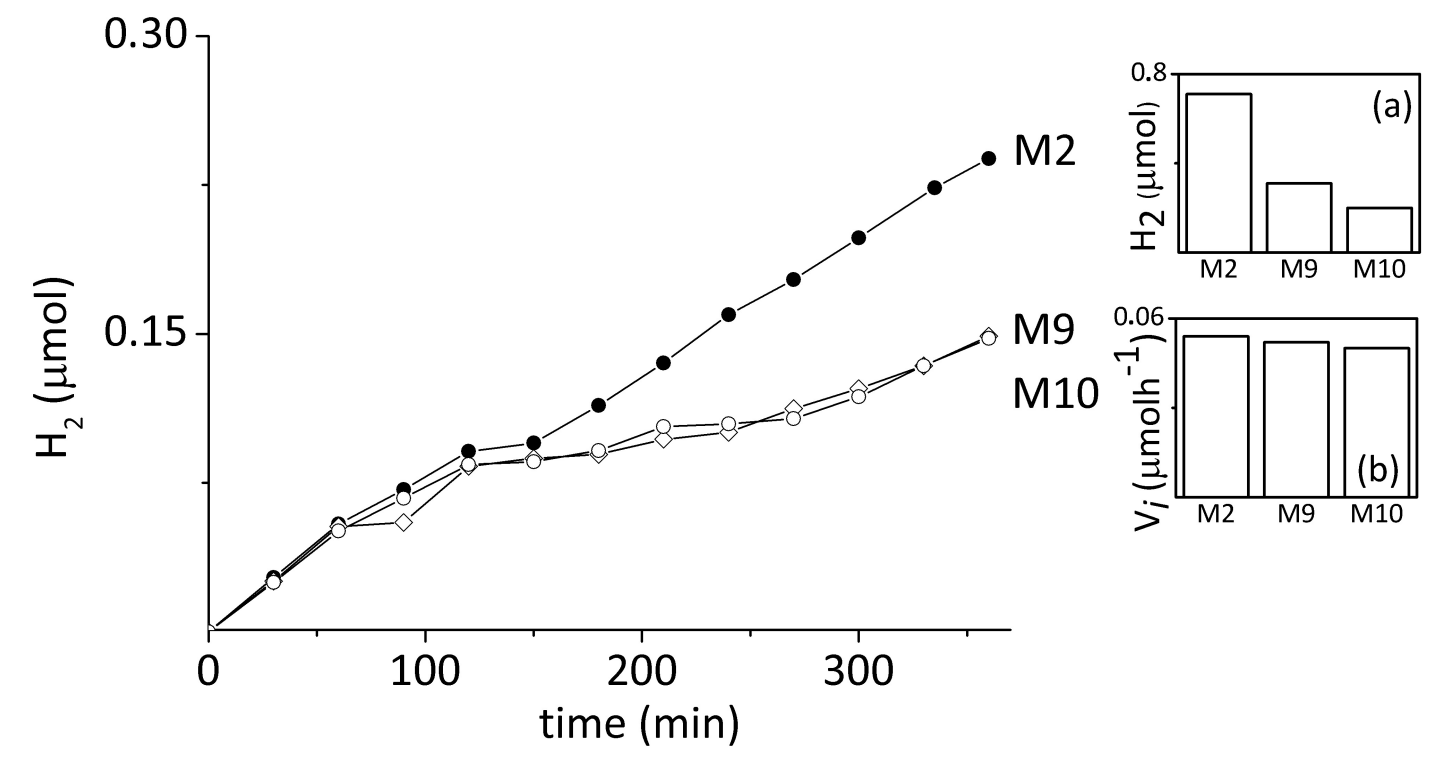

The photocatalytic activity for hydrogen generation from water methanol mixture was completed by evaluation of the activity of these materials under simulated sunlight irradiation. These conditions in which simulated sunlight is used as excitation source are closer the conditions for possible commercial application of the material. As can be seen in Figure 6.8. the order of the photocatalytic activity of M2, M4-M7 samples is the same as the one observed for visible light irradiation using a LED. The somewhat higher hydrogen amount generated using solar simulator is difficult to correlate with the visible-light photocatalytic activity by LED due to the different power and spectra of the two light sources. The main differences upon irradiation with visible or simulated sunlight is photocatalytic activity of M8 rutile that is devoid of photocatalytic activity upon LED irradiation, but exhibits a photocatalytic activity higher than M2, but still lower than V-doped $\left(\mathrm{Ti}^{3+} / \mathrm{Ti}^{4+}\right) \mathrm{P}$. 
Figure 6.8. Photocatalytic hydrogen production from water-methanol mixtures using M2, M4-M7 samples as photocatalysts under simulated sunlight. Experimental conditions: $25 \mathrm{~mL} \mathrm{CH} \mathrm{CH}_{3} \mathrm{OH} / \mathrm{H}_{2} \mathrm{O}$ (1:1), $25 \mathrm{mg}$ of photocatalyst, reaction temperature $37-40{ }^{\circ} \mathrm{C}$. Inset (a) Initial velocity calculated at $1 \mathrm{~h}$ irradiation.

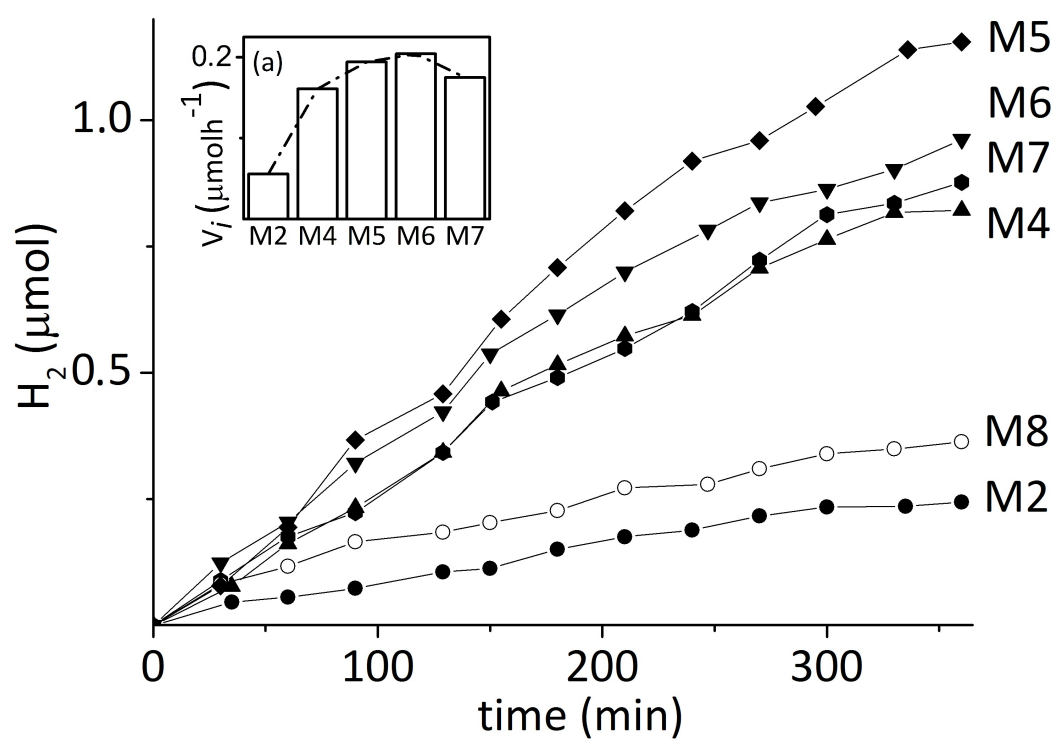

The issue of the long term stability of vanadium doped $\left(\mathrm{Ti}^{3+} / \mathrm{Ti}^{4+}\right) \mathrm{P}$ as photocatalyst was addressed by performing a prolonged irradiation experiments for one week using ten times larger volumes and photocatalyst. It was observed that during such a comparatively long time the amount of hydrogen grows linearly during the whole period of time, as show in Figure 6.9.

Furthermore, at the end of irradiation the used $\left(\mathrm{Ti}^{3+} / \mathrm{Ti}^{4+}\right) \mathrm{P}$ was recovered and characterized by UV-Vis spectroscopy and XRD. No significant changes with respect to the initial fresh sample was observed for the exhaustively used material, indicating the stability of the $\left(\mathrm{Ti}^{3+} / \mathrm{Ti}^{4+}\right) \mathrm{P}$ during the operation of the photocatalytic reaction. 
Figure 6.9. Long-term photocatalytic hydrogen production from water-methanol mixtures using M5 sample as photocatalyst under visible-light irradiation. Experimental conditions: $250 \mathrm{~mL} \mathrm{CH} \mathrm{CH}_{3} \mathrm{OH} / \mathrm{H}_{2} \mathrm{O}$ (1:1), $250 \mathrm{mg}$ of photocatalyst, reaction temperature $38{ }^{\circ} \mathrm{C}$. Inset (a) Comparison of diffuse reflectance UV-Vis absorption spectra (plotted as the Kubelka-Munk function of the remittance (R)) of the fresh $\mathrm{V}$-doped $\left(\mathrm{Ti}^{3+} / \mathrm{Ti}^{4+}\right) \mathrm{P}$ sample (M5) and the same sample used as photocatalyst during 7 days (M5').

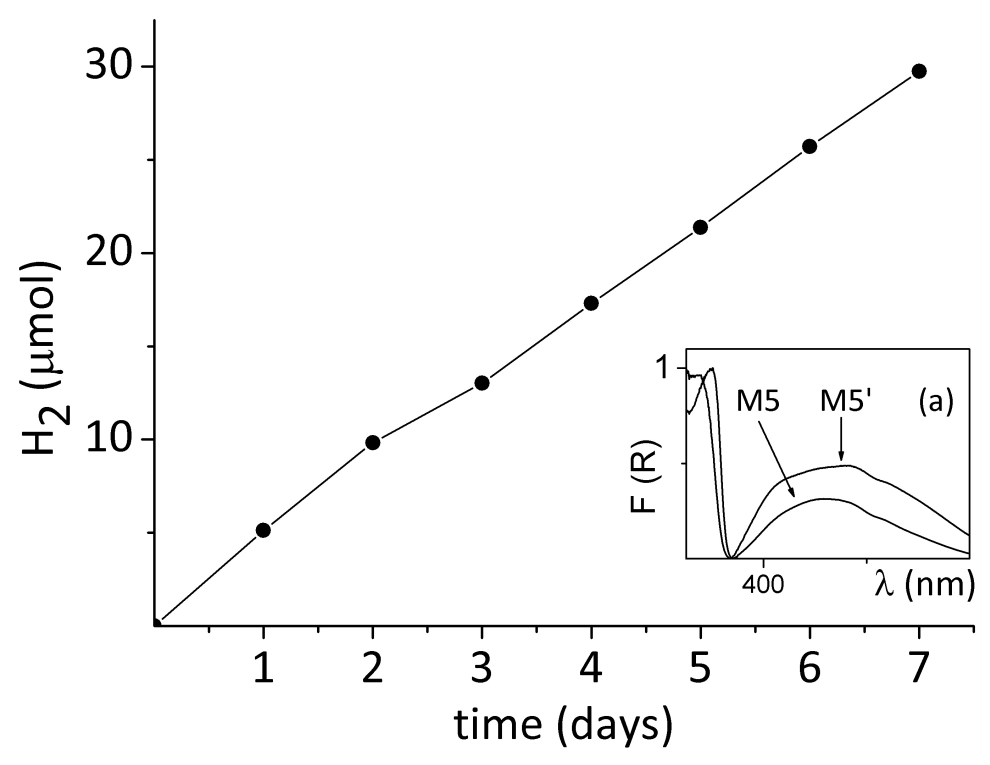

\subsubsection{Characterization of $\left(\mathrm{Ti}^{3+} / \mathrm{Ti}^{4+}\right) P$ as semiconductor}

After having observed photocatalytic activity for mixed valence $\left(\mathrm{Ti}^{3+} / \mathrm{Ti}^{4+}\right) \mathrm{P}$ samples and in order to support on solid grounds the behaviour of these materials as semiconductor, we performed photocurrent measurements to determine the flatband potential of electrons in the conduction band of this material as well as carried out the transient absorption spectroscopy study to detect the charge separation state and to monitor the kinetics and the quenching behaviour of this transient state. By exciting the samples with monochromatic light at $355 \mathrm{~nm}$ and measuring the photocurrent as a function of the applied voltage, a flat band potential for the conduction band of $-0.1 \mathrm{eV}$ versus NHE was determined for M2 sample (see chapter 8). 
This potential is just about the one required for hydrogen generation, it is similar to the potential of electrons of the conduction band of $\mathrm{TiO}_{2}$. Similar value $(-0.13$ $\mathrm{eV}$ ) was determined for the conduction band of $\left(\mathrm{Ti}^{3+} / \mathrm{Ti}^{4+}\right) \mathrm{P}$ using impedance spectroscopy that also gives an estimation of the charge carrier density for $\left(\mathrm{Ti}^{3+} / \mathrm{Ti}^{4+}\right) \mathrm{P}$ as $2 \cdot 10^{20}$ carriers $/ \mathrm{cm}^{3}$ (see chapter 8 ). It should be, however, commented that the measurements of impedance spectroscopy were complicate by the fact that it is not possible to sinter the film of $\left(\mathrm{Ti}^{3+} / \mathrm{Ti}^{4+}\right) \mathrm{P}$ on the FTO electrode, due to the thermal instability of $\left(\mathrm{Ti}^{3+} / \mathrm{Ti}^{4+}\right) \mathrm{P}$ that it is known to decompose a temperature higher of $150{ }^{\circ} \mathrm{C}$. In the present chapter, the films of $\left(\mathrm{Ti}^{3+} / \mathrm{Ti}^{4+}\right) \mathrm{P}$ were prepared by prolonged heating of the $\left(\mathrm{Ti}^{3+} / \mathrm{Ti}^{4+}\right) \mathrm{P}$ powder on FTO at $80{ }^{\circ} \mathrm{C}$. Transient absorption spectra were recorded upon excitation in the UV $(266 \mathrm{~nm})$ or in the visible $(532 \mathrm{~nm})$ region.

As we will show later, although the transient spectra recorded at these two excitation wavelengths are very broad, the remarkable differences in lifetimes and temporal profile of the signal indicate that the location of the electrons and holes are different depending on the excitation wavelength. We noticed that according to the absorption spectra of the samples these two irradiation wavelengths may correspond to excitation of two different chromophores in the $\left(\mathrm{Ti}^{3+} / \mathrm{Ti}^{4+}\right) \mathrm{P}$ samples, presumably irradiation to the $\left(\mathrm{Ti}^{4+}\right) / \mathrm{P}$ ion chromophore or to the $\left(\mathrm{Ti}^{3+}\right) / \mathrm{P}$ chromophore for the 266 and $532 \mathrm{~nm}$, respectively. For the sake of comparison we have also included in the transient absorption spectroscopic study a sample of $\left(\mathrm{Ti}^{4+}\right) \mathrm{P} .{ }^{[26]}$

Upon $266 \mathrm{~nm}$ laser excitation of $\left(\mathrm{Ti}^{4+}\right) \mathrm{P}$ or samples M2, M4-M7 the same transient spectra consisting in a continuous absorption with similar absorptivity in all the spectral window was recorded. As an example Figure 6.10. shows a selected transient absorption spectrum recorded for M2. The temporal profile of the signal for each sample was coincident in all the spectral range (see for instance the inset of Figure 6.10.). These temporal profiles consist in two regimes, one decaying very fast almost in the limit of our nanosecond detection system and accounting between 90 and $70 \%$ of the total intensity, a second time regime spanning from $0.12 \mu \mathrm{s}$ to $10 \mu \mathrm{s}$. It is worth to comment that the transient spectra of these two regimes were identical (inset Figure 6.10.). 
The vanadium content of the sample has no influence on the transient spectra or temporal profile, but however, increases the intensity of the $\Delta \mathrm{J} / \mathrm{J}_{0}$ signal at zero time (data not shown). In order to give some evidence of the nature of the signal quenching experiments of the signal using oxygen (electron quencher) and ethanol (hole quencher) were performed.

Figure 6.10. Transient spectra recorder for an acetonitrile suspension of M2 upon $266 \mathrm{~nm}$ excitation recorded at 40,600 and $1730 \mu \mathrm{s}$. The inset shows transient signal recorded for a $\mathrm{N}_{2}$ purged acetonitrile suspension of M6 upon $266 \mathrm{~nm}$ excitation monitored at 400 (a) and 600 (b) nm.

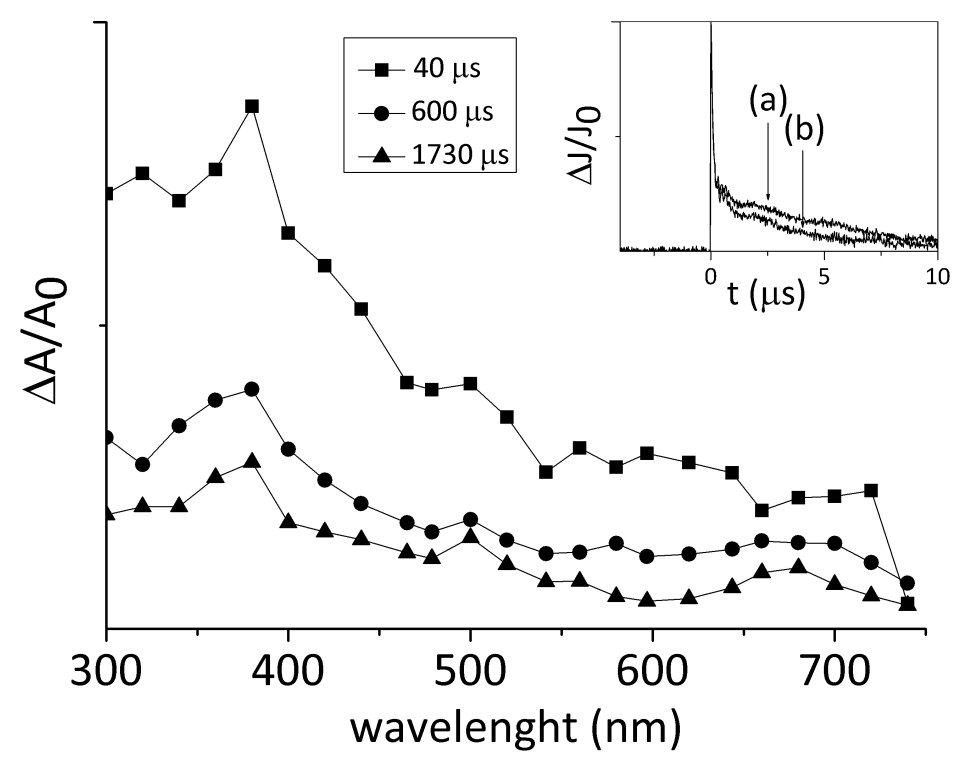

Again the presence of any of these quenchers did not influence the transient spectra, but had a notable influence on the temporal profile of the signal, particularly in the faster regime. Oxygen increases the top $\Delta \mathrm{J} / \mathrm{J}_{0}$ signal by almost a factor 2 for M2 and 1.5 for M5. In contrast ethanol decreases the top $\Delta \mathrm{J} / \mathrm{J}_{0}$ value in both cases, particularly, by a factor 1.5 for sample M5. This behavior is consistent with the transient absorption spectrum in the short life regime been dominated by holes, whose number will increase in presence of oxygen by decreasing their recombination with electrons that will be removed by oxygen. When ethanol is present the signal decreases due to the quenching of the holes being monitored. 
In the long time regime the influence of the presence of quencher is much smaller particularly for V-doped samples. This suggests that electrons and holes had been trapped at certain sites in the materials and are less available to interact with quenchers. In fact a careful analysis of temporal profiles shows that the fast decay is followed by a minor growth of the signal that can be attributed to the relocation of the charge carriers in the materials. Importantly the presence of vanadium at loading between $0.5-1 \mathrm{wt} \%$ is reflected by a highest transient signal intensity, suggesting that the vanadium is also involved in the generation of electrons and holes.

The $532 \mathrm{~nm}$ laser excitation has some differences with respect to $266 \mathrm{~nm}$. The first one is that $\left(\mathrm{Ti}^{4+}\right) \mathrm{P}$ does not exhibit any transient signal in accordance with the lack of excitation of this white powder that has not absorption band in the visible. In contrast samples M2, M4-M7 exhibit transients whose spectra were similar for all samples consisting in a broad absorption in all the spectroscopic range with an increase in the absorbance from $450 \mathrm{~nm}$ to $300 \mathrm{~nm}$. As an example Figure 6.11. shows the transient spectra recorded for M2-M5. The temporal profile of the signal was also coincident in the whole spectral range and was similar for sample M2, M4-M7, the most remarkable fact is now their extremely long lifetime as the inset in Figure 6.11. shows. As in case $266 \mathrm{~nm}$ excitation, also for $532 \mathrm{~nm}$ the temporal profiles have two regimes, one decaying very fast in a few $\mu$ s that correspond between 50 and $20 \%$ of the total signal and a second regime having a very long lived signal, whose decay is not complete even four ms after the lase pulse. We attribute this extremely long life of the transient to the relocation of the charges in certain ions of $\left(\mathrm{Ti}^{3+} / \mathrm{Ti}^{4+}\right) \mathrm{P}$ that should be significantly stable. It was observed, however, that the presence of oxygen and ethanol as electron and hole quencher, respectively, does not alter much the kinetics of the decay particularly in the long time regime. 
Figure 6.11. A) Transient spectra recorded for Ar-purged acetonitrile suspension of M2 upon $532 \mathrm{~nm}$ laser excitation monitorized at 40, 60 and $1730 \mu \mathrm{s}$. B) Transient signal recorded for an acetonitrile suspension of M2 upon $266 \mathrm{~nm}$ laser excitation after purging the sample with $\mathrm{Ar}$ (a), $\mathrm{O}_{2}$ (b) or $\mathrm{CH}_{3} \mathrm{CH}_{2} \mathrm{OH}$ (c). C) Transient signal recorded for an acetonitrile suspension of M2 upon $532 \mathrm{~nm}$ laser excitation after purging with $\mathrm{N}_{2}(\mathrm{a}), \mathrm{O}_{2}$ (b) or quenching with $\mathrm{CH}_{3} \mathrm{CH}_{2} \mathrm{OH}$ (c)
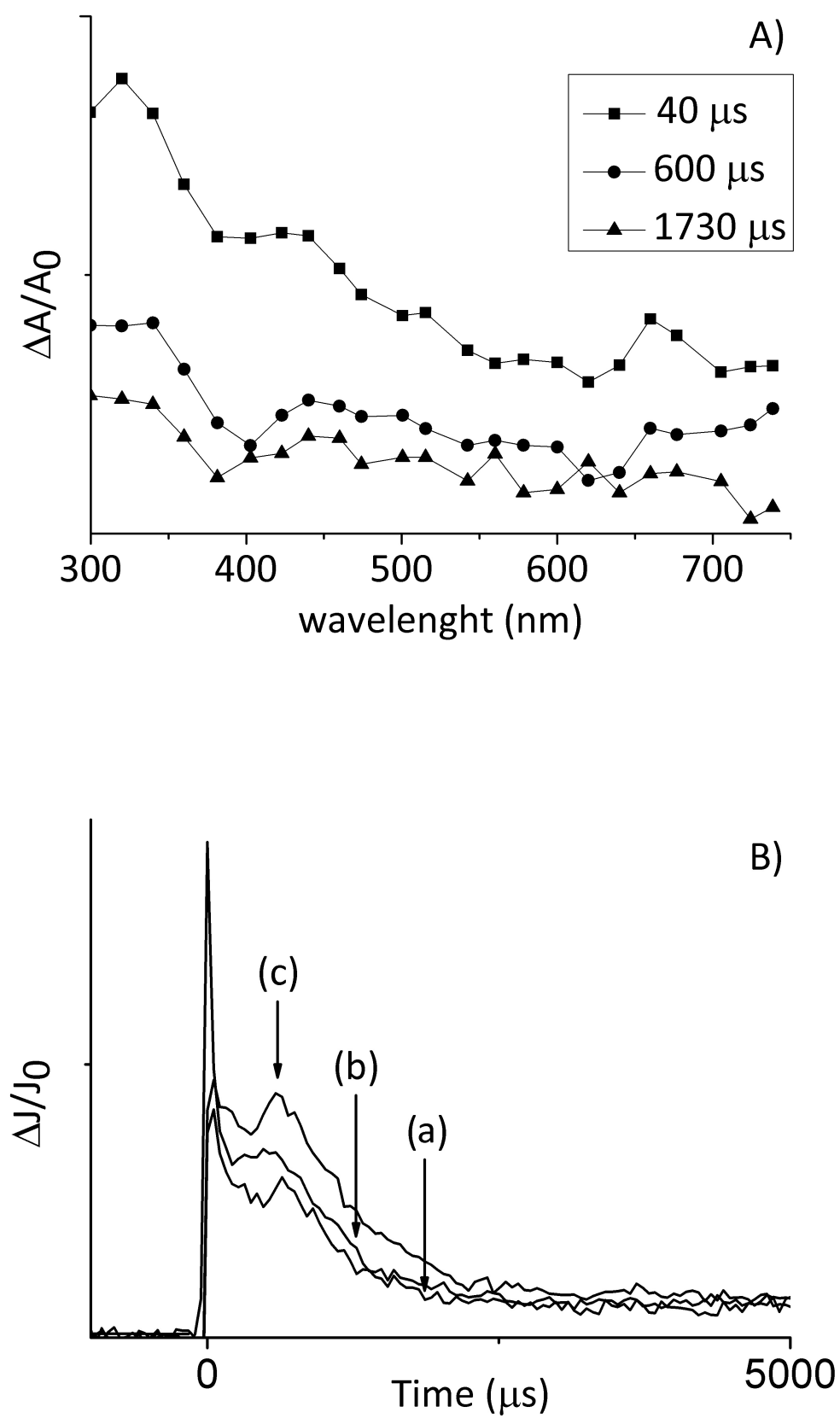


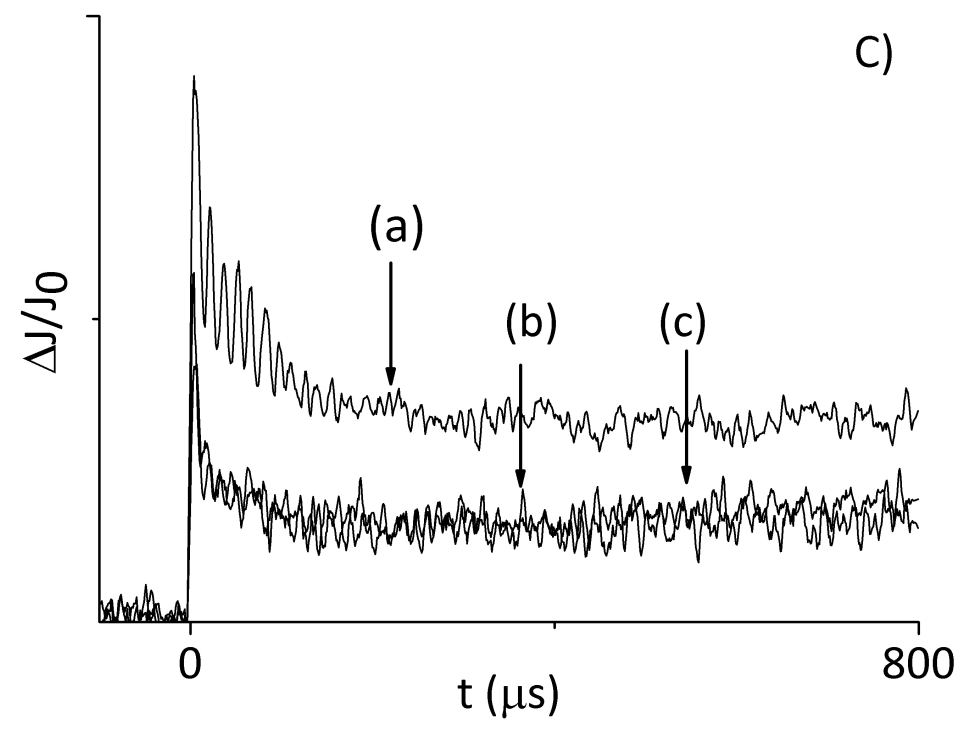

Only the intensity of the signals are altered by quenching, reflecting that the interaction with oxygen of ethanol is a static quenching or take places at much shorter time scales. As an example Figure 6.11. shows the quenching behaviour of M1-M6 with oxygen and ethanol showing that only the intensity of the signal is affected (Figure 6.11., part B and C).

The overall conclusion of transient spectroscopy is that there is a difference in the behaviour depending of the excitation wavelength, indicating that excitation at the $\left(\mathrm{Ti}^{4+}\right) \mathrm{P}$ band $\left(\lambda_{\max } 280 \mathrm{~nm}\right)$ or excitation at the (Ti $\left.{ }^{3+}\right) \mathrm{P}$ or (V)P leads to different location of electrons and holes. The influence of vanadium in the transient is increasing both at 266 and $532 \mathrm{~nm}$ wavelengths the charge separation without affecting the spectra and the kinetics of the signal. In other words, spectroscopic evidence from the time resolved study indicate that vanadium ions can be excited at both UV and Vis wavelengths leading to electrons and holes, the larger efficiency in the generation of electrons and holes being reflected in a relative higher signal intensity for V-doped samples. In contrast there is no evidence for the involvement of $\mathrm{V}$ ions in trapping of the charge carrier that should be reflected probably in variation in the transient spectra or in the kinetics of electron-hole recombination (variation in the temporal profile) had been obtained. In summary, its seam that vanadium ions increases the population of electrons and holes (higher signal intensity either at 266 or $532 \mathrm{~nm}$ ) without affecting to their relocation or their 
reactivity (same transient spectra as those shown in Figures 6.11. and 6.10. for M2 and same temporal profile of the signal). Finally quenching, particularly by methanol, that is employed in the photocatalytic hydrogen generation, has been observed only for the $266 \mathrm{~nm}$ excitation and for the short (submicrosecond) time regime. According to this data, an increase of the efficiency of the $\left(\mathrm{Ti}^{3+} / \mathrm{Ti}^{4+}\right) \mathrm{P}$ would be achieved if the charge carriers were more quenchable by methanol.

\subsection{Conclusions.}

In the present manuscript we have shown that mixed-valence $\mathrm{Ti}^{3+} / \mathrm{Ti}^{4+}$ phosphates, up to now ignored as photocatalysts, can be easily prepared and exhibit behavior as semiconductor, with a conduction band reduction potential and density of charge carriers similar to that of $\mathrm{TiO}_{2}$. The state of charge separation generated upon light absorption can be detected by nanosecond transient absorption spectroscopy and is characterized by a broad absorption band spanning the whole all window of UV and visible wavelengths. The decay of the signal has two time regimes, one that is predominant and disappears in $0.15 \mu \mathrm{s}$ and other that is about $20 \%$ and expand to $10 \mu \mathrm{s}$. Vanadium doping increases the intensity of the transient signal without changing the spectra of the temporal profile.

This mixed valence $\mathrm{Ti}^{3+} / \mathrm{Ti}^{4+}$ phosphate exhibits a notable photocatalytic activity for hydrogen generation with visible light that is much higher than that of analogous sample of $\mathrm{TiO}_{2}$ reaching the values of about $50 \mu \mathrm{molh}^{-1} \mathrm{~g}_{\mathrm{Ti}^{-1}}$ in the absence of platinum as cocatalyst. The visible light photocatalytic activity should derive from the visible light absorption introduced by the presence of $\mathrm{Ti}^{3+}$ ions. In addition we have shown that this type of phosphate can easily be doped with neighbour transition metals. In the present case, we have selected vanadium as dopant element and we have supported doping by XRD and by comparison of the performance of doped samples with intimate mechanical mixtures of vanadium oxide and $\left(\mathrm{Ti}^{3+} / \mathrm{Ti}^{4+}\right) \mathrm{P}$ in similar weight proportions. As in other cases in the literature, we have found that there is an optimal percentage of doping that achieves the highest photocatalytic activity. The present chapter therefore describes a new type of photocatalyst that combines easy preparation, flexibility in 
doping, visible light photoresponse in absence of noble metals and offers many possibilities to overcome titanium dioxide as photocatalyst.

\subsection{References.}

[1] Chen, X. Mao, S. S., Chemical Reviews 2007, 107, 2891-2959.

[2] Asahi, R. Morikawa, T. Ohwaki, T. Aoki, K. Taga, Y.,Science 2001, 293, 269271.

[3] Ihara, T. Miyoshi, M. Iriyama, Y. Matsumoto, O. Sugihara, S., Applied Catalysis B-Environmental 2003, 42, 403-409.

[4] Kisch, H. Macyk, W., Chemphyschem 2002, 3, 399-404.

[5] Primo, A. Marino, T. Corma, A. Molinari, R. Garcia, H., Journal of the American Chemical Society 2011, 133, 6930-6933.

[6] Rehman, S. Ullah, R. Butt, A. M. Gohar, N. D., Journal of Hazardous Materials 2009, 170, 560-569.

[7] Maeda, K. Teramura, K. Lu, D. L. Takata, T. Saito, N. Inoue, Y. Domen, K., Nature 2006, 440, 295-295.

[8] Balzani, V. Credi, A. Venturi, M., ChemSusChem 2008, 1, 26-58.

[9] Bensaid, S. Centi, G. Garrone, E. Perathoner, S. Saracco, G., ChemSusChem 2012, 5, 500-521.

[10] Bolton, J. R., Science 1978, 202, 705-711.

[11] Centi, G. Perathoner, S., ChemSusChem 2010, 3, 195-208.

[12] Gust, D. Moore, T. A. Moore, A. L., Accounts of Chemical Research 2009, 42, 1890-1898.

[13] Lewis, N. S. Nocera, D. G., Proceedings of the National Academy of Sciences 2006, 103, 15729-15735.

[14] Serpone, N. Lawless, D. Terzian, R., Sol. Energy 1992, 49, 221-34.

[15] Gomes Silva, C. u. Juarez, R. Marino, T. Molinari, R. Garcia, H., Journal of the American Chemical Society 2011, 133, 595-602.

[16] Chen, X. Liu, L. Yu, P. Y. Mao, S. S., Science 2011, 331, 746-750.

[17] Burda, C. Lou, Y. B. Chen, X. B. Samia, A. C. S. Stout, J. Gole, J. L., Nano Letters 2003, 3, 1049-1051.

[18] Koci, K. Mateju, K. Obalova, L. Krejcikova, S. Lacny, Z. Placha, D. Capek, L.Applied Catalysis B-Environmental 2010, 96, 239-244. 
[19] Sato, S. Nakamura, R. Abe, S., Applied Catalysis a-General 2005, 284, 131137.

[20] Serpone, N., Journal of Physical Chemistry B 2006, 110, 24287-24293.

[21] Zuo, F. Bozhilov, K. Dillon, R. J. Wang, L. Smith, P. Zhao, X. Bardeen, C. Feng, P., Angew. Chem., Int. Ed. 2012, 51, 6223-6226, S6223/1-S6223/9.

[22] Murugavel, R. Choudhury, A. Walawalkar, M. G. Pothiraja, R. Rao, C. N. R., Chem. Rev. 2008, 108, 3549-3655.

[23] Ekambaram, S. Sevov, S. C., Angew. Chem., Int. Ed. 1999, 38, 372-375.

[24] Kanan, M. W. Surendranath, Y. Nocera, D. G., Chem. Soc. Rev. 2009, 38, 109114.

[25] Wang, X. J. Pang, H. Zhao, S. Shao, W. Yan, B. Li, X. Li, S. Chen, J. Du, W., ChemPhysChem 2013, 14, 2518-2524.

[26] Liu, Y. Shi, Z. Fu, Y. Chen, W. Li, B. Hua, J. Liu, W. Deng, F. Pang, W., Chem. Mater. 2002, 14, 1555-1563 
Chapter 7

Doped framework iron phosphate as novel photocatalyst for hydrogen production from water-methanol mixtures. 
Chapter 7 - Doped framework iron phosphate as novel photocatalyst for hydrogen production from water-methanol mixtures.

\subsection{Introduction.}

Photocatalysis is gaining importance because in addition to degradation of pollutants in gas o liquid phase, it could also be an alternative for solar light conversion [1-5]. Due to the shortage in fossil fuels, a considerable effort is focused on the development of renewable energies. Sunlight reaching the Earth surface is one of the primary energy resources that provides more than enough energy to cover the energy demand in the future [6-9]. However, due to the low power density of solar light, day and night cycles, as well as the dependence on weather conditions, the use of solar light as renewable energy has to be combined with some energy vector that accumulates and concentrates the solar energy. One strategy that is currently under the spotlight is the generation of hydrogen from water or the production of other solar fuels, based on solar light photocatalysis $[7$, 10-16].

In photocatalysis the energy of the light is converted by a solid into chemical energy [17-22]. Typical photocatalysts are semiconductors in which irradiation with photons of wavelength longer than the band gap leads to the promotion of one electron from the valence to the conduction band generating a state of charge separation. The most widely used photocatalyst, also for the production of hydrogen and solar fuels, is titanium dioxide [17, 19,21]. This solid presents several advantages including availability, lack of toxicity and high photocatalytic efficiency under UV light irradiation. However, $\mathrm{TiO}_{2}$ presents still severe limitations that have proven to be difficult to overcome.

As sufficiently commented in different part of this thesis one of these limitations is the lack of photocatalytic response of $\mathrm{TiO}_{2}$ under visible light irradiation due to its wide energy band gap. A second limitation is related to the occurrence of charge recombination that constitutes an energy waste pathway. For these reasons, the performance of $\mathrm{TiO}_{2}$ as photocatalyst, particularly under sunlight illumination, is far from optimal and there is a continuous interest in developing new photocatalysts. Of relevance for the present work is also the recent finding about the photoelectrochemical activity of $\alpha-\mathrm{Fe}_{2} \mathrm{O}_{3}$ powders for hydrogen generation 
from water [23-28]. One of the main advantages of iron oxide is its lack of toxicity and also that iron is the most abundant metal element in the Earth. However, iron oxide presents as mayor drawback in photocatalysis the low reduction potential of electrons in its conduction band $\left(-0.4 \mathrm{eV}{ }^{[29]}\right)$ that make thermodynamically uphill the reduction of water to form $\mathrm{H}_{2}$. To overcome this severe limitation it is necessary to submit the $\alpha-\mathrm{Fe}_{2} \mathrm{O}_{3}$-based photoanode to a bias potential, generally about $1 \mathrm{~V}$, in a photoelectrochemical cell. In addition, $\alpha-\mathrm{Fe}_{2} \mathrm{O}_{3}$ tends to undergo photocorrosion, leading to the dissolution of $\mathrm{Fe}^{3+}$ during the photocatalytic reaction. In this context it would be of interest to develop and evaluate other water-insoluble iron compounds that could perform as photocatalysts similarly to $\alpha-\mathrm{Fe}_{2} \mathrm{O}_{3}$, but without the limitations encountered in this oxide.

Considering the interest in developing new photocatalysts, in the present chapter we report the photocatalytic activity of iron phosphate (FeP) for $\mathrm{H}_{2}$ generation from $\mathrm{CH}_{3} \mathrm{OH} / \mathrm{H}_{2} \mathrm{O}$ mixture that is comparable to that of commercial P25 under simulated sunlight irradiation. Compared to $\mathrm{TiO}_{2}$, the use of $\mathrm{FeP}$ has the additional advantage of an easy doping procedure. We have found that the photocatalytic activity of FeP depends on the nature of the dopant and percentage of doping. The properties of $\mathrm{FeP}$ as semiconductor were characterized by measuring conduction the conduction band potential, band gap, density of charge carriers and by spectroscopic characterization of the charge separated state by transient absorption spectroscopy.

Since phosphates can be prepared by hydrothermal crystallization in the presence of a large variety of metals in a range of concentrations resulting in doping, the results reported here can open new directions in the search for novel efficient photocatalysts. In the literature, there is only one precedent on the use of $\mathrm{Fe}(\mathrm{OH}) \mathrm{PO}_{4}$ as photocatalyst for the degradation of methylene blue under visible light irradiation [30]. Compared with that precedent, our study focus on a different catalytic reaction with a FeP of open framework structure and we also show the possibility of metal doping to gain control on the efficiency of the photocatalytic activity of FeP. 


\subsection{Results and Discussion.}

Open framework phosphate are those materials that in contrast to bulk phosphate only contain Metal-O-P linkages leaving interstitial lattice space in where accompanying organic component, generally amines, can be accommodated. Besides FeP having a single metal element, we also performed the synthesis of a wide range of FeP having in various minor proportions $\mathrm{Cr}$, $\mathrm{Mn}$, or Co from 0.25 to 5 wt\%. Specifically, we prepared three families of materials, based on FeP, depending on nature of the dopant element. The set of materials and the main analytical and characterization data are summarized in Table 7.1.

Table 7.1. Code, composition and photocatalytic data of the FeP samples prepared in this chapter.

\begin{tabular}{|l|l|l|l|}
\hline & \multicolumn{2}{|l|}{ Code ${ }^{\text {(a) }}$} & \multicolumn{2}{|l|}{ Fe } & \\
\hline FeP & & 100 & 14.82 \\
\hline (0.25 Cr) FeP & Cr: 0.28 & 99.7 & 22.17 \\
\hline (0.5 Cr) FeP & Cr: 0.48 & 99.5 & 34.15 \\
\hline (1 Cr) FeP & Cr: 0.90 & 99.1 & 35.82 \\
\hline (2 Cr) FeP & Cr: 2.30 & 97.7 & 26.30 \\
\hline (5 Cr) FeP & Cr: 4.0 & 96.0 & 25.87 \\
\hline (0.25 Mn) FeP & Mn: 0.20 & 99.8 & 20.99 \\
\hline (0.5 Mn) FeP & Mn: 0.35 & 99.65 & 18.93 \\
\hline (1 Mn) FeP & Mn: 1.0 & 99.0 & 17.29 \\
\hline (2 Mn) FeP & Mn: 1.2 & 98.8 & 15.23 \\
\hline (5 Mn) FeP & Mn: 3.3 & 96.7 & 14.82 \\
\hline (0.25 Co) FeP & Co: 0.13 & 99.87 & 21.82 \\
\hline (0.5 Co) FeP & Co: 0.27 & 99.73 & 22.23 \\
\hline (1 Co) FeP & Co: 0.42 & 99.58 & 24.70 \\
\hline (2 Co) FeP & Co: 0.80 & 99.2 & 22.64 \\
\hline (5 Co) FeP & Co: 2.1 & 97.9 & 15.64 \\
\hline
\end{tabular}


(a) The empirical formula of FeP photocatalyst is $\left(\mathrm{C}_{2} \mathrm{~N}_{2} \mathrm{H}_{10}\right) \mathrm{Fe}_{(1-\mathrm{x})} \mathrm{M}_{\mathrm{x}}\left(\mathrm{HPO}_{4}\right)_{2}(\mathrm{OH})$. $\mathrm{H}_{2} \mathrm{O}$ with $\mathrm{M}=\mathrm{Cr}$, $\mathrm{Mn}$ or Co.

(b) $\mathrm{H}_{2}$ production after $6 \mathrm{~h}$ irradiation with solar simulator.

In the first part of the present chapter we will proceed to describe the preparation of open framework FeP that was synthetized by hydrothermal crystallization at $150{ }^{\circ} \mathrm{C}$ of an aqueous solution of iron chloride, ethylenediamine as base and templating agent, and phosphoric acid as source of phosphate. After $2.5 \mathrm{~h}$ at the required temperature, the suspension was cooled down at room temperature, filtered and washed. Those samples based on FeP exhibit a X-ray diffraction pattern that has been previously reported for the one-dimensional, open framework iron phosphate [31]. Figure 7.1. shows selected XRD patterns for some of the samples based on FeP as well as the structure for these materials as reported in the literature by Song [31, 32]. Noteworthy is the fact that the crystallinity of FeP is maintained with some minor variations in the peak intensity in the presence of the dopant elements.

Figure 7.1. Crystal structure and XRD of the samples under study. A) Building units of FeP, view along $\mathrm{b}$ axis. Red atoms correspond to iron, green to phosphor and blue to oxygen. Hydrogen atoms, ethylenediamine and water molecules are omitted for clarity. B), C) and D) XRD pattern of the metal-doped samples (a) FeP, (b) (0.25 M) FeP, (c) (0.5 M) FeP, (d) (1 M) FeP, (e) (2 M) FeP, (f) (5 M) FeP. M correspond to $\mathrm{Cr}, \mathrm{Mn}$ and Co respectively.

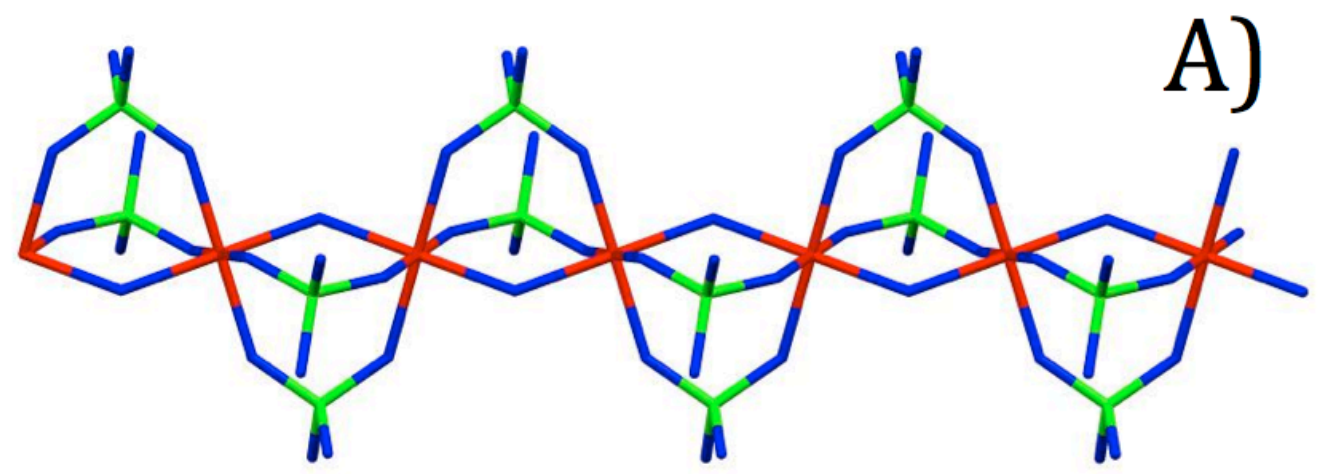



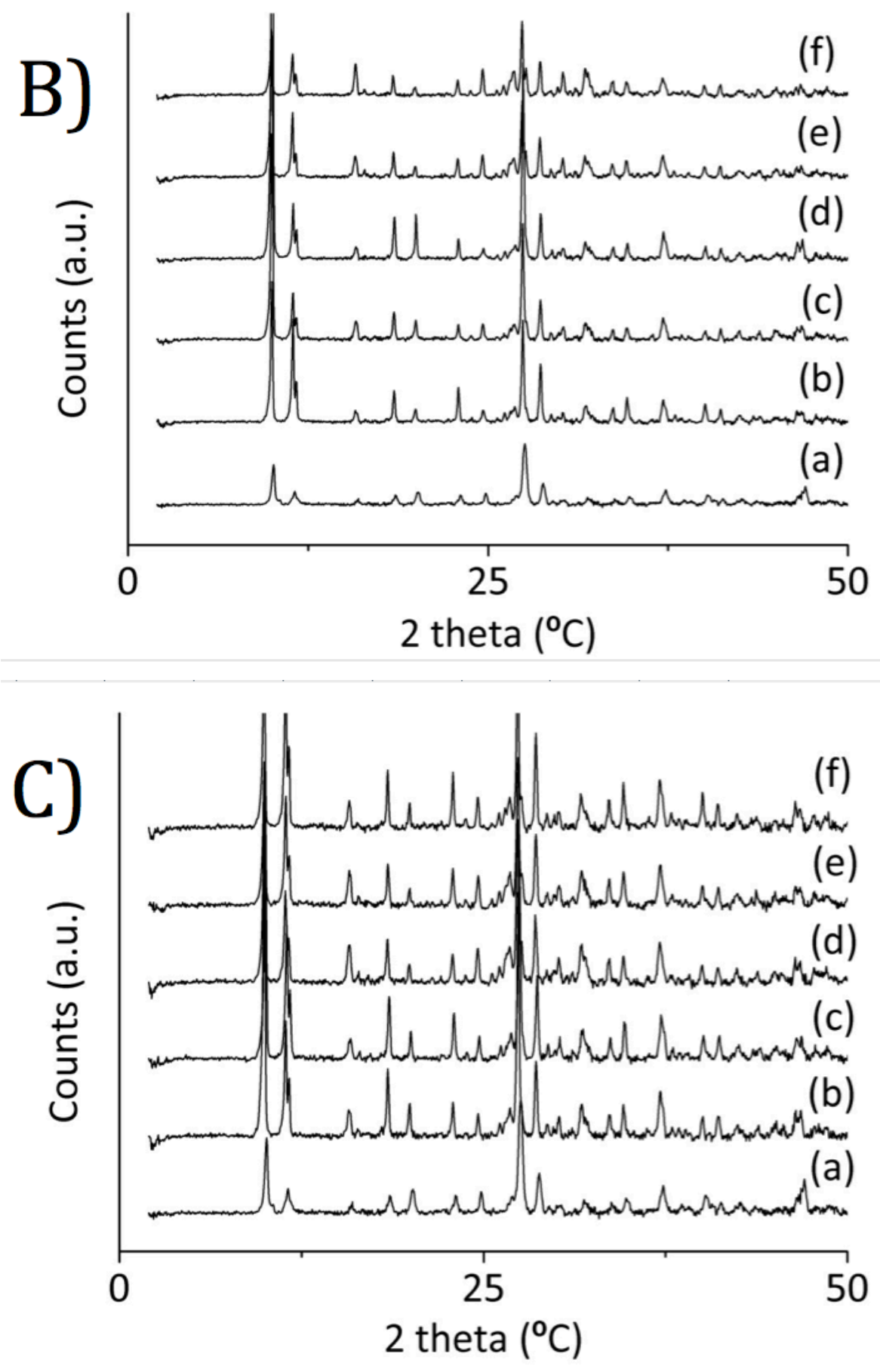


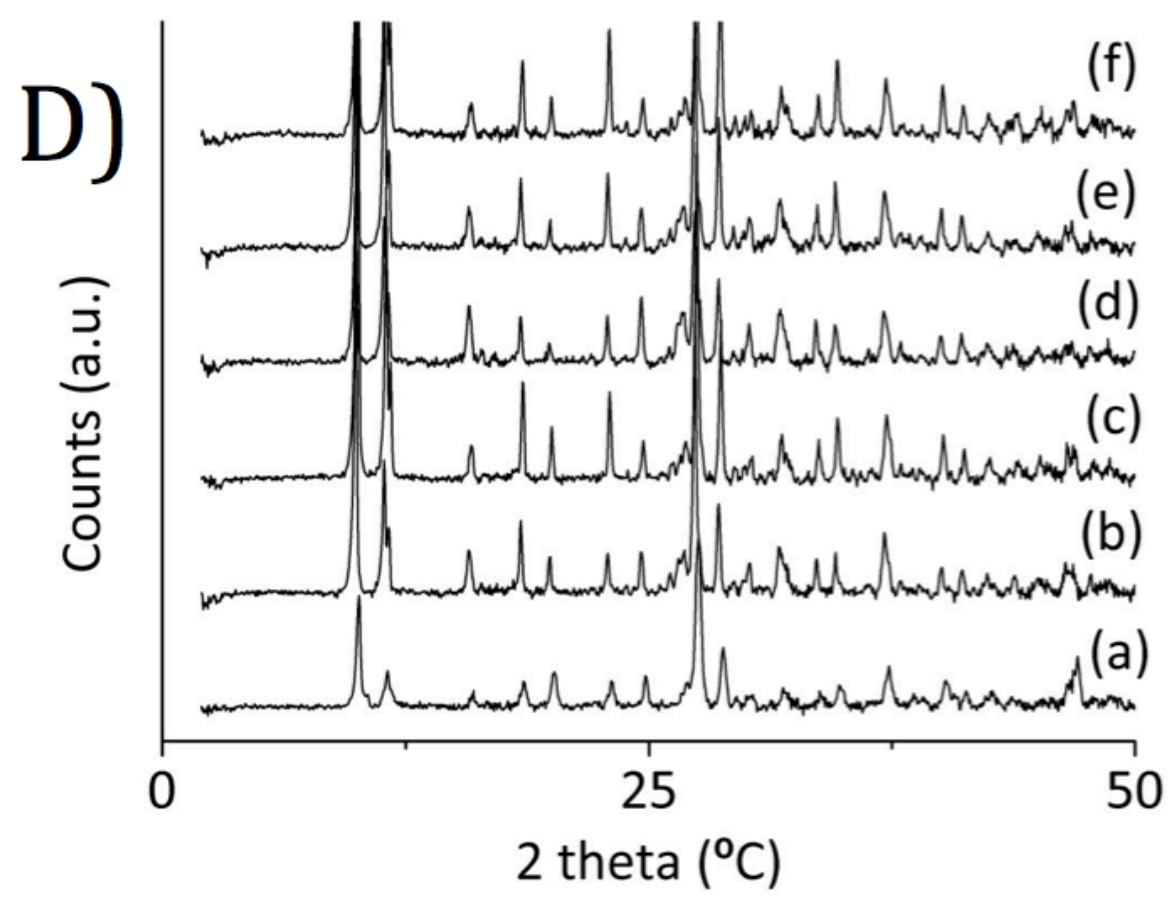

The almost coincident cationic radius of $\mathrm{Fe}$ and $\mathrm{Cr}, \mathrm{Mn}$ or $\mathrm{Co}$ as well as their similar charge density allow the incorporation of dopants elements replacing a few percent of $\mathrm{Fe}^{3+}$ ions in the structure without noticeable variation in the position of the XRD peaks. The crystal structure of FeP and related doped material is constituted by chains of octahedra having one $\mathrm{Fe}^{3+}$ ion in the centre sharing two corners in trans position with $\mathrm{OH}$ groups. Phosphates anions define tetrahedra around $\mathrm{P}^{5+}$ ions connected by two oxygen atoms to two different octahedra and having in another corner one $\mathrm{OH}$ group. Ethylendiamine is present as diprotonated form occupying the interstitial space among the chain of octahedra that are maintained by strong electrostatic forces and by hydrogen bonds.

The morphology of FeP particles was determined by microscopy. The large size of some of the particles allows in this case the use of optical microscopy. Figure 7.2. presents representative images for $\mathrm{FeP}$ showing that the material is constituted by a broad distribution of crystals with long aspect ratio. Some of the particles were as long as at $100 \mu \mathrm{m}$, but there were some other particles about $1 \mu \mathrm{m}$ length. With respect to the morphology, the material is constituted by sub-millimetric crystals that were formed by aggregation of much smaller sheets. Scanning electron microscopy reveals in better detail that the crystallites are in reality aggregates of 
multitude of flakes with thickness about $0.5 \mu \mathrm{m}$ and length typically larger than 20 $\mu \mathrm{m}$ (see also Figure 7.2B).

Figure 7.2. A) Optical microscopy image at different magnification showing the large crystals present in FeP. B) SEM image recorded for FeP and EDX of the particle showing it elemental composition

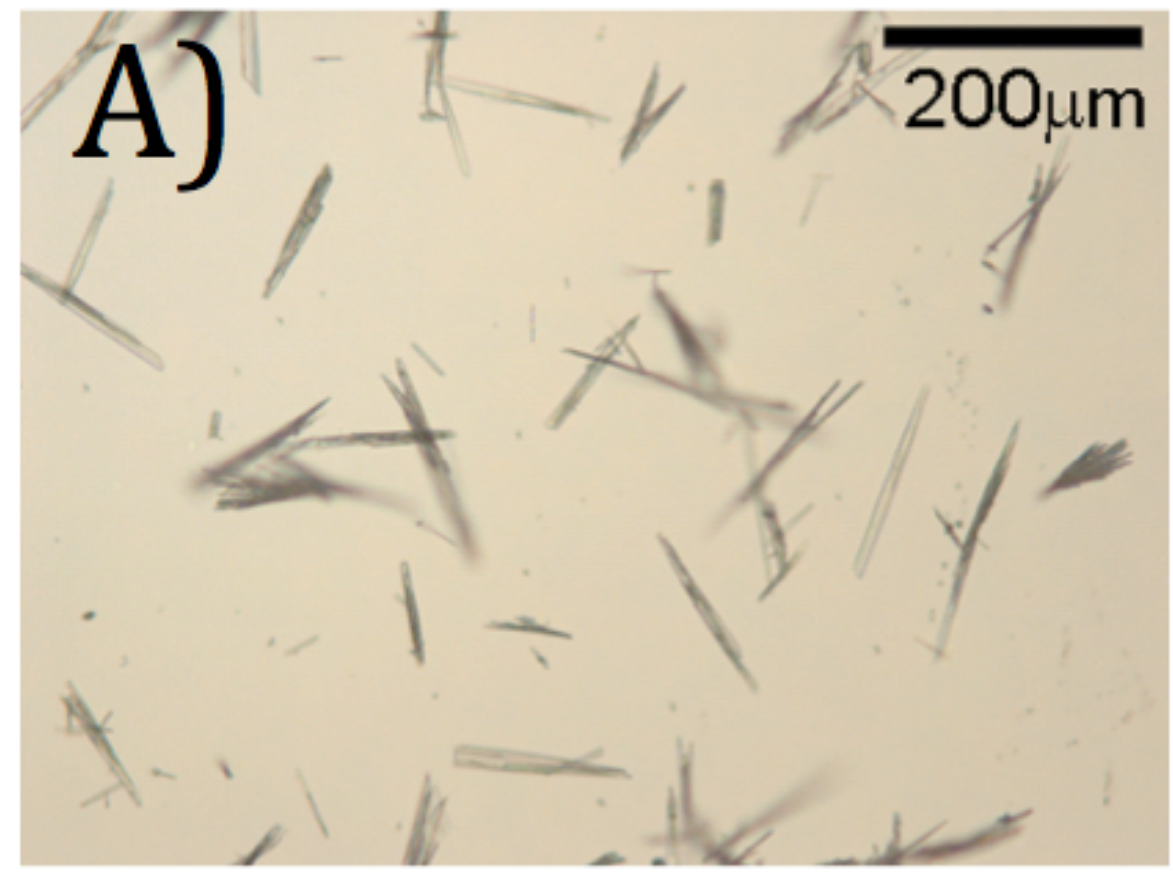



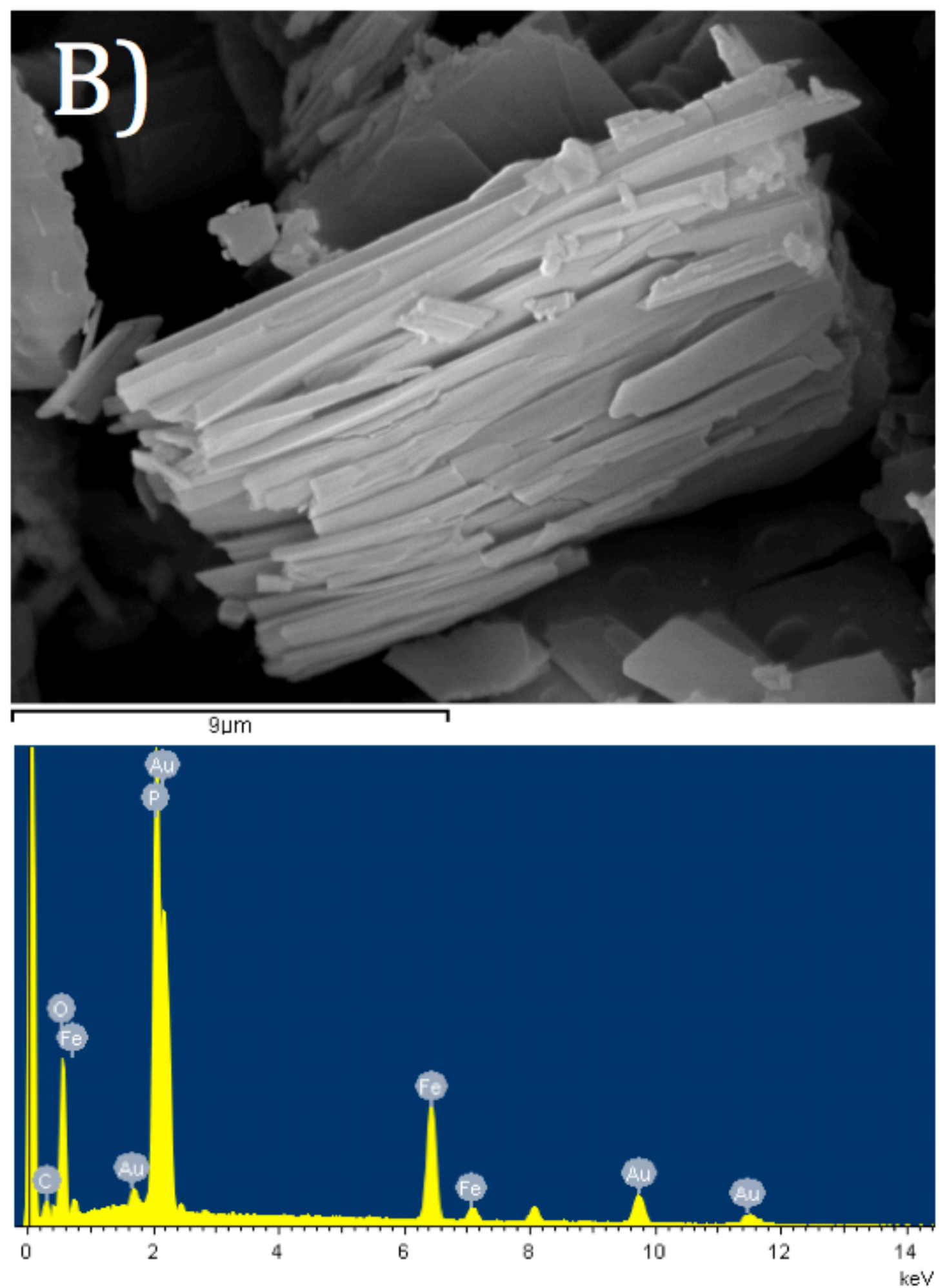
Of relevance with regard to the photocatalytic activity is the optical absorption spectra of the FeP samples that were coincident regardless the nature of the dopant element and its percentage in the range from 0.25 to $5 \mathrm{wt} \%$, as shown in Figure 7.3. All the samples exhibited two relative absorption maxima at 380 and $420 \mathrm{~nm}$ that can be attributed to electronic d-d transitions of $\mathrm{Fe}^{3+}$ ions. These minor peaks are accompanied by an intense absorption band at $280 \mathrm{~nm}$ attributable to the ligand-to-metal O-Fe charge transfer band.

All the FeP samples under study exhibited photocatalytic activity for $\mathrm{H}_{2}$ generation under simulated solar light irradiation in $\mathrm{H}_{2} \mathrm{O} / \mathrm{CH}_{3} \mathrm{OH}$ mixture. Figure 7.4. shows the temporal profile of hydrogen evolution for the undoped FeP sample.

Figure 7.3. Diffuse reflectance UV-Vis absorption spectra (plotted as the KubelkaMunk function of the remittance (R)) of the metal-doped FeP.

A), B) and C) (a) FeP, (b) (0.25 M) FeP, (c) (0.5 M) FeP, (d) (1 M) FeP, (e) (2 M) FeP, (f) (5 M) FeP. M . M correspond to Cr, Mn and Co respectively.

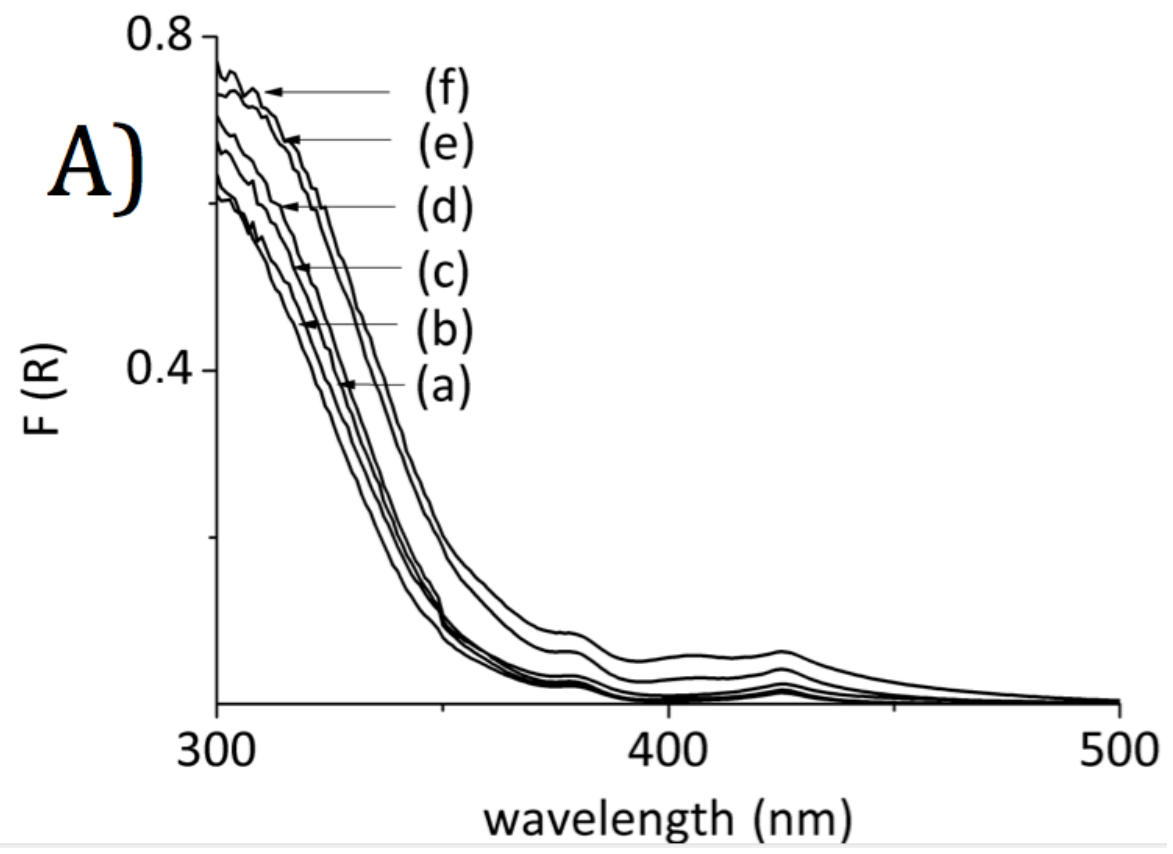



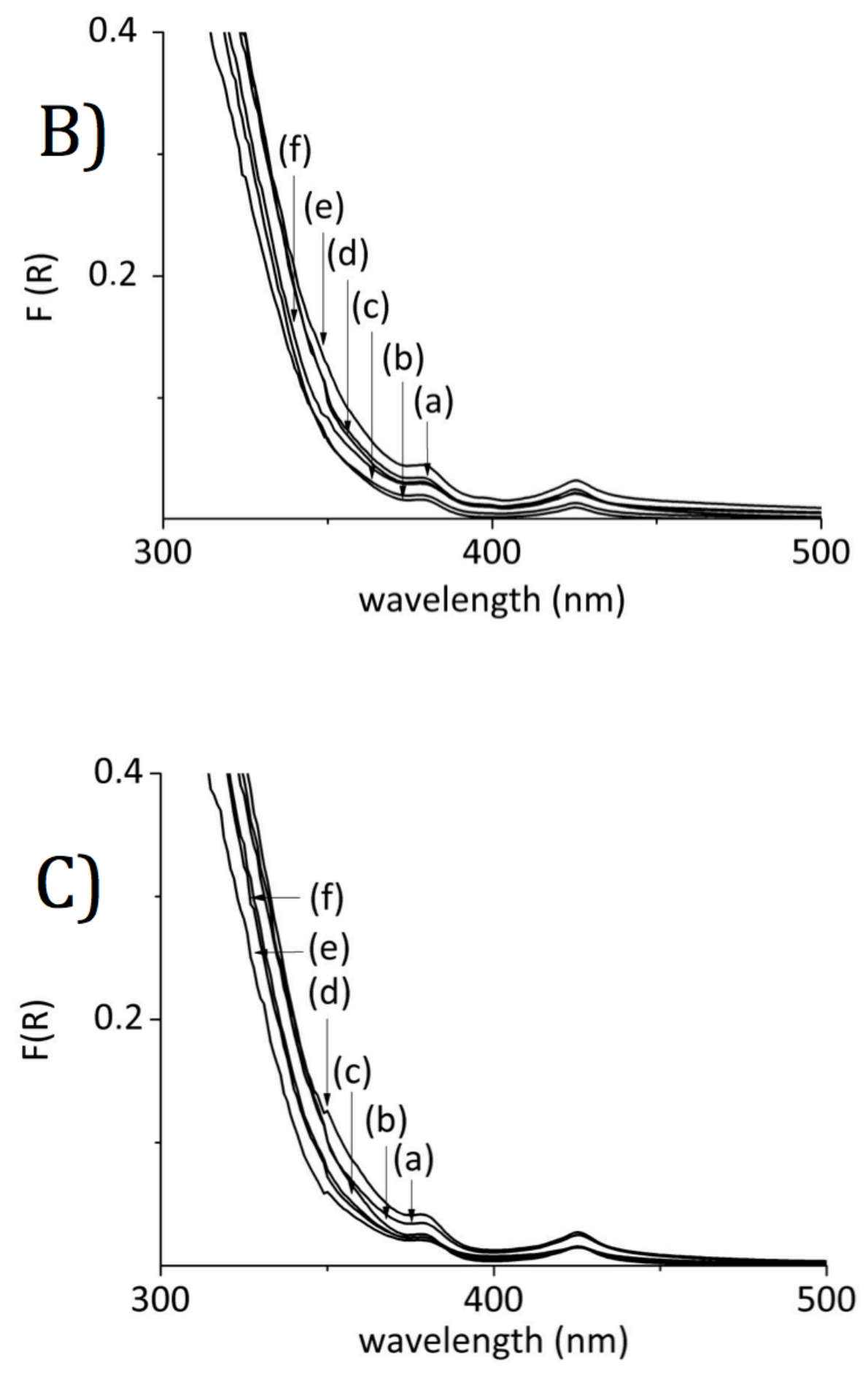

The sample exhibits an induction period of about $1 \mathrm{~h}$ in which $\mathrm{H}_{2}$ does not evolve. This induction period could be related to possible modifications in the oxidation state of $\mathrm{Fe}^{3+}$ in $\mathrm{FeP}$ or to the adsorption of hydrogen onto the solid. It is important to mention that this $\mathrm{H}_{2}$ production corresponds to a photocatalyst that does not contain any noble metal, such as Pt, as cocatalyst. 
Figure 7.4. Photocatalytic hydrogen production from water-methanol mixtures using $\mathrm{FeP}$ as photocatalyst under simulated sunlight irradiation. Reaction conditions: Water-methanol (1:1) mixtures $25 \mathrm{~mL}$, catalyst concentration $1 \mathrm{~g} \times \mathrm{L}^{-1}$.

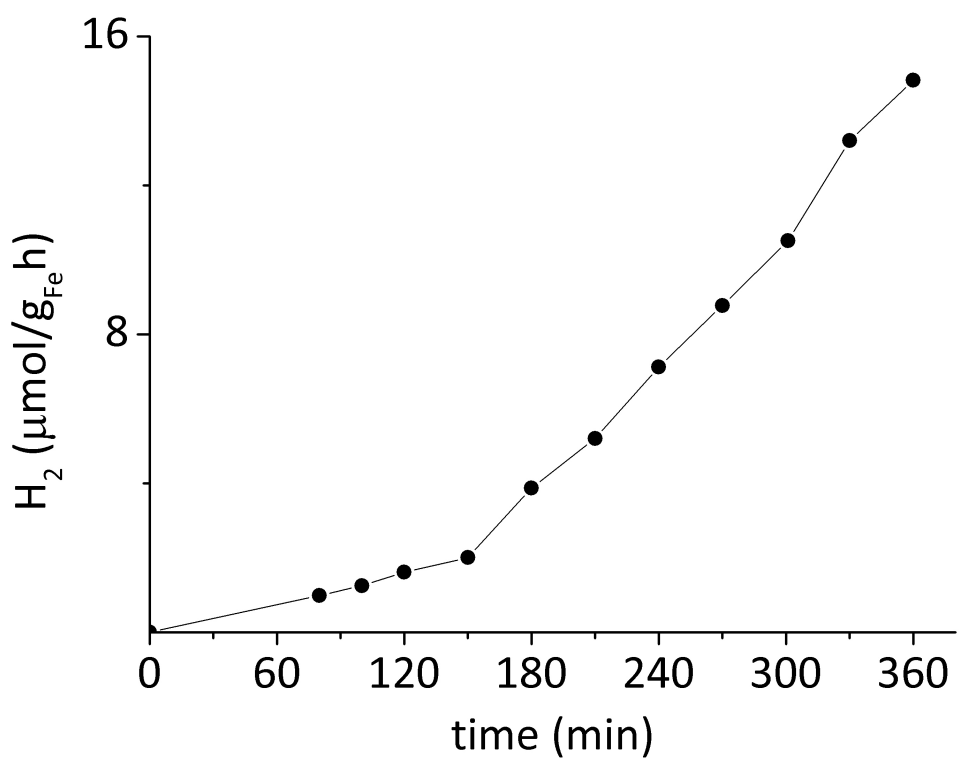

Concerning stability, chemical analysis of the solution after $6 \mathrm{~h}$ irradiation and removal of the solid catalyst shows an iron content of $0.040 \mathrm{ppm}$, corresponding to $0.026 \%$ of the initial $\mathrm{Fe}^{3+}$ present in the photocatalyst, thus, indicating that $\mathrm{Fe}$ leaching is negligible under the present conditions.

This analytical data showing negligible $\mathrm{Fe}^{3+}$ leaching together with the lack of apparent change in the XRD pattern of the FeP samples after prolonged irradiation indicate the stability of FeP as photocatalyst. The results shown in Figure 7.4. have to be considered in the context of the lack of hydrogen production in the absence of bias potential of $\alpha-\mathrm{Fe}_{2} \mathrm{O}_{3}{ }^{[26]}$ and the low photostability of this oxide resulting in leaching $\mathrm{Fe}^{3+}$ to the solution and dissolution of the solid [28].

The influence of doping on the photocatalytic activity for hydrogen generation is presented in Figure 7.5. As can be seen there for the three sets of samples, the activity for hydrogen production is optimal when the dopant of loading is between 0.1 and $1 \mathrm{wt} \%$, lower or higher loadings resulting in a decrease in the photocatalytic activity. Also the nature of the dopant element has an influence, chromium being the dopant metal for which the photocatalytic activity is the highest. According to the data shown in Figure 7.5. the amount of hydrogen can be 
increased by a factor 2.5 by incorporating in the composition chromium in a percentage about $1 \%$.

Figure 7.5. Hydrogen production from water-methanol mixtures at $6 \mathrm{~h}$ irradiation using doped FeP as photocatalysts under simulated sunlight irradiation. Reaction condition: water-methanol mixtures (1:1) $25 \mathrm{~mL}$, catalyst concentration $1 \mathrm{~g} \times \mathrm{L}^{-1}$. From left to right concentration of dopant element grows from: $0,0.25,0.5,1,2$ and $5 \%$.

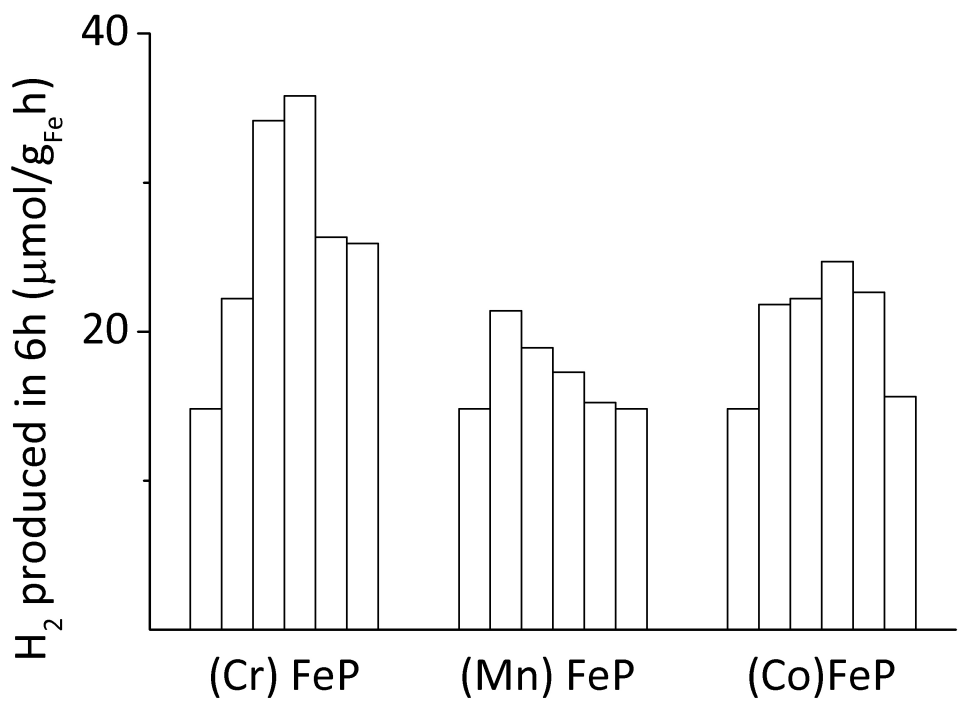

The above data refer to irradiations with simulated solar light that contains about $4 \%$ of the energy in the UV region and about $42 \%$ of the energy in the visible zone. Due to the lack of photocatalytic activity of titanium oxide under irradiation with visible light, there is a continuous interest in developing visible light photocatalysts.

In the present case, and considering that according to the absorption spectra the samples absorb light in the visible region, we were interested in determining if the doped FeP samples exhibit some photocatalytic activity with visible light. Aimed at addressing this issue we performed a set of photocatalytic tests using a LED emitting light of wavelength above $450 \mathrm{~nm}$ (see emission spectra in the Chapter 8.2.). All the samples exhibit under this condition weak photocatalytic activity. 
Figure 7.6. summarizes the hydrogen generation of Cr-doped FeP samples under visible light. The results obtained indicate that under simulated sunlight a considerable proportion, about $66 \%$, of the photocatalytic activity is due to the UV light, despite that this wavelength zone corresponds to a minor percentage to the total solar energy.

Figure 7.6. Hydrogen production from water-methanol mixtures using ( $\mathrm{Cr}$ ) FeP as photocatalysts and visible-light LED as irradiation source. Reaction conditions: Water-methanol mixture (1:1) $25 \mathrm{~mL}$, catalyst concentration $1 \mathrm{~g} \times \mathrm{L}^{-1}$, time $24 \mathrm{~h}$. From left to right concentration of dopant element grows from: $0,0.25,0.5,1,2$ and $5 \mathrm{wt} \%$.

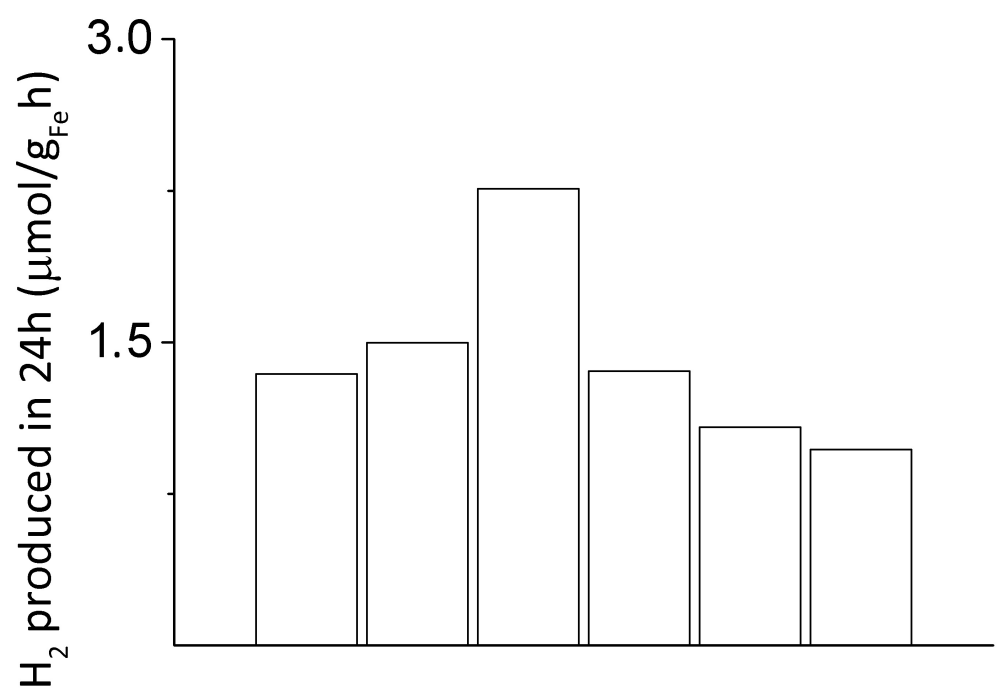

(Cr) FeP

In analogy with $\mathrm{TiO}_{2}$, we propose that in the present case metal doping should operate by increasing the efficiency of charge separation. It is known that upon light absorption and generation of electrons in the conduction band and holes in the valence band, prompt charge recombination is the main deactivation pathway, limiting the efficiency of the photocatalytic reactions [17].

Doping can introduce in the intra bandgap region additional states that can act as traps of electrons or holes, promoting charge separation and/or increasing the lifetime of the charge separated estate. Scheme 7.1. summarizes our proposal to 
explain the influence of doping and the more efficient hydrogen generation of doped semiconductors. The fact that there is an optimal doping level suggests that the positive effect of the dopant increasing the efficiency of charge separation by providing intra bandgap trapping sites is compensated by an increase in charge recombination efficiency caused at higher dopant concentrations [21].

Scheme 7.1. Proposal to rationalize for the influence of metal doping on the charge separation and hydrogen production of doped FeP. VB: valence band CB: conduction band. The dash line below CB correspond to the interband gap state introduced by the dopant element.

(a) Charge separation; (b) Migration of conduction band electrons to the dopant level; (c) $\mathrm{H}^{+}$reduction.

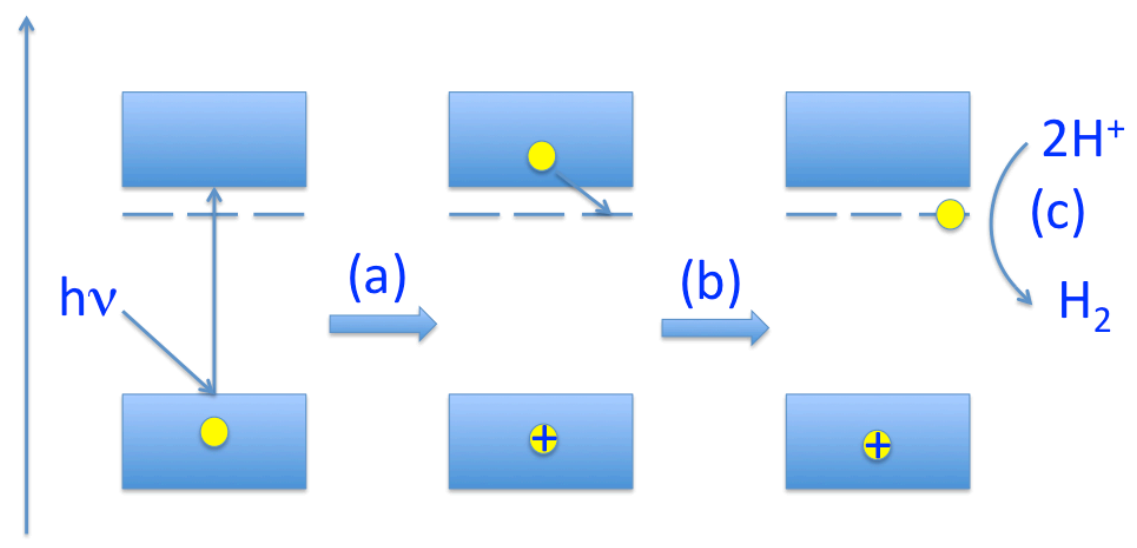

Estimation of the conduction flat band potential for FeP was carried out measuring the photocurrent against the bias potential, determining the value that change the photocurrent from positive to negative. These measurements give a flat band potential of $0.1 \mathrm{eV}$. Alternately, impedance spectroscopy of thin FeP films on transparent conductive electrode allowed also to determine the flat band potential and the density of charge carriers that was estimated based on this technique as $0.1 \mathrm{eV}$ and $2.6 \cdot 10^{20}$ carriers $/ \mathrm{cm}^{3}$.

The charge separation state, and its quenching behaviour as well as the influence of the metal doping were monitored by transient absorption spectroscopy using $266 \mathrm{~nm}$ laser as excitation wavelength. In these experiments FeP samples were suspended in acetonitrile by sonication. After removal of the solid residues by 
decantation, the persistent FeP colloidal solutions were submitted to laser flash excitation. Regardless the presence of dopant elements all the transient spectra were characterized by a continuous absorption spanning the whole wavelength range. As an example, Figure 7.7. shows the transient spectra for FeP reported at 1.9, 5.8 and $10.8 \mu$ s after the laser flash. The temporal profile of the signal monitored at different wavelength was coincident, suggesting that they corresponds to the same transient. The temporal profile has three regimes (see Figure 7.7.).

Figure 7.7. Transient Spectra recorded for FeP $\mathrm{N}_{2}$-purged $\mathrm{FeP}$ suspended in acetonitrile (O.D. at $355 \mathrm{~nm} \mathrm{0.3)} \mathrm{at} 0.26(\mathbf{\square}), 5.8(\mathbf{O})$ and $10.78(\mathbf{\Delta}) \mu s$ after 355 nm laser excitation. Inset A: temporal profile of the transient spectrum. The profile shows three kinetic regimes that have been labelled as $A, B$ and $C$, respectively. Inset B: temporal profile of the signal recorded for at $420 \mathrm{~nm}$ for suspension in acetonitrile (O.D. at $355 \mathrm{~nm} \mathrm{0.31)}$ after $355 \mathrm{~nm}$ laser excitation. The plots $\mathrm{a}, \mathrm{b}$ and c corresponds to the temporal profiles after $\mathrm{N}_{2}$ and $\mathrm{O}_{2}$ purging or in presence of methanol, respectively.

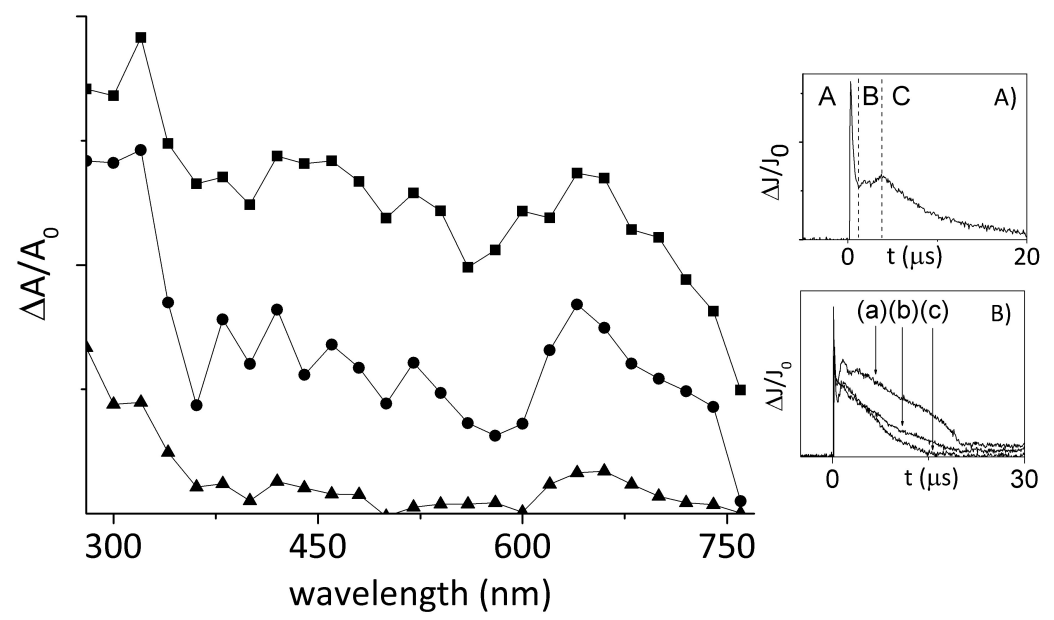

The first one is a fast decay, taking place in less than $0.15 \mu \mathrm{s}$ and corresponding to more than $60 \%$ of the top $\Delta \mathrm{J} / \mathrm{J}_{\mathrm{o}}$ (noted with the letter $\mathrm{A}$ in inset $\mathrm{A}$ ) of Figure 7.7.). This fast decay is followed by a growth from 0.15 to about $2.0 \mu$ s that increases the $\Delta \mathrm{J} / \mathrm{J}_{\mathrm{o}}$ signal about $10 \%$ (noted with the letter B in inset A) of Figure 7.7.). The last 
kinetics correspond to the final decay of the signal that take place from 2.5 to 20 us. As we will see below, quenching experiments support that this transient correspond to the charge separated state of FeP materials. Then, according this, the three kinetics could correspond to fast charge recombination (regime A), followed by charge migration and relocation into traps (regime B) and final recombination of free charge carriers (regime $\mathrm{C}$ ).

No influence of the dopant elements either on the spectra or on temporal profiles was observed. However, we notice that the intensity of the signal immediately after the laser pulse was dependent on the nature of dopant. This observation could suggest that the influence of the dopant element is more on the generation of charge carriers than in the location or recombination kinetics since the latter will lead to changes in the transient spectra or in the transient signals.

Temporal profile of the signal was sensitive to the presence of quenchers (Figure 7.7.). In the present study we use oxygen as conduction band electron quencher and methanol as valence band hole quencher. In the case of FeP, the presence of oxygen increases the intensity of the signal corresponding to regimes $\mathrm{A}$ and $\mathrm{C}$ (Figure 7.7.). Methanol increases more significantly the signal in region C (Figure 7.7.). This effect of the quencher increasing the intensity of the signal without affecting much to the kinetics of the decay can be interpreted considering that geminate charge recombination of electron and holes is the main decay pathway of the signal. If one quencher is present removing partially one of the charge carriers, then, the transient signal can increase due to the detection of the complementary charge that has not collapsed by recombination and, therefore, reaches the nanosecond time scale in larger concentrations. The influence of oxygen and methanol in the temporal profile of the signal is in agreement with assignment of the transient spectra to the combined absorption of electrons and holes. The transient signal detected in the microsecond timescale being a fraction of the total charge separation events, specifically, the population of charge separated states that disappear in the sub microsecond time scale. As an example, Figure 7.7. panel B) shows the temporal profile of the signal monitored for FeP under argon or oxygen purging flow or in presence of methanol. Interestingly, doped FeP follows the same general trend observed for undoped FeP. The most salient distinctive feature of $\mathrm{O}_{2} / \mathrm{CH}_{3} \mathrm{OH}$ quenching for doped $\mathrm{FeP}$ was that the influence of quenchers, 
particularly in the regime $\mathrm{C}$ is considerably smaller than that presented in Figure 7.7. for FeP as can be seen in Figure 7.7. Regardless the top $\Delta \mathrm{J} / \mathrm{J}_{0}$, at short delay times the signal for doped FeP was clearly influenced by the presence of the quenchers, but the signal decay at larger times was almost insensitive to the presence of $\mathrm{O}_{2}$ or $\mathrm{CH}_{3} \mathrm{OH}$. This could be due to the trapping charge carriers in certain sites.

\subsection{Conclusions.}

Overall the present results illustrate the potential of widely available $\mathrm{FeP}$ as photocatalyst for hydrogen production, combining advantages of photostability, higher reduction potential of conduction band electrons than $\alpha-\mathrm{Fe}_{2} \mathrm{O}_{3}$ and easy doping during the synthesis of the materials. Our results show that doping with metal elements can increase the photocatalytic activity of the FeP by a factor of 2.5. The behaviour of FeP as semiconductor has been supported by photocurrent measurements and impedance spectroscopy that have allowed to establish the position of the conduction and valence band and an estimation of the density of carriers. The state of charge separation has been detected by transient absorption spectroscopy. It was found that the spectrum consist of a continuous absorption band spanning the whole wavelength range exhibiting three kinetic regimes and decaying completely in $20 \mu \mathrm{s}$. Assignment of the transient spectrum was based on the behaviour of the signal in the presence of oxygen and methanol that are typical electron and hole quencher, respectively.

Dopant elements influence the intensity of the transient signal that is more intense due to the dopant and also the interaction with quenchers that makes the materials less sensitive to quenching.

Thus, the present findings can open the way for further development of a novel family of semiconductors with promising applications as photocatalysts that are based on affordable elements iron and phosphor. 


\subsection{References.}

[1] Y. Amao, Chemcatchem 2011, 3, 458.

[2] G. Centi, S. Perathoner, ChemSusChem 2010, 3, 195.

[3] D. Gust, T. A. Moore, A. L. Moore, Accounts of Chemical Research 2009, 42, 1890.

[4] L. HammarstroÌ`m, S. Hammes-Schiffer, Accounts of Chemical Research 2009, 42, 1859.

[5] N. Serpone, D. Lawless, R. Terzian, Solar Energy 1992, 49, 221.

[6] D. Abbott, Proceedings of the Ieee 2010, 98, 42.

[7] S. Dunn, International Journal of Hydrogen Energy 2002, 27, 235.

[8] P. V. Kamat, Journal of Physical Chemistry C 2007, 111, 2834.

[9] N. S. Lewis, D. G. Nocera, Proceedings of the National Academy of Sciences 2006, 103, 15729.

[10] A. J. Bard, M. A. Fox, Accounts of Chemical Research 1995, 28, 141.

[11] S. Bensaid, G. Centi, E. Garrone, S. Perathoner, G. Saracco, ChemSusChem 2012, 5, 500 .

[12] X. Chen, S. Shen, L. Guo, S. S. Mao, Chemical Reviews 2010, 110, 6503.

[13] G. W. Crabtree, M. S. Dresselhaus, M. V. Buchanan, Physics Today 2004, 57, 39.

[14] M. Gratzel, Accounts of Chemical Research 1981, 14, 376.

[15] M. Ni, M. K. H. Leung, D. Y. C. Leung, K. Sumathy, Renewable and Sustainable Energy Reviews 2007, 11, 401.

[16] J. Nowotny, C. C. Sorrell, L. R. Sheppard, T. Bak, International Journal of Hydrogen Energy 2005, 30, 521.

[17] D. W. Bahnemann, Research on Chemical Intermediates 2000, 26, 207.

[18] M. A. Fox, M. T. Dulay, Chemical Reviews 1993, 93, 341.

[19] A. Fujishima, X. Zhang, D. A. Tryk, Surf. Sci. Rep. 2008, 63, 515.

[20] J. M. Herrmann, Catalysis Today 1999, 53, 115.

[21] A. L. Linsebigler, G. Q. Lu, J. T. Yates, Chemical Reviews 1995, 95, 735.

[22] A. Mills, S. LeHunte, Journal of Photochemistry and Photobiology a-Chemistry $1997,108,1$. 
[23] N. Beermann, L. Vayssieres, S. E. Lindquist, A. Hagfeldt, Journal of the Electrochemical Society 2000, 147, 2456.

[24] U. Bjorksten, J. Moser, M. Gratzel, Chemistry of Materials 1994, 6, 858.

[25] Y.-S. Hu, A. Kleiman-Shwarsctein, A. J. Forman, D. Hazen, J.-N. Park, E. W. McFarland, Chemistry of Materials 2008, 20, 3803.

[26] A. Kay, I. Cesar, M. Graetzel, Journal of the American Chemical Society 2006, 128,15714 .

[27] K. Sivula, F. Le Formal, M. Graetzel, ChemSusChem 2011, 4, 432.

[28] K. Sivula, R. Zboril, F. Le Formal, R. Robert, A. Weidenkaff, J. Tucek, J. Frydrych, M. Graetzel, Journal of the American Chemical Society 2010, 132, 7436.

[29] M. Gratzel, Nature (London, U. K.) 2001, 414, 338.

[30] X. J. Wang, H. Pang, S. Zhao, W. Shao, B. Yan, X. Li, S. Li, J. Chen, W. Du, ChemPhysChem 2013, 14, 2518.

[31] Y. Song, P. Y. Zavalij, N. A. Chernova, M. Suzuki, M. S. Whittingham, Journal of Solid State Chemistry 2003, 175, 63.

[32] Y. Song, P. Y. Zavalij, N. A. Chernova, M. S. Whittingham, Chemistry of Materials 2005, 17, 1139. 
Chapter 8 Materials and Method. 
Chapter 8 - Materials and Method.

\subsection{Emission spectra of solar simulator}

Figure 8.1. Emission Spectra of solar simulator employed as irradiation source

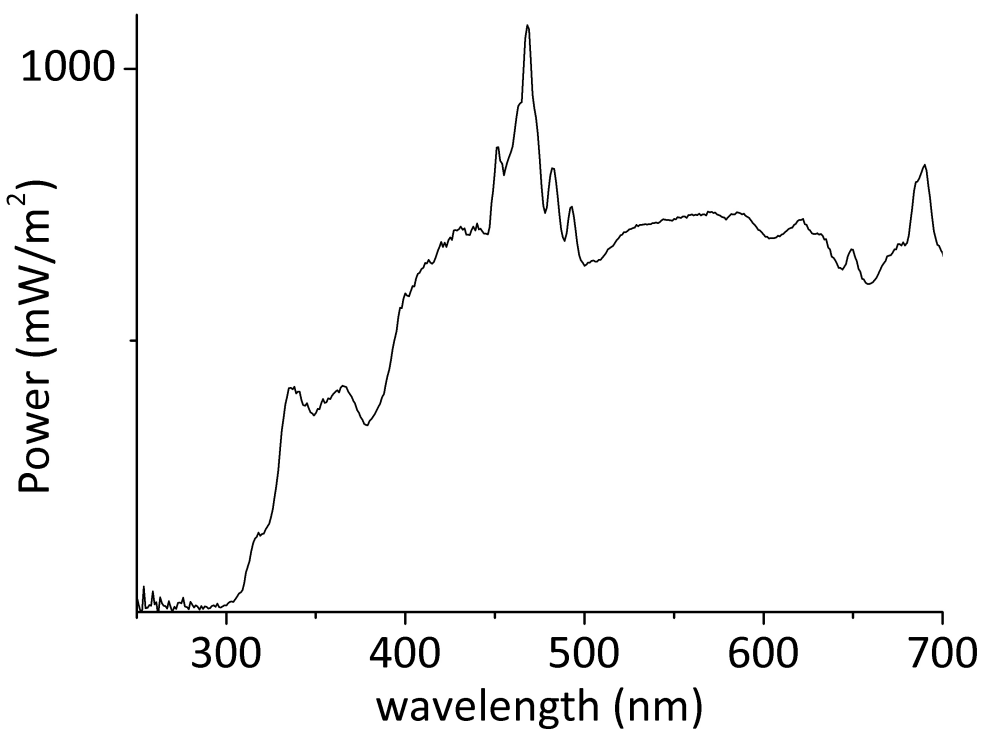

\subsection{Emission spectra visible light LED}

Figure 8.2. Emission Spectra of visible-light LED employed as irradiation source.

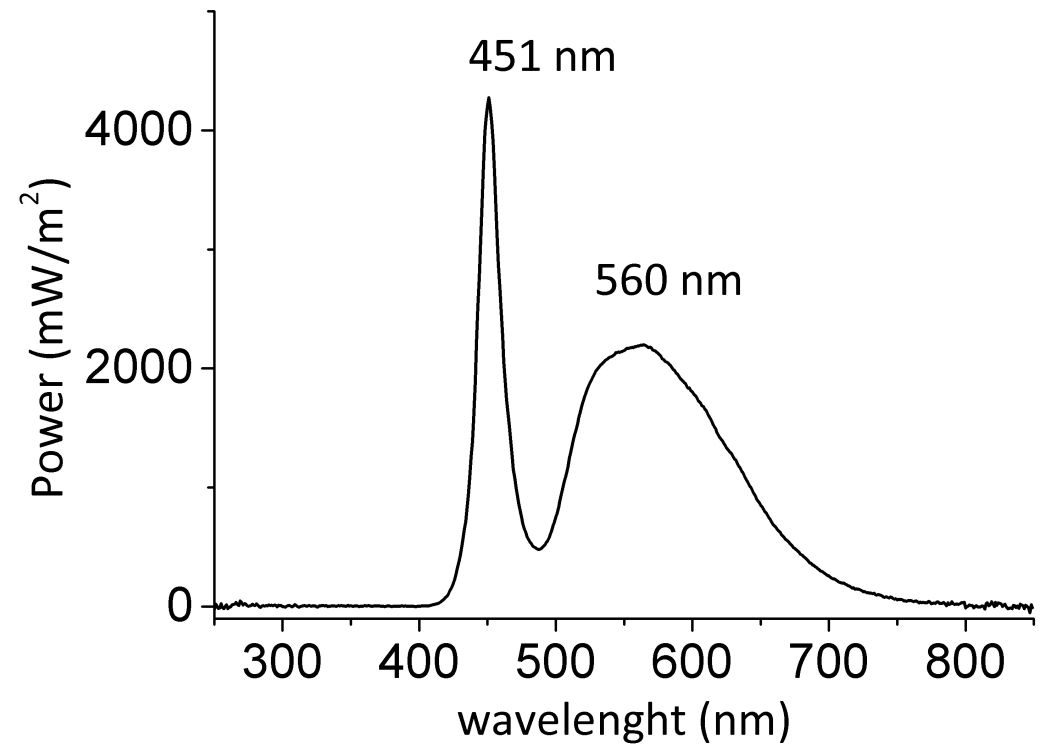




\subsection{Influence of the irradiation wavelength on the photocatalytic activity of Au-Pt nanoalloys supported on $\mathrm{TiO}_{2}$ for hydrogen generation from water.}

\subsubsection{Samples preparation.}

Pre-treated commercial titania P25 (calcination at $450{ }^{\circ} \mathrm{C}$ for $3 \mathrm{~h}$ ) was added to 100 $\mathrm{mL}$ basic aqueous solutions ( $\mathrm{pH} 10$ adjusted with $0.2 \mathrm{M} \mathrm{NaOH}$ ) containing 0.25 wt $\%$ of $\mathrm{HAuCl} 4 \cdot 3 \mathrm{H}_{2} \mathrm{O}$ and $\mathrm{H}_{2} \mathrm{PtCl}_{6} \cdot 6 \mathrm{H}_{2} \mathrm{O}$ in different molar proportions. The resulting suspensions were stirred for $20 \mathrm{~h}$ and the solid was filtered, washed with MilliQ water ( $1 \mathrm{~L}$ per gram of catalyst) and dried overnight at room temperature under vacuum. Au-Pt supported nanoalloys were finally obtained by sample reduction using a $\mathrm{H}_{2}$ flow $\left(80 \mathrm{~mL} \mathrm{~min}^{-1}\right)$ in an oven at $300 \mathrm{~K}$ for $5 \mathrm{~h}$.

\subsubsection{Sample characterization.}

X-ray photoelectron spectroscopy (XPS) measurements were performed on a SPECS spectrometer with a Phoibos MCD-9 detector and using a nonmonochromatic Al Ka (1486.6 eV) X-ray source. Spectra were recorded using an analyzer pass energy of $50 \mathrm{~V}$, an X-ray power of 50 Wand under an operating pressure of $10^{-9}$ mbar.

Spectrum treatment has been performed using the CASA software. Binding energies were referenced to $\mathrm{C} 1 \mathrm{~s}$ at $284.5 \mathrm{eV}$.

\subsubsection{Photocatalytic tests for hydrogen production.}

Visible light hydrogen generation using monochromatic $532 \mathrm{~nm}$ excitation was performed with the second harmonic of a Nd-YAG laser working with a laser power fixed to about $300 \mathrm{~mW}$ per pulse and the time width was $7 \mathrm{~ns}$ at fwhp. Aqueous or methanol : water (20:80 wt\%) suspensions of the different photocatalysts $(2 \mathrm{~mL}$ total volume with $0.8 \mathrm{wt} \%$ of the solid under optimized conditions) were placed in a capped quartz cuvette and purged with Ar for $10 \mathrm{~min}$. The hydrogen produced by each photocatalyst after irradiating with different number of laser pulses was determined by injecting $50 \mu \mathrm{L}$ of the head into a GC coupled with a TCD detector using $\mathrm{N}_{2}$ as calibration standard. Photocatalytic tests for hydrogen production using monochromatic $300 \mathrm{~nm}$ irradiation from a Hamamatsu LC8 source were carried out placing purged (2 psi of Ar for 15 min) 
and sonicated (15 min) suspension of the catalyst (25 mL, $0.5 \mathrm{~g} \mathrm{~L}^{-1}$ ) in a closed reactor with an irradiation window of $12.56 \mathrm{~cm}^{2}$ provided with temperature and pressure controllers. The evolution of hydrogen during time of irradiation was measured.

Photoreaction using a solar simulator was performed using a Thermo Oriel 1000W with an irradiation spot of $100 \mathrm{~cm}^{2}$. The light of the solar simulator was filtered through an Air Mass 1.5 filter, which contains approximately 5\% of UV radiation. Hydrogen generation was analyzed vs. time of irradiation. The amount of hydrogen generated in all the experiments was determined by sampling $50 \mu \mathrm{L}$ of the gas contained in the headspace of the sample containers with a gas syringe and injected into a calibrated gas chromatograph using nitrogen for quantification.

\subsection{Photocatalytic hydrogen generation from water-methanol mixtures using} “black" anatase obtained by annealing of titanate nanotubes.

\subsubsection{Sample preparation.}

Five titanium samples (M0-M4) were used in the present study the $\mathrm{TiO}_{2}$ precursor (M0) used for nanotube production was a commercial $\mathrm{TiO}_{2}$ powder (Aldrich, Anatase) consisting of pure anatase phase. $3.6 \mathrm{~g}$ of $\mathrm{TiO}_{2}$ powder (precursor $\mathrm{M} 0$ ) was treated with $90 \mathrm{~mL}$ of aqueous $10 \mathrm{M} \mathrm{NaOH}$ solution in a Teflon-lined autoclave (total volume $200 \mathrm{~mL}$ ) at $125{ }^{\circ} \mathrm{C}$ for 4 days. After treatment the solid was collected by filtration and washed by suspending the solid with $2 \mathrm{~L} \mathrm{HCl}$ solution $(\mathrm{pH}=1.5)$ and finally washed with water until neutral pH. Sample M1 was obtained after drying at $120^{\circ} \mathrm{C}$ for 2 days. The final annealing process give rise to two samples denoted as M2 and M3 that were obtained placing $150 \mathrm{mg}$ of M1 in a tubular quartz reactor using a $\mathrm{H}_{2}$ flow of $100 \mathrm{ml} / \mathrm{min}$ during $5 \mathrm{~h}$ at $350^{\circ}$ (sample M2) or 500 (sample M3) ${ }^{\circ} \mathrm{C}$. An additional sample denoted as M4 was obtained by heating the commercial sample $\mathrm{M} 0$ in a quartz reactor at 500C under a $\mathrm{H}_{2}$ flow (100 $\mathrm{ml} / \mathrm{min}$ ) for $1 \mathrm{~h}$ time as it was reported in the literature. [1]

\subsubsection{Photocatalytic tests for hydrogen production.}

A suspension of the catalyst $\left(25 \mathrm{~mL}, 1 \mathrm{~g} \times \mathrm{L}^{-1}\right)$ was sonicated for $10 \mathrm{~min}$ and placed in a closed reactor with an irradiation window of $12.56 \mathrm{~cm}^{2}$ provided with 
temperature and pressure controllers. The reactor was placed in a thermostatic bath with a set point temperature of $25^{\circ} \mathrm{C}$. The suspension was purged with an argon flow of 2 psi for 15 min prior to irradiation. The photoreactions were performed by using the third $(355 \mathrm{~nm})$ or the second (532 $\mathrm{nm}$ ) harmonic of a Nd:YAG laser (Lumonics, $50 \mathrm{~mJ} \times$ pulse $^{-1}, 1 \mathrm{~Hz}$ ) and a solar simulator (Thermo Oriel $1000 \mathrm{~W}$ ) with an irradiation spot of $100 \mathrm{~cm}^{2}$. The light of the solar simulator was filtered through an Air Mass 1.5 filter and contains approximately 5\% of UV irradiation at $10 \mathrm{~cm}$ distance. This set of irradiation with monochromatic laser or polychromatic (simulated sunlight) provides information about the photoresponse of the photocatalyst in each spectral region. The amount of hydrogen collected in the head space of the reactor was analysed by injecting $100 \mu \mathrm{L}$ in a gas chromatograph using a MOLSieve column, argon as carrier gas and a TC detector.

\subsection{Synergy of the combination of titanate nanotubes with titania nanoparticles for the photocatalytic hydrogen generation from water methanol mixture using simulated sunlight.}

\subsubsection{Sample preparation.}

The $\mathrm{TiO}_{2}$ precursor used for nanotube production was a commercial $\mathrm{TiO}_{2}$ powder (Millenium PC500, anatase) consisting of pure anatase phase. $\mathrm{TiO}_{2}$ powder (3.6 g) was treated with $90 \mathrm{~mL}$ of aqueous $10 \mathrm{M} \mathrm{NaOH}$ solution in a Teflon-lined autoclave (total volume $200 \mathrm{~mL}$ ) at $125^{\circ} \mathrm{C}$ for 4 days. After the hydrotermal treatment, the solid was collected by filtration and washed by suspending the solid with $2 \mathrm{~L} \mathrm{HCl}$ solution $(\mathrm{pH}=1.3)$ and finally washed with water until neutral $\mathrm{pH}$. The material was dried at $110{ }^{\circ} \mathrm{C}$ during 1 day. The deposition of gold or platinum nanoparticles was carried out by deposition-precipitation method. $0.5 \mathrm{~g}$ of titanate nanotubes were added to an aqueous solution containing the amount of chloroplatinic or chloroauric acid corresponding to $0.5 \mathrm{wt} \%$ of metal loading. The $\mathrm{pH}$ was equilibrated to $\mathrm{pH} 10$ by adding an aqueous solution of sodium hydroxide $0.2 \mathrm{M}$. The solid were washed with MilliQ water and dried in vacuum at ambient temperature. The metal was reduced using a $\mathrm{H}_{2}$ flow of $(100 \mathrm{ml} / \mathrm{min})$ at $300{ }^{\circ} \mathrm{C}$ during $5 \mathrm{~h}$. 
The preparation of mixed materials was carried out mixing an amount of nanotubes $(50 \mathrm{mg}$ ) with the amount of titania needed to reach a composition of $6 \%, 12 \%, 25 \%, 50 \%$ in weight of nanotubes. The powders were suspended in water and the suspensions were sonicated $(150 \mathrm{~W})$ for $30 \mathrm{~min}$ to improve the homogeneity of the suspension and, then, was maintained under magnetic stirring overnight. The suspension was finally filtered and dried at $110{ }^{\circ} \mathrm{C}$.

\subsubsection{Photocatalytic tests for hydrogen production.}

A suspension of the catalyst $\left(25 \mathrm{~mL}, 1{\left.\mathrm{~g} \times \mathrm{L}^{-1}\right)}\right.$ was sonicated for $10 \mathrm{~min}$ and placed in a gas-tight reactor with an irradiation window of $12.56 \mathrm{~cm}^{2}$ provided with temperature and pressure controllers. The reactor was placed in a thermostatic bath with a set point temperature of $25{ }^{\circ} \mathrm{C}$. The suspension was purged with an argon flow of 2 psi for 15 minutes prior to irradiation. The photoreaction was performed from the top of the photoreactor by using a solar simulator (Thermo Oriel $1000 \mathrm{~W}$ ) with an irradiation spot of $100 \mathrm{~cm}^{2}$ placed at distance of $10 \mathrm{~cm}$. The light of the solar simulator was filtered through an Air Mass 1.5 filter and contains approximately $5 \%$ of UV irradiation. The amount of hydrogen collected in the headspace of the reactor was analysed by injecting $100 \mu \mathrm{L}$ in a gas chromatograph using a MOLSieve column, argon as carrier gas and a TC detector.

\subsection{Visible light photocatalytic activity for hydrogen production from water- methanol mixtures of open-framework V-doped mixed-valence titanium phosphate.}

\subsubsection{Material preparation.}

$\left(\mathrm{Ti}^{3+} / \mathrm{Ti}^{4+}\right) \mathrm{P}$ material was prepared as described by Sevov. ${ }^{[2]}$ Titanium metal (Alfa Aesar, 325 mesh) was mixed with $150 \mathrm{~mL}$ of an aqueous solution of 1,3diaminopropane and phosphoric acid (molar proportion Ti 2: 1,3-diaminopropane 2.21: $\mathrm{H}_{3} \mathrm{PO}_{4}$ 13.65: $\mathrm{H}_{2} \mathrm{O}$ 500). The resulting mixture was stirred to achieve a homogeneous suspension and was sealed in a Teflon-lined autoclave. The autoclave was heated in an oven at $170{ }^{\circ} \mathrm{C}$ during a period varying from 1 day (M1), 2 days (M2) and 4 days (M3). After that time the suspension was cooled down to room temperature using an ice bath and finally was filtered and 
exhaustively washed with copious amounts of water, ethanol and acetone. The samples were dried in vacuum at ambient temperature, and were stored under inert atmosphere. The V-doped samples were prepared using the same experimental procedure except for the presence in the starting reaction mixture of an amount of vanadium metal (Alfa-aesar 325 mesh) mixed with titanium metal in weight proportion ranging from 0.25 and $2 \mathrm{wt} \%$. The amount of $\mathrm{V}$ present in the resulting materials was determined by ICP analysis. In this case the autoclave was heated at $170{ }^{\circ} \mathrm{C}$ for 2 days. $\left(\mathrm{Ti}^{3+}\right) \mathrm{TiO}_{2}$ was prepared as reported by Feng. ${ }^{[3]}$ Briefly, $0.3 \mathrm{~g}$ of titanium powder was suspended in $10 \mathrm{~mL}$ of an aqueous solution of $2 \mathrm{M}$ hydrochloric acid. The suspension was stirred to achieve homogeneity and was placed in a Teflon-lined autoclave of $25 \mathrm{~mL}$. The autoclave was placed in an oven at $220^{\circ} \mathrm{C}$ for $12 \mathrm{~h}$. After this time the suspension was cooled to ambient temperature using an ice bath and was finally filtered and washed with water and ethanol.

\subsubsection{Photocatalytic tests for hydrogen production.}

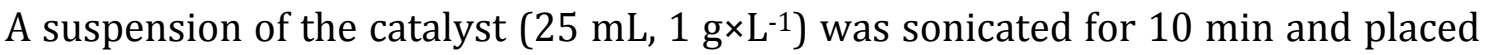
in a closed reactor with an irradiation window of $12.56 \mathrm{~cm}^{2}$ provided with temperature and pressure controllers. The reactor was placed in a thermostatic bath with a set point temperature of $25^{\circ} \mathrm{C}$. The suspension was purged with an argon flow of 2 psi for 15 min prior to irradiation. The photoreaction was performed from the top of the photoreactor by using a visible light LED with an irradiation spot of $4 \mathrm{~cm}^{2}$ placed at a distance of $10 \mathrm{~cm}$. The amount of hydrogen collected in the headspace of the reactor was analysed by injecting $100 \mu \mathrm{L}$ in a gas chromatograph using a MOLSieve column, argon as carrier gas and a TC detector.

\subsubsection{Photocurrent measurements and determination of flat band potential of the conduction band.}

For photocurrent measurements, thin films of $\left(\mathrm{Ti}^{3+} / \mathrm{Ti}^{4+}\right) \mathrm{P}$ were deposited on an FTO electrode ("Solaronix" $0.8 \times 10 \mathrm{~cm}^{2}$ ). Deposition was performed by spreading a paste containing the semiconductor on the FTO surface using the doctor blade procedure. The surface of FTO was defined by adhesive tape. The paste containing the semiconductor was prepared in advance to the deposition of the film onto the electrode by dispersing $100 \mathrm{mg}$ of $\left(\mathrm{Ti}^{3+} / \mathrm{Ti}^{4+}\right) \mathrm{P}, \mathrm{ml}$ of acetone and $1 \mathrm{ml}$ of $\alpha$ - 
terpineol. The suspension was stirred for $24 \mathrm{~h}$ to achieve a homogeneous dispersion. Then, acetone and $\alpha$-terpineol were allowed to evaporate a room temperature to obtain the final dense semiconducting paste. After deposition of the paste, the adhesive tape was removed and the film of semiconductor on FTO was submitted to heating at $80{ }^{\circ} \mathrm{C}$ for $24 \mathrm{~h}$ hour to increases the mechanical resistance of the layer. Photocurrent measurements were carried out using a 150 W Xenon arc lamp as a light source and a PTI model 101 monochromator. Electrical current was measured with an Amel 7050 potentiostat that was connected electrically to the FTO electrode with a platinum wire ("Trallero and Schlee") as counter electrode. The experiments were conducted inside a quartz cuvette $(20 \mathrm{ml})$ that contained an aqueous $0.1 \mathrm{M} \mathrm{KCl}$ solution as electrolyte. The system was purged with nitrogen for at least 15 min before the measurement. Flat conduction band potential was determined by measurements of photocurrent applying increasing bias from 0.3 to $-0.3 \mathrm{Vs}$ (ref. $\mathrm{Ag} / \mathrm{AgCl}$ ) every $0.1 \mathrm{~V}$ (7 points) Photocurrent onset was detected at $335 \mathrm{~nm}$ and the scan rate was of 2 min to stabilize, one of them without irradiation and the next minute with light. The number of charge carriers was determined using the same system connected to a frequency response analyzer. The experiment was carried from $0.5 \mathrm{~V}$ to $-0.5 \mathrm{~V}$ each $0.1 \mathrm{~V}$ (10 points). At each voltage the system scanned in the frequency from $105 \mathrm{~Hz}$ from $0.1 \mathrm{~Hz}$.

\subsubsection{Transient absorption spectroscopy.}

Suspensions of $\left(\mathrm{Ti}^{3+} / \mathrm{Ti}^{4+}\right) \mathrm{P}$ or doped $\left(\mathrm{Ti}^{3+} / \mathrm{Ti}^{4+}\right) \mathrm{P}$ in acetonitrile were prepared by sonicating the powder for $10 \mathrm{~min}$ at $150 \mathrm{~W}$. The amount of solid was set to obtain an optical density at 266 of 0.35 units. The suspensions were persistent during the time needed for the transient absorption spectra measurements (about $3 \mathrm{~h}$ ) without deposition of any solid. $3 \mathrm{ml}$ of these acetonitrile suspensions were placed in a Suprasil quartz cuvette of $1 \times 1 \mathrm{~cm}^{2}$ capped with septa. Suspensions were purged with argon or oxygen (for quenching experiments) for at least $15 \mathrm{~min}$ before the measurements. For ethanol quenching, an argon flow was bubbled trough the suspension of $\left(\mathrm{Ti}^{3+} / \mathrm{Ti}^{4+}\right) \mathrm{P}$ for at least $50 \mathrm{~min}$ before measurements. Transient measurements were carried out using the fourth (266 nm, $20 \mathrm{~mJ} / \mathrm{pulse})$ harmonic of a Nd:YAG laser (7 ns fwhp) as excitation source. The transient signal 
was recorded by the transmission mode using a $150 \mathrm{~W}$ xenon lamp as monitoring beam through fibber optics to collect the transmitted light. The signal from the monochromator/photomultiplier detection system was captured by a Tektronix 2440 digitizer and transferred to a PC computer that controlled the experiment and provided suitable processing and data storage capabilities.

Figure 8.6.1. Current vs. Time plot for sample M2. The bias voltage was scanned from -0.5 to $0.5 \mathrm{~V}$, increasing $0.1 \mathrm{~V}$ every $2 \mathrm{~min}$. The first minute after changing the bias voltage was in the dark and the next minute was illuminated with monochromatic $350 \mathrm{~nm}$ light from a Xenon arc discharge lamp.

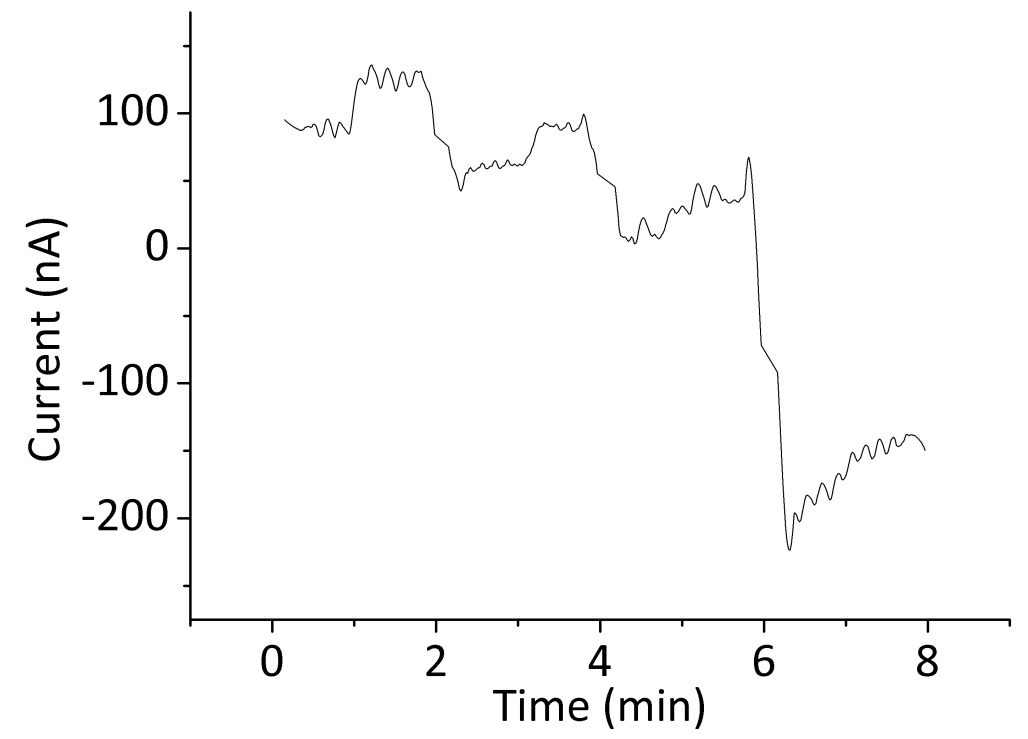


Figure 8.6.2. Current vs. Potential (vs. standard $\mathrm{AgCl} / \mathrm{Ag}$ electrode) curve plotted based on the data points obtained from Figure S4 for M2. The point of zero current corresponds to the flatband potential of the material.

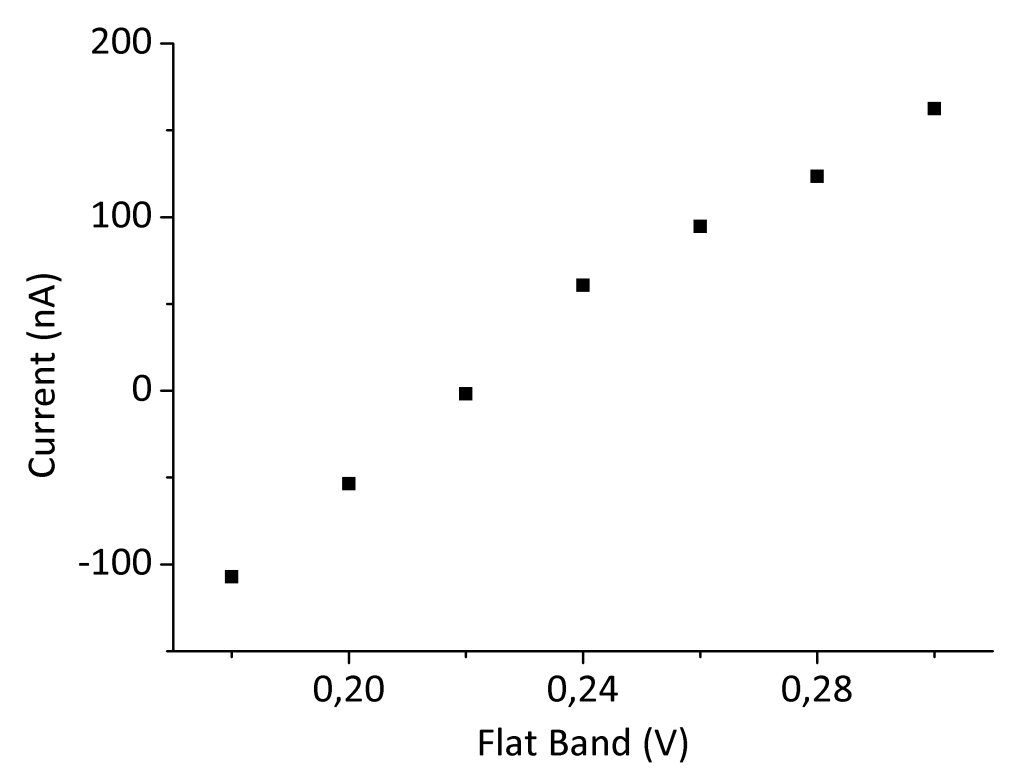

Figure 8.6.3. Nyquist plot obtained for films of M2 supported on FTO electrode.

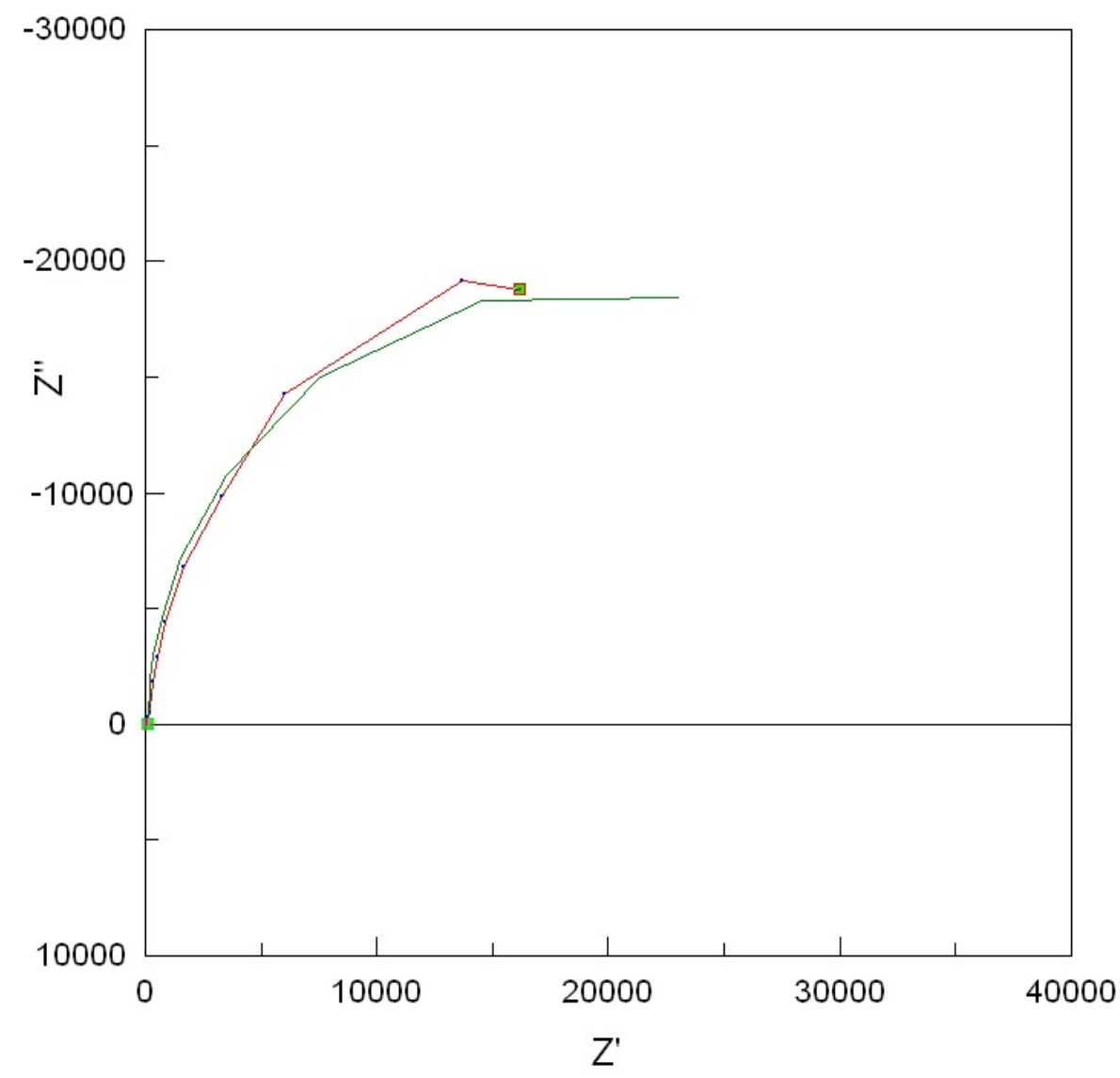




\subsection{Doped framework iron phosphate as novel photocatalyst for hydrogen production from water-methanol mixtures.}

\subsubsection{Materials preparation.}

One-dimensional iron phosphate (FeP) was prepared as reported from Song [4,5]. Briefly, an aqueous solution of ferric chloride, phosphoric acid and ethylendiamine $\left(\mathrm{FeCl}_{3} 0.005 \mathrm{~mol}: \mathrm{H}_{3} \mathrm{PO}_{4} 0.04 \mathrm{~mol}: \mathrm{NH}_{2} \mathrm{CH}_{2} \mathrm{CH}_{2} \mathrm{NH}_{2} 2.5 \mathrm{~mL}: \mathrm{H}_{2} \mathrm{O} 35 \mathrm{~mL}\right)$ was placed into a Teflon-lined autoclave of $200 \mathrm{~mL}$, using a filling factor of $75 \%$.

The autoclave was heated at $150{ }^{\circ} \mathrm{C}$ during $2.5 \mathrm{~h}$ and after this time the reaction mixture was cooled at room temperature using an ice bath. The precipitate was filtered, washed and dried in vacuum at room temperature. The doped materials were prepared following the same synthetic protocol using a mixture of ferric chloride and chromium, manganese or cobalt chloride in proportions ranging from of $0.25-5 \mathrm{wt} \%$ as dopant.

\subsubsection{Photocatalytic tests for hydrogen production.}

A suspension of the catalyst $\left(25 \mathrm{~mL}, 1 \mathrm{~g} \times \mathrm{L}^{-1}\right)$ was sonicated for $10 \mathrm{~min}$ and placed in a closed reactor with an irradiation window of $12.56 \mathrm{~cm}^{2}$ provided with temperature and pressure controllers. The reactor was placed in a thermostatic bath with a set point temperature of $25^{\circ} \mathrm{C}$. The suspension was purged with an argon flow of 2 psi for 15 min prior to irradiation. The photoreaction was performed from the top of the photoreactor by using a visible light LED or a solar simulator (Thermo Oriel $1000 \mathrm{~W}$ ) with an irradiation spot of $100 \mathrm{~cm}^{2}$ placed at a distance of $10 \mathrm{~cm}$. The light of the solar simulator was filtered through an Air Mass 1.5 filter and contains approximately $4 \%$ of UV light. The amount of hydrogen collected in the headspace of the reactor was analysed by injecting $100 \mu \mathrm{L}$ in a gas chromatograph using a MOLSieve column, argon as carrier gas and a TC detector.

\subsubsection{Photocurrent measurements and determination of flat band potential of} the conduction band.

For photocurrent measurements, thin films of FeP were deposited on an FTO electrode ("Solaronix" $0.8 \times 10 \mathrm{~cm}^{2}$ ). Deposition was performed by spreading a paste containing the semiconductor on the FTO surface using the doctor blade 
procedure. The surface of FTO was defined by adhesive tape. The paste containing the semiconductor was prepared in advance to the deposition of the film onto the electrode by dispersing $100 \mathrm{mg}$ of FeP, $1 \mathrm{ml}$ of acetone and $1 \mathrm{ml}$ of $\alpha$-terpineol. The suspension was stirred for $24 \mathrm{~h}$ to achieve a homogeneous dispersion. Then, acetone and $\alpha$-terpineol were allowed to evaporate a room temperature to obtain the final dense semiconducting paste. After deposition of the paste, the adhesive tape was removed and the film of semiconductor on FTO was submitted to heating at $80{ }^{\circ} \mathrm{C}$ for $24 \mathrm{~h}$ hour to increases the mechanical resistance of the layer. Photocurrent measurements were carried out using a $150 \mathrm{~W}$ Xenon arc lamp as a light source and a PTI model 101 monochromator. Electrical current was measured with an Amel 7050 potentiostat that was connected electrically to the FTO electrode with a platinum wire as counter electrode. The experiments were conducted inside a quartz cuvette $(20 \mathrm{ml})$ that contained an aqueous $0.1 \mathrm{M} \mathrm{KCl}$ solution as electrolyte. The system was purged with nitrogen for at least $15 \mathrm{~min}$ before the measurement. Flat conduction band potential was determined by measurements of photocurrent applying increasing bias from 0.3 to -0.3 Vs (ref. $\mathrm{Ag} / \mathrm{AgCl}$ ) every $0.1 \mathrm{~V}$ (7 points). Photocurrent onset was detected at $365 \mathrm{~nm}$ and the scan rate was of 2 min to stabilize, one of them without irradiation and the next min illuminating the cell.

The number of charge carriers was determined using the same system connected to a frequency response analyser. The experiment was carried from $0.5 \mathrm{~V}$ to $-0.1 \mathrm{~V}$ each $0.1 \mathrm{~V}$ (7 points). At each voltage the system scanned in the frequency from 105 $\mathrm{Hz}$ from $0.1 \mathrm{~Hz}$.

\subsubsection{Transient absorption spectroscopy.}

Suspensions of FeP or doped FeP in acetonitrile were prepared by sonicating the powder for $10 \mathrm{~min}$ at $150 \mathrm{~W}$. The amount of solid was set to obtain an optical density at 266 of 0.35 units. The suspensions were persistent during the time needed for the transient absorption spectra measurements (about $3 \mathrm{~h}$ ) without deposition of any solid. $3 \mathrm{ml}$ of these acetonitrile suspensions were placed in a Suprasil quartz cuvette of $1 \times 1 \mathrm{~cm}^{2}$ capped with septa. Suspensions were purged with argon or oxygen (for quenching experiments) for at least $15 \mathrm{~min}$ before the measurements. For ethanol quenching, an argon flow was bubbled through the 
suspension of $\mathrm{FeP}$ for at least $50 \mathrm{~min}$ before measurements. Transient measurements were carried out using the fourth (266 nm, $20 \mathrm{~mJ} /$ pulse) harmonic of a Nd:YAG laser (7 ns fwhp) as excitation source. The transient signal was recorded by transmission mode using a $150 \mathrm{~W}$ xenon lamp as monitoring beam through fiber optics to collect the transmitted light. The signal from the monochromator/photomultiplier detection system was captured by a Tektronix 2440 digitizer and transferred to a PC computer that controlled the experiment and provided suitable processing and data storage capabilities.

\subsection{References.}

[1] A.Naldoni, M. Allieta, S. Santangelo, M. Marelli, F. Fabbri, S. Cappeli, C. L. Bianchi, R. Pisaro, V. Dal Santo, J. Am. Chem. Soc. 2012, 134, 7600-7603.

[2] Ekambaram, S. Sevov, S. C., Angew. Chem., Int. Ed. 1999, 38, 372-375.

[3] Zuo, F. Bozhilov, K. Dillon, R. J. Wang, L. Smith, P. Zhao, X. Bardeen, C. Feng, P., Angew. Chem., Int. Ed. 2012, 51, 6223-6226, S6223/1-S6223/9.

[4] Y. Song, P. Y. Zavalij, N. A. Chernova, M. Suzuki, M. S. Whittingham, Journal of Solid State Chemistry 2003, 175, 63.

[5] Y. Song, P. Y. Zavalij, N. A. Chernova, M. S. Whittingham, Chemistry of Materials 2005, 17, 1139. 
Chapter 9

Conclusions. 


\section{Chapter 10 - Conclusions.}

\subsection{Conclusions.}

In the present Thesis we have described results showing that enhancement of the photocatalytic activity for hydrogen generation from aqueous solutions can be achieved by developing different strategies. Specifically the following conclusions can be deduced from the data presented in the main body of the present study:

1. The presence of platinum in the appropriate proportion can optimize the photocatalytic activity of gold nanoparticle supported on titania for hydrogen production. It was found that depending on the irradiation wavelength and the presence or absence of sacrificial electron donors the influence of platinum is different and in a simplistic way $\mathrm{Au}$ has the role of light harvester in the visible region, while $\mathrm{Pt}$ is better cocatalyst for hydrogen generation. A compromise in the composition of Au-Pt NPs has to be reached depending on the photocatalytic experiment condition.

2. $\mathrm{TiO}_{2}$ nanoparticles obtained by pyrolysis of titanate nanotubes and exhibiting some visible light absorption are more efficient for $\mathrm{H}_{2}$ generation than titanate nanotubes.

3. The concept of heterojunction even with two different titanium semiconductors is also useful for enhancement of the photocatalytic activity of the composite material for photocatalytic hydrogen generation.

4. Mixed $\mathrm{Ti}^{3+} / \mathrm{Ti}^{4+}$ phosphate and iron phosphate behave as semiconductor having conduction band potential and density of carriers of $2 \cdot 10^{20}$ and -0.1 $\mathrm{eV}$ for mixed $\mathrm{Ti}^{3+} / \mathrm{Ti}^{4+}$ phosphate and $2.6 \cdot 10^{20}$ and $0.1 \mathrm{eV}$, respectively for iron phosphate.

5. Both mixed valence $\mathrm{Ti}^{3+} / \mathrm{Ti}^{4+}$ and iron phosphate exhibit photocatalytic activity for hydrogen generation under solar or visible light from water 
methanol mixture. For both phosphates the photocatalytic activity can be optimized by doping.

6. It has been shown for the first time that metal and mixed metal phosphates are semiconductors with considerable flexibility in the composition and with photocatalytic activity for solar fuels production that appears promising at the present and offering various possibilities to be further optimized. 
Appendix 


\subsection{Abstract.}

\section{Abstract.}

The objective of present thesis is to prepare and evaluate photocatalyst for hydrogen generation from water methanol mixture using solar light.

This general objective has been accomplished by applying different methodology in material preparation as well as exploring the photocatalytic activity of novel semiconductors.

In this way after a general introduction to the feed showing the relevance of solar fuels and in particular hydrogen generation, chapter 3 describe a procedure to optimize the nature of the cocatalyst based on noble metals. In this way $\mathrm{Au}-\mathrm{Pt}$ alloy nanoparticles with different composition will be deposited on p25 and they activity correlated with the irradiation wavelength and nature of the alloy.

In chapter 4 and 5 we evaluate the photocatalytic activity of materials derived from titanate nanotubes either by hydrogen annealing ad various temperature (chapter 4) or by forming heterojunction with a combination of titania nanoparticles (chapter 5).

The two final chapter of this thesis report the semiconductor behaviour and the photocatalytic activity of framework phosphate either mixed valence titanium III/IV (chapter 6) or iron (chapter 7) doped with various metal.

The overall results achieved show that is possible to increase the photocatalytic activity of titanium-based materials by apply concept like control of morphology, amorphization of surface of particles, formation of heterojunction and control of the cocatalyst. We have also shows behind oxide, framework phosphate can also be valuable as photocatalysts. 


\section{Resumen.}

El objetivo de la presente tesis doctoral es la preparación de materiales semiconductores y la evaluación de su actividad para la obtención de hidrogeno a partir de mezclas de metanol y agua mediante el empleo de luz solar.

Este objetivo general ha sido logrado aplicando varios métodos basados tanto en el estudio de materiales conocidos como en la preparación de nuevos materiales semiconductores.

De manera que, tras una breve introducción sobre el tema de los combustibles obtenidos a partir del sol como fuente de energía, en el capitulo 3 se presenta un estudio sobre la optimización del sistema cocatalítico constituido por nanopartículas de oro depositadas sobre titania mediante la introducción de un segundo metal noble, como el platino, en forma de aleación. En este estudio se analiza la fotorespuesta del sistema bajo varios tipo de irradiación (ultravioleta o visible) en combinación con la composición de la aleación metálica.

En los capitulo 4 y 5 se evalúa la actividad fotocatalítica de materiales procedentes de nanotubos de titania preparados por calcinación en atmosfera reductora a temperaturas distintas (capitulo 4) o mediante la formación de una heterounión junto a nanopartículas de titanio (capitulo 5).

Los ultimo capítulos de esta tesis tratan del estudio del comportamiento semiconductor de fosfatos de titanio de valencia mixta III/IV (capitulo 6) y de fosfato de hierro (capitulo 7) y de su actividad fotocatalítica para lo obtención de hidrogeno.

Los resultados conseguidos en esta tesis demuestran que es posible mejorar la actividad fotocatalítica de materiales basados en oxido de titanio aplicando estrategias como el control de la morfología, la presencia de dominios amorfos en la superficie de las partículas, la formación de heterouniones y el control del cocatalizador. Además se ha estudiado la posibilidad de emplear fosfatos que también pueden ser empleados como fotocatalizadores. 


\section{Resum.}

L'objectiu de la present tesi doctoral és la preparació de materials semiconductors i l'avaluació de la seua activitat per a l'obtenció d'hidrogen a partir de mescles de metanol i aigua mitjançant l'ocupació de llum solar. Aquest objectiu general ha sigut assolit aplicant diversos mètodes basats tant en l'estudi de materials coneguts com en la preparació de nous materials semiconductors.

De manera que, després d'una breu introducció sobre el tema dels combustibles obtinguts a partir del sol com a font d'energia, en el capítol 3 es presenta un estudi sobre l'optimització del sistema cocatalítico constituït per nanopartícules d'or dipositades sobre titania mitjançant la introducció d'un segon metall noble, com el platí, en forma d'aliatge. En aquest estudi s'analitza la fotoresposta del sistema sota diversos tipus d'irradiació (ultraviolada o visible) en combinació amb la composició de l'aliatge metàl-lic

En els capítol 4 i 5 s'avalua l'activitat fotocatalítica de materials procedents de nanotubos de titania preparats per calcinació en atmosfera reductora a temperatures diferents (capítol 4) o mitjançant la formació d'una heterounió al costat de nanopartícules de titani (capítol 5). Els últims capítols d'aquesta tesi tracten de l'estudi del comportament semiconductor de fosfats de titani de valència mixta III/IV (capítol 6) i de fosfat de ferro (capítol 7) i de la seua activitat fotocatalítica para l'obtenció d'hidrogen.

Els resultats aconseguits en aquesta tesi demostren que és possible millorar l'activitat fotocatalítica de materials basats en oxide de titani aplicant estratègies com el control de la morfologia, la presència de dominis amorfs en la superfície de les partícules, la formació de heterounions i el control del cocatalitzador. A més s'ha estudiat la possibilitat d'emprar fosfats que també poden ser emprats com fotocatalitzadors. 


\subsection{Publications}

The present Thesis gave rise to the next publications:

- Alginate as template in the preparation of active titania photocatalysts. Mireia Buaki-Sogo, Marco Serra, Ana Primo, Mercedes Alvaro and Hermenegildo Garcia.

Chem.Cat.Chem., DOI: 10.1002/cctc.201200386

- Influence of the irradiation wavelength on the photocatalytic activity of AuPt nanoalloys supported on $\mathrm{TiO}_{2}$ for hydrogen generation from water. Pedro Montes-Navajas, Marco Serra and Hermenegildo Garcia.

Catal. Sci. Technol., DOI: 10.1039/C3CY00102D

- Contrasting photocatalytic activity of commercial $\mathrm{TiO}_{2}$ samples for hydrogen generation.

Pedro Montes-Navajas, Marco Serra, Avelino Corma and Hermenegildo Garcia.

Catal. Today, DOI: 10.1016/j.cattod.2013.09.025

- Synergy of the combination of titanate nanotubes with titania nanoparticles for the photocatalytic hydrogen generation from water-methanol mixuture using simulated sunlight.

Marco Serra and Hermenegildo Garcia.

Int. JPhotoenergy, DOI: 10.1155/2014/426797

- Photocatalytic hydrogen generation from water-methanol mixture using "black" anatase obtained by annealing of titanate nanotubes.

Marco Serra, Anish Khan, Abdullah M. Asihi, Samia A. Kosa and Hermenegildo Garcia.

J. Nanopar. Res., Under revision. 
- Visible light photocatalytic activity for hydrogen production from watermethanol mixtures of open-framework $V$-doped mixed-valence titanium phosphate.

Marco Serra, Herme G. Baldovi, Ferran Albarracin and Hermenegildo Garcia. Nano Energy, Under revision.

- Doped framework iron phosphate as novel photocatalyst for hydrogen production from water methanol mixture.

Marco Serra, Herme G. Baldovi, Mercedes Alvaro and Hermenegildo Garcia. Appl. Cat. B, Under revision.

- Influence of the Au particle size on the photocatalytic activity of $\mathrm{Au} / \mathrm{TiO}_{2}$ for hydrogen generation from water.

Marco Serra, Josep Albero Sancho and Hermenegildo Garcia.

Catal. Sci. Technol., Under revision 